\title{
On Differential Invariants of Parabolic Surfaces 1
}

\author{
Zhangchi Chen, Joël Merker,
}

\begin{abstract}
The algebra of differential invariants under $\mathrm{SA}_{3}(\mathbb{R})$ of generic parabolic surfaces $S^{2} \subset \mathbb{R}^{3}$ with nonvanishing Pocchiola $4^{\text {th }}$ invariant $W$ is shown to be generated, through invariant differentiations, by only one other invariant, $M$, of order 5 , having 57 differential monomials. The proof is based on Fels-Olver's recurrence formulas, pulled back to the parabolic jet bundles.
\end{abstract}

[Message to the busy reader: Sections 1 and 2 explain and summarize the contents.]

\section{Introduction}

In continuous and discrete mathematics, group actions are widespread. They also arise in various fields of applied science, especially in classical mechanics [2, 6, 28]. Invariants may be used e.g. to take into account physical symmetries of a body, with the aim of reducing the data size of its analysis.

When available, the associated (algebraic or differential) group invariants enable one to solve equivalence problems, to classify geometric objects, to set up canonical forms for them, to know their symmetries, and to find complete lists of homogeneous models.

This article deals more specifically with differential invariants, group actions being in general nonlinear. It also attempts to handle extended explicit expressions.

Our computational requirements are: be algorithmic, be explicit, and be synthetic what algorithms usually are not! Two main goals are in our minds: compute collections of generating sets of invariants, and understand differential relations among such collections.

We will touch neither rewriting procedures in terms of the generating invariants, nor algorithms for computing inside algebras of invariants. In any case, as soon as the number of independent variables becomes $\geqslant 2$, it is well known that one encounters a high complexity in symbolic expressions. Although objects are inserted in several theoretically satisfactory frameworks, everybody is often left with frustratingly small achievements while playing on any computer.

Most problems in the (infinitely wide) Lie-Cartan theory come up with a given action of a certain $r$-dimensional Lie group $G$ acting on an $m$-dimensional manifold $M$ with $m \geqslant 2$. Attacking these questions often involves studying the induced action of $G$ on submanifolds $S^{p} \subset M^{m}$ of a prescribed dimension $p$, with $1 \leqslant p \leqslant m-1$.

While exploring deeper such problems, the occurrence of certain submanifolds $S^{p} \subset$ $M^{m}$ repeats itself as producing certain (new) sub-submanifolds $S_{2}^{p_{2}} \subset S^{p} \subset M^{m}$, subsub-submanifolds $S_{3}^{p_{3}} \subset S_{2}^{p_{2}} \subset S^{p} \subset M^{m}$, and so on, most of the times after replacing $M^{m}$ with some appropriate, cut-down and branched, jet subspace. Then new (invariant)

${ }^{1} 1$ This work was supported in part by the Polish National Science Centre $(\mathrm{NCN})$ via the grant number 2018/29/B/ST1/0258. 
equations appear, new (invariant) bifurcations are created. So here $S^{p}$ and $M^{m}$ should be thought of as being sub-objects within some of the branches of a certain root problem lying at departure.

Since the dimension $r$ of the group is often (much) higher than the dimension $m$ of the ambient space, in order to somehow 'des-intricate' the group action, one prolongs it to the jet bundles $J_{p, q}^{n}$ of any order $n \geqslant 0$, where $q:=m-p$ is the codimension. Certain general (but complicated) formulas going back to Lie ([22, Ch. 25]) show how tangent directions attached to $S^{p} \subset M^{m}$, and also higher order jets as well, transfer through diffeomorphisms from the base $M$ to the jet bundles $J_{p, q}^{n}$ of any order $n$. Section 3 presents these formulas, and thanks to them, the action of $G$ on $M$ lifts as a $G$-action on every jet space $J_{p, q}^{n}$.

The geometry of submanifolds under Lie transformation groups: their equivalences, their symmetries, their normal forms; is entirely governed by what is known as differential invariants. They are best visualized inside $J_{p, q}^{n}$.

A differential invariant is a (perhaps locally defined) real-valued function $I: J_{p, q}^{n} \longrightarrow \mathbb{R}$ that is invariant under the prolonged group action. Any finite-dimensional Lie group action admits an infinite number of functionally independent differential invariants of progressively higher and higher orders.

A universal question is to find a minimal set of generating differential invariants. Certainly, the minimal number of generating differential invariants cannot be fixed a priori: it strongly depends on the particularities of the group action. Since the $19^{\text {th }}$ Century, the question of finite generation of differential invariants was addressed by several authors, also in the more general context of (infinite-dimensional) Lie pseudo-groups. The fundamental Basis Theorem states that all the differential invariants can be generated, from a finite number of low order invariants, by repeated invariant differentiations.

Serendipitously indeed ([13]), there always exist $p=\operatorname{dim} S$ linearly independent invariant differential operators $\mathscr{D}_{1}, \ldots, \mathscr{D}_{p}$ with the property that each $\mathscr{D}_{j}$ maps every differential invariant $I$ to a differential invariant $\mathscr{D}_{j}(I)$. The great value of such invariant derivations is that one can explicitly write down their action on invariantized jet monomials, and compare the outcome with higher order invariants. This comparison, which incorporates appropriate correction terms, is captured by the celebrated recurrence formulas, set up in the widest context by Fels-Olver in [13], see also Section 14.

Let us repeat that, notwithstanding their power, recent symbolic implementations are often led to unsurmountable obstacles while attempting to explicitly compute invariants, or even cross-sections to the $G$-action on jet spaces. Our Section 11 illustrates this difficulty. But serendipitously again, from the computational side, a minimal amount of data is necessary to set up the key recurrence formulas of Fels-Olver. Remarkably, these formulas can be explicitly determined without knowing the actual formulas for either the differential invariants, or the invariant differential operators, or even the moving frame itself (!).

Indeed, the recurrence formulas can be written with only the knowledge of the infinitesimal generators of the action and the equations of the cross-section. Therefore, understanding these recurrence formulas is the 'master key', according to Olver, that 'unlocks' the structure of the algebra of differential invariants, the determination of generators, and the classification of syzygies. The only required ingredients are the prolongation formulas for the infinitesimal generators, or, equivalently, the Lie matrix, along with the specification of the cross-section normalizations.

Nevertheless, our slogan will be: 
Explicit expressions of invariants are necessary in exploring classification branches.

In differential invariant contexts where no branching is tracked, explicit expressions of invariants are not crucial. In [33], Olver showed that the algebra of differential invariants of a suitably generic hyperbolic or elliptic surface $S \subset \mathbb{R}^{3}$ under the equi-affine group action is generated by a single differential invariant, the third order Pick invariant, with its invariant derivatives. The proof was based on the straight equivariant approach to the method of moving frames. We believe anyway that classification bifurcations also exist for hyperbolic and elliptic surfaces.

Question 1.1. What about parabolic surfaces?

These are (local) surfaces $S^{2} \subset \mathbb{R}^{3}$ of the graphed form $\{u=F(x, y)\}$ whose hessian matrix $\left(\begin{array}{cc}F_{x x} & F_{x y} \\ F_{y x} & F_{y y}\end{array}\right)$ is identically of rank 1, not 2 (elliptic or hyperbolic cases). The vanishing of the Hessian determinant:

$$
0 \equiv F_{x x} F_{y y}-F_{x y}^{2} \quad \text { (at all points }(x, y) \text { ), }
$$

then creates a differential relation which must be differentiated again and again to build up the relevant parabolic jet spaces $P J_{2,1}^{n}$, of any order $n \geqslant 2$. These differential relations also have strong influence on the recurrence formulas. Knowing explicitly the Hessian is unavoidable to study this branch: the category of parabolic surfaces. Other more complicated branching invariants will come up in our deeper explorations, as we will summarize in the next Section 2

The present article therefore opens up a new natural context of jet spaces with differential relations, in which the (cut-down) pulled back recurrence formulas have an entirely invariant meaning. The principle of passing to (bifurcating) submanifolds will be illustrated several times on examples.

It is well known (see Section 3) that the coefficients of the prolonged infinitesimal generators of any group action are polynomial functions of the jet coordinates. In particular, if the action of $G$ is transitive on $M$, which is often the case, and if one normalizes all the order zero coordinates, this implies that the Maurer-Cartan invariants, which appear in the fundamental recurrence formulas, are also rational functions of the collection of generating invariants. As a consequence of the theory, all the higher order differential invariants are rational functions of the generating differential invariants.

Rationality holds true of all the structures studied in this article. The same algebraicity features hold for a large class of pseudo-group actions: the differential invariant algebra is intrinsically rational, in the sense that all recurrence formulas, commutation relations and syzygies, involve rational functions of the basic differential invariants, all of order $\geqslant 1$.

Beyond transitivity of the $G$-action, in Theorem 12.6, we provide a condition on $G$ insuring that all basic differential invariants are of order $\geqslant 2$.

Last but not least, following the approach of Fels-Olver ( $c f$. the recent [36]), we offer in this article another interpretation of Cartan's method of $G$-structure reductions, the application of which goes beyond the plain determination of normal forms for power series given at the origin. One of its advantages is that it includes an explicit approach to finding generating invariants. This method will be presented by elaborating on several examples, before a general theoretical description which will be expressed in a forthcoming publication. 
Acknowledgments. The realization of this research work in differential invariants has received generous financial support from the scientific grant 2018/29/B/ST1/02583 originating from the Polish National Science Center (NCN).

During spring 2019, in May in Orsay, the authors benefited from countless oral exchanges with Paweł Nurowski (Center For Theoretical Physics), who shared his deep knowledge of Cartan's method of equivalence by calculating the explicit numerator of the Pick invariant in full details.

In March 2020 in Orsay, the authors learned a lot from Boris Doubrov (Belarusian State University) on geometric structures of homogeneous real hypersurfaces in complex vector spaces.

Grateful thanks are addressed to an anonymous referee for pointing out the classification of developable surfaces.

\section{Presentation of the Results}

We can now start a precise desciption of our results. Several aspects are true generally, but we restrict ourselves to a presentation of the 2-dimensional case. Most of our considerations will be of local nature. We will assume real analyticity throughout.

We consider the special affine group:

$$
\mathrm{SA}_{3}(\mathbb{R})=\mathrm{SL}_{3}(\mathbb{R}) \ltimes \mathbb{R}^{3},
$$

which consists of invertible linear transformations $(x, y, u) \longmapsto(s, t, v)$ coupled with translations:

$$
\begin{array}{ll}
s=\mathrm{a} x+\mathrm{b} y+\mathrm{c} u+\mathrm{d}, \\
t=\mathrm{k} x+\mathrm{l} y+\mathrm{m} u+\mathrm{n}, \\
v=\mathrm{p} x+\mathrm{q} y+\mathrm{r} u+\mathrm{s},
\end{array} \quad 1=\left|\begin{array}{ccc}
\mathrm{a} & \mathrm{b} & \mathrm{c} \\
\mathrm{k} & \mathrm{I} & \mathrm{m} \\
\mathrm{p} & \mathrm{q} & \mathrm{r}
\end{array}\right|,
$$

preserving volume and orientation. We have:

$$
\operatorname{dim} \mathrm{SA}_{3}(\mathbb{R})=3 \cdot 3-1+3=11 .
$$

We will always consider special affine transformations not far from the identity, hence we may view $\mathrm{SA}_{3}(\mathbb{R})$ as a local Lie group. The full affine group will be denoted $A_{3}(\mathbb{R})=$ $\mathrm{GL}_{3}(\mathbb{R}) \ltimes \mathbb{R}^{3}$.

In the source space $(x, y, u)$, we consider surfaces $S^{2} \subset \mathbb{R}_{x, y}^{2} \times \mathbb{R}_{u}^{1}$ graphed as $\{u=$ $F(x, y)\}$ with convergent power series $F \in \mathbb{R}\{x, y\}$, and similarly, in the target space $(s, t, v)$, we consider graphed analytic surfaces $\{v=G(s, t)\}$ :

$$
\begin{aligned}
& u=\sum_{j=0}^{\infty} \sum_{k=0}^{\infty} F_{j, k} \frac{x^{j}}{j !} \frac{y^{k}}{k !}, \\
& v=\sum_{l=0}^{\infty} \sum_{m=0}^{\infty} G_{l, m} \frac{s^{l}}{l !} \frac{t^{m}}{m !} .
\end{aligned}
$$

Problem 2.2. Determine when two given surfaces $\{u=F(x, y)\}$ and $\{v=G(s, t)\}$ are $\mathrm{SA}_{3}$-equivalent. 
When this holds, by a special affine transformation, every point $(x, y, F(x, y))$ is mapped to a point $(s, t, G(s, t))$, and a fundamental equation holds in $\mathbb{R}\{x, y\}$ :

$$
\mathrm{p} x+\mathrm{q} y+\mathrm{r} F(x, y)+\mathrm{s} \equiv G(\mathrm{a} x+\mathrm{b} y+\mathrm{c} F(x, y)+\mathrm{d}, \quad \mathrm{k} x+\mathrm{l} y+\mathrm{m} F(x, y)+\mathrm{n}) \text {. }
$$

Problem 2.3. Classify surfaces $\{u=F(x, y)\}$ under the $\mathrm{SA}_{3}(\mathbb{R})$ action, especially, find all (locally) homogeneous models.

Problem 2.3 has been studied by means of Lie-theoretical methods. The classification of all $\mathrm{A}_{3}(\mathbb{R})$-homogeneous surfaces with identically vanishing Pick invariant in general affine geometry was obtained in [1].

In [8], all locally homogeneous two-dimensional surfaces in the three-dimensional affine geometry were described, with a list of 18 items. There exist similar classifications for equiaffine geometry [14, 20].

We denote a general element of the special affine group by $g \in \mathrm{SA}_{3}(\mathbb{R})$, and the general transformation as:

$$
s=s(g, x, y, u), \quad t=t(g, x, y, u), \quad v=v(g, x, y, u) .
$$

Definition 2.4. A differential invariant of order $n$ is a function of the horizontal coordinates and the partial derivatives of the graphing function up to order $n$ :

$$
I\left(s, t,\left\{G_{s^{l} t^{m}}(s, t)\right\}_{0 \leqslant l+m \leqslant n}\right) \equiv I\left(x, y,\left\{F_{x^{j} y^{k}}(x, y)\right\}_{0 \leqslant j+k \leqslant n}\right),
$$

which is unchanged after replacement of $(s, t, v)$ in terms of $(g, x, y, u)$, for every $g \in$ $\mathrm{SA}_{3}(\mathbb{R})$.

Problem 2.5. Describe the structure of the algebra of differential invariants of surfaces under the action of $\mathrm{SA}_{3}(\mathbb{R})$.

In this memoir, we will focus on Problems 2.2 and 2.5, in the spirit of [30, 13, 31, 33, 34, 36].

To a graphed surface $\{u=F(x, y)\}$ is associated its Hessian matrix:

$$
\operatorname{Hessian}_{F}=\left(\begin{array}{cc}
F_{x x} & F_{x y} \\
F_{y x} & F_{y y}
\end{array}\right) .
$$

Definition 2.6. [12] A relative invariant is a function satisfying:

$$
P\left(s, t,\left\{G_{s^{l} t^{m}}(s, t)\right\}_{0 \leqslant l+m \leqslant n}\right) \equiv \text { nonzero } \cdot P\left(x, y,\left\{F_{x^{j} y^{k}}(x, y)\right\}_{0 \leqslant j+k \leqslant n}\right),
$$

with a nowhere vanishing factor, at least when $g \in \mathrm{SA}_{3}(\mathbb{R})$ is not far from the identity.

A starting observation (Section 7) is that the Hessian determinant is a relative invariant:

$$
G_{s s} G_{t t}-G_{s t}^{2}=\text { nonzero } \cdot\left(F_{x x} F_{y y}-F_{x y}^{2}\right)
$$

even under general affine transformations. Moreover, Proposition 8.6 shows that the rank of the Hessian matrix remains unchanged through any (special) affine transformation. 
For the general theory of surfaces, this implies an elementary initial branching:

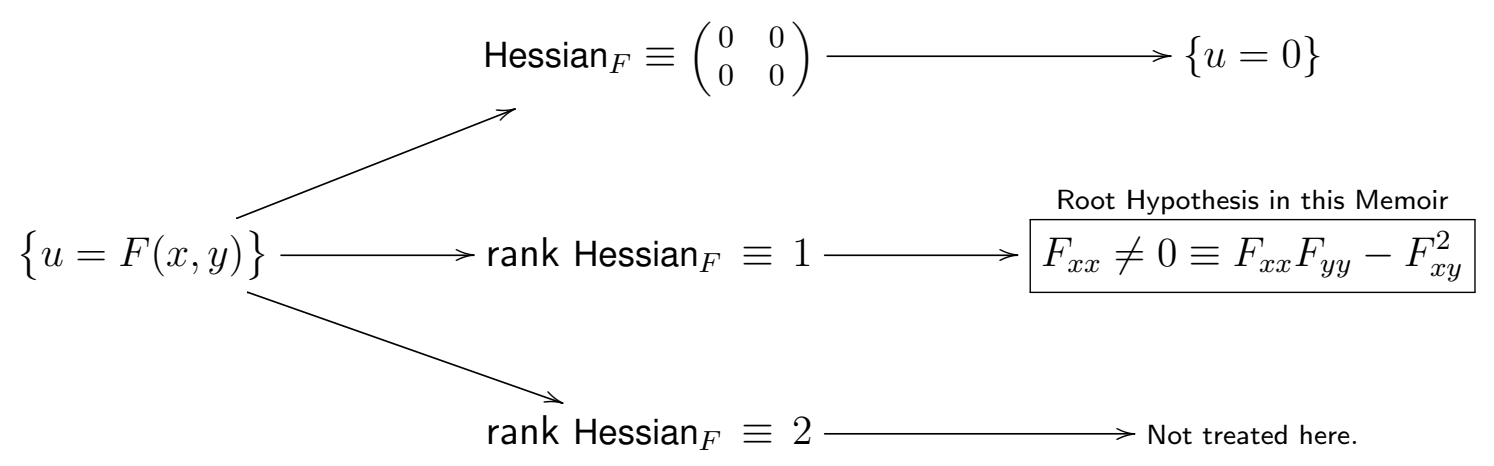

Geometrically, it is clear that the case where the Hessian matrix is identically zero:

$$
0 \equiv F_{x x} \equiv F_{x y} \equiv F_{y y}
$$

is flat in the proper sense, hence there exists a special affine transformation which maps any such $\{u=F(x, y)\}$ to a reference plane $\{v=0\}$. This branch is hence trivial.

The rank 2 case is a wide story in itself, it conducts to the so-called Pick invarian ${ }^{2}$ of order 3, and to further order 4 differential invariants, $c f$. [40, 33].

In this memoir, we will study the middle branch only. After a rotation in the $(x, y)$ space, we can assume that $F_{x x}(x, y) \neq 0$ is nowhere vanishing (our reasonings are local). Then our main root hypothesis will constantly be:

$$
F_{x x} \neq 0 \equiv F_{x x} F_{y y}-F_{x y}^{2} .
$$

Solving:

we may differentiate once:

$$
F_{y y} \equiv \frac{F_{x y}^{2}}{F_{x x}}
$$

$$
\begin{aligned}
& F_{x y y}=2 \frac{F_{x y} F_{x x y}}{F_{x x}}-\frac{F_{x y}^{2} F_{x x x}}{F_{x x}^{2}}, \\
& F_{y y y}=3 \frac{F_{x y}^{2} F_{x x y}}{F_{x x}^{2}}-2 \frac{F_{x y}^{3} F_{x x x}}{F_{x x}^{3}},
\end{aligned}
$$

and so on.

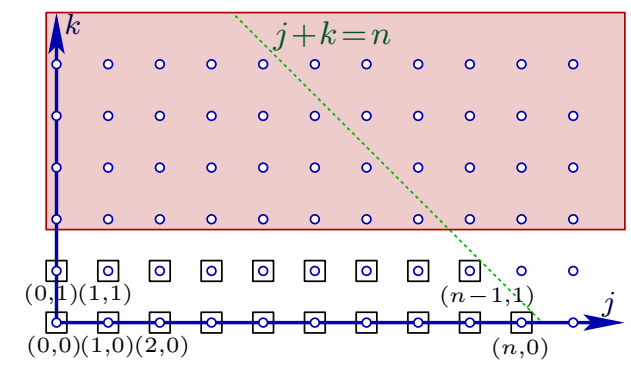

It is easy to convince oneself ( eee Section $\mathbf{1 0}$ ) that every partial derivative $F_{x^{j} y^{k}}$ with $k \geqslant 2$ expresses in terms of the partial derivatives:

$$
\left\{F_{x^{j^{\prime}}}\right\}_{j^{\prime} \leqslant j+k}, \quad\left\{F_{x^{j^{\prime \prime}}}\right\}_{j^{\prime \prime} \leqslant j+k-1} .
$$
help.

${ }^{2}$ On p. 39, the reader will find its explicit expression. Thanks are adressed to Paweł Nurowski for his 
This conducts us to introduce the parabolic jet spaces of any order $n \geqslant 2$ :

$$
P J_{2,1}^{n} \ni \quad\left(x, y, \begin{array}{cccc}
u_{y}, \ldots & \ldots & u_{x^{n-1}}, \\
u, & u_{x}, \ldots, & u_{x^{n-1}}, & u_{x^{n}}
\end{array}\right) \in \mathbb{R}^{3+2 n} .
$$

In effective differential invariant theory, for instance in the case of (not necessarily parabolic) surfaces, under any action of a (local) Lie group $G$, certain relative invariants are encountered, call them:

$$
P=P\left(x, y, u,\left\{u_{x^{j} y^{k}}\right\}_{1 \leqslant j+k \leqslant n}\right), \quad \quad Q, \quad R, \quad \ldots
$$

According to Definition 2.6, their zero-sets $\{\boldsymbol{P}=0\},\{\boldsymbol{Q}=0\}, \ldots$, are invariant under $G$. They are responsible for the creation of branches and of further subbranches:

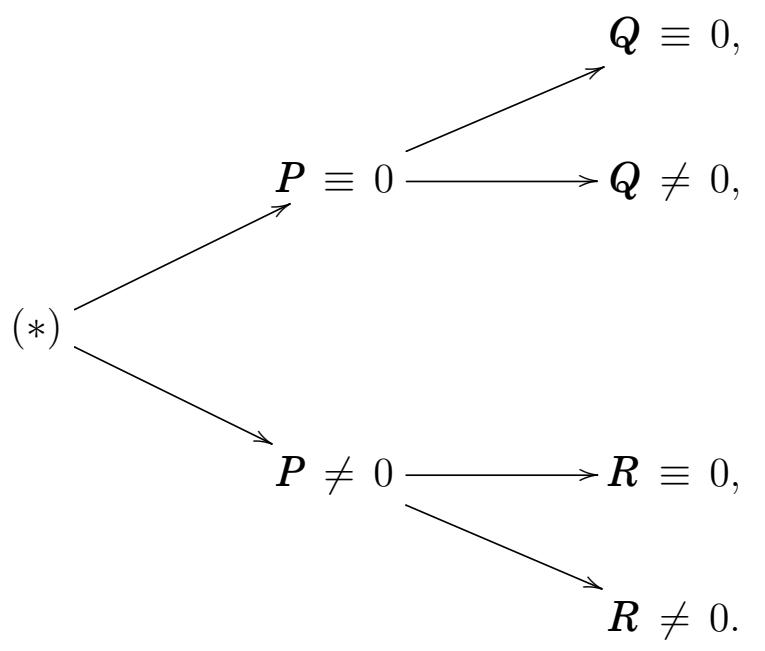

We adopt Lie's principle of thought ([22, Chap. 1]), which admits that either a (relative) differential invariant is identically zero, or it is assumed to be nowhere zero, after restriction to an appropriate open subset. Mixed cases where some (relative) invariant is nonzero on some nonempty open subset and vanishes on a nonempty closed subset are excluded from exploration.

Importantly, as soon as some (relative) invariant vanishes identically, like our Hessian determinant:

$$
H_{F}:=F_{x x} F_{y y}-F_{x y}^{2},
$$

one must express all differential consequences of this assumption in order to explore properly the concerned branch. When, on some (sub)branch, there occurs a simultaneous vanishing of two or more (relative) invariants, one must at first express the differential consequences under a closed workable form, like setting up a meaningful Gröbner basis for the differential ideal generated.

We can now start to present our results. At first, if we abbreviate:

$$
\text { root }:=\begin{aligned}
& 0 \neq F_{x x} \\
& 0 \equiv F_{x x} F_{y y}-F_{x y}^{2}
\end{aligned}=F_{x x} \neq 0 \equiv H_{F}
$$


the branching diagram which summarizes everything is:

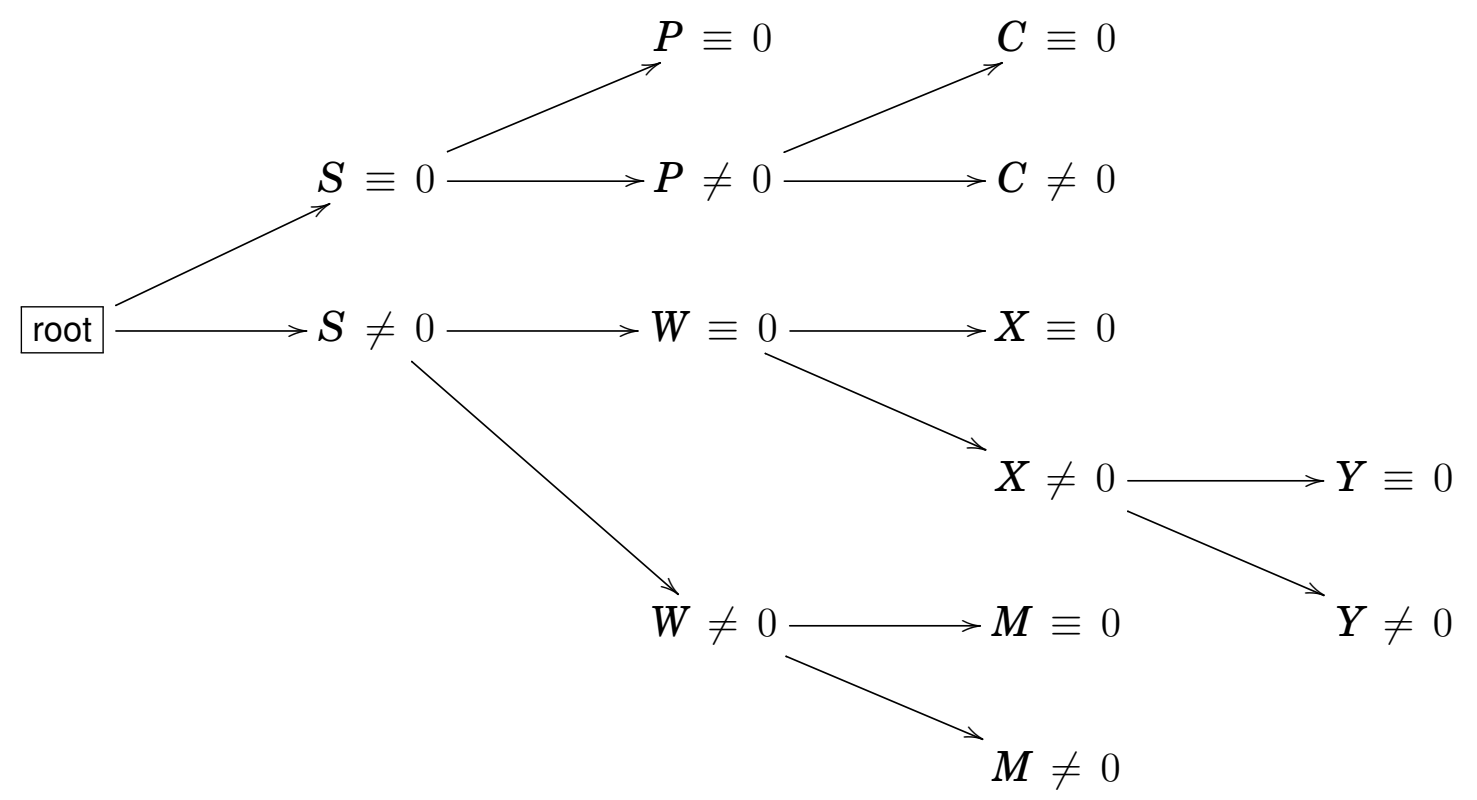

This tree decomposes in 3 main branches, extracted in three diagrams below, just before the statements of 3 associated theorems.

In the first, top branch, $S$ and $P$ are relative invariants:

$$
\begin{aligned}
S & :=\frac{F_{x x} F_{x x y}-F_{x y} F_{x x x}}{F_{x x}^{2}}, \\
P & :=\frac{1}{3} \frac{-5 F_{x x x}^{2}+3 F_{x x} F_{x x x x}}{F_{x x}^{2}},
\end{aligned}
$$

while $C$ is a differential invariant:

$$
C:=\frac{1}{\sqrt{3}} \frac{9 F_{x x}^{2} F_{x x x x x}-45 F_{x x} F_{x x x} F_{x x x x}+40 F_{x x x}^{3}}{\left( \pm 3 F_{x x} F_{x x x x} \mp 5 F_{x x x}^{2}\right)^{3 / 2}} .
$$

In the second, middle branch, $W$ is a differential invariant, but it is assumed to vanish identically, hence it is trivial, and further, $X$ and $Y$ are differential invariants:

$$
\begin{aligned}
& X:=\frac{1}{9} \frac{\left(F_{x x} F_{x x y}-F_{x y} F_{x x x}\right)\left(9 F_{x x}^{2} F_{x x x x x}-45 F_{x x} F_{x x x} F_{x x x x}+40 F_{x x x}^{3}\right)}{F_{x x}^{6}} \\
& Y:=\frac{1}{18} \frac{\left(F_{x x y} F_{x x}-F_{x x x} F_{x y}\right)^{5 / 3}}{F_{x x}{ }^{10}\left(9 F_{x x x x x} F_{x x}{ }^{2}-45 F_{x x x} F_{x x x x} F_{x x}+40 F_{x x x}{ }^{3}\right)}\{ \\
&\left\{11200 F_{x x x}{ }^{8}-12600 F_{x x x}{ }^{3} F_{x x x x x} F_{x x}{ }^{3} F_{x x x x}+13230 F_{x x x} F_{x x x x x} F_{x x}{ }^{4} F_{x x x x}{ }^{2}+1134 F_{x x x} F_{x x x x x} F_{x x}{ }^{5} F_{x x x x x x}\right. \\
&-3150{F_{x x x}}^{2} F_{x x x x} F_{x x}{ }^{4} F_{x x x x x x}-810 F_{x x x x x x x} F_{x x}{ }^{5} F_{x x x} F_{x x x x}-33600 F_{x x x}{ }^{6} F_{x x x x} F_{x x x}-7875 F_{x x x}{ }^{2} F_{x x x x}{ }^{3} F_{x x}{ }^{3} \\
&- 756 F_{x x x}{ }^{2} F_{x x x x x}{ }^{2} F_{x x}{ }^{4}+6720 F_{x x x}{ }^{5} F_{x x x x x} F_{x x}{ }^{2}+31500 F_{x x x}{ }^{4} F_{x x x x}{ }^{2} F_{x x}{ }^{2}-4725 F_{x x x x} F_{x x x}^{4} \\
&- 189 F_{x x x x x x}{ }^{2} F_{x x}{ }^{6}+1890 F_{x x x x}{ }^{2} F_{x x}{ }^{5} F_{x x x x x x}-2835 F_{x x x x} F_{x x}{ }^{5} F_{x x x x x}{ }^{2}+162 F_{x x x x x x x} F_{x x}{ }^{6} F_{x x x x x} \\
&+\left.720 F_{x x x x x x x} F_{x x}{ }^{4} F_{x x x}{ }^{3}\right\} .
\end{aligned}
$$


In the third, last, bottom branch, $W$ is a nontrivial differential invariant:

$$
W:=\frac{F_{x x}^{2} F_{x x x y}-F_{x x} F_{x y} F_{x x x x}+2 F_{x y} F_{x x x}^{2}-2 F_{x x} F_{x x x} F_{x x y}}{\left(F_{x x}\right)^{2}\left(F_{x x} F_{x x y}-F_{x y} F_{x x x}\right)^{2 / 3}},
$$

and $M$ is also a differential invariant:

$$
\begin{aligned}
& M:=\frac{1}{36} \frac{1}{F_{x x}{ }^{6}\left(-F_{x x y} F_{x x}+F_{x x x} F_{x y}\right)\left(F_{x x} F_{x x x x} F_{x y}-F_{x x}{ }^{2} F_{x x x y}+2 F_{x x x} F_{x x y} F_{x x}-2 F_{x x x}{ }^{2} F_{x y}\right)}\{ \\
& \left\{270 F_{x x}{ }^{6} F_{x x x x y} F_{x x y}{ }^{2} F_{x x x x}-72 F_{x x x} F_{x x x x x} F_{x x}{ }^{5} F_{x x y}{ }^{3}+820 F_{x x x} F_{x x}{ }^{3} F_{x x x x}{ }^{3} F_{x y}{ }^{3}-2195 F_{x x x}{ }^{3} F_{x x}{ }^{2} F_{x x x x}{ }^{2} F_{x y}{ }^{3}\right. \\
& +2560 F_{x x x}{ }^{5} F_{x x} F_{x x x x} F_{x y}{ }^{3}+2000 F_{x x x}{ }^{2} F_{x x}{ }^{5} F_{x x x y}{ }^{2} F_{x x y}-2000 F_{x x x}{ }^{3} F_{x x}{ }^{4} F_{x x x y}{ }^{2} F_{x y}-3040 F_{x x x}{ }^{3} F_{x x}{ }^{4} F_{x x x y} F_{x x y}{ }^{2} \\
& -3040 F_{x x x}{ }^{5} F_{x x}{ }^{2} F_{x x x y} F_{x y}{ }^{2}-3840 F_{x x x}{ }^{5} F_{x x y}{ }^{2} F_{x x}{ }^{2} F_{x y}+3840 F_{x x x}{ }^{6} F_{x x y} F_{x x} F_{x y}{ }^{2}-420 F_{x x x x}{ }^{3} F_{x x}{ }^{4} F_{x x y} F_{x y}{ }^{2} \\
& +480 F_{x x x x} F_{x x}{ }^{4} F_{x x y}{ }^{3} F_{x x x}{ }^{2}-420 F_{x x y} F_{x x}{ }^{6} F_{x x x x} F_{x x x y}{ }^{2}+192 F_{x x x}{ }^{4} F_{x x}{ }^{2} F_{x x x x x} F_{x y}{ }^{3}-120 F_{x x x}{ }^{2} F_{x x}{ }^{5} F_{x x x x y} F_{x x y}{ }^{2} \\
& -120 F_{x x x}{ }^{4} F_{x x}{ }^{3} F_{x x x x y} F_{x y}{ }^{2}+36 F_{x x x x x} F_{x x}{ }^{6} F_{x x y}{ }^{2} F_{x x x y}+45 F_{x x}{ }^{6} F_{x x x x y}{ }^{2} F_{x x x} F_{x y}-45 F_{x x}{ }^{5} F_{x x x x x}{ }^{2} F_{x y}{ }^{2} F_{x x y} \\
& +45 F_{x x}{ }^{4} F_{x x x x x}{ }^{2} F_{x y}{ }^{3} F_{x x x}-120 F_{x x}{ }^{4} F_{x x x x x} F_{x y}{ }^{3} F_{x x x x}{ }^{2}-120 F_{x x}{ }^{6} F_{x x x x x} F_{x y} F_{x x x y}{ }^{2}+120 F_{x x}{ }^{5} F_{x x x x y} F_{x x x x}{ }^{2} F_{x y}{ }^{2} \\
& +1280 F_{x x x}{ }^{4} F_{x x y}{ }^{3} F_{x x}{ }^{3}-400 F_{x x x} F_{x x}{ }^{6} F_{x x x y}{ }^{3}-45 F_{x x}{ }^{7} F_{x x x x y}{ }^{2} F_{x x y}-405 F_{x x y}{ }^{3} F_{x x}{ }^{5} F_{x x x x}{ }^{2} \\
& +120 F_{x x}{ }^{7} F_{x x x x y} F_{x x x y}{ }^{2}-1280 F_{x x x}{ }^{7} F_{x y}{ }^{3}-5200 F_{x x x}{ }^{2} F_{x x}{ }^{4} F_{x x x x} F_{x y} F_{x x x y} F_{x x y}+432 F_{x x x} F_{x x}{ }^{4} F_{x x x x} F_{x y}{ }^{2} F_{x x x x x} F_{x x y} \\
& -360 F_{x x x} F_{x x}{ }^{5} F_{x x x x} F_{x y} F_{x x x x y} F_{x x y}+108 F_{x x x} F_{x x}{ }^{5} F_{x x x y} F_{x x x x x} F_{x y} F_{x x y}-2040 F_{x x x} F_{x x}{ }^{4} F_{x x x x}{ }^{2} F_{x y}{ }^{2} F_{x x x y} \\
& +1985 F_{x x x}{ }^{2} F_{x x}{ }^{3} F_{x x x x}{ }^{2} F_{x y}{ }^{2} F_{x x y}+1620 F_{x x x} F_{x x}{ }^{5} F_{x x x x} F_{x y} F_{x x x y}{ }^{2}+4600 F_{x x x}{ }^{3} F_{x x}{ }^{3} F_{x x x x} F_{x y}{ }^{2} F_{x x x y} \\
& +1600 F_{x x x}{ }^{3} F_{x x}{ }^{3} F_{x x x x} F_{x y} F_{x x y}{ }^{2}-4640 F_{x x x}{ }^{4} F_{x x}{ }^{2} F_{x x x x} F_{x y}{ }^{2} F_{x x y}+6080 F_{x x x}{ }^{4} F_{x x}{ }^{3} F_{x x x y} F_{x x y} F_{x y} \\
& +840 F_{x x x x}{ }^{2} F_{x x}{ }^{5} F_{x x y} F_{x y} F_{x x x y}+615 F_{x x x x}{ }^{2} F_{x x}{ }^{4} F_{x x y}{ }^{2} F_{x y} F_{x x x}+600 F_{x x x x} F_{x x}{ }^{5} F_{x x y}{ }^{2} F_{x x x y} F_{x x x} \\
& +336 F_{x x x}{ }^{2} F_{x x}{ }^{4} F_{x x x x x} F_{x y} F_{x x y}{ }^{2}-456 F_{x x x}{ }^{3} F_{x x}{ }^{3} F_{x x x x x} F_{x y}{ }^{2} F_{x x y}-126 F_{x x x}{ }^{2} F_{x x}{ }^{3} F_{x x x x} F_{x y}{ }^{3} F_{x x x x x} \\
& +90 F_{x x x}{ }^{2} F_{x x}{ }^{4} F_{x x x x} F_{x y}{ }^{2} F_{x x x x y}-144 F_{x x x}{ }^{2} F_{x x}{ }^{4} F_{x x x y} F_{x x x x x} F_{x y}{ }^{2}-306 F_{x x}{ }^{5} F_{x x x x x} F_{x y} F_{x x x x} F_{x x y}{ }^{2} \\
& +240 F_{x x x}{ }^{3} F_{x x}{ }^{4} F_{x x x x y} F_{x x y} F_{x y}-180 F_{x x x} F_{x x}{ }^{6} F_{x x x y} F_{x x x x y} F_{x x y}+180 F_{x x x}{ }^{2} F_{x x}{ }^{5} F_{x x x y} F_{x x x x y} F_{x y} \\
& +90 F_{x x}{ }^{6} F_{x x x x x} F_{x y} F_{x x x x y} F_{x x y}-90 F_{x x}{ }^{5} F_{x x x x x} F_{x y}{ }^{2} F_{x x x x y} F_{x x x}+240 F_{x x}{ }^{5} F_{x x x x x} F_{x y}{ }^{2} F_{x x x x} F_{x x x y} \\
& \left.-240 F_{x x}{ }^{6} F_{x x x y y} F_{x x x x} F_{x y} F_{x x x y}\right\} \text {. }
\end{aligned}
$$

It is important to show these invariants, because they not only cause the branchings, but also, they will constitute generating collections for the full algebras of differential invariants.

We may now state our results for the three kinds of branches. We always start from our root assumption.

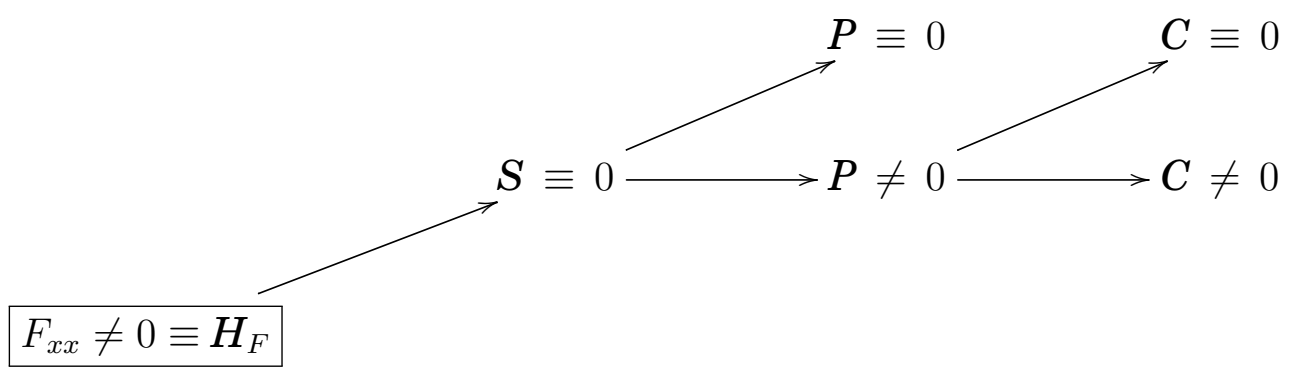

The full affine group in two dimensions is $A_{2}(\mathbb{R})=G_{2}(\mathbb{R}) \ltimes \mathbb{R}^{2}$.

Theorem 2.7. Within the branch $S \equiv 0$ : 
(1) Every surface $S^{2} \subset \mathbb{R}^{3}$ is special affinely equivalent to the product of a curve in $\mathbb{R}_{x, u}^{1+1}$ and $\mathbb{R}_{y}^{1}$, and $\mathrm{SA}_{3}(\mathbb{R})$-equivalences amount to $\mathrm{A}_{2}(\mathbb{R})$-equivalences of such curves;

(2) There is a relative invariant $P$ of order 4 ;

(3) When $P \equiv 0$, the surface is $\mathrm{SA}_{3}$-equivalent to $\left\{u=x^{2}\right\}$, the product of a parabola and $\mathbb{R}_{y}^{1}$, and conversely;

(4) When $P \neq 0$, the surface is, in a unique way, $\mathrm{SA}_{3}$-equivalent to:

$$
u=\frac{x^{2}}{2 !} \pm \frac{x^{4}}{4 !}+F_{5,0} \frac{x^{5}}{5 !}+\sum_{j \geqslant 6} F_{j, 0} \frac{x^{j}}{j !},
$$

and the collection of coefficients $F_{5,0},\left\{F_{j, 0}\right\}_{j \geqslant 6}$ is in one-to-one correspondence with equivalent classes.

Here:

$$
F_{5,0}=F_{x x x x x}(0)=\text { value of } C \text { at the origin. }
$$

Infinitely many differential invariants correspond to these coefficients $F_{j, 0}$, as we will soon explain.

Question 2.8. How to compute explicitly differential invariants?

It is clear that $\mathrm{SA}_{3}(\mathbb{R})$ contains all translations:

$$
s=x+\mathrm{d}, \quad t=y+\mathrm{n}, \quad v=u+\mathrm{s} .
$$

This implies - exercise from Definition 2.4, or see Theorem 12.3 - that every differential invariant:

$$
I(\underbrace{x, y, u}_{\text {absent }},\left\{u_{x^{j} y^{k}}\right\}_{1 \leqslant j+k \leqslant n}),
$$

must depend only on jet derivatives of order $\geqslant 1$.

To compute these invariants $I$, we start from a power series at the origin:

$$
u=\sum_{j+k \geqslant 1} F_{j, k} \frac{x^{j}}{j !} \frac{y^{k}}{k !},
$$

and we progressively perform (several) 'simple', 'natural', special affine transformations in order to annihilate/normalize as much as possible Taylor coefficients $F_{j, k}$. Rigorous descriptions illustrated by examples will be provided in Sections 13, 16, 17, 18, 19, but here, we only present general ideas. One main feature of the process is its progressivity.

At the end, we reach a certain 'normal form':

$$
v=\sum_{l+m \geqslant 1} G_{l, m} \frac{s^{l}}{l !} \frac{t^{m}}{m !},
$$

in which several coefficients $G_{l, m}$ are 'simplified', for instance as in Theorem 2.7 above:

$$
G_{1,0}=G_{0,1}=0, \quad G_{2,0}=1, \quad G_{3,0}=0, \quad \text { etc. }
$$

Certainly, the full composition of all the progressively normalizing maps belongs to $\mathrm{SA}_{3}(\mathbb{R})$, hence is of the form $(2.1)$ for some specific constants a, .., s. These constants are complicated at the end, but step-by-step they are simple, only the full composition of normalizing maps creates complexity.

After the process is pushed at its farthest point, the identity map of $S_{3}(\mathbb{R})$ is the only transformation which leaves untouched the 'normal form' of the power series $v=G(s, t)$. 
While normalizing low order Taylor coefficients, we also keep track (on a computer) of the way how the other (higher order) Taylor coefficients are modified. At the end, we receive formulas:

$$
G_{l, m}=\Pi_{l, m}\left(\left\{F_{j, k}\right\}_{1 \leqslant j+k \leqslant l+m}\right) \quad(l+m \geqslant 1) .
$$

Then granted that:

$$
F_{j, k}=u_{x^{j} y^{k}}(0,0),
$$

all the desired genuine differential invariants are obtained simply by replacing in these formulas Taylor coefficients by jet coordinates:

$$
I_{l, m}:=\Pi_{l, m}\left(\left\{u_{x^{j} y^{k}}(x, y)\right\}_{1 \leqslant j+k \leqslant l+m}\right) \quad(l+m \geqslant 1) .
$$

Importantly, the hypothesis that the group contains all translations guarantees (Theorem 12.3 that we obtain the expressions of all differential invariants at every point $(x, y)$ near the origin.

This process could be explained abstractly in any dimension (forthcoming). During normalizations, relative invariants play a crucial role.

Observation 2.9. Any (relative) invariant $P$ :

- Either creates a new branch $P \equiv 0$ to be explored farther;

- or is absorbed, when $P \neq 0$, into some constant by normalization.

This is, for instance, true of $S, P, W, X$ : when they are nonzero, they will be used to normalize some Taylor coefficients.

Theorem 2.10. With the assumption $S \neq 0$, there is exactly one differential invariant of fourth order, $W$.

We can now state our second result for the second, middle branch. (The third, bottom branch will also assume $S \neq 0$.)

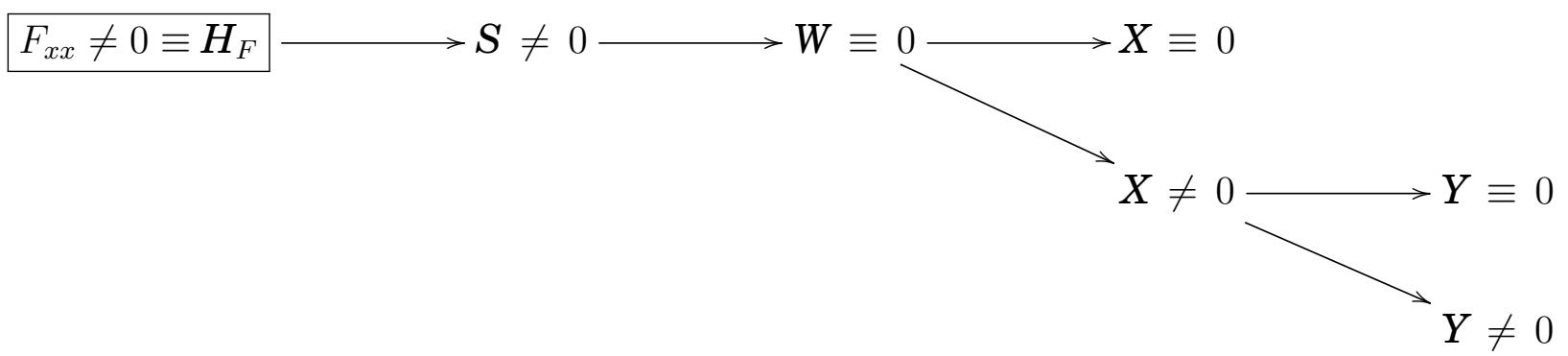

Theorem 2.11. Within the branch $S \neq 0, W \equiv 0$ :

(1) There is a single invariant, $X$, of order 5;

(2) When $X \equiv 0$, every surface $S^{2} \subset \mathbb{R}^{3}$ is $\mathrm{SA}_{3}$-equivalent to the model:

$$
\begin{aligned}
u & =\frac{1}{2} \frac{x^{2}}{1-y} \\
& =\frac{x^{2}}{2}+\frac{x^{2} y}{2}+\frac{x^{2} y^{2}}{2}+\frac{x^{2} y^{3}}{2}+\cdots+\frac{x^{2} y^{k}}{2}+\cdots ;
\end{aligned}
$$


(3) When $X \neq 0$, every surface is $\mathrm{SA}_{3}$-equivalent to:

$$
\begin{aligned}
u=\frac{x^{2}}{2}+\frac{x^{2} y}{2}+\frac{x^{2} y^{2}}{2}+F_{5,0} \frac{x^{5}}{120}+\frac{x^{2} y^{3}}{2}+4 F_{5,0} \frac{x^{5} y}{120}+\frac{x^{2} y^{4}}{2}+F_{7,0} \frac{x^{7}}{5040} & +20 F_{5,0} \frac{x^{5} y^{2}}{240}+\frac{x^{2} y^{5}}{2}+ \\
& +\sum_{j+k \geqslant 8} F_{j, k} x^{j} y^{k}
\end{aligned}
$$

with:

$$
\begin{aligned}
& F_{5,0}=\text { value of } X \text { at the origin, } \\
& F_{7,0}=\text { value of } Y \text { at the origin; }
\end{aligned}
$$

(4) The collection of coefficients $F_{5,0}, F_{7,0},\left\{F_{j, 0}\right\}_{j \geqslant 8}$ is in one-to-one correspondence with equivalent classes.

Lastly, we treat the main (thickest) branch:

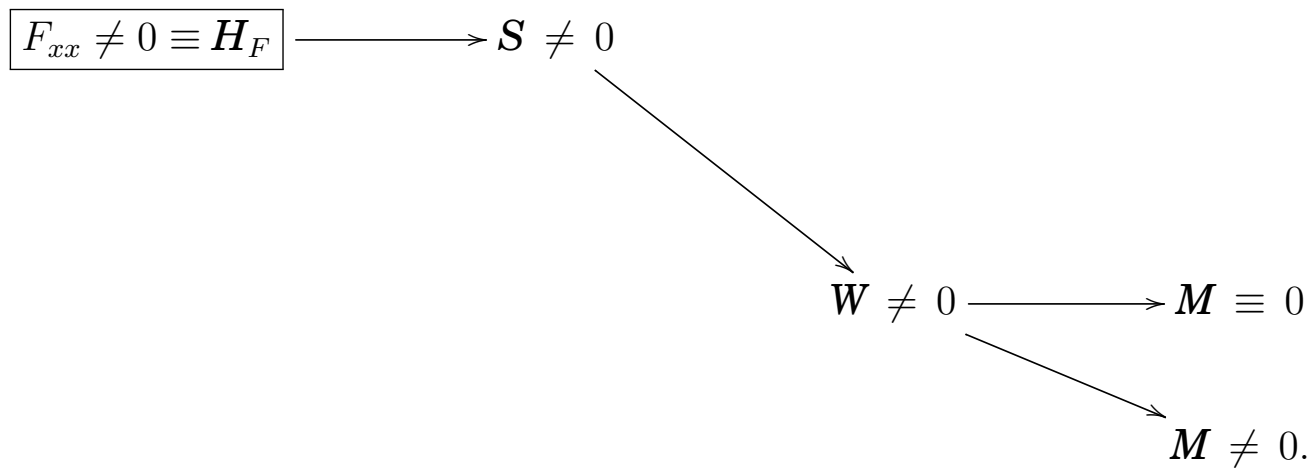

Theorem 2.12. Within the main branch $S \neq 0, W \neq 0$ :

(1) There is a single differential invariant $M$, of order 5 , differentiably independent of $W$;

(2) Every surface $S^{2} \subset \mathbb{R}^{3}$ is $\mathrm{SA}_{3}$-equivalent to:

$$
\begin{aligned}
u=\frac{x^{2}}{2}+\frac{x^{2} y}{2}+F_{3,1} \frac{x^{3} y}{6}+\frac{x^{2} y^{2}}{2}+F_{5,0} \frac{x^{5}}{120}+6 F_{3,1} \frac{x^{3} y^{2}}{12} & +\frac{x^{2} y^{3}}{2}+ \\
& +\sum_{j+k \geqslant 6} F_{j, k} x^{j} y^{k},
\end{aligned}
$$

with:

$$
\begin{aligned}
& F_{3,1}=\text { value of } W \text { at the origin, } \\
& F_{5,0}=\text { value of } M \text { at the origin; }
\end{aligned}
$$

(3) Any other surface $\{v=G(s, t)\}$ within the same branch similarly put into the form:

$$
\begin{aligned}
v=\frac{s^{2}}{2}+\frac{s^{2} t}{2}+G_{3,1} \frac{s^{3} t}{6}+\frac{s^{2} t^{2}}{2}+G_{5,0} \frac{s^{5}}{120}+6 G_{3,1} \frac{s^{3} t^{2}}{12} & +\frac{s^{2} t^{3}}{2}+ \\
& +\sum_{l+m \geqslant 6} G_{l, m} s^{l} t^{m},
\end{aligned}
$$

is $\mathrm{SA}_{3}$-equivalent to $\{u=F(x, y)\}$ above if and only if all (independent) Taylor coefficients in the parabolic jet space match:

$$
\begin{aligned}
& G_{3,1}=F_{3,1}, \quad G_{5,0}=F_{5,0}, \quad \begin{array}{ll}
G_{l, 0}=F_{l, 0} & (l \geqslant 6), \\
& G_{l, 1}=F_{l, 1}
\end{array} \quad(l \geqslant 5) .
\end{aligned}
$$


In these three Theorems 2.7, 2.11, 2.12, there always exist, according to Fels-Olver [13], two invariant differential operators $\mathscr{D}_{1}$ and $\mathscr{D}_{2}$ satisfying:

$$
\mathscr{D}_{i}(\text { differential invariant })=\text { differential invariant } \quad(i=1,2),
$$

and they are non-commuting, in general. Invariantly derivating an invariant means applying $\mathscr{D}_{1}$ and $\mathscr{D}_{2}$ several times, in any order. More explanations will be given in Sections 4, 5, 14, see especially (14.2).

Theorem 2.13. The full algebra of differential invariants under the action of $\mathrm{SA}_{3}(\mathbb{R})$ is generated by:

(1) In the branch $S \equiv 0, P \neq 0$ :

\section{$C$ and its invariant derivatives;}

(2) In the branch $S \neq 0, W \equiv 0$ :

\section{$X, Y$ and their invariant derivatives;}

(3) In the branch $S \neq 0, W \neq 0$ :

\section{$W, M$ and their invariant derivatives.}

It is well known that parabolic surfaces coincide with developable surfaces. While normalizing the $\mathrm{SA}_{3}(\mathbb{R})$-moving frames of parabolic surfaces using Cartan's formalism, Guggenheimer obtained degenerate branches of cylinders and cones in [14, p. 295]. His work can be re-expressed in terms of explicit differential invariants. In Section 22, we will prove that a parabolic surface is a cylinder if and only if $S \equiv 0$; a cone if and only if $S \neq 0$ and $W \equiv 0$; a tangential surface (tangents of a space curve) if and only if $S \neq 0$ and $W \neq 0$.

\section{Graph Transformations and Jet Spaces}

We provide here a reminder on how graphs and their transformations can be lifted to jet spaces of any order. All functions and geometric objects will be assumed analytic, or smooth, or even of a finite but high enough differentiability class, provided all differentiations necessary in our processes are permitted. Over either $\mathbb{R}$ or $\mathbb{C}$, we will mostly work in a local framework, not mentioning restricted open (sub)sets in which calculations are true. We will deal only with finite-dimensional Lie groups (but see [37, 38]).

3.1. Jet spaces and jet notations. Consider $p \geqslant 1$ independent variables $\left(x^{1}, \ldots, x^{p}\right)$ and also $q \geqslant 1$ variables $\left(u^{1}, \ldots, u^{q}\right)$ which are dependent in the sense that they should be components $u^{\alpha}=\mathrm{u}^{\alpha}(x), 1 \leqslant \alpha \leqslant q$, of maps $\mathrm{u}: \mathbb{R}^{p} \longrightarrow \mathbb{R}^{q}$. For any $n \geqslant 0$, introduce the $n^{\text {th }}$ order jet space of such maps:

$$
J_{x, u}^{n}:=J^{n}\left(\mathbb{R}_{x}^{p} \longrightarrow \mathbb{R}_{u}^{q}\right) \equiv J_{p, q}^{n} .
$$

This $J_{x, u}^{n}$ is equipped with coordinates:

$$
z^{(n)}:=\left(x^{j}, u^{\alpha}, u_{x^{J}}^{\beta}\right)
$$

where $J=\left(j_{1}, \ldots, j_{\lambda}\right)$ is an unordered multi-index, with $1 \leqslant \lambda=|J| \leqslant n$, with $1 \leqslant$ $j_{1}, \ldots, j_{\lambda} \leqslant p$, and where each $u_{x^{J}}^{\alpha} \equiv u_{J}^{\alpha}$ is an independent real coordinate corresponding to the partial derivative:

$$
\frac{\partial u^{\alpha}(x)}{\partial x^{j_{1}} \ldots \partial x^{j_{\lambda}}} \longleftrightarrow u_{x^{J}}^{\alpha} \equiv u_{J}^{\alpha} \quad \quad\left(1 \leqslant \alpha \leqslant q, J=\left(j_{1}, \ldots, j_{\lambda}\right)\right)
$$


For instance when $p=q=1$, we will denote jet coordinates sometimes $u_{x}, u_{x x}, u_{x x x}$, $\ldots$, sometimes $u_{1}, u_{2}, u_{3}, \ldots$ Thus:

$$
\operatorname{dim} J_{p, q}^{n}=p+q\left(\begin{array}{c}
p+n \\
n
\end{array}\right) .
$$

When $p \geqslant 2$, say with $p=2$ and $q=1$ to simplify, an alternative multi-index notation will sometimes be employed, especially when working on any current computer algebra system like MATHEMATICA, MAPLE, SAGE and others, for instance:

$$
u_{x^{1}} \equiv u_{1} \equiv u_{[1,0]}, \quad u_{x^{2}} \equiv u_{2} \equiv u_{[0,1]}, \quad u_{x^{1} x^{2} x^{1}} \equiv u_{1,2,1} \equiv u_{[2,1]} .
$$

To translate, we just count the number of times every index $1 \leqslant i \leqslant p$ appears in the sequence $j_{1}, \ldots, j_{\lambda}$ :

$$
\kappa_{i}:=\operatorname{Card}\left\{1 \leqslant \nu \leqslant \lambda: j_{\nu}=i\right\},
$$

and we admit the notational coincidences:

$$
u_{x^{j_{1} \ldots x^{j_{\lambda}}}} \equiv u_{j_{1}, \ldots, j_{\lambda}} \equiv u_{\left[\kappa_{1}, \ldots, \kappa_{p}\right]} \cdot
$$

Sometimes even, when employing the second multi-index notation, we will drop the brackets:

$$
u_{\left[\kappa_{1}, \ldots, \kappa_{p}\right]} \equiv u_{\kappa_{1}, \ldots, \kappa_{p}},
$$

especially when studying parabolic surfaces $S^{2} \subset \mathbb{R}^{3}$ later on. There will be no risk of confusion.

3.2. Prolongations of diffeomorphisms to jet spaces. Without full proofs but with presentations of ideas, let us review the fundamental prolongation formulas which are anyway rarely used in applications because of their complexity. For completeness of the exposition, it is nevertheless necessary to be a bit specific about that, since in some cases anyway, symbolic computer softwares happen to be able to handle such formulas.

Consider a diffeomorphism $\phi: \mathbb{R}^{p+q} \longrightarrow \mathbb{R}^{p+q}$ between equidimensional spaces:

$$
(x, u) \longmapsto(X(x, u), U(x, u))=(X, U),
$$

the target space $\mathbb{R}_{X, U}^{p+q}$ being equipped with similar coordinates $X \in \mathbb{R}^{p}$ and $U \in \mathbb{R}^{q}$. In later paragraphs, we will employ the notation $(y, v)$ instead of $(X, U)$ - clearer here as it indicates parallels and formal analogies.

We must also introduce functional symbols for the first $p$ components and the last $q$ components of such a diffeomorphism:

$$
X^{i}=\varphi^{i}(x, u), \quad U^{\alpha}=\psi^{\alpha}(x, u) \quad(1 \leqslant i \leqslant p, 1 \leqslant \alpha \leqslant q) .
$$

But at the end, we will come back to the more parallel notation:

$$
X^{i}=X^{i}(x, u), \quad U^{\alpha}=U^{\alpha}(x, u) \quad(1 \leqslant i \leqslant p, 1 \leqslant \alpha \leqslant q) .
$$

The splitting $\mathbb{R}_{x}^{p} \times \mathbb{R}_{u}^{q}$ is motivated by the fact that we are interested in submanifolds $\{u=f(x)\}$ that are graphs of maps $\mathbb{R}^{p} \ni x \longmapsto f(x) \in \mathbb{R}^{q}$. Similar graphs $\{U=F(X)\}$ exist in the target space $\mathbb{R}_{X}^{p} \times \mathbb{R}_{U}^{q}$. We are mainly interested in how source graphs $\{u=$ $f(x)\}$ are transformed into target graphs $\{U=F(X)\}$, provided our diffeomorphism:

$$
(x, u) \longmapsto(\varphi(x, u), \psi(x, u))
$$

is not too far from the identity map, and especially, does not "rotate" too much tangent directions to our graph $\{u=f(x)\}$. 
More precisely, we assume that on restriction to the graph $\{u=f(x)\}$, the first $p$ coordinates $\varphi^{1}, \ldots, \varphi^{p}$ or the diffeomorphism when equated to $p$ target independent horizontal coordinates $X^{1}, \ldots, X^{p}$ :

$$
X=\varphi(x, f(x)) \longleftrightarrow \Upsilon(X)=x
$$

can be solved by means of the implicit function theorem. Equivalently, we are assuming that the Jacobian determinant of the map $x \longmapsto \varphi(x, f(x))$ between equidimensional $\mathbb{R}^{p} \longrightarrow \mathbb{R}^{p}$ is nowhere vanishing:

$$
\begin{aligned}
0 & \neq \operatorname{det}\left(\frac{\partial}{\partial x^{i}}\left[\varphi^{j}(x, f(x))\right]\right)_{1 \leqslant j \leqslant p \text { rows }}^{1 \leqslant i \leqslant p \text { columns }} \\
& =\operatorname{det}\left(\frac{\partial \varphi^{j}}{\partial x^{i}}+\sum_{1 \leqslant \alpha \leqslant q} \frac{\partial f^{\alpha}}{\partial x^{i}} \frac{\partial \varphi^{j}}{\partial u^{\alpha}}\right)_{1 \leqslant j \leqslant p}^{1 \leqslant i},
\end{aligned}
$$

so that the implicit function theorem really applies to the $p$ equations $X^{j}=\varphi^{j}(x, f(x))$ to solve $x=\left(x^{1}, \ldots, x^{p}\right)$ in terms of $X=\left(X^{1}, \ldots, X^{p}\right)$ by means of a certain map $\Upsilon: \mathbb{R}^{p} \longrightarrow \mathbb{R}^{p}$ as written above:

$$
x^{i}=\Upsilon^{i}\left(X^{1}, \ldots, X^{p}\right)
$$

By definition, the map $X \longmapsto \Upsilon(X)$ satisfies $p$ identities:

$$
X^{j} \equiv \varphi^{j}(\Upsilon(X), f(\Upsilon(X)))
$$

which can be differentiated with respect to all the $X^{\ell}$ for $1 \leqslant \ell \leqslant p$ :

$$
\begin{aligned}
\delta_{\ell}^{j} & =\sum_{i=1}^{p} \frac{\partial \varphi^{j}}{\partial x^{i}} \frac{\partial \Upsilon^{i}}{\partial X^{\ell}}+\sum_{\alpha=1}^{q} \frac{\partial \varphi^{j}}{\partial u^{\alpha}} \sum_{i=1}^{p} \frac{\partial f^{\alpha}}{\partial x^{i}} \frac{\partial \Upsilon^{i}}{\partial X^{\ell}} \\
& =\sum_{i=1}^{p}(\underbrace{\frac{\partial \varphi^{j}}{\partial x^{i}}+\sum_{1 \leqslant \alpha \leqslant q} \frac{\partial f^{\alpha}}{\partial x^{i}} \frac{\partial \varphi^{j}}{\partial u^{\alpha}}}_{\text {recognize Jacobian determinant }}) \frac{\partial \Upsilon^{i}}{\partial X^{\ell}}
\end{aligned}
$$

and since the determinant is assumed nonzero, this linear system can be inverted in order to determine the partial derivatives $\frac{\partial \Upsilon^{i}}{\partial X^{\ell}}$.

Before doing this, in order to rewrite this system in a more compact and conceptual form, let us introduce the $p$ total differentiation operators:

$$
\mathrm{D}_{x^{i}}:=\frac{\partial}{\partial x^{i}}+\sum_{\alpha=1}^{q} u_{i}^{\alpha} \frac{\partial}{\partial u^{\alpha}}+\sum_{\# J \geqslant 1} \sum_{\alpha=1}^{q} u_{J, i}^{\alpha} \frac{\partial}{\partial u_{J}^{\alpha}}
$$

$(1 \leqslant i \leqslant p)$

These $\mathrm{D}_{x^{i}}$ are here written as infinite formal operators, but anyway, they will always act in a finite truncated way on every function encountered, for instance:

$$
\mathrm{D}_{x^{i}}\left(\varphi^{j}\right)=\left(\frac{\partial}{\partial x^{i}}+\sum_{\alpha=1}^{q} u_{i}^{\alpha} \frac{\partial}{\partial u^{\alpha}}+0\right)\left(\varphi^{j}(x, u)\right) .
$$

On restriction (pullback) to a graph $(x, f(x))$, the independent jet variables $u_{i}^{\alpha} \longleftrightarrow$ $\frac{\partial f^{\alpha}}{\partial x^{i}}(x)$ become of course partial derivatives of the graphing functions $f^{\alpha}\left(x^{1}, \ldots, x^{n}\right)$, and 
therefore the above linear system rewrites compactly as:

$$
\delta_{\ell}^{j}=\sum_{i=1}^{p} \mathrm{D}_{x^{i}}\left(\varphi^{j}\right) \frac{\partial \Upsilon^{i}}{\partial X^{\ell}}
$$

For instance, in the case where $p=1$ :

$$
1=\mathrm{D}_{x}(\varphi) \Upsilon_{X}
$$

we can solve:

$$
\Upsilon_{X}=\frac{1}{\mathrm{D}_{x}(\varphi)},
$$

while an unpleasant matrix inversion will be required as soon as $p \geqslant 2$.

Next, in the simplest case where $p=1=q$, the graph $(x, f(x))$ is transformed into the target graph:

$$
\begin{aligned}
U & =\psi(x, f(x)) \\
& =\psi(\Upsilon(X), f(\Upsilon(X))) \\
& =: F(X),
\end{aligned}
$$

whose tangent directions are obtained by plain derivation:

$$
\begin{aligned}
F_{X} & =\left(\psi_{x}+f_{x} \psi_{u}\right) \Upsilon_{X} \\
& =\mathrm{D}_{x}(\psi) \Upsilon_{X} \\
& =\frac{\mathrm{D}_{x}(\psi)}{\mathrm{D}_{x}(\varphi)}
\end{aligned}
$$

Forgetting the functions $f(x)$ and $F(X)$ which represent graphs, and replacing their firstorder derivatives $f_{x}$ and $F_{X}$ by our independent jet variables $u_{x}$ and $U_{X}$, we have finished to present the basic

Theorem 3.3. [10, 22, 4, 29, 30, 24] When $p=1=q$, every diffeomorphism from $\mathbb{R}_{x}^{1} \times \mathbb{R}_{u}^{1}$ to $\mathbb{R}_{X}^{1} \times \mathbb{R}_{U}^{1}$ :

$$
\phi: \quad(x, u) \longmapsto(X(x, u), U(x, u))
$$

possesses a lift as a diffeomorphism

$$
\phi^{(1)}: \quad\left(x, u, u_{x}\right) \longmapsto\left(X(x, u), U(x, u), U_{X}\left(x, u, u_{x}\right)\right)
$$

from the open subset $G J_{x, u}^{1}$ (graphed jets) of $J_{x, u}^{1}$ defined by

$$
0 \neq \mathrm{D}_{x}(X)=X_{x}+u_{x} X_{u}
$$

(1) (automatic) making commutative the diagram:

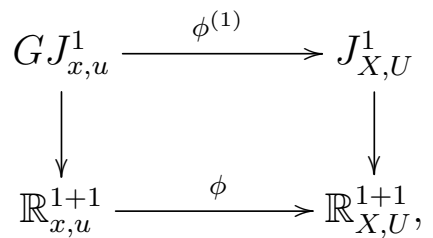

(2) preserving the contact ideal, i.e. $d U-U_{X} d X=(*)\left(d u-u_{x} d x\right)$, where (*) is a nowhere zero function, 
uniquely determined by the formula:

$$
U_{X}=\frac{\mathrm{D}_{x}(U)}{\mathrm{D}_{x}(X)}=\frac{U_{x}+u_{x} U_{u}}{X_{x}+u_{x} X_{u}}
$$

For higher jet orders:

$$
U_{X X}=\frac{1}{\mathrm{D}_{x}(X)} \mathrm{D}_{x}\left(U_{X}\right), \quad U_{X X X}=\frac{1}{\mathrm{D}_{x}(X)} \mathrm{D}_{x}\left(U_{X X}\right),
$$

We do not explain how iterations of the first-order jet formula $U_{X}=\frac{\mathrm{D}_{x}(U)}{\mathrm{D}_{x}(X)}$ provide higher order jets formulas. Once the case $p=q=1$ has been understood, the technicalities concerning higher dimensional cases (involving many indices) become more accessible.

Indeed, coming back to the compact linear system for the partial derivatives of the $\Upsilon^{i}$ left above, using the notation $X^{j}(x, u)$ instead of $\varphi^{j}(x, u)$, we see that this system can be rewritten in matrix form as:

$$
\left(\begin{array}{cccc}
1 & 0 & \cdots & 0 \\
0 & 1 & \cdots & 0 \\
\vdots & \vdots & \ddots & \vdots \\
0 & 0 & \cdots & 1
\end{array}\right)=\left(\begin{array}{cccc}
\Upsilon_{X^{1}}^{1} & \Upsilon_{X^{1}}^{2} & \cdots & \Upsilon_{X^{1}}^{p} \\
\Upsilon_{X^{2}}^{1} & \Upsilon_{X^{2}}^{2} & \cdots & \Upsilon_{X^{2}}^{p} \\
\vdots & \vdots & \ddots & \vdots \\
\Upsilon_{X^{p}}^{1} & \Upsilon_{X^{p}}^{2} & \cdots & \Upsilon_{X^{p}}^{p}
\end{array}\right) \cdot\left(\begin{array}{cccc}
\mathrm{D}_{x^{1}}\left(X^{1}\right) & \mathrm{D}_{x^{1}}\left(X^{2}\right) & \cdots & \mathrm{D}_{x^{1}}\left(X^{p}\right) \\
\mathrm{D}_{x^{2}}\left(X^{1}\right) & \mathrm{D}_{x^{2}}\left(X^{2}\right) & \cdots & \mathrm{D}_{x^{2}}\left(X^{p}\right) \\
\vdots & \vdots & \ddots & \vdots \\
\mathrm{D}_{x^{p}}\left(X^{1}\right) & \mathrm{D}_{x^{p}}\left(X^{2}\right) & \cdots & \mathrm{D}_{x^{p}}\left(X^{p}\right)
\end{array}\right)
$$

which means that:

$$
\left(\begin{array}{ccc}
\Upsilon_{X^{1}}^{1} & \cdots & \Upsilon_{X^{1}}^{p} \\
\vdots & \ddots & \vdots \\
\Upsilon_{X^{p}}^{1} & \cdots & \Upsilon_{X^{p}}^{p}
\end{array}\right)=\left(\begin{array}{ccc}
\mathrm{D}_{x^{1}}\left(X^{1}\right) & \cdots & \mathrm{D}_{x^{1}}\left(X^{p}\right) \\
\vdots & \ddots & \vdots \\
\mathrm{D}_{x^{p}}\left(X^{1}\right) & \cdots & \mathrm{D}_{x^{p}}\left(X^{p}\right)
\end{array}\right)^{-1}
$$

We also remind that the nonvanishing of the Jacobian determinant of the map $x \longmapsto$ $X(x, f(x))$ can be re-expressed after a transposition as the invertibility of this matrix:

$$
0 \neq\left.\operatorname{det}\left(\begin{array}{ccc}
\mathrm{D}_{x^{1}}\left(X^{1}\right) & \cdots & \mathrm{D}_{x^{1}}\left(X^{p}\right) \\
\vdots & \ddots & \vdots \\
\mathrm{D}_{x^{p}}\left(X^{1}\right) & \cdots & \mathrm{D}_{x^{p}}\left(X^{p}\right)
\end{array}\right)\right|_{u=f(x), u_{x^{j}}=f_{x^{j}}(x)} .
$$

The geometric meaning of our assumption that graphs are transformed into graphs is that the composition of three maps: lifting to the graph; performing the diffeomorphism; projecting horizontally:

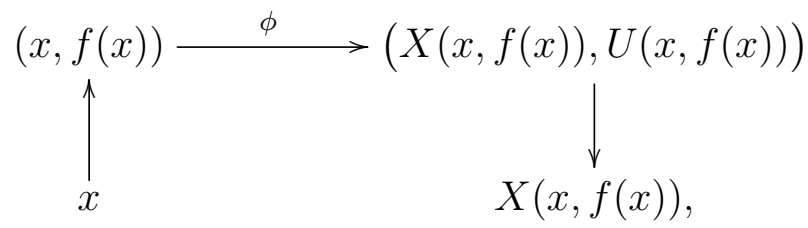

is invertible.

Similarly as we did in the simple case $p=1=q$, we pass the graph $(x, f(x))$ to the target space, and we re-express the result in terms of $X$ :

$$
\begin{aligned}
U^{\alpha} & =\psi^{\alpha}(x, f(x)) \\
& =\psi^{\alpha}(\Upsilon(X), f(\Upsilon(X))) \\
& =: F^{\alpha}(X)
\end{aligned}
$$$$
(1 \leqslant \alpha \leqslant q) .
$$ 
Next, for fixed $\alpha$, we differentiate these equations with respect to $X^{1}, \ldots, X^{p}$ :

$$
\begin{aligned}
& U_{X^{1}}^{\alpha}=\sum_{i=1}^{p} \frac{\partial \psi^{\alpha}}{\partial x^{i}} \frac{\partial \Upsilon^{i}}{\partial X^{1}}+\sum_{i=1}^{p} \sum_{\beta=1}^{q} \frac{\partial \psi^{\alpha}}{\partial u^{\beta}} \frac{\partial f^{\beta}}{\partial x^{i}} \frac{\partial \Upsilon^{i}}{\partial X^{1}}, \\
& U_{X^{p}}^{\alpha}=\sum_{i=1}^{p} \frac{\partial \psi^{\alpha}}{\partial x^{i}} \frac{\partial \Upsilon^{i}}{\partial X^{p}}+\sum_{i=1}^{p} \sum_{\beta=1}^{q} \frac{\partial \psi^{\alpha}}{\partial u^{\beta}} \frac{\partial f^{\beta}}{\partial x^{i}} \frac{\partial \Upsilon^{i}}{\partial X^{p}},
\end{aligned}
$$

that is to say after reorganization:

$$
\begin{gathered}
U_{X^{1}}^{\alpha}=\sum_{i=1}^{p} \frac{\partial \Upsilon^{i}}{\partial X^{1}}\left(\frac{\partial \psi^{\alpha}}{\partial x^{i}}+\sum_{\beta=1}^{q} \frac{\partial f^{\beta}}{\partial x^{i}} \frac{\partial \psi^{\alpha}}{\partial u^{\beta}}\right), \\
\ldots \ldots \ldots \ldots \ldots \ldots \ldots \ldots \ldots \ldots \ldots \ldots \ldots \ldots \ldots \ldots \ldots \ldots \ldots \\
U_{X^{p}}^{\alpha}=\sum_{i=1}^{p} \frac{\partial \Upsilon^{i}}{\partial X^{p}}\left(\frac{\partial \psi^{\alpha}}{\partial x^{i}}+\sum_{\beta=1}^{q} \frac{\partial f^{\beta}}{\partial x^{i}} \frac{\partial \psi^{\alpha}}{\partial u^{\beta}}\right) .
\end{gathered}
$$

Coming back to the notation $U(x, u)$ instead of $\psi(x, u)$, and recognizing the actions of the total differentiation operators, we get, still for any fixed $1 \leqslant \alpha \leqslant q$ :

$$
\left(\begin{array}{c}
U_{X^{1}}^{\alpha} \\
\vdots \\
U_{X^{p}}^{\alpha}
\end{array}\right)=\left(\begin{array}{ccc}
\Upsilon_{X^{1}}^{1} & \cdots & \Upsilon_{X^{1}}^{p} \\
\vdots & \ddots & \vdots \\
\Upsilon_{X^{p}}^{1} & \cdots & \Upsilon_{X^{p}}^{p}
\end{array}\right)\left(\begin{array}{c}
\mathrm{D}_{x^{1}}\left(U^{\alpha}\right) \\
\vdots \\
\mathrm{D}_{x^{p}}\left(U^{\alpha}\right)
\end{array}\right)
$$

We can now state the fundamental general theorem, recalling how we denote coordinates:

$$
\left(x^{i}, u^{\alpha}, u_{x^{j}}^{\beta}\right) \in J_{x, u}^{1} \quad \text { and } \quad\left(X^{i}, U^{\alpha}, U_{X^{j}}^{\beta}\right) \in J_{X, U}^{1},
$$

with trivial projections from the first jet spaces onto the ground manifolds:

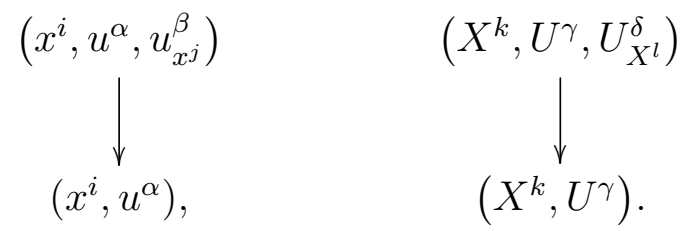

Theorem 3.4. [22, 4, 29, 30, 24] For any $p \geqslant 1$ and any $q \geqslant 1$, every diffeomorphism:

$$
\begin{aligned}
\phi: \mathbb{R}_{x, u}^{p+q} & \longrightarrow \mathbb{R}_{X, U}^{p+q} \\
\left(x^{i}, u^{\alpha}\right) & \longmapsto\left(X^{k}\left(x^{i}, u^{\alpha}\right), U^{\gamma}\left(x^{i}, u^{\alpha}\right)\right),
\end{aligned}
$$

possesses a lift as a diffeomorphism

$$
\phi^{(1)}: \quad\left(x^{i}, u^{\alpha}, u_{x^{j}}^{\beta}\right) \longmapsto\left(X^{k}\left(x^{i}, u^{\alpha}\right), U^{\gamma}\left(x^{i}, u^{\alpha}\right), U_{X^{l}}^{\delta}\left(x^{i}, u^{\alpha}, u_{x^{j}}^{\beta}\right)\right)
$$

from the open subset $G J_{x, u}^{1}$ of $J_{x, u}^{1}$ defined by

$$
0 \neq \operatorname{det}\left(\begin{array}{ccc}
\mathrm{D}_{x^{1}}\left(X^{1}\right) & \cdots & \mathrm{D}_{x^{1}}\left(X^{p}\right) \\
\vdots & \ddots & \vdots \\
\mathrm{D}_{x^{p}}\left(X^{1}\right) & \cdots & \mathrm{D}_{x^{p}}\left(X^{p}\right)
\end{array}\right)
$$


(1) (automatic) making commutative the diagram:

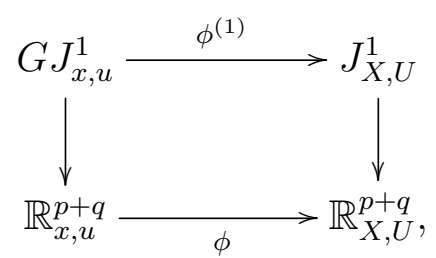

(2) preserving the contact ideal, i.e.

$$
\left(\begin{array}{c}
d U^{1}-U_{X^{1}}^{1} d X^{1} \\
\vdots \\
d U^{q}-U_{X^{p}}^{q} d X^{p}
\end{array}\right)=\left(\begin{array}{ccc}
* & \cdots & * \\
\vdots & \ddots & \vdots \\
* & \cdots & *
\end{array}\right) \cdot\left(\begin{array}{c}
d u^{1}-u_{x^{1}}^{1} d x^{1} \\
\vdots \\
d u^{q}-u_{x^{p}}^{q} d x^{p}
\end{array}\right)
$$

where the transition matrix is everywhere invertible,

uniquely determined by the formula:

$$
\left(\begin{array}{c}
U_{X^{1}}^{\alpha} \\
\vdots \\
U_{X^{p}}^{\alpha}
\end{array}\right)=\left(\begin{array}{ccc}
\mathrm{D}_{x^{1}}\left(X^{1}\right) & \cdots & \mathrm{D}_{x^{1}}\left(X^{p}\right) \\
\vdots & \ddots & \vdots \\
\mathrm{D}_{x^{p}}\left(X^{1}\right) & \cdots & \mathrm{D}_{x^{p}}\left(X^{p}\right)
\end{array}\right)^{-1} \quad\left(\begin{array}{c}
\mathrm{D}_{x^{1}}\left(U^{\alpha}\right) \\
\vdots \\
\mathrm{D}_{x^{p}}\left(U^{\alpha}\right)
\end{array}\right)
$$

For higher jet orders, with $J=j_{1}, \ldots, j_{\lambda}$, with $1 \leqslant j_{1}, \ldots, j_{\lambda} \leqslant p$ arbitrary, and with $1 \leqslant \alpha \leqslant q$ :

$$
\left(\begin{array}{c}
U_{X^{J} X^{1}}^{\alpha} \\
\vdots \\
U_{X^{J} X^{p}}^{\alpha}
\end{array}\right)=\left(\begin{array}{ccc}
\mathrm{D}_{x^{1}}\left(X^{1}\right) & \cdots & \mathrm{D}_{x^{1}}\left(X^{p}\right) \\
\vdots & \ddots & \vdots \\
\mathrm{D}_{x^{p}}\left(X^{1}\right) & \cdots & \mathrm{D}_{x^{p}}\left(X^{p}\right)
\end{array}\right)^{-1} \quad\left(\begin{array}{c}
\mathrm{D}_{x^{1}}\left(U_{X^{J}}^{\alpha}\right) \\
\vdots \\
\mathrm{D}_{x^{p}}\left(U_{X^{J}}^{\alpha}\right)
\end{array}\right) .
$$

Again, we do not provide explanations on how iterations of the first-order jet formula written above conduct to the higher order jet formulas, leaving the details - which can be found in [4] — as an exercise to the interested reader.

Introducing the modified total differentiation operators:

$$
\left(\begin{array}{c}
\mathrm{E}_{x^{1}} \\
\vdots \\
\mathrm{E}_{x^{p}}
\end{array}\right):=\left(\begin{array}{ccc}
\mathrm{D}_{x^{1}}\left(X^{1}\right) & \cdots & \mathrm{D}_{x^{1}}\left(X^{p}\right) \\
\vdots & \ddots & \vdots \\
\mathrm{D}_{x^{p}}\left(X^{1}\right) & \cdots & \mathrm{D}_{x^{p}}\left(X^{p}\right)
\end{array}\right)^{-1}\left(\begin{array}{c}
\mathrm{D}_{x^{1}} \\
\vdots \\
\mathrm{D}_{x^{p}}
\end{array}\right),
$$

the first prolongation of the diffeomorphism $\phi$ rewrites as:

$$
U_{X^{j}}^{\alpha}=\mathrm{E}_{x^{j}}\left(U^{\alpha}\right) \text {. }
$$

Theorem 3.5. For all $1 \leqslant \alpha \leqslant q$ and $1 \leqslant j_{1}, \ldots, j_{\lambda} \leqslant p$ :

$$
U_{X^{j_{1} \ldots X^{j_{\lambda}}}}^{\alpha}=\mathrm{E}_{x^{j_{1}}}\left(\cdots\left(\mathrm{E}_{x^{j_{\lambda}}}\left(U^{\alpha}\right)\right) \cdots\right) .
$$

3.6. Prolongations of vector fields to jet spaces. So we agree that every (local) diffeomorphism $\phi:(x, u) \longmapsto(X(x, u), U(x, u))$ has uniquely determined lifts $\phi^{(n)}$ to the jet spaces of any order $n \geqslant 1$, even if the explicit formulas for the components of $\phi^{(n)}$ are very unwieldy, due to the inversion of the matrix $\mathrm{D}_{x^{i}}\left(X^{j}\right)$, and due to the well known exponential symbolic swelling of formulas through iterated differentiations. Fortunately, as discovered by Lie, linearization of $\phi^{(n)}$ sheds new light, and simplifies the formulas. 
Indeed, every vector field on the base:

$$
\mathrm{v}=\sum_{i=1}^{p} \xi^{i}(x, u) \frac{\partial}{\partial x^{i}}+\sum_{\alpha=1}^{q} \varphi^{\alpha}(x, u) \frac{\partial}{\partial u^{\alpha}},
$$

produces by integration a 1-parameter group of diffeomorphisms:

$$
\phi_{t}(x, u):=\exp (t \mathbf{v})(x, u)
$$

$(t \in \mathbb{R}$ small $)$,

satisfying by definition:

$$
\left.\frac{d}{d t}\right|_{t=0} \phi_{t}(x, u)=\left.\mathbf{v}\right|_{(x, u)} .
$$

Of course, each $\phi_{t}$ possesses lifts to domains of graphed jets of any order $n \geqslant 1$ :

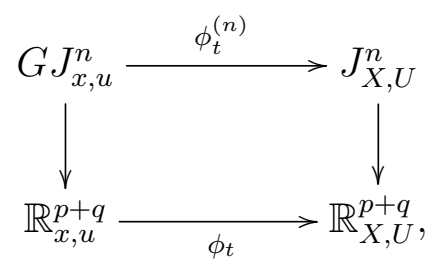

and it comes naturally to mind to differentiate such a lifted 1-parameter family $\phi_{t}^{(n)}$ of diffeomorphisms, obtaining a uniquely determined vector field upstairs:

$$
\mathrm{v}^{(n)}:=\left.\frac{d}{d t}\right|_{t=0} \phi_{t}^{(n)}\left(x^{i}, u^{\alpha}, u_{x^{J}}^{\beta}\right) .
$$

Without recalling the explanations which can be found in the literature, employing again the $p$ total differentiation operators:

$$
\mathrm{D}_{x^{i}}=\frac{\partial}{\partial x^{i}}+\sum_{\alpha=1}^{q} u_{i}^{\alpha} \frac{\partial}{\partial u^{\alpha}}+\sum_{\# J \geqslant 1} \sum_{\alpha=1}^{q} u_{J, i}^{\alpha} \frac{\partial}{\partial u_{J}^{\alpha}}
$$

we shall admit the following very useful theorem which enables to compute the coefficients $\varphi_{J}^{\alpha}$ of these (infinitely) prolonged vector field:

$$
\mathrm{v}^{(\infty)}=\mathrm{v}+\sum_{\# J \geqslant 1} \sum_{\alpha=1}^{q} \varphi_{J}^{\alpha}\left(x^{i}, u^{\beta}, u_{x^{K}}^{\gamma}\right) \frac{\partial}{\partial u_{J}^{\alpha}} .
$$

Theorem 3.7. [22, 29, 30, 24] For any $1 \leqslant \alpha \leqslant q$ and every $J=j_{1}, \ldots, j_{\lambda}$ with $1 \leqslant j_{\nu} \leqslant$ p, one has:

$$
\varphi_{J}^{\alpha}=\mathrm{D}_{x^{J}}\left(\varphi^{\alpha}-\sum_{1 \leqslant i \leqslant p} \xi^{i} u_{i}^{\alpha}\right)+\sum_{1 \leqslant i \leqslant p} \xi^{i} u_{J, i}^{\alpha} .
$$

Here of course, we define:

$$
\mathrm{D}_{x^{j}}:=\mathrm{D}_{x^{j_{1}}} \cdots \mathrm{D}_{x^{j_{\lambda}}}
$$

where the order does not matter, since these total differentiation operators commute. It is important to point out that, due to the obvious relation:

$$
\mathrm{D}_{x^{J}}\left(u_{i}^{\alpha}\right)=u_{J, i}^{\alpha},
$$

the last $p$ terms $\xi_{i} u_{J, i}^{\alpha}$ in the formula for $\varphi_{J}^{\alpha}$ which incorporate jets of order $\# J+1$ do in fact disappear, hence all $\varphi_{J}^{\alpha}$ are functions of jet variables of order $\leqslant \# J$, as they must be. 
The above "direct formulas" for the coefficients $\varphi_{J}^{\alpha}$ are quite convenient when working on a computer. Sometimes, alternative formulas proceeding by recurrence happen to be also useful:

$$
\varphi_{J, j}^{\alpha}=\mathrm{D}_{x^{j}}\left(\varphi_{J}^{\alpha}\right)-\sum_{1 \leqslant i \leqslant p} \mathrm{D}_{x^{j}}\left(\xi^{i}\right) u_{J, i}^{\alpha}
$$

These recurrence formulas were closed and synthetized in [24, Chap. II] to produce explicit formulas for all the $\varphi_{J}^{\alpha}$ by means of multiple Kronecker symbols.

\section{The Moving Frame Method}

We now present the moving frame method, developped by Olver and his collaborators ([13, 31, 32, 33, 35, 36]).

4.1. Source and target jet coordinates. We keep the previous notation for the coordinates in the source space:

$$
z=(x, u)=\left(x^{1}, \ldots, x^{p}, u^{1}, \ldots, u^{q}\right),
$$

but instead of $(X, U)$, we will denote the coordinates in the target space by:

$$
w=(y, v)=\left(y^{1}, \ldots, y^{p}, v^{1}, \ldots, v^{q}\right) .
$$

For any jet order $n \geqslant 0$, jet coordinates will accordingly be denoted by:

$$
\begin{aligned}
z^{(n)} & =\left(x^{i}, u^{\alpha}, u_{x^{J}}^{\beta}\right), \\
w^{(n)} & =\left(y^{j}, v^{\gamma}, v_{y^{K}}^{\delta}\right),
\end{aligned}
$$

with $J=j_{1}, \ldots, j_{\lambda}$, with $1 \leqslant \lambda \leqslant n$, with $1 \leqslant j_{1}, \ldots, j_{\lambda} \leqslant p$, and similarly, with $K=k_{1}, \ldots, k_{\mu}$, with $1 \leqslant \mu \leqslant n$, with $1 \leqslant k_{1}, \ldots, k_{\mu} \leqslant p$. Sometimes, we will abbreviate:

$$
m:=p+q .
$$

Later, we will need to renumber the coordinates by increasing length of derivatives:

$$
\left(w_{1}^{(n)}, \ldots, w_{\mathrm{N}}^{(n)}\right)=\left(y^{j}, v^{\gamma}, v_{y^{K_{1}}}^{\delta_{1}}, \ldots, v_{y^{K_{n}}}^{\delta_{n}}\right),
$$

where the total number $\mathrm{N}$ is the dimension of the jet space $J_{p, q}^{n}$ :

$$
\mathrm{N}=\mathrm{N}_{p, q}(n):=p+q\left(\begin{array}{c}
p+n \\
n
\end{array}\right),
$$

where $\# K_{1}=1, \ldots, \# K_{n}=n$, and where we choose any ordering for the subcollection of jet coordinates $v_{y^{K_{h}}}^{\delta_{h}}$ with $1 \leqslant \delta_{h} \leqslant q$ and $\# K_{h}=h$.

The first $p+q$ coordinates $w_{1}^{(n)}, \ldots, w_{p+q}^{(n)}$ belong in fact to the zero ${ }^{\text {th }}$ jet order space, hence should be written $w_{1}^{(0)}, \ldots, w_{p+q}^{(0)}$. By convention, for any jet order $n^{\prime} \geqslant n$, the trivial projection $J_{p, q}^{n^{\prime}} \longrightarrow J_{p, q}^{n}$ allows to identify $w_{k}^{\left(n^{\prime}\right)} \equiv w_{k}^{(n)}$ as soon as $w_{k}^{(n)}$ is a coordinate of $J_{p, q}^{n} \cdot$

4.2. Prolongations of groups actions. Next, assume that a finite-dimensional local Lie group $G$ acts on $\mathbb{R}_{x, u}^{p+q}$, and denote its dimension by:

$$
r:=\operatorname{dim} G
$$

$(1 \leqslant r<\infty)$. 
Indeed, it is convenient (and theoretically wider) to assume that $G$ is a local Lie group, as did Lie in [10, 22]. Therefore, the diffeomorphisms $\phi: \mathbb{R}_{x, u}^{p+q} \longrightarrow \mathbb{R}_{y, v}^{p+q}$ we are considering are 'parametrized' by group elements $g \in G$ in some neighborhood of the identity element:

$$
e \in G,
$$

and local Lie group axioms about composition and inversion hold. As we will not require background reminder about foundational concepts of Lie theory, the interested reader will be referred to [22, pp. 29-32].

We will not employ functional symbols $\phi=(\varphi, \psi)$ like in Section 3 , but instead, we will denote diffeomorphisms 'parametrized' by group elements $g \in G$ as Lie did:

$$
y=y(g, x, u), \quad v=v(g, x, u) .
$$

Sometimes, we will also use the more compact notation $w=g \cdot z$ instead of $w=w(g, x, u)$.

Definition 4.3. The action of $G$ on $\mathbb{R}_{x, u}^{p+q}$ is said to be free if the isotropy subgroup of every $z=(x, u)$ reduces to the identity:

$$
\{e\}=\{g \in G: g \cdot z=z\}=: G_{z} \quad\left(\forall z \in \mathbb{R}^{m}\right) .
$$

In general, the action of $G$ on $\mathbb{R}_{x, u}^{p+q}$ is not free, just because as soon as $r \geqslant m+1$, isotropy subgroups $G_{z}$ are of dimension $\geqslant r-m \geqslant 1$. As soon as the maps $g \longmapsto$ $w(g, x, u)$ are not of full rank $r$, freeness is lost.

A good substitute is to assume that the ranks of the maps $g \longmapsto w(g, x, u)$ are constant. In this case, after perhaps passing to smaller open subsets, the $G$-orbits of the local Lie group $G$ are of constant dimension, and their union foliates some open set in $\mathbb{R}_{x, u}^{p+q}$. In the analytic category, group actions are generically of local constant rank, in appropriate subdomains which have an invariant meaning, hence rank constancy is essentially no assumption in Lie theory. This principle of thought is explained in Chapter 1 of [22].

All these observations remain valid in jet spaces of any order $n \geqslant 0$, because thanks to Theorem 3.4, every group-diffeomorphism $z \longmapsto g \cdot z$ (close the identity mapping) lifts upstairs as a jet diffeomorphism:
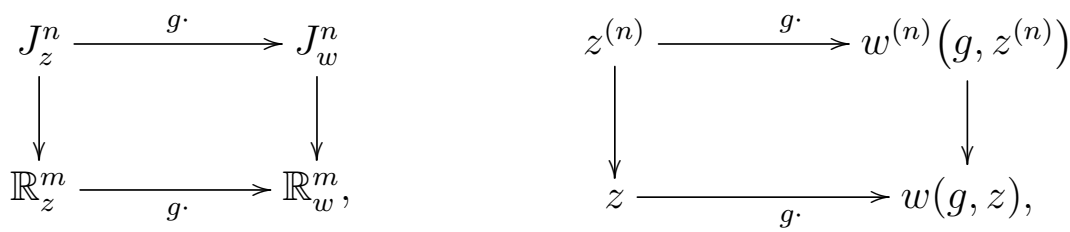

vertical arrows being (trivial) projections, as before. The advantage of prolongating is that the ambient dimension increases polynomially with $n$ :

$$
\operatorname{dim} J_{p, q}^{n}=p+q\left(\begin{array}{c}
p+n \\
n
\end{array}\right)
$$


and hence, granted that prolongation commutes with projections from higher order $n^{\prime} \geqslant$ $n \geqslant 0$ jet spaces:

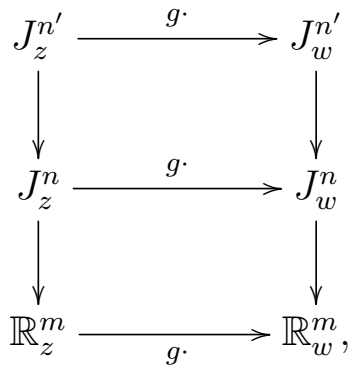

it is clear that the (generic) ranks of the maps $g \longmapsto w^{(n)}\left(g, z^{(n)}\right)$ are increasing with $n$. Of course, the ranks of these maps $g \longmapsto w^{(n)}\left(g, z^{(n)}\right)$ are considered for $g \sim e$ close to the identity element, and for $z^{(n)}$ in $J_{p, q}^{(n)}$, or in some appropriate open subsets of $J_{p, q}^{(n)}-$ more will be said soon about that.

We shall use the abbreviation:

$$
\mathrm{N}:=\mathrm{N}_{p, q}(n):=p+q\left(\begin{array}{c}
p+n \\
n
\end{array}\right) .
$$

For most of the existing local Lie group actions (and for all the ones studied in the present article), there always exists a minimal finite jet order:

$$
0 \leqslant n_{\mathrm{G}}<\infty
$$

such that:

$$
\operatorname{rank}\left(g \longmapsto w^{\left(n_{\mathrm{G}}\right)}\left(g, z^{\left(n_{\mathrm{G}}\right)}\right)\right)=r=\operatorname{dim} G,
$$

and hence the action of the local Lie group $G$ is free. A necessary — and often sufficient condition is that:

$$
p+q\left(\begin{array}{c}
p+n_{\mathrm{G}} \\
n_{\mathrm{G}}
\end{array}\right)=\mathrm{N}_{p, q}\left(n_{\mathrm{G}}\right) \geqslant r .
$$

Example 4.4. Let the affine group $\mathrm{A}_{2}(\mathbb{R})$ on the plane $\mathbb{R}_{x, u}^{2}$ have general transformations $y=\mathrm{a} x+\mathrm{b} u+\mathrm{c}$ and $v=\mathrm{k} x+\mathrm{I} u+\mathrm{m}$ with $0 \neq\left|\begin{array}{l}\mathrm{a} \\ \mathrm{k}\end{array}\right| \mathrm{l} \mid$. With the modified total differentiation operator:

$$
\mathrm{E}_{x}:=\frac{1}{\mathrm{a}+\mathrm{b} u_{x}}\left[\frac{\partial}{\partial x}+u_{x} \frac{\partial}{\partial u}+\sum_{i=1}^{\infty} u_{x^{i+1}} \frac{\partial}{\partial u_{x^{i}}}\right],
$$

an application of Theorem 3.5 yields the second prolongation on graphed curves $\{u=$ $u(x)\}$ of such affine transformations:

$$
v_{y y}=\mathrm{E}_{x}\left(\mathrm{E}_{x}(v)\right)=\frac{\mathrm{al}-\mathrm{bk}}{\left(\mathrm{a}+\mathrm{b} u_{x}\right)^{3}} u_{x x} .
$$

We readily see that the condition $u_{x x} \neq 0$ is affinely invariant, hence the second order jet space stratifies $A_{2}(\mathbb{R})$-invariantly as:

$$
J_{x, u}^{2}=\left\{u_{x x}=0\right\} \cup\left\{u_{x x} \neq 0\right\} .
$$

Of course, the flatness condition $u_{x x}(x) \equiv 0$ which means that the graph $u(x)=\lambda x+\mu$ being a straight line is affinely invariant! 
4.5. Foliated $G$-actions and differential invariants. For general actions of finitedimensional Lie groups on graphs in $\mathbb{R}_{x, u}^{p+q}$, a case-by-case study is required to determine the stratification of jet spaces $J_{x, u}^{n}$ in appropriate invariant pieces wherein the lifted action of $G$ has constant rank properties.

In later sections, we will conduct a precise stratification study for parabolic surfaces $S^{2} \subset \mathbb{R}^{3}$.

Definition 4.6. A lifted action of a local Lie group $G$ to a jet space $J_{p, q}^{n}$ is called foliated at a point $z_{0}^{(n)} \in J_{p, q}^{n}$ when all $G$-orbits have constant dimension equal to a certain integer $s$ with $0 \leqslant s \leqslant r=\operatorname{dim} G$ in a neighborhood of $z_{0}^{(n)}$, with maps $g \longmapsto w^{(n)}\left(g, z^{(n)}\right)$ having constant rank $s$ for all $z^{(n)}$ near $z_{0}^{(n)}$ and all $g$ near $e \in G$.

Under this assumption, the rank theorem guarantees that the collection of $G$-orbits indeed constitutes a local foliation by $s$-dimensional manifolds in a neighborhood of $z_{0}^{(n)}$.

Most of the time, for any action of a local Lie group $G$ on graphs $\{u=u(x)\}$ in $\mathbb{R}_{x, u}^{p+q}$, the induced action of $G$ on some jet space $J_{x, u}^{n}$ (even of low order) becomes almost everywhere foliated, although not necessarily free.
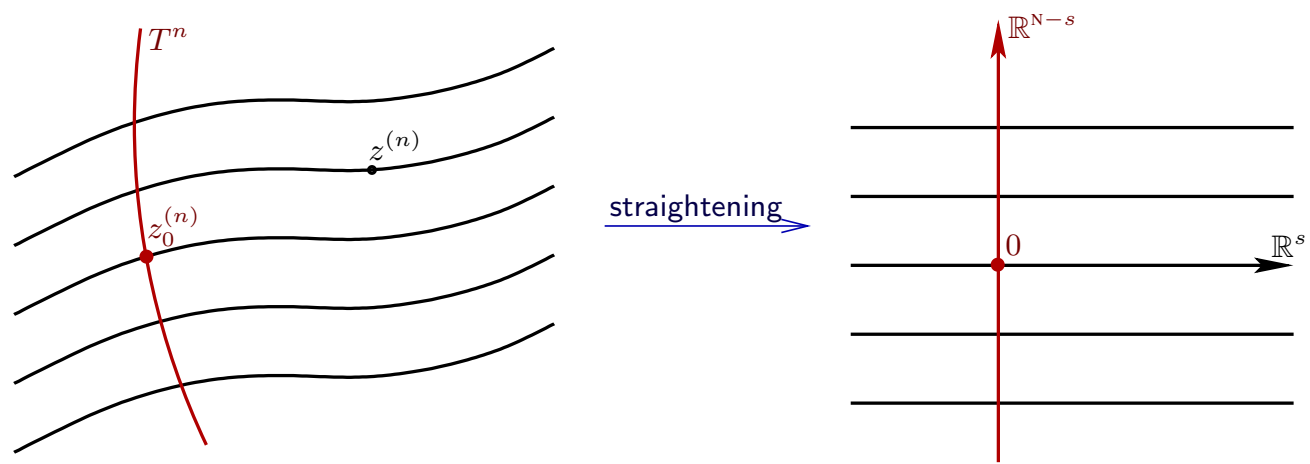

Assuming the action of $G$ is foliated at some $z_{0}^{(n)} \in J_{p, q}^{n}$, let us choose a transversal $T^{n}$ to the $G$-orbits at $z_{0}^{(n)}$, namely a local submanifold:

$$
z_{0}^{(n)} \in T^{n} \subset J_{p, q}^{n}
$$

of dimension complementary to the dimension of $G$-orbits:

$$
\operatorname{dim} T^{n}=\mathrm{N}_{p, q}(n)-s,
$$

which is also transversal to the $G$-orbit $G \cdot z_{0}^{(n)}$ in the sense of transversality theory:

$$
T_{z_{0}^{(n)}} T^{n} \oplus T_{z_{0}^{(n)}} G \cdot z_{0}^{(n)}=T_{z_{0}^{(n)}} J_{p, q}^{n} .
$$

After a local straightening diffeomorphism, we can make $G$-orbits horizontal, directed by $\mathbb{R}^{s} \times\{0\}$, and the transversal $T^{n}$ vertical as well, directed by $\{0\} \times \mathbb{R}^{\mathrm{N}-s}$. This means that we can get $\mathrm{N}-s$ functions:

$$
I_{1}\left(z^{(n)}\right), \ldots \ldots, I_{\mathrm{N}-s}\left(z^{(n)}\right),
$$

vanishing at $z_{0}^{(n)}$, such that the union of $G$-orbits near $z_{0}^{(n)}$ is represented as:

$$
\bigcup_{c_{1}, \ldots, c_{\mathrm{N}-s}}\left\{I_{1}=c_{1}, \ldots, I_{\mathrm{N}-s}=c_{\mathrm{N}-s}\right\},
$$


with arbitrary constants $c_{1}, \ldots, c_{\mathrm{N}-s}$ all close to 0 . In general, producing such functions $I_{1}, \ldots, I_{\mathrm{N}-s}$ requires an application of the implicit function theorem. Hence explicitness can be lost.

Certainly, such $G$-invariants $I_{1}, \ldots, I_{\mathrm{N}-s}$ are functionally independent in the sense that:

$$
\mathscr{R}\left(I_{1}\left(z^{(n)}\right), \ldots, I_{\mathrm{N}-s}\left(z^{(n)}\right)\right) \equiv 0 \quad \Longrightarrow \quad \mathscr{R}=0
$$

because this property is trivially true after straightening, and is invariant under diffeomorphisms. Equivalently, the differentials of these invariants are linearly independent:

$$
0 \neq d \boldsymbol{I}_{1}\left(z^{(n)}\right) \wedge \cdots \wedge d \boldsymbol{I}_{\mathrm{N}-s}\left(z^{(n)}\right) \quad\left(\forall z^{(n)} \text { near } z_{0}^{(n)}\right) .
$$

Definition 4.7. A (local) differential invariant $I: J_{p, q}^{n} \longrightarrow \mathbb{R}$ is a function defined in a neighborhood of some $z_{0}^{(n)} \in J_{p, q}^{n}$ which is constant on (local) $G$-orbits:

$$
I\left(g \cdot z^{(n)}\right)=I\left(z^{(n)}\right) \quad(\forall g \in G)
$$

Of course, $G$ is a local Lie group as before. In true applications, as we will soon e.g. in the context of parabolic surfaces $S^{2} \subset \mathbb{R}^{3}$, the behavior of differential invariants, their number, their syzygies, will be constant in certain Zariski open subsets of the jet spaces $J_{p, q}^{n}$, for every $n \geqslant 0$, and it is a part of an adequate mathematical work to determine appropriate invariant stratifications of these $J_{p, q}^{n}$.

Theorem 4.8. [10, 29, 30, 22] Given an action on $\mathbb{R}_{x}^{p} \times \mathbb{R}_{u}^{q}$ of a local Lie group $G$, for any jet order $n \geqslant 0$, given the induced action of $G$ on the space $J_{p, q}^{n}$ of jets of maps $x \longmapsto u(x)$, with $\mathrm{N}=\mathrm{N}_{p, q}(n)=\operatorname{dim} J_{p, q}^{n}$, at any point $z_{0}^{(n)} \in J_{p, q}^{n}$ at which the induced G-action is foliated of rank $s$, with $0 \leqslant s \leqslant r$ being the dimension of leaves, there are exactly $\mathrm{N}_{p, q}(n)-s$ functionally independent differential invariants $I_{1}, \ldots, I_{\mathrm{N}-s}$ defined near $z_{0}^{(n)}$ so that any other differential invariant I is a certain, uniquely defined, function of these:

$$
I=\mathscr{F}\left(I_{1}, \ldots, I_{\mathrm{N}-s}\right)
$$

4.9. Free actions and moving frames. Assume now that $n \geqslant 0$ is large enough so that the induced action of $G$ on $J_{p, q}^{n}$ is locally free near some $z_{0}^{(n)} \in J_{p, q}^{(n)}$, and also foliated. As before, denote by $n_{\mathrm{G}}$ the minimal such $n$. Presently, we will work only with jet orders $n \geqslant n_{\mathrm{G}}$.

Thus, all $G$-orbits on $J_{p, q}^{n}$ have maximal possible dimension $r=\operatorname{dim} G$. Thanks to the preceding Theorem 4.8 , we know that there are exactly $\mathrm{N}_{p, q}(n)-r$ functionally independent differential invariants.

We now take $n:=n_{\mathrm{G}}$ minimal possible, so the number of independent differential invariants is $\mathrm{N}_{p, q}\left(n_{\mathrm{G}}\right)-r$. 


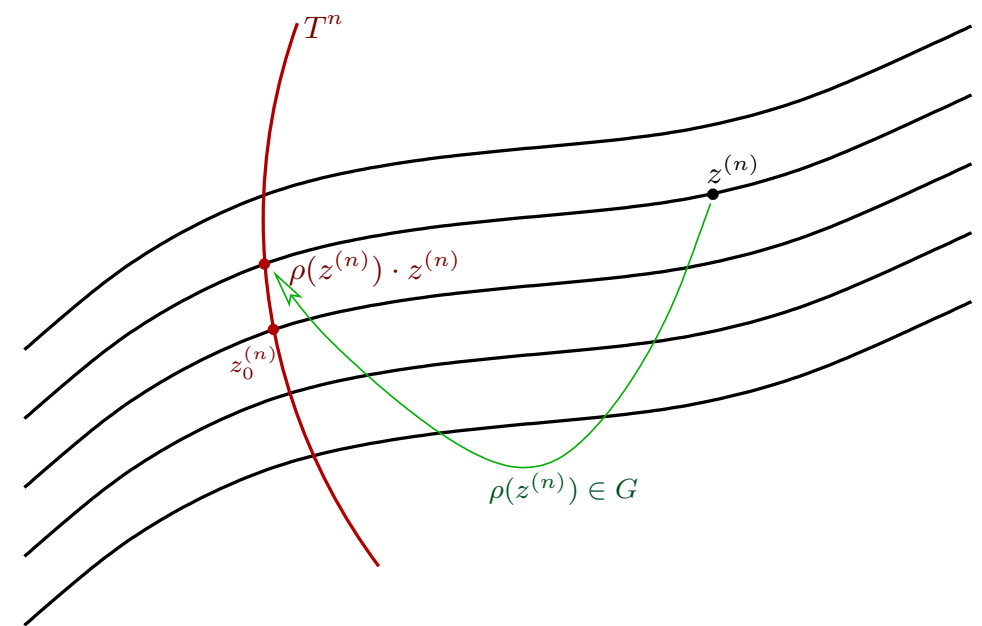

The geometrically evident fact that every local $G$-orbit intersects the transversal $T^{n_{\mathrm{G}}} \subset$ $J_{p, q}^{n_{\mathrm{G}}}$ in a single point explains the:

Lemma 4.10. For every $z^{\left(n_{\mathrm{G}}\right)}$ near $z_{0}^{\left(n_{\mathrm{G}}\right)}$, there exists a unique group element $g \in G$ near $e$ such that:

$$
g \cdot z^{\left(n_{\mathrm{G}}\right)} \in T^{n_{\mathrm{G}}}
$$

As in [13], we will denote this unique group element by $\rho\left(z^{\left(n_{\mathrm{G}}\right)}\right)$, so that:

$$
\rho\left(z^{\left(n_{\mathrm{G}}\right)}\right) \cdot z^{\left(n_{\mathrm{G}}\right)} \in T^{n_{\mathrm{G}}} .
$$

We can be more specific about how the map $\rho: J_{p, q}^{n_{\mathrm{G}}} \longmapsto G$ can be handled in concrete situations. Remind from Subsection 4.1 our notation for coordinates on $J_{p, q}^{n_{\mathrm{G}}}$ :

$$
\left(w_{1}^{\left(n_{\mathrm{G}}\right)}, \ldots, w_{\mathrm{N}_{p, q}\left(n_{\mathrm{G}}\right)}^{\left(n_{\mathrm{G}}\right)}\right)=\left(y^{j}, v^{\gamma}, v_{y^{K_{1}}}^{\delta_{1}}, \ldots, v_{y^{K_{n_{\mathrm{G}}}}}^{\delta_{n_{\mathrm{G}}}}\right) .
$$

A transversal to the $G$-orbits is usually constructed after a detailed study of specific features of the group action.

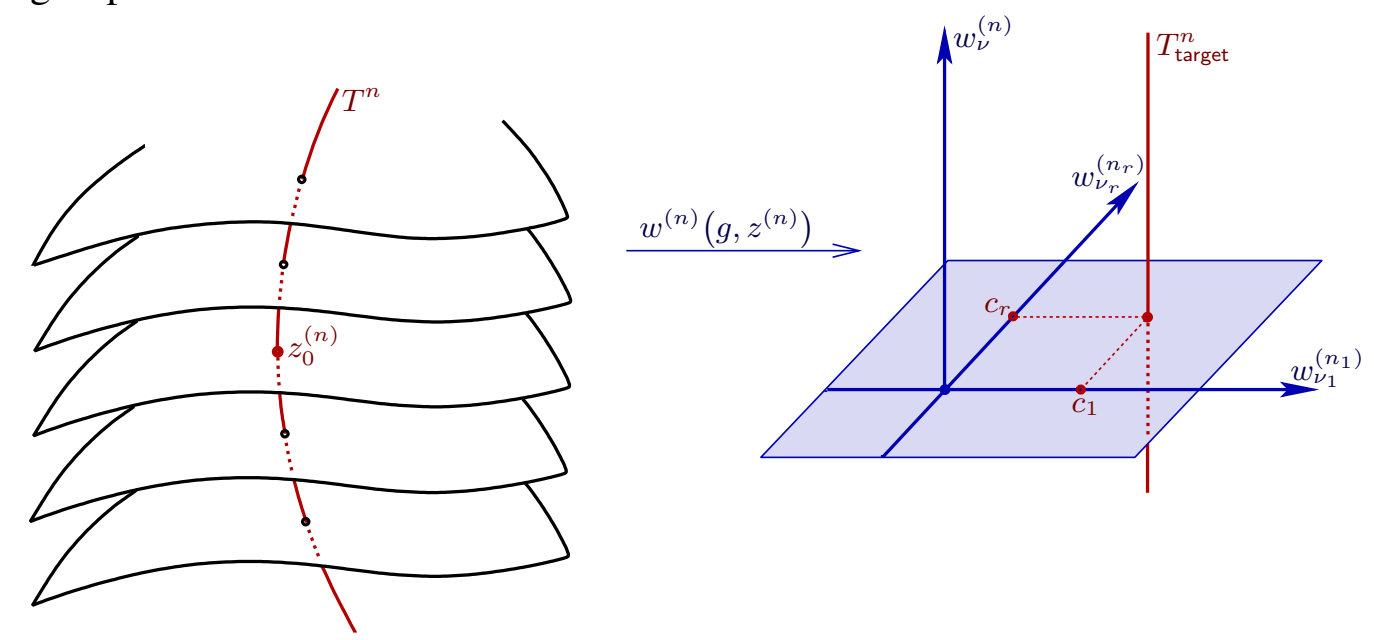

Generally, a number $r=\operatorname{dim} G$ of integers:

$$
1 \leqslant \nu_{1}<\cdots<\nu_{h}<\cdots<\nu_{r} \leqslant \mathrm{~N},
$$

are then 'selected' in some 'natural' way, and constants $c_{1}, \ldots, c_{r} \in \mathbb{R}-$ often equal to 0 or 1 - are also 'chosen' so that, viewed in the target jet space as on the right hand side of 
the figure above, the transversal has defining equations of the shape:

$$
w_{\nu_{1}}^{\left(n_{1}\right)}=c_{1}, \ldots \ldots, w_{\nu_{h}}^{\left(n_{h}\right)}=c_{h}, \ldots \ldots, w_{\nu_{r}}^{\left(n_{r}\right)}=c_{r}
$$

where for $1 \leqslant h \leqslant r$, the integer $\left(n_{h}\right)$ is the minimal jet space order to which the jet coordinate $w_{\nu_{h}}^{\left(n_{\mathrm{G}}\right)}=w_{\nu_{h}}^{\left(n_{h}\right)}$ belongs, and where we have $n_{r}=n_{\mathrm{G}}$, by definition of $n_{\mathrm{G}}$.

Viewed in the source space as on the left of the figure above, the equations of the transversal $T^{n_{\mathrm{G}}} \subset J_{p, q}^{n_{\mathrm{G}}}$ then read by understanding that the $w_{h}^{\left(n_{h}\right)}$ are coordinates of the prolongation of the $G$-action:

$$
w_{\nu_{1}}^{\left(n_{1}\right)}\left(g, z^{\left(n_{1}\right)}\right)=c_{1}, \ldots \ldots \ldots, w_{\nu_{r}}^{\left(n_{r}\right)}\left(g, z^{\left(n_{r}\right)}\right)=c_{r},
$$

and the assumption that $T^{n_{\mathrm{G}}}$ is a transversal is equivalent to the fact that all the group parameters $g=\left(g_{1}, \ldots, g_{r}\right)$ can be solved from these $r$ equations by means of the implicit function theorem:

$$
g=\rho\left(z^{\left(n_{1}\right)}, \ldots, z^{\left(n_{\mathrm{G}}\right)}\right) \equiv \rho\left(z^{\left(n_{\mathrm{G}}\right)}\right)
$$

For every $n \geqslant n_{\mathrm{G}}$, a transversal $T^{n} \subset J_{p, q}^{n}$ can be chosen to have exactly the same equations as a transversal $T^{n_{\mathrm{G}}} \subset J_{p, q}^{n_{\mathrm{G}}}$ in the minimal order $n_{\mathrm{G}}$ jet space as written above. For later applications, e.g. to parabolic surfaces $S^{2} \subset \mathbb{R}^{3}$, we want to keep memory that the map:

$$
z^{(n)} \longmapsto \rho\left(z^{(n)}\right)=\rho\left(z^{\left(n_{\mathrm{G}}\right)}\right)
$$

only depends on jets of this minimal possible order $n_{\mathrm{G}}$. We can now formulate a definition valid for any $n \geqslant n_{\mathrm{G}}$.

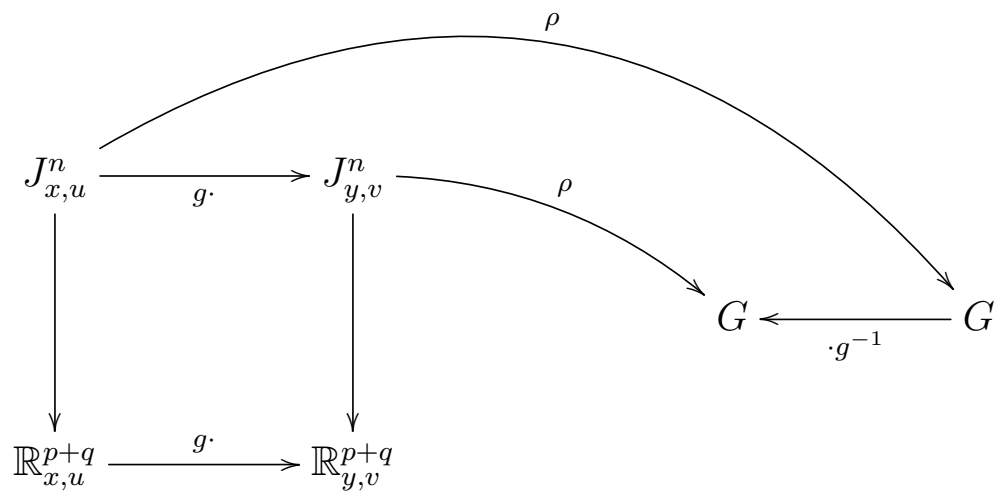

Definition 4.11. [12, 13] A (local) right moving frame for the action on $\mathbb{R}_{x, u}^{p+q}$ of a local Lie group $G$ lifted to an $n^{\text {th }}$ jet space $J_{p, q}^{n}$ is a map:

$$
\rho: \quad J_{p, q}^{n} \longrightarrow G
$$

defined near some jet $z_{0}^{(n)} \in J_{p, q}^{n}$ which satisfies the right equivariancy rule:

$$
\rho\left(g \cdot z^{(n)}\right)=\rho\left(z^{(n)}\right) \cdot g^{-1}
$$

for all $g \in G$ near $e$ and all $z^{(n)}$ near $z_{0}^{(n)}$. 


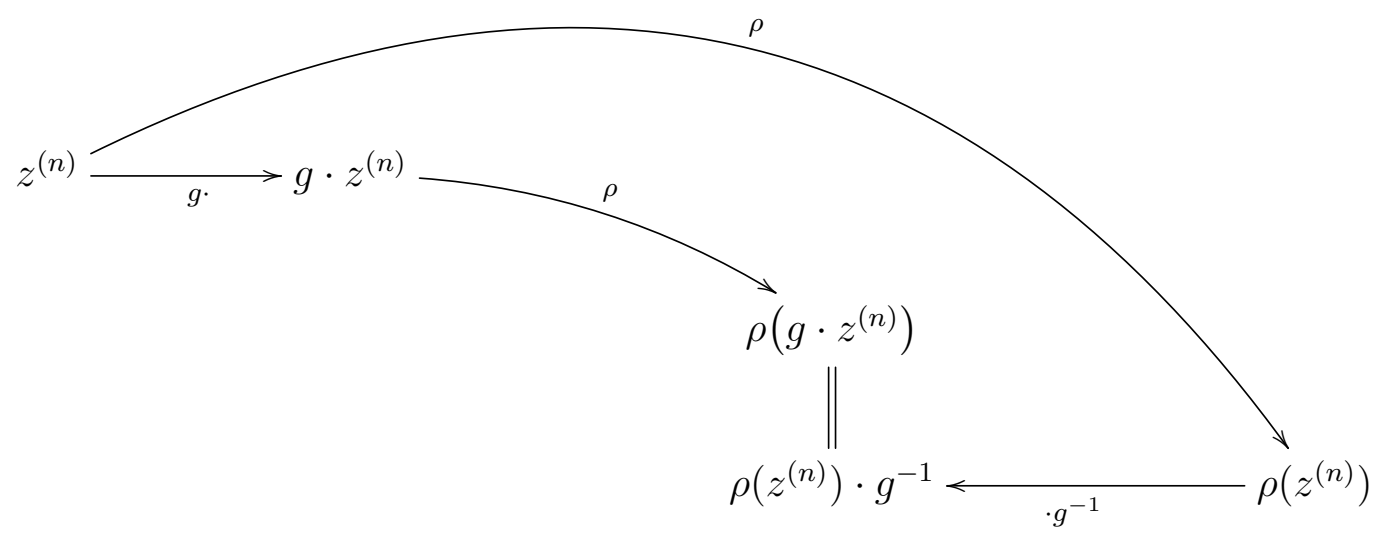

Lemma 4.12. [13, Sec. 4] For any choice of a transveral $T^{n_{\mathrm{G}}} \subset J_{p, q}^{n_{\mathrm{G}}}$ as above, and for any $n \geqslant n_{\mathrm{G}}$, the map constructed above by means of the implicit function theorem:

$$
\rho\left(z^{(n)}\right)=\rho\left(z^{\left(n_{\mathrm{G}}\right)}\right)
$$

automatically satisfies the right equivariancy rule.

The proof, short, will be skipped here, as well as the proof of the next elementary

Theorem 4.13. [13, Sec. 4] If the action of $G$ lifted to $J_{p, q}^{n}$ is foliated at a point $z_{0}^{(n)}$, the following three conditions are equivalent:

(i) a moving frame exists in a neighborhood of $z_{0}^{(n)}$;

(ii) G acts locally freely near $z_{0}^{(n)}$;

(iii) G-orbits have maximal dimension $r=\operatorname{dim} G$ near $z_{0}^{(n)}$.

When studying parabolic surfaces $S^{2} \subset \mathbb{R}^{3}$, with $G=\mathrm{SA}_{3}(\mathbb{R})=\mathrm{SL}_{3}(\mathbb{R}) \ltimes \mathbb{R}^{3}$ having dimension 11, we will see that in some appropriately 'truncated' $4^{\text {th }}$ jet space having also dimension 11, the $G$-orbits have constant dimension $10=11-1$, whence Theorem 4.8 guarantees that there exists one differential invariant of order 4 , which we will call $W$. But then because the rank of the $G$-action degenerates by $1=11-10$ dimension, the above Theorem 4.13 does not apply.

Question 4.14. Is there an appropriate substitute to a moving frame when $G$-orbits have constant dimension $0 \leqslant s \leqslant r-1$ smaller than $\operatorname{dim} G$ ?

One can think of the isotropy subgroup $G_{z_{0}^{(n)}} \subset G$ of a reference point $z_{0}^{(n)}$, but then, in general, this subgroup $H:=G_{z_{0}^{(n)}}$ is not distinguished in $G$, only left cosets $G / H$ or right cosets $H \backslash G$ can be dealt with.

In presence of a moving frame, Theorem 4.8 becomes the

Theorem 4.15. [13, Sec. 4] If $\rho: J_{p, q}^{n} \longrightarrow G$ is a right moving frame, with $n \geqslant n_{\mathrm{G}}$, then in coordinates, all components of the map:

$$
z^{(n)} \longmapsto \rho\left(z^{\left(n_{\mathrm{G}}\right)}\right) \cdot z^{(n)}
$$

constitute a complete generating set of differential invariants of order at most $n$. 
Concretely, after having solved the group parameters $g=\left(g_{1}, \ldots, g_{r}\right)$ from the $r$ equations $w_{\nu_{h}}=c_{h}, 1 \leqslant h \leqslant r$, of some transversal $T^{n_{\mathrm{G}}} \subset J_{p, q}^{n_{\mathrm{G}}}$, one replaces these solutions $g=\rho\left(z^{\left(n_{\mathrm{G}}\right)}\right)$ in all the other coordinate formulas $w_{\nu}\left(g, z^{(n)}\right)$ for the prolonged $G$-action, and one obtains a generating set of $\mathrm{N}_{p, q}(n)-r$ differential invariants of order at most $n$ :

$$
I_{\nu}:=w_{\nu}\left(\rho\left(z^{n_{\mathrm{G}}}\right), z^{(n)}\right)
$$

$\left(1 \leqslant \nu \leqslant \mathrm{N}_{p, q}(n), \nu \neq \nu_{1}, \ldots, \nu_{r}\right)$,

When $n \geqslant 1+n_{\mathrm{G}}$, this means that all indices:

$$
\mathrm{N}_{p, q}\left(n_{\mathrm{G}}\right)+1 \leqslant \nu \leqslant \mathrm{N}_{p, q}(n)
$$

are concerned by such a replacement.

By definition of how the implicit function theorem applies to the equations $w_{\nu_{h}}=c_{h}$, if one replaces $g=\rho\left(z^{\left(n_{\mathrm{G}}\right)}\right)$ in the coordinates $w_{\nu_{1}}, \ldots, w_{\nu_{r}}$ which were used to construct the moving frame, one gets trivial constants:

$$
w_{\nu_{1}}\left(\rho\left(z^{\left(n_{\mathrm{G}}\right)}\right), z^{\left(n_{1}\right)}\right) \equiv c_{1}, \ldots \ldots \ldots, w_{\nu_{r}}\left(\rho\left(z^{\left(n_{\mathrm{G}}\right)}\right), z^{\left(n_{r}\right)}\right) \equiv c_{r}
$$

Terminology 4.16. [13] These $r$ differential quantities $w_{\nu_{h}}\left(\rho\left(z^{\left(n_{\mathrm{G}}\right)}\right), z^{\left(n_{h}\right)}\right) \equiv c_{h}$ are called phantom differential invariants.

These ghost objects will be very useful later.

\section{What it Really Means to Be a Differential Invariant}

As before, let $G$ be a local Lie group acting on graphs $\{u=\mathrm{u}(x)\}$ in $\mathbb{R}_{x}^{p} \times \mathbb{R}_{u}^{q}$, of finite dimension $1 \leqslant r=\operatorname{dim} G<\infty$. The action of an element $g \in G$ lifts to all jet spaces $J_{x, u}^{n}$ of any order $n \geqslant 0$, as in the following diagrams:
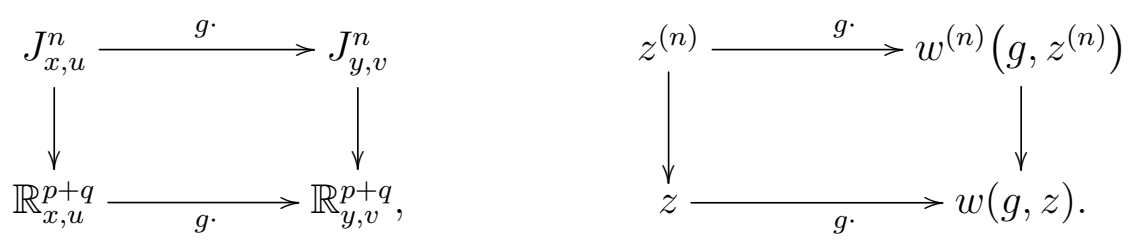

By definition, a differential invariant of order $n$ is a function $I: J_{x, u}^{n} \longrightarrow \mathbb{R}$ satisfying:

$$
I\left(g \cdot z^{(n)}\right)=I\left(z^{(n)}\right)
$$

$(\forall g \in G)$,

a property that can be diagrammatized as follows:
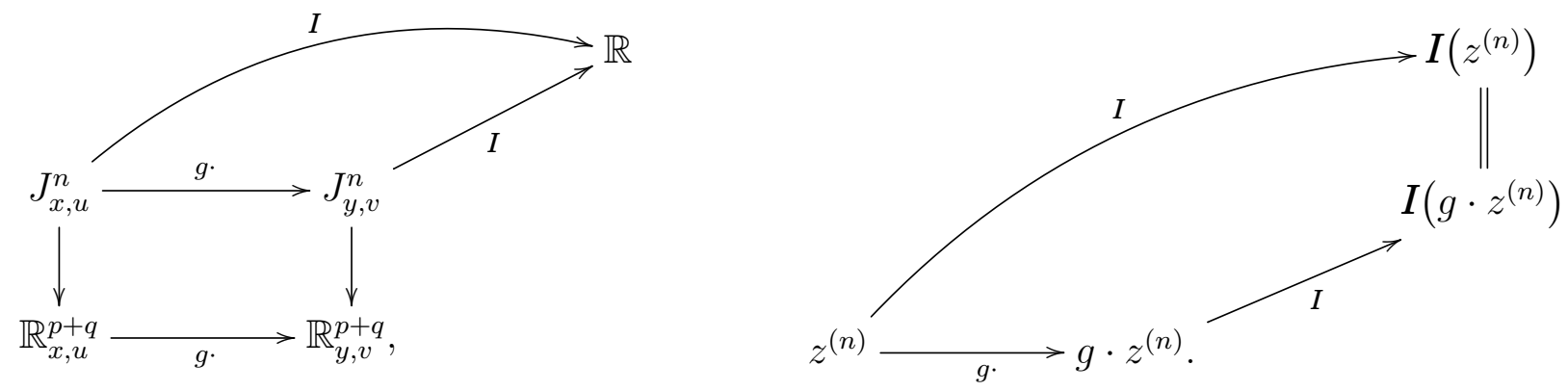

Viewed as such, a differential invariant is a function defined on the jet space $J_{x, u}^{n}$ equipped with independent jet coordinates:

$$
z^{(n)}=\left(x^{j}, u^{\alpha}, u_{x^{j}}^{\beta}\right)
$$

but it has another more interesting meaning. 
Indeed, remember that we are considering graphs $\{u=\mathrm{u}(x)\}$ in the source space and their transforms, which are graphs $\{v=\mathrm{v}(y)\}$ in the target space. So pulled back to any graph $\{u=\mathrm{u}(x)\}$, a differential invariant becomes a function of the coordinates $x=\left(x^{1}, \ldots, x^{p}\right)$ on the graph:

$$
x \longmapsto I\left(x, \mathrm{u}^{\alpha}(x), \mathrm{u}_{x^{J}}^{\beta}(x)\right) .
$$

Hence a differential invariant takes various values at various points of a graphed manifold $\{u=\mathrm{u}(x)\}$.

Question 5.1. Then what does it really mean, for $I\left(x^{j}, u^{\alpha}, u_{x^{J}}^{\beta}\right)$, to be a differential invariant?

Of course, in the target space $\mathbb{R}_{y, v}^{p+q}$, the same function $I$ of the target arguments must be considered:

$$
I\left(y^{k}, v^{\gamma}, v_{y^{K}}^{\delta}\right) .
$$

But what is the relation with $I\left(x^{j}, u^{\alpha}, u_{x^{J}}^{\beta}\right)$ ?

Recall that any diffeomorphism $\phi:(x, u) \longmapsto(\varphi(x, u), \psi(x, u))$ not far from the identity induces a horizontal diffeomorphism between the graphing horizontal spaces $\mathbb{R}_{x}^{p}$ and $\mathbb{R}_{y}^{p}$, simply through three maps: lifting to the graph; performing the diffeomorphism; projecting horizontally:

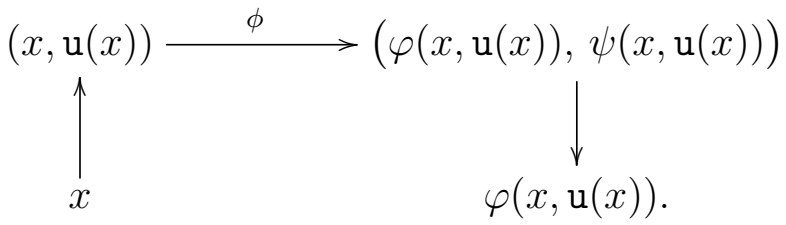

We therefore assume that we have a (local) diffeomorphism $\mathbb{R}_{x}^{p} \longmapsto \mathbb{R}_{y}^{p}$ :

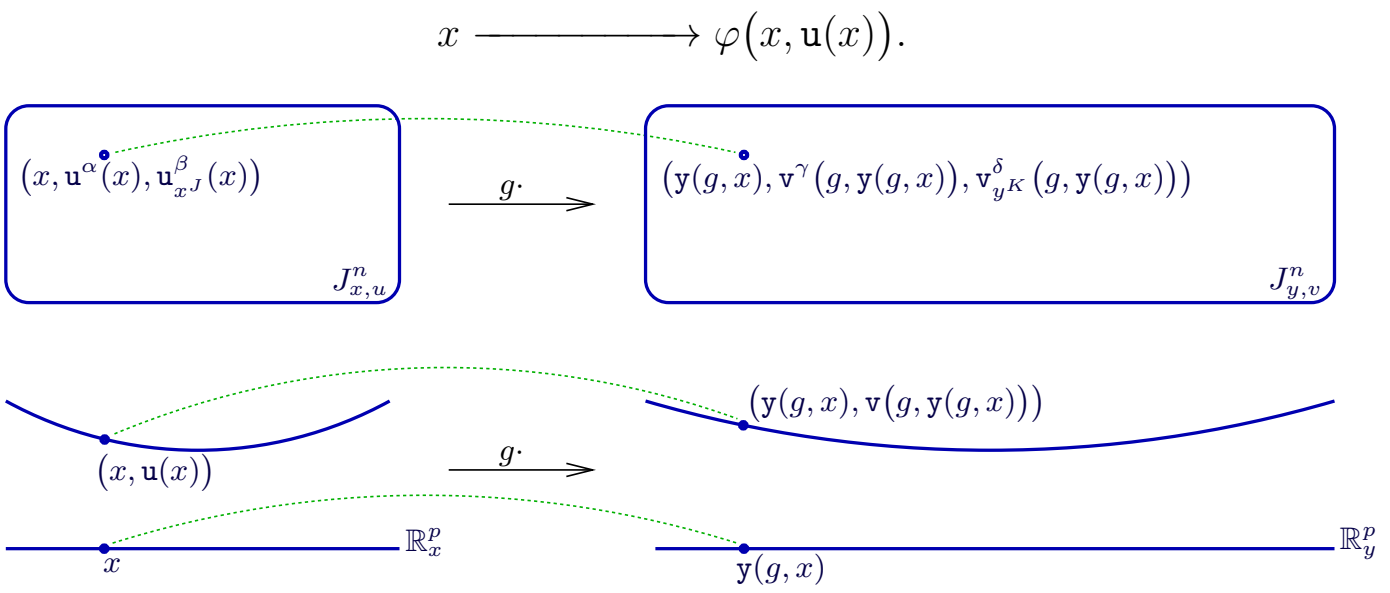

In our case of the action of a local Lie group $G$, we have a family of diffeomorphisms parametrized by $g \in G$, that we can denote as:

$$
(x, u) \longmapsto(\varphi(g, x, u), \psi(g, x, u)) .
$$

On restriction to a graph $\{u=\mathrm{u}(x)\}$, this becomes:

$$
(x, \mathrm{u}(x)) \longmapsto(\varphi(g, x, \mathrm{u}(x)), \psi(g, x, \mathrm{u}(x))) .
$$


Furthermore, as we did in Section 3, we must express how a graph $\{u=\mathrm{u}(x)\}$ is transferred to the target space.

At first, let us abbreviate the family of horizontal diffeomorphisms as:

$$
x \longmapsto \varphi(g, x, \mathrm{u}(x))=: \mathrm{y}(g, x)=: y .
$$

Also, let us denote the family of inverse diffeomorphisms as:

$$
x:=\mathrm{x}(g, y) \longleftarrow y,
$$

by means of certain maps $\mathrm{x}(\mathrm{g}, \cdot)$ coming from an application of the implicit function theorem. Then the source graph $\{u=\mathrm{u}(x)\}$ becomes a family of graphs over the $\mathbb{R}_{y}^{p}$ space:

$$
\begin{aligned}
v & =\psi(g, \mathrm{x}(g, y), \mathrm{u}(\mathrm{x}(g, y))) \\
& =: \mathrm{v}(g, y) .
\end{aligned}
$$

In summary, the action of a local Lie group $G$ on $\mathbb{R}^{p+q}$ provides a family of diffeomorphisms $\phi_{g}(\cdot)=(\varphi(g, \cdot), \psi(g, \cdot))$ together with:

- a $G$-parametrized family of horizontal diffeomorphisms $x \longmapsto \mathrm{y}(g, x)$ from $\mathbb{R}_{x}^{p}$ to $\mathbb{R}_{y}^{p}$;

- a $G$-parametrized family of graphs $\{v=\mathrm{v}(g, y)\}$ in the target space;

which are both associated to any given graph $\{u=\mathrm{u}(x)\}$ in the source space.

Hence as in the figure above, for any $g \in G$, when $x \in \mathbb{R}^{p}$ varies, points $(x, \mathrm{u}(x))$ in a source graph are sent to points $(\mathrm{y}(g, x), \mathrm{v}(g, \mathrm{y}(g, x)))$ in a target graph so that the pointwise one-to-one correspondence between these two graphs reads:

$$
(x, \mathrm{u}(x)) \longleftrightarrow(\mathrm{y}(g, x), \mathrm{v}(g, \mathrm{y}(g, x))) .
$$

We can now express what it really means to be a differential invariant.

Theorem 5.2. A differential invariant $I=I\left(x^{j}, u^{\alpha}, u_{x^{J}}^{\beta}\right)$ takes identical values at points which correspond one to another through the action of any group element $g \in G$ :

$$
I\left(x, \mathrm{u}^{\alpha}(x), \mathrm{u}_{x^{J}}^{\beta}(x)\right) \equiv I\left(\mathrm{y}(g, x), \mathrm{v}^{\gamma}(g, \mathrm{y}(g, x)), \mathrm{v}_{y^{K}}^{\delta}(g, \mathrm{y}(g, x))\right) \quad\left(\forall x \in \mathbb{R}^{p}\right) .
$$

\section{Solving Cross-Section Equations for Curves $C^{1} \subset \mathbb{R}^{2}$ Under $\mathrm{SA}_{2}(\mathbb{R})$}

We illustrate this cross-section approach $([13,33,36])$ in the case of curves $C^{1} \subset \mathbb{R}^{2}$ under the action of the equiaffine group $\mathrm{SA}_{2}(\mathbb{R})$, consisting of area-preserving affine transformations $\mathbb{R}_{x, u}^{1+1} \longrightarrow \mathbb{R}_{y, v}^{1+1}$ :

$$
\begin{aligned}
& y=\mathrm{a} x+\mathrm{b} u+\mathrm{c}, \\
& v=\mathrm{k} x+\mathrm{l} u+\mathrm{m},
\end{aligned} \quad 1=\left|\begin{array}{ll}
\mathrm{a} & \mathrm{b} \\
\mathrm{k} & \mathrm{l}
\end{array}\right| .
$$

According to the preceding sections, any (local) invertible diffeomorphism:

$$
\phi: \quad \mathbb{R}_{x, u}^{2} \longrightarrow \mathbb{R}_{y, v}^{2}
$$

with det Jac $\phi \neq 0$ which is not far from the identity transforms, through an application of the implicit function theorem, sends every graphed curve $\{u=F(x)\}$ into a similar graphed curve $\{y=G(v)\}$. Then tangents are transferred from one curve to the other, 
and higher order jets as well. This means that the diffeomorphism $\phi$ lifts as an invertible transformation between corresponding $n^{\text {th }}$ jet spaces:

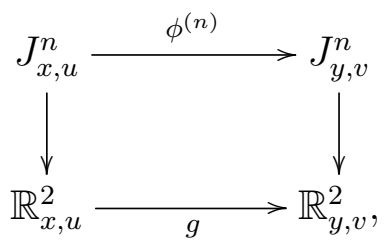

with successive:

$$
\begin{aligned}
\phi^{(1)}:\left(x, u, u_{1}\right) & \longmapsto\left(y(x, u), v(x, u), v_{1}\left(x, u, u_{1}\right)\right), \\
\phi^{(2)}:\left(x, u, u_{1}, u_{2}\right) & \longmapsto\left(y(x, u), v(x, u), v_{1}\left(x, u, u_{1}\right), v_{2}\left(x, u, u_{1}, u_{2}\right)\right),
\end{aligned}
$$

whose first two components are of course those of $\phi$, with certain uniquely determined functions $v_{1}\left(x, u, u_{1}\right), v_{2}\left(x, u, u_{1}, u_{2}\right)$, etc., which use the total differentiation operator:

$$
\mathrm{D}_{x}=\frac{\partial}{\partial x}+u_{1} \frac{\partial}{\partial u}+\sum_{i=1}^{\infty} u_{i+1} \frac{\partial}{\partial u_{i}},
$$

namely:

$$
v_{1}=\frac{\mathrm{D}_{x}(v)}{\mathrm{D}_{x}(y)}, \quad v_{2}=\frac{\mathrm{D}_{x}\left(v_{1}\right)}{\mathrm{D}_{x}(y)}, \quad v_{3}=\frac{\mathrm{D}_{x}\left(v_{2}\right)}{\mathrm{D}_{x}(y)},
$$

However, the expanded formulas are known to rapidly become unwieldy, especially in higher dimensions. At least here with just one independent variable $x$, we have up to jet order $2([21,24])$ :

$$
\begin{aligned}
v_{1}=\frac{v_{x}+u_{1} v_{u}}{y_{x}+u_{1} y_{u}}, & \\
v_{2}=\frac{1}{\left(y_{x}+u_{1} y_{u}\right)^{3}}\left\{u_{2}\left|\begin{array}{ll}
y_{x} & y_{u} \\
v_{x} & v_{u}
\end{array}\right|\right. & +\left|\begin{array}{ll}
y_{x} & y_{x x} \\
v_{x} & v_{x x}
\end{array}\right|+u_{1}\left[2\left|\begin{array}{ll}
y_{x} & y_{x u} \\
v_{x} & v_{x u}
\end{array}\right|-\left|\begin{array}{ll}
y_{x x} & y_{u} \\
v_{x x} & v_{u}
\end{array}\right|\right] \\
& \left.+u_{1}^{2}\left[\left|\begin{array}{ll}
y_{x} & y_{u u} \\
v_{x} & v_{u u}
\end{array}\right|-2\left|\begin{array}{ll}
y_{x u} & y_{u} \\
v_{x u} & v_{u}
\end{array}\right|\right]-u_{1}^{3}\left|\begin{array}{ll}
y_{u u} & y_{u} \\
v_{u u} & v_{u}
\end{array}\right|\right\} .
\end{aligned}
$$

When the diffeomorphism is a special affine transformation:

$$
y=\mathrm{a} x+\mathrm{b} u+\mathrm{c}, \quad v=\mathrm{k} x+\frac{1+\mathrm{b} \mathrm{k}}{\mathrm{a}} u+\mathrm{m},
$$

a computer yields compact formulas:

$$
\begin{aligned}
& v_{1}=\frac{\mathrm{ak}+(1+\mathrm{bk}) u_{1}}{\mathrm{a}\left(\mathrm{a}+\mathrm{b} u_{1}\right)}, \\
& v_{2}=\frac{u_{2}}{\left(\mathrm{a}+\mathrm{b} u_{1}\right)^{3}}, \\
& v_{3}=\frac{-3 \mathrm{~b} u_{2}^{2}+\mathrm{a} u_{3}+\mathrm{b} u_{1} u_{3}}{\left(\mathrm{a}+\mathrm{b} u_{1}\right)^{5}}, \\
& v_{4}=\frac{-10 \mathrm{~b}^{2} u_{1} u_{2} u_{3}-10 \mathrm{ab} u_{2} u_{3}+15 \mathrm{~b}^{2} u_{2}^{3}+2 \mathrm{ab} u_{1} u_{4}+\mathrm{b}^{2} u_{1}^{2} u_{4}+\mathrm{a}^{2} u_{4}}{\left(\mathrm{a}+\mathrm{b} u_{1}\right)^{7}} .
\end{aligned}
$$


The natural cross-section ( $c f$. Section 13):

$$
\left\{y=0, v=0, v_{1}=0, v_{2}=1, v_{3}=0\right\},
$$

enables one to solve the 5 group parameters a, b, c, k, m as:

$$
\begin{aligned}
& \mathrm{a}=\frac{3 u_{2}^{2}-u_{1} u_{3}}{3 u_{2}^{5 / 3}}, \\
& \mathrm{~b}=\frac{u_{3}}{3 u_{2}^{5 / 3}}, \\
& \mathrm{c}=\frac{-3 x u_{2}^{2}+x u_{1} u_{3}-u u_{3}}{3 u_{2}^{5 / 3}}, \\
& \mathrm{k}=-\frac{u_{1}}{u_{2}^{1 / 3}}, \\
& \mathrm{~m}=\frac{-u+x u_{1}}{u_{2}^{1 / 3}}
\end{aligned}
$$

which provides the moving frame map $\rho: J_{x, u}^{n} \longrightarrow \mathrm{SA}_{2}(\mathbb{R})$ for any $n \geqslant 3$, and then, replacing in the formula for $v_{4}$, we obtain what we will call the parabolas invariant:

$$
P:=\frac{1}{3} \frac{-5 u_{3}^{2}+3 u_{2} u_{4}}{u_{2}^{8 / 3}},
$$

and which is also known as the equiaffine curvature.

\section{Equiaffine Group $S A_{3}(\mathbb{R})$ and its Action on Graphed Surfaces $S^{2} \subset \mathbb{R}^{3}$}

Let a source space $\mathbb{R}^{3}$ be equipped with coordinates $(x, y, u)$, and let a target space $\mathbb{R}^{3}$ be equipped with coordinates $(s, t, v)$. We are interested in how local analytic graphed surfaces $\{u=F(x, y)\}$ are mapped to local analytic graphed surfaces $\{v=G(s, t)\}$ through simple transformations $\mathbb{R}_{x, y, u}^{3} \longrightarrow \mathbb{R}_{s, t, v}^{3}$.

7.1. Affine and special affine transformations. The affine group:

$$
A_{3}(\mathbb{R}):=G_{3}(\mathbb{R}) \ltimes \mathbb{R}^{3}
$$

consists of invertible linear transformations in $\mathrm{GL}_{3}(\mathbb{R})$, coupled with translations:

$$
\begin{aligned}
s & =\mathrm{a} x+\mathrm{b} y+\mathrm{c} u+\mathrm{d}, \\
t & =\mathrm{k} x+\mathrm{l} y+\mathrm{m} u+\mathrm{n}, \\
v & =\mathrm{p} x+\mathrm{q} y+\mathrm{r} u+\mathrm{s},
\end{aligned}
$$$$
0 \neq\left|\begin{array}{ccc}
a & b & c \\
k & l & m \\
p & q & r
\end{array}\right|=: \delta .
$$

When the determinant $\delta=1$, so that the volume (and the orientation) of geometric objects remains unchanged, the transformation is called special affine, or equiaffine:

$$
\mathrm{SA}_{3}(\mathbb{R}):=\mathrm{SL}_{3}(\mathbb{R}) \ltimes \mathbb{R}^{3} .
$$

Both groups $\mathrm{A}_{3}(\mathbb{R})$ and $\mathrm{SA}_{3}(\mathbb{R})$ act on surfaces $S^{2} \subset \mathbb{R}^{3}$ graphed as $\{u=F(x, y)\}$.

We write $\mathbb{R}\{x, y\}$ to denote the ring of convergent power series defined in some neighborhood of the origin $(0,0) \in \mathbb{R}^{2}$. Each element $F(x, y) \in \mathbb{R}\{x, y\}$ admits a power series expansion:

$$
F(x, y)=F_{0,0}+F_{1,0} \frac{x^{1}}{1 !}+F_{0,1} \frac{y^{1}}{1 !}+F_{2,0} \frac{x^{2}}{2 !}+F_{1,1} \frac{x^{1} y^{1}}{1 ! 1 !}+F_{0,2} \frac{y^{2}}{2 !}+\cdots,
$$


namely:

$$
F(x, y)=\sum_{k=0}^{\infty} \sum_{l=0}^{\infty} F_{k, l} \frac{x^{k}}{k !} \frac{y^{l}}{l !}
$$

The two monomials $x$ and $y$ generate the maximal ideal $\langle x, y\rangle \subset \mathbb{R}\{x, y\}$ formed of power series with $F(0,0)=0$. For any order $\mathscr{O} \geqslant 0$, the quotient:

$$
\mathbb{R}\{x, y\} /\langle x, y\rangle^{\mathscr{O}+1}
$$

is a free $\mathbb{R}$-module of rank $\left(\begin{array}{c}\mathscr{C}+2 \\ 2\end{array}\right)$, plainly generated by all the monomials $x^{j} y^{k}$ with $j+k \leqslant$ $\mathscr{O}$.

In this article, we will focus on local analytic graphing functions $F(x, y) \in \mathbb{R}\{x, y\}$ of this sort, and on similar functions $G(s, t) \in \mathbb{R}\{s, t\}$, possibly with $F(0,0) \neq 0 \neq G(0,0)$.

Definition 7.2. Two local graphed surfaces $\{u=F(x, y)\}$ and $\{v=G(s, t)\}$ are (special) affinely equivalent if there exists a (special) affine transformation which sends the one to the other - a concrete criterion follows in a second.

We will always consider transformations in $A_{3}(\mathbb{R})$ or in $\mathrm{SA}_{3}(\mathbb{R})$ which are not far from the identity, so that graphs are transformed into graphs. Thus, if we let act a (special) affine transformation $(x, y, u) \longmapsto(s, t, v)$ as above, any point $(x, y, F(x, y))$ is sent to the point $(s, t, G(s, t))$ and we must have:

$$
\mathrm{p} x+\mathrm{q} y+\mathrm{r} u+\mathrm{s}=\left.G(\mathrm{a} x+\mathrm{b} y+\mathrm{c} u+\mathrm{d}, \mathrm{k} x+\mathrm{I} y+\mathrm{m} u+\mathrm{n})\right|_{u=F(x, y)} .
$$

Equivalently, the following fundamental equation:

$$
\mathrm{p} x+\mathrm{q} y+\mathrm{r} F(x, y)+\mathrm{s}=G(\mathrm{a} x+\mathrm{b} y+\mathrm{c} F(x, y)+\mathrm{d}, \mathrm{k} x+\mathrm{l} y+\mathrm{m} F(x, y)+\mathrm{n}),
$$

must hold identically in the domain of convergence of $F(x, y)$.

Question 7.4. How to determine equivalence classes of surfaces $S^{2} \subset \mathbb{R}^{3}$ modulo $\mathrm{A}_{3}(\mathbb{R})$ or $\mathrm{SA}_{3}(\mathbb{R})$ ?

As already presented in a general context, we will see that there exist rational combinations of derivatives of the graphing function $F$ which are invariant under $A_{3}(\mathbb{R})$, or $\mathrm{SA}_{3}(\mathbb{R})$, called differential invariants. We will realize that if $\{u=F(x, y)\}$ is equivalent to $\{v=G(s, t)\}$, differential invariants are 'the same', namely correspond to each other as explained in Section 5 . We will also see that differential invariants determine equivalent classes of (local) surfaces. All this will be clearer later.

7.5. Three types of substitutions. For now, in order to avoid confusion, it is important to differentiate three types of use of the action of a transformation group like $A_{3}(\mathbb{R})$ or $\mathrm{SA}_{3}(\mathbb{R})$.

(S1) Equivalence: Have in hands an explicit (special) affine equivalence between two given surfaces $\{u=F(x, y)\}$ and $\{v=G(s, t)\}$, as above - a situation which rarely occurs $a$ priori.

(S2) Transformation: Start from a given surface $\{u=F(x, y)\}$, apply a (special) affine transformation, and ask what is the equation of the new surface $\{v=G(s, t)\}$ - the answer relying on an application of the implicit function theorem. 
(S3) Normalization: Start from a given surface $\{u=F(x, y)\}$ and ask whether there exist appropriate special affine transformations with put the target graphed surface $\{v=$ $G(s, t)\}$ into a 'simpler', 'normalized', form - the core of the problem, which, after being solved, will enable to know whether two given surfaces are equivalent like in (S1).

In both situations (S2) and (S3), experience tells us that it is more appropriate to consider the inverse transformation $(x, y, u) \longleftarrow(s, t, v)$. We choose to define such an inverse (special) affine transformation using the same letters for group parameters:

$$
\begin{aligned}
& x=\mathrm{a} s+\mathrm{b} t+\mathrm{c} v+\mathrm{d}, \\
& y=\mathrm{k} s+\mathrm{l} t+\mathrm{m} v+\mathrm{n}, \\
& u=\mathrm{p} s+\mathrm{q} t+\mathrm{r} v+\mathrm{s},
\end{aligned}
$$

$$
0 \neq\left|\begin{array}{ccc}
a & b & c \\
k & l & m \\
p & q & r
\end{array}\right|=: \delta \text {. }
$$

For instance, if $F(x, y)$ is given, we can answer (S2) by plugging $(x, y, u)$ in terms of $(s, t, v)$ inside $0=-u+F(x, y)$, which gives:

$$
0=-\mathrm{p} s-\mathrm{q} t-\mathrm{r} v-\mathrm{s}+F(\mathrm{a} s+\mathrm{b} t+\mathrm{c} v+\mathrm{d}, \mathrm{k} s+\mathrm{l} t+\mathrm{m} v+\mathrm{n}),
$$

and then the graphing function $G(s, t)$ of the transformed surface is obtained by solving this equation for $v$, using the implicit function theorem, provided the partial derivative with respect to $v$ is nonvanishing:

$$
0 \neq-\mathrm{r}+\mathrm{c} F_{x}+\mathrm{m} F_{y}
$$

a condition which holds automatically when the transformation is close to the identity, since $\mathrm{r} \approx 1$, while $\mathrm{c} \approx 0 \approx \mathrm{m}$.

Later, we will mainly work in order to perform normalizing substitutions of the sort (S3), first in our treatment of curves done in Sections 13, 16, and then more intensively when studdying parabolic surfaces $S^{2} \subset \mathbb{R}^{3}$, in Sections 17, 18, 19, 20.

\section{Affine Invariancy of the Ranks of Hessian Matrices}

For now, we come back to the simple situation (S1) in which an equivalence is given, not sought for. Differentiating the fundamental equation (7.3) with respect to $x$ and to $y$, we get:

$$
\begin{aligned}
& \mathrm{p}+\mathrm{r} F_{x}=\left(\mathrm{a}+\mathrm{c} F_{x}\right) G_{s}+\left(\mathrm{k}+\mathrm{m} F_{x}\right) G_{t}, \\
& \mathrm{q}+\mathrm{r} F_{y}=\left(\mathrm{b}+\mathrm{c} F_{y}\right) G_{s}+\left(\mathrm{I}+\mathrm{m} F_{y}\right) G_{t} .
\end{aligned}
$$

Therefore, for those transformations of $\mathrm{SA}_{3}(\mathbb{R})$ satisfying:

$$
0 \neq \Lambda:=\left|\begin{array}{ll}
\mathrm{a}+\mathrm{c} F_{x} & \mathrm{k}+\mathrm{m} F_{x} \\
\mathrm{~b}+\mathrm{c} F_{y} & \mathrm{I}+\mathrm{m} F_{y}
\end{array}\right|
$$

a condition which certainly holds for transformations close to the identity, we can solve $G_{s}$ and $G_{t}$ in terms of $F_{x}, F_{y}$ :

$$
G_{s}:=\frac{1}{\Lambda}\left|\begin{array}{cc}
\mathrm{p}+\mathrm{r} F_{x} & \mathrm{k}+\mathrm{m} F_{x} \\
\mathrm{q}+\mathrm{r} F_{y} & \mathrm{I}+\mathrm{m} F_{y}
\end{array}\right| \quad G_{t}=\left|\begin{array}{ll}
\mathrm{a}+\mathrm{c} F_{x} & \mathrm{p}+\mathrm{r} F_{x} \\
\mathrm{~b}+\mathrm{c} F_{y} & \mathrm{q}+\mathrm{r} F_{y}
\end{array}\right| .
$$

Beyond, by keeping differentiating the fundamental equation (7.3), we claim that we can iteratively solve every partial derivative $G_{s^{l} t^{m}}$ in terms of the partial derivatives $\left\{F_{x^{j} y^{k}}\right\}_{j+k \leqslant l+m}$. 
Lemma 8.3. For every order $\mathscr{O} \geqslant 0$, the affine group $\mathrm{A}_{3}(\mathbb{R})$ and the special affine $\mathrm{SA}_{3}(\mathbb{R})$ groups act on Taylor coefficients with $l+m \leqslant \mathscr{O}$ :

$$
G_{l, m}=\text { formula }\left(\begin{array}{lll}
\text { a, b, } & \text { c, d, } \\
\text { k, l, m, } & \text { n, } \\
\text { p, }, \text { q, } & \text { r, } & \text { s, }
\end{array} \quad\left\{F_{j, k}\right\}_{j+k \leqslant l+m}\right) .
$$

Such (complicated) formulas express the induced action of $A_{3}(\mathbb{R})$ or of $S A_{3}(\mathbb{R})$ on the spaces of Taylor coefficients:

$$
\mathbb{R}^{\left(\begin{array}{c}
\mathcal{O}+2 \\
2
\end{array}\right)} \cong \mathbb{R}\{x, y\} /\langle x, y\rangle^{\mathscr{O}+1} .
$$

In the context of jet spaces, Section 3 already showed how to obtain such formulas, provided one restricts considerations to the fiber over the origin $(0,0)$ of the jet space $J_{x, y}^{\mathscr{O}}$. Indeed, remember we introduced the modified total differentiation operators that we can now write after pull-back to the graph $\{u=F(x, y)\}$ as:

$$
\left(\begin{array}{l}
\mathrm{E}_{x} \\
\mathrm{E}_{y}
\end{array}\right)=\left(\begin{array}{cc}
\frac{\mathrm{l}+\mathrm{m} F_{y}}{\Lambda} & \frac{-\mathrm{k}-\mathrm{m} F_{x}}{\Lambda} \\
\frac{-\mathrm{b}-\mathrm{c} F_{y}}{\Lambda} & \frac{\mathrm{a}+\mathrm{c} F_{x}}{\Lambda}
\end{array}\right)\left(\begin{array}{l}
\mathrm{D}_{x} \\
\mathrm{D}_{y}
\end{array}\right),
$$

where $\mathrm{D}_{x}, \mathrm{D}_{y}$ are total differentiation operators, and then, similarly as in Theorem 3.5 but only over the origin $(0,0)$, not over every point $(x, y)-$, we have:

$$
\begin{aligned}
G_{s} & =\mathrm{E}_{x}(\mathrm{p} x+\mathrm{q} y+\mathrm{r} F(x, y)+\mathrm{s}), \\
G_{t} & =\mathrm{E}_{y}(\mathrm{p} x+\mathrm{q} y+\mathrm{r} F(x, y)+\mathrm{s}) .
\end{aligned}
$$

and beyond:

$G_{s s}=\mathrm{E}_{x}\left(G_{s}\right), \quad G_{s t}=\mathrm{E}_{x}\left(G_{t}\right)=\mathrm{E}_{y}\left(G_{s}\right)=G_{t s}, \quad G_{t t}=\mathrm{E}_{y}\left(G_{t}\right)$.

In length, the second-order formulas for Taylor coefficients obtained by differentiating (8.1) once more with respect to $x$ and $y$ read:

$$
\begin{aligned}
\mathrm{r} F_{x x}= & \mathrm{c} F_{x x} G_{s}+\mathrm{m} F_{x x} G_{t}+\left(\mathrm{a}+\mathrm{c} F_{x}\right)^{2} G_{s s}+2\left(\mathrm{a}+\mathrm{c} F_{x}\right)\left(\mathrm{k}+\mathrm{m} F_{x}\right) G_{s t}+\left(\mathrm{k}+\mathrm{m} F_{x}\right)^{2} G_{t t}, \\
\mathrm{r} F_{x y}= & \mathrm{c} F_{x y} G_{s}+\mathrm{m} F_{x y} G_{t} \\
& +\left(\mathrm{a}+\mathrm{c} F_{x}\right)\left(\mathrm{b}+\mathrm{c} F_{y}\right) G_{s s}+\left[\left(\mathrm{a}+\mathrm{c} F_{x}\right)\left(\mathrm{l}+\mathrm{m} F_{y}\right)+\left(\mathrm{b}+\mathrm{c} F_{y}\right)\left(\mathrm{k}+\mathrm{m} F_{x}\right)\right] G_{s t} \\
& +\left(\mathrm{k}+\mathrm{m} F_{x}\right)\left(\mathrm{I}+\mathrm{m} F_{y}\right) G_{t t}, \\
\mathrm{r} F_{y y}= & \mathrm{c} F_{y y} G_{s}+\mathrm{m} F_{y y} G_{t}+\left(\mathrm{b}+\mathrm{c} F_{y}\right)^{2} G_{s s}+2\left(\mathrm{~b}+\mathrm{c} F_{y}\right)\left(\mathrm{l}+\mathrm{m} F_{y}\right) G_{s t}+\left(\mathrm{I}+\mathrm{m} F_{y}\right)^{2} G_{t t} .
\end{aligned}
$$

As is known, the Hessian matrix of a graphed surface $\{u=F(x, y)\}$ is:

$$
\operatorname{Hessian}_{F}=\left(\begin{array}{cc}
F_{x x} & F_{x y} \\
F_{y x} & F_{y y}
\end{array}\right) .
$$

We will denote its determinant by:

$$
H_{F}=\operatorname{det} \operatorname{Hessian}_{F}=F_{x x} F_{y y}-F_{x y}^{2} .
$$

When all entries of the Hessian matrix are identically $0 \equiv F_{x x} \equiv F_{x y} \equiv F_{y y}$, the Taylor series of $F$ reduces to its first order terms $F_{0,0}+F_{1,0} x+F_{0,1} y$, and it is easy to verify the

Proposition 8.4. For a graphed surface $\{u=F(x, y)\}$, the following two properties are equivalent:

(i) there is a special affine transformation $(x, y, u) \longmapsto(s, t, v)$ sending it to a plane $\{v=0\}$; 
(ii) the Hessian matrix Hessian $(F) \equiv\left(\begin{array}{ll}0 & 0 \\ 0 & 0\end{array}\right)$ is identically zero.

The preceding section showed formulas giving $F_{x}, F_{y}, F_{x x}, F_{x y}, F_{y y}$ in terms of $G_{s}, G_{t}$, $G_{s s}, G_{s t}, G_{t t}$. Then a direct check — using a computer! - yields a formula ([26, 5.2]) for the transfer of Hessians determinants:

$$
H_{G}=\text { nonzero } \cdot H_{F},
$$

valid for any (not necessarily special) affine transformation with nonzero quantities:

$$
0 \neq \delta=\left|\begin{array}{ccc}
\mathrm{a} & \mathrm{b} & \mathrm{c} \\
\mathrm{k} & \mathrm{l} & \mathrm{m} \\
\mathrm{p} & \mathrm{q} & \mathrm{r}
\end{array}\right| \quad \quad \quad \quad \quad \quad \quad=\left|\begin{array}{cc}
\mathrm{a}+\mathrm{c} F_{x} & \mathrm{k}+\mathrm{m} F_{x} \\
\mathrm{~b}+\mathrm{c} F_{y} & \mathrm{I}+\mathrm{m} F_{y}
\end{array}\right| \neq 0 .
$$

Theorem 8.5. One has:

$$
G_{s s} G_{t t}-G_{s t}^{2}=\frac{\delta^{2}}{\Lambda^{4}}\left(F_{x x} F_{y y}-F_{x y}^{2}\right)
$$

Consequently, the vanishing or the nonvanishing of the Hessian determinant is an affinely invariant property. In fact, this is a consequence of a more informative

Proposition 8.6. The rank and the signature of the Hessian matrix of a graphed surface $\{u=F(x, y)\}$ are unchanged after any affine transformation. More precisely, under any affine transformation, one has:

$$
\left(\begin{array}{ll}
\mathrm{a}+\mathrm{c} F_{x} & \mathrm{k}+\mathrm{m} F_{x} \\
\mathrm{~b}+\mathrm{c} F_{y} & \mathrm{I}+\mathrm{m} F_{y}
\end{array}\right) \cdot\left(\begin{array}{ll}
G_{s s} & G_{s t} \\
G_{t s} & G_{t t}
\end{array}\right) \cdot\left(\begin{array}{cc}
\mathrm{a}+\mathrm{c} F_{x} & \mathrm{k}+\mathrm{m} F_{x} \\
\mathrm{~b}+\mathrm{c} F_{y} & \mathrm{I}+\mathrm{m} F_{y}
\end{array}\right)^{\mathrm{t}}=\frac{\delta}{\Lambda}\left(\begin{array}{cc}
F_{x x} & F_{x y} \\
F_{y x} & F_{y y}
\end{array}\right) .
$$

Proof. We already wrote above two formulas giving $G_{s}$ and $G_{t}$ in terms of $F_{x}, F_{y}$. Using a computer, starting from the three formulas for second derivatives written above, we may similarly solve $G_{s s}, G_{s t}, G_{t t}$ in terms of $F_{x x}, F_{x y}, F_{y y}, F_{x}, F_{y}$. The formulas are quite large. Equivalently, with $v:=\mathrm{p} x+\mathrm{q} y+\mathrm{r} F(x, y)+\mathrm{s}$ :

$$
G_{s s}=\mathrm{E}_{x}\left(\mathrm{E}_{x}(v)\right), \quad G_{s t}=\mathrm{E}_{x}\left(\mathrm{E}_{y}(v)\right), \quad G_{t t}=\mathrm{E}_{y}\left(\mathrm{E}_{y}(v)\right) .
$$

Still on a computer, we verify that this matrix identity holds.

Definition 8.7. A point $p=\left(x_{p}, y_{p}\right)$ on a graphed surface $\{u=F(x, y)\}$ is called:

- flat if $\operatorname{Hessian}_{F}(p)=\left(\begin{array}{ll}0 & 0 \\ 0 & 0\end{array}\right)$;

- parabolic if $\operatorname{Hessian}_{F}(p)$ has rank 1;

- elliptic if $\operatorname{Hessian}_{F}(p)$ has rank 2 and signature $(2,0)$ or $(0,2)$;

- hyperbolic if $\operatorname{Hessian}_{F}(p)$ has rank 2 and signature $(1,1)$.

Of course, these 4 circumstances are mutually exclusive. After an elementary special affine transformation, we can assume that the graph $u=F(x, y)$ passes through the origin $p=0$ and that $\operatorname{Hessian}_{F}(0)$ is as shown below. 


\begin{tabular}{r|c|l}
\hline$F(x, y)$ & Hessian $_{F}$ at the origin & Type of the origin \\
\hline $\mathrm{O}(3)$ & $\left(\begin{array}{ll}0 & 0 \\
0 & 0\end{array}\right)$ & flat \\
\hline$\frac{1}{2} x^{2}+\mathrm{O}(3)$ & $\left(\begin{array}{ll}1 & 0 \\
0 & 0\end{array}\right)$ & parabolic \\
\hline$\frac{1}{2}\left(x^{2}+y^{2}\right)+\mathrm{O}(3)$ & $\left(\begin{array}{ll}1 & 0 \\
0 & 1\end{array}\right)$ & elliptic \\
\hline$\frac{1}{2}\left(x^{2}-y^{2}\right)+\mathrm{O}(3)$ & $\left(\begin{array}{cc}1 & 0 \\
0 & -1\end{array}\right)$ & hyperbolic \\
\hline
\end{tabular}

The map:

$$
(x, y) \longmapsto \operatorname{rank} \operatorname{Hessian}_{F}(x, y)
$$

is lower semicontinuous, namely, if the Hessian matrix has rank $\tau_{p}$ with $0 \leqslant \tau_{p} \leqslant 2$ at some point $\left(x_{p}, y_{p}\right)$, then at all nearby points $q \sim p$, it has rank $\tau_{q} \geqslant \tau_{p}$. If it has rank 2 at some point, then it has rank 2 in some neighborhood of that point.

If we agree to make rank constancy assumptions, as we will do throughout this article, analytic surfaces $S^{2} \subset \mathbb{R}^{3}$ can be classified as:

- everywhere flat, if the Hessian matrix is identically of rank 0;

- everywhere parabolic, if the Hessian matrix is identically of rank 1;

- everywhere nondegenerate, if the Hessian matrix is everywhere of rank 2.

Nevertheless, it remains in general two kinds of mixed types, where a surface $\{u=$ $F(x, y)\}$ can be:

- flat in a proper closed subset, and parabolic elsewhere, in some dense open subset;

- flat or parabolic in some proper closed subset, and elliptic or hyperbolic elsewhere, in some dense open subset.

In this paper, we will avoid studying mixed types, because it would engage towards singularity theory. Before we focus our attention on everywhere parabolic surfaces, let us briefly review known works about everywhere nondegenerate surfaces $S^{2} \subset \mathbb{R}^{3}$.

\section{Everywhere Elliptic or Hyperbolic Surfaces $S^{2} \subset \mathbb{R}^{3}$ : A Review}

By lower semicontinuity of the rank of a matrix, if the origin is an elliptic (or hyperbolic) point, then there exists a sufficiently small neighborhood which is everywhere elliptic (or hyperbolic).

Theorem 9.1. [40, III, p. 165] Under the action of the equi-affine group $\mathrm{SA}_{3}(\mathbb{R})$, every elliptic surface $S^{2} \subset \mathbb{R}^{3}$ is equivalent to:

$$
u=\frac{1}{2}\left(x^{2}+y^{2}\right)+\frac{C}{6}\left(x^{3}-3 x y^{2}\right)+\mathrm{O}_{x, y}(4),
$$

while every hyperbolic surface is equivalent to one and only of the following three:

$$
\begin{aligned}
& u=\frac{1}{2}\left(x^{2}-y^{2}\right)+\frac{c}{6}\left(x^{3}+3 x y^{2}\right)+\mathrm{O}_{x, y}(4), \\
& u=\frac{1}{2}\left(x^{2}-y^{2}\right)+\frac{C}{6}\left(3 x^{2} y+y^{3}\right)+\mathrm{O}_{x, y}(4), \\
& u=\frac{1}{2}\left(x^{2}-y^{2}\right)+\frac{1}{6}(x+y)^{3}+\mathrm{O}_{x, y}(4),
\end{aligned}
$$

where $C$ is unique up to sign. 
This $C$ is a Taylor coefficient at the origin, but the method of the next Section 12 will show, thanks to the fact that the action of $\mathrm{SA}_{3}(\mathbb{R})$ is (trivially) transitive, that the computation of $C$ for a power series at the origin provides the expression of a corresponding differential invariant at every point $(x, y)$.

Definition 9.2. The quantity:

$$
P:=\frac{1}{2} C^{2}
$$

is a $3^{\text {rd }}$-order equi-affine invariant, called the Pick invariant.

Its explicit expression, at an elliptic point, is:

$$
\begin{aligned}
P=\frac{1}{512} \frac{1}{\left(u_{x x} u_{y y}-u_{x y}^{2}\right)^{11 / 2}} & \left(-18 u_{x x} u_{x x y} u_{x y} u_{x y y} u_{y y}+12 u_{x x x} u_{x y}^{2} u_{x y y} u_{y y}+\right. \\
& +9 u_{x x}^{2} u_{x y y}^{2} u_{y y}+9 u_{x x} u_{x x y}^{2} u_{y y}^{2}-6 u_{x x x} u_{x x y} u_{x y} u_{y y}^{2}- \\
& -6 u_{x x} u_{x x x} u_{x y y} u_{y y}^{2}+u_{x x x}^{2} u_{y y}^{3}+12 u_{x x} u_{x x y} u_{x y}^{2} u_{y y y}- \\
& -8 u_{x x x} u_{x y}^{3} u_{y y y}-6 u_{x x}^{2} u_{x y} u_{x y y} u_{y y y}-6 u_{x x}^{2} u_{x x y} u_{y y} u_{y y y}+ \\
& \left.+6 u_{x x} u_{x x x} u_{x y} u_{y y} u_{y y y}+u_{x x}^{3} u_{y y y}^{2}\right)^{2} .
\end{aligned}
$$

At a hyperbolic point, we replace the factor $\left(u_{x x} u_{y y}-u_{x y}^{2}\right)^{-11 / 2}$ by $\left(u_{x y}^{2}-u_{x x} u_{y y}\right)^{11 / 2}$.

When $C$ is nonzero, we may assume $C>0$. Then the only element in $\mathrm{SA}_{3}(\mathbb{R})$ fixing the standard form above is the identity, hence all the coefficients in the Taylor expansion of $\mathrm{O}_{x, y}(4)$ are also differential invariants.

Under some non-degeneracy conditions, Olver proved in [33] that all those higher order differential invariants can be generated by $C$ and its differentials. Once $C$ is captured in a small neighborhood of the origin, all the differential invariants are known there.

\section{Parabolic Jet Relations}

Take a graph $\{u=F(x, y)\}$, and assume that the Hessian matrix of $F$ :

$$
\operatorname{Hessian}_{F}=\left(\begin{array}{cc}
F_{x x} & F_{x y} \\
F_{y x} & F_{y y}
\end{array}\right)
$$

has rank 1 at every point. After an rotation in the $(x, y)$-space (if necessary), this assumption amounts to:

$$
F_{x x} \neq 0 \equiv F_{x x} F_{y y}-F_{x y}^{2}
$$

Therefore, we can solve:

$$
F_{y y}=\frac{F_{x y}^{2}}{F_{x x}} .
$$

Jet spaces will be equipped with coordinates denoted:

$$
\left(x, y, u, u_{1,0}, u_{0,1}, u_{2,0}, u_{1,1}, u_{0,2}, u_{3,0}, u_{2,1}, u_{1,2}, u_{0,3}, \ldots .\right) .
$$

Sometimes, we will fix an order $\mathscr{O} \geqslant 0$. At first, we have to express all the differential consequences of the resolution $(10.1)$ for $F_{y y}$. 


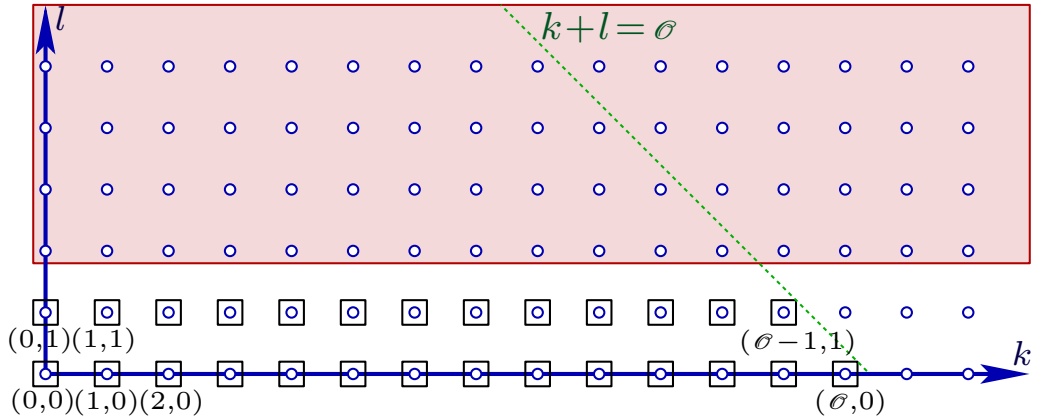

For jets of order 3, we obtain by differentiating with respect to $x$ and to $y$ and by performing replacements:

$$
\begin{aligned}
& F_{x y y}=2 \frac{F_{x y} F_{x x y}}{F_{x x}}-\frac{F_{x y}^{2} F_{x x x}}{F_{x x}^{2}}, \\
& F_{y y y}=3 \frac{F_{x y}^{2} F_{x x y}}{F_{x x}^{2}}-2 \frac{F_{x y}^{3} F_{x x x}}{F_{x x}^{3}},
\end{aligned}
$$

and also for jets of order 4 :

$$
\begin{aligned}
& F_{x x y y}=-4 \frac{F_{x y} F_{x x y} F_{x x x}}{F_{x x}^{2}}+2 \frac{F_{x y} F_{x x x y}}{F_{x x}}+2 \frac{F_{x x y}^{2}}{F_{x x}}+2 \frac{F_{x y}^{2} F_{x x x}^{2}}{F_{x x}^{3}}-\frac{F_{x y}^{2} F_{x x x x}}{F_{x x}^{2}}, \\
& F_{x y y y}=-12 \frac{F_{x y}^{2} F_{x x x} F_{x x y}}{F_{x x}^{3}}+6 \frac{F_{x y} F_{x x y}^{2}}{F_{x x}^{2}}+6 \frac{F_{x y}^{3} F_{x x x}^{2}}{F_{x x}^{4}}+3 \frac{F_{x y}^{2} F_{x x x y}}{F_{x x}^{2}}-2 \frac{F_{x y}^{3} F_{x x x x}}{F_{x x}^{3}}, \\
& F_{y y y y}=-24 \frac{F_{x y}^{3} F_{x x x} F_{x x y}}{F_{x x}^{4}}+12 \frac{F_{x y}^{2} F_{x x y}^{2}}{F_{x x}^{3}}+4 \frac{F_{x y}^{3} F_{x x x y}}{F_{x x}^{3}}+12 \frac{F_{x y}^{4} F_{x x x}^{2}}{F_{x x}^{5}}-3 \frac{F_{x y}^{4} F_{x x x x}}{F_{x x}^{4}} .
\end{aligned}
$$

Similar quite longer formulas exist for $F_{x x x y y}, F_{x x y y y}, F_{x y y y y}, F_{y y y y y}$, for the memory of a computer, this is nothing, even for jets up to order 7 . It is also easy to prove by induction the

Observation 10.2. Every partial derivative $F_{x^{k} y^{l}}$ in the red region with $l \geqslant 2$ expresses rationally in terms of the partial derivatives in the black region:

$$
\left\{F_{x^{k^{\prime}}}\right\}_{k^{\prime} \leqslant k+l}, \quad\left\{F_{x^{k^{\prime \prime}} y}\right\}_{k^{\prime \prime} \leqslant k+l-1},
$$

with denominators containing only powers $\left(F_{x x}\right)^{*}$.

Using the jet notation, this means that we will exclusively work in the submanifold of the jet spaces $J_{x, u}^{\mathscr{O}}$ defined by:

$$
u_{0,2}=\frac{u_{1,1}^{2}}{u_{2,0}},
$$

next:

$$
\begin{aligned}
& u_{1,2}=2 \frac{u_{1,1} u_{2,1}}{u_{2,0}}-\frac{u_{1,1}^{2} u_{3,0}}{u_{2,0}^{2}}, \\
& u_{0,3}=3 \frac{u_{1,1}^{2} u_{2,1}}{u_{2,0}^{2}}-2 \frac{u_{1,1}^{3} u_{3,0}}{u_{2,0}^{3}},
\end{aligned}
$$


further:

$$
\begin{aligned}
& u_{2,2}=2 \frac{u_{2,1}^{2}}{u_{2,0}}-4 \frac{u_{1,1} u_{2,1} u_{3,0}}{u_{2,0}^{2}}+2 \frac{u_{1,1} u_{3,1}}{u_{2,0}}+2 \frac{u_{1,1}^{2} u_{3,0}^{2}}{u_{2,0}^{3}}-\frac{u_{1,1}^{2} u_{4,0}}{u_{2,0}^{2}}, \\
& u_{1,3}=6 \frac{u_{1,1} u_{2,1}^{2}}{u_{2,0}^{2}}-12 \frac{u_{1,1}^{2} u_{3,0} u_{2,1}}{u_{2,0}^{3}}+3 \frac{u_{1,1}^{2} u_{3,1}}{u_{2,0}^{2}}+6 \frac{u_{1,1}^{3} u_{3,0}^{2}}{u_{2,0}^{4}}-2 \frac{u_{1,1}^{3} u_{4,0}}{u_{2,0}^{3}}, \\
& u_{0,4}=12 \frac{u_{1,1}^{2} u_{2,1}^{2}}{u_{2,0}^{3}}-24 \frac{u_{1,1}^{3} u_{2,1} u_{3,0}}{u_{2,0}^{4}}+12 \frac{u_{1,1}^{4} u_{3,0}^{2}}{u_{2,0}^{5}}+4 \frac{u_{1,1}^{3} u_{3,1}}{u_{2,0}^{3}}-3 \frac{u_{1,1}^{4} u_{4,0}}{u_{2,0}^{4}},
\end{aligned}
$$

and so on. Again, this is nothing on a computer.

\section{In Search of a Resolved Cross-Section for Parabolic Surfaces $S^{2} \subset \mathbb{R}^{3}$}

Now, if we let a general affine transformation:

$$
\begin{aligned}
& s=\mathrm{a} x+\mathrm{b} y+\mathrm{c} u+\mathrm{d}, \\
& t=\mathrm{k} x+\mathrm{l} y+\mathrm{m} u+\mathrm{n}, \\
& v=\mathrm{p} x+\mathrm{q} y+\mathrm{r} u+\mathrm{s},
\end{aligned} \quad \delta=\left|\begin{array}{ccc}
\mathrm{a} & \mathrm{b} & \mathrm{c} \\
\mathrm{k} & \mathrm{l} & \mathrm{m} \\
\mathrm{p} & \mathrm{q} & \mathrm{r}
\end{array}\right| \neq 0,
$$

act on graphed surfaces, the first prolongation formulas are:

$$
\begin{aligned}
& v_{1,0}=\frac{\mathrm{lp}-\mathrm{kq}+(\mathrm{lr}-\mathrm{mq}) u_{1,0}+(\mathrm{mp}-\mathrm{kr}) u_{0,1}}{\mathrm{al}-\mathrm{bk}+(\mathrm{cl}-\mathrm{bm}) u_{1,0}+(\mathrm{am}-\mathrm{ck}) u_{0,1}} \\
& v_{0,1}=\frac{\mathrm{aq}-\mathrm{bp}+(\mathrm{cq}-\mathrm{br}) u_{1,0}+(\mathrm{ar}-\mathrm{cp}) u_{0,1}}{\mathrm{al}-\mathrm{bk}+(\mathrm{cl}-\mathrm{bm}) u_{1,0}+(\mathrm{am}-\mathrm{ck}) u_{0,1}},
\end{aligned}
$$

and we of course recognize from (8.2) the denominator:

$$
\Lambda=\mathrm{al}-\mathrm{bk}+(\mathrm{cl}-\mathrm{bm}) u_{1,0}+(\mathrm{am}-\mathrm{ck}) u_{0,1} .
$$

Next, for second-order jets $v_{2,0}, v_{1,1}, v_{0,2}$, we consider only $v_{2,0}, v_{1,1}$, because the jet $v_{0,2}=v_{1,1}^{2} / v_{2,0}$ is dependent. Furthermore, we have to take account of the parabolic jet relations explained in Section 10. Using a computer, we obtain:

$$
\begin{aligned}
& v_{2,0}=\frac{\delta}{\Lambda^{3} u_{2,0}}(\underbrace{\mid u_{2,0}+\mathrm{m} u_{0,1} u_{2,0}-\mathrm{k} u_{1,1}-\mathrm{m} u_{1,0} u_{1,1}}_{=\Pi})^{2}, \\
& v_{1,1}=\frac{\delta}{\Lambda^{3} u_{2,0}} \Pi\left(\mathrm{c} u_{1,0} u_{1,1}+\mathrm{a} u_{1,1}-\mathrm{b} u_{2,0}-\mathrm{c} u_{0,1} u_{2,0}\right),
\end{aligned}
$$

while the formulas for the two independent third-order jets $v_{3,0}$ and $v_{2,1}$ start to become large:

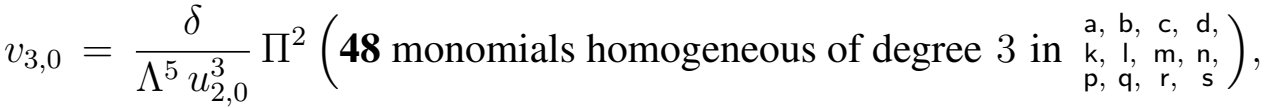

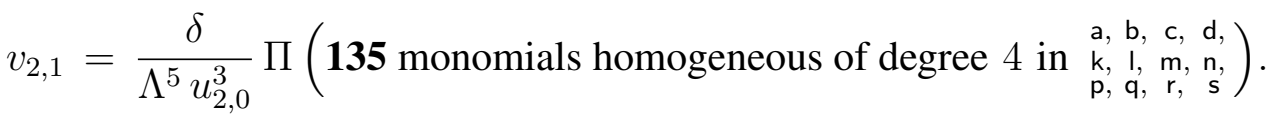

and the last two useful formulas start to become huge:

$$
\begin{aligned}
& v_{4,0} \ni \text { factor in the numerator containing } \mathbf{7 2 0} \text { monomials, } \\
& v_{4,1} \ni \text { factor in the numerator containing } \mathbf{1 4 1 5 6} \text { monomials. }
\end{aligned}
$$




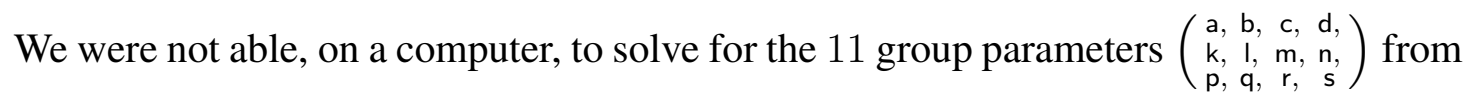
the 11 natural cross-section equations:

$$
\begin{array}{lll}
s=0, \quad t=0, \quad v=0, & v_{1,0}=0, & v_{0,1}=0, \\
& v_{2,0}=1, & v_{1,1}=0, \\
& v_{3,0}=0, & v_{2,1}=1, \\
& v_{4,0}=0, & \\
& & v_{4,1}=0 .
\end{array}
$$

Fortunately, an alternative, more progressive, method works, as we will see in Sections 17, 18, $19,20$.

By solving order-by-order the cross-section equations, they progressively simplify - a lot! - , and we never have to deal with huge expresssions.

\section{The Power Series Method}

Consider a local Lie group $G_{0}$ acting on $\mathbb{R}_{x, u}^{p+q}$, and assume that all of its elements fix the origin $z=0$ :

$$
g_{0} \cdot 0=0
$$

$\left(\forall g_{0} \in G_{0}\right)$.

As usual, we denote coordinates on the target space $\mathbb{R}_{y, v}^{p+q}$ as $w=(y, v)$. For the moment, we do not necessarily assume that $G_{0}$ is the isotropy subgroup of the origin for the action of a certain larger group $G \supset G_{0}$.

When working with power series, we will abandon the notation $J=j_{1}, \ldots, j_{\lambda}$ used for jet spaces in the preceding sections, with $1 \leqslant j_{1}, \ldots, j_{\lambda} \leqslant p$ not recording repetitions, and instead, we will employ the standard multi-index notation — with the same letter(s) -

$$
J=\left(j_{1}, \ldots, j_{p}\right) \in \mathbb{N}^{p}
$$

The advantage is that we can introduce useful quantities which would be otherwise difficult to denote:

$$
J !:=j_{1} ! \cdots j_{p} ! \quad \text { and } \quad|J|:=j_{1}+\cdots+j_{p},
$$

and also:

$$
x^{J}:=x^{j_{1}} \cdots x_{p}^{j_{p}} .
$$

As before, we denote the $G_{0}$-action by $w=g_{0} \cdot z$, or equivalently:

$$
y=y\left(g_{0}, x, u\right), \quad v=v\left(g_{0}, x, u\right) .
$$

Now, consider a (converging) power series mapping $\mathbb{R}^{p} \ni x \longmapsto F(x) \in \mathbb{R}^{q}$ in the source space which vanishes at the origin:

$$
u^{\alpha}=\sum_{|J| \geqslant 1} F_{J}^{\alpha} \frac{x^{J}}{J !} .
$$

Every diffeomorphism $z \longmapsto g_{0} \cdot z=$ : $w$ corresponding to a group parameter $g_{0} \in G_{0}$ close to the identity element then transforms the graph $\{u=F(x)\}$ of this power series 
into another graph $\left\{v=G\left(g_{0}, y\right)\right\}$ depeding on $g_{0}$, still passing through the origin $(y, v)=$ $(0,0)$, whose graphing function also has a power series expansion:

$$
v^{\beta}:=\sum_{|K| \geqslant 1} G_{K}^{\beta} \frac{y^{\beta}}{K !},
$$

with coefficients:

$$
G_{K}^{\beta}=G_{K}^{\beta}\left(g_{0},\left\{F_{L}^{\gamma}\right\}_{1 \leqslant|L| \leqslant|K|}^{1 \leqslant \gamma \leqslant q}\right),
$$

depending on the coefficients of the source power series, and on the group parameters as well - of course. One easily convinces oneself that, as is written, the $G_{K}^{\beta}$ only depend on power series coefficients $F_{J}^{\alpha}$ of order $|J| \leqslant|K|$.

Definition 12.1. A power series invariant of order $n \geqslant 1$ is a function of the Taylor coefficients:

$$
I=I\left(\left\{F_{J}^{\alpha}\right\}_{1 \leqslant|J| \leqslant n}^{1 \leqslant \alpha \leqslant q}\right)
$$

which has unchanged value after the action of any element in our local Lie group:

$$
I\left(\left\{G_{K}^{\beta}\left(g_{0},\left\{F_{L}^{\gamma}\right\}_{1 \leqslant|L| \leqslant|K|}^{1 \leqslant \gamma \leqslant q}\right)\right\}_{1 \leqslant|K| \leqslant n}^{1 \leqslant \beta \leqslant q}\right)=I\left(\left\{F_{J}^{\alpha}\right\}_{1 \leqslant|J| \leqslant n}^{1 \leqslant \alpha \leqslant q}\right) . \quad\left(\forall g_{0} \in G_{0}\right) .
$$

This concept has a meaning only at the origin! It only concerns the derivatives at 0 of the graphing functions $\mathrm{u}^{\alpha}(x)$ :

$$
\left\{u_{x^{J}}^{\alpha}(0)\right\}_{J \in \mathbb{N}_{*}^{p}}^{1 \leqslant \alpha \leqslant q}
$$

By contrast, the general theory of differential invariants is able to handle derivatives at all points in the source horizontal space:

$$
\left\{\mathrm{u}_{x^{J}}^{\alpha}(x)\right\}_{J \in \mathbb{N}_{*}^{p}, x \text { varies in } \mathbb{R}^{p}}^{1 \leqslant \alpha \leqslant q}
$$

So it seems that this notion of power series invariant is quite restrictive! But a bit paradoxically — and quite the contrary! - , we will rapidly realize that the power series invariants do capture all differential invariants at any point $x \in \mathbb{R}^{p}$, provided only that the larger group $G \supset G_{0}=\operatorname{Iso}(G, 0)$ contains the ambient translations.

In [36], it is shown how:

$$
\text { invariants in jet spaces } \leadsto \text { power series invariants. }
$$

Our goal now is to explore the reverse transmission:

$$
\text { invariants in jet spaces } \sim \text { power series invariants, }
$$

which will bring some computational advantages.

Taking a local Lie group $G$ acting on a neighborhood of $0 \in \mathbb{R}_{x, u}^{p+q}$, not necessarily fixing the origin, we will make two kinds of assumptions.

Hypothesis 12.2. The group $G$ contains all translations of the ambient space:

$$
\left(x^{i}, u^{\alpha}\right) \longmapsto\left(x^{i}+\mathrm{a}^{i}, u^{\alpha}+\mathrm{b}^{\alpha}\right),
$$

whence $\operatorname{dim} G \geqslant p+q$.

Equivalently, the Lie algebra $\mathfrak{g}=\operatorname{Lie}(G)$ of its action contains all the unit coordinate infinitesimal generators:

$$
\partial_{x^{i}}, \quad \partial_{u^{\alpha}}
$$

The proof of the following result is elementary. 
Theorem 12.3. If $G$ contains all ambient translations, then for any jet order $n \geqslant 0$, all differential invariants of $G$ are independent of $x^{i}, u^{\alpha}$ :

$$
I=I\left(\left\{u_{x^{J}}^{\beta}\right\}_{1 \leqslant|J| \leqslant n}^{1 \leqslant \beta \leqslant q}\right)
$$

and there is a one-to-one correspondence:

$$
\text { Differential invariants of } G \longleftrightarrow \text { Power series invariants of } G \text {. }
$$

More precisely — and this is the interesting aspect! - , the theorem tells us that starting from a power series invariant determined in a way that will be explained later on:

$$
I=I\left(\left\{F_{J}^{\alpha}\right\}_{2 \leqslant|J| \leqslant n}^{1 \leqslant \alpha \leqslant q}\right)=I\left(\left\{u_{x^{J}}^{\alpha}(0)\right\}_{2 \leqslant|J| \leqslant n}^{1 \leqslant \alpha \leqslant q}\right),
$$

one deduces instantly a differential invariant by just replacing the origin with any horizontal $x \in \mathbb{R}^{p}$ :

$$
I=I\left(\left\{\mathrm{u}_{x^{J}}^{\alpha}(x)\right\}_{2 \leqslant|J| \leqslant n}^{1 \leqslant \alpha \leqslant q}\right),
$$

and in particular, at the origin, one recovers the starting power series invariant.

Example 12.4. Consider the action of the special Euclidean group $\mathrm{SE}_{2}(\mathbb{R}):=\mathrm{SO}_{2}(\mathbb{R}) \ltimes \mathbb{R}^{2}$ on curves $\{u=F(x)\}$ in $\mathbb{R}_{x, u}^{2}$. Because this action is transitive, we can restrict our attention to curves passing through $(0,0)$ and to rotations:

$$
\begin{aligned}
& x=\cos \theta y-\sin \theta v, \\
& u=\sin \theta y+\cos \theta v \text {, } \\
& \text { abbreviated as } \\
& \begin{array}{l}
x=\mathrm{c} y-\mathrm{s} v, \\
u=\mathrm{s} y+\mathrm{c} v,
\end{array}
\end{aligned}
$$

with $s^{2}+c^{2}=1$. When we deal with the local Lie group near the identity $(s, c)=(0,1)$, we may assume $c>0$. Two graphed power series:

$u=F(x)=F_{1} \frac{x^{1}}{1 !}+F_{2} \frac{x^{2}}{2 !}+\cdots \quad$ and $\quad v=G(y)=G_{1} \frac{y^{1}}{1 !}+G_{2} \frac{y^{2}}{2 !}+\cdots$

are mapped one to another if and only if:

$$
0 \equiv-\mathrm{s} y-\mathrm{c} G(y)+F(\mathrm{c} y-\mathrm{s} G(y)) \quad \text { (in } \mathbb{C}\{y\}) .
$$

In this identity, the first and second order terms read:

$0 \equiv\left(-\mathrm{s}-\mathrm{c} G_{1}+\mathrm{c} F_{1}-\mathrm{s} F_{1} G_{1}\right) \frac{y^{1}}{1 !}+\left(-\mathrm{c} G_{2}-\mathrm{s} F_{1} G_{2}+\mathrm{c}^{2} F_{2}-2 \mathrm{cs} F_{2} G_{1}+\mathrm{s}^{2} F_{2} G_{1}^{2}\right) \frac{y^{2}}{2 !}+\mathrm{O}_{y}(3)$.

We can make $G_{1}:=0$ thanks to the choice of $\theta \in \mathbb{R}$ satisfying:

$$
0=-\sin \theta+\cos \theta F_{1} \quad \Longleftrightarrow \quad \frac{\mathrm{s}}{\mathrm{c}}=F_{1} .
$$

Next, we may verify that the transformation of $\mathrm{SO}_{2}(\mathbb{R})$ with $c>0$ which stabilizes this normalization of first order terms, namely sends:

$$
u=0+F_{2} \frac{x^{2}}{2 !}+\cdots \quad \text { to } \quad v=0+G_{2} \frac{y^{2}}{2 !}+\cdots,
$$

is the identity.

Looking at the second order term above, with $G_{1}=0$, we get:

$$
G_{2}=F_{2} \frac{c}{1+\frac{s}{c} F_{1}}=F_{2} \frac{c}{1+F_{1}^{2}},
$$


and using $1=\mathrm{s}^{2}+\mathrm{c}^{2}=\mathrm{c}^{2} F_{1}^{2}+\mathrm{c}^{2}$, which gives $\mathrm{c}=\frac{1}{\sqrt{1+F_{1}^{2}}}$, we conclude that we have obtained a power series invariant:

$$
v_{y y}(0)=u_{x x}(0) \frac{1}{\left(1+u_{x}(0)^{2}\right)^{3 / 2}},
$$

which yields a differential invariant at any point $x$ :

$$
u_{x x}(x) \frac{1}{\left(1+u_{x}(x)^{2}\right)^{3 / 2}},
$$

We have thus recovered the Euclidean curvature of curves in the plane by applying the power series method. This method also works for the full Euclidean group $\mathrm{SE}_{2}(\mathbb{R})$, not just the local one. A similar calculation for $c \in[-1,1]$ instead of $c>0$ shows that there are two normal forms: $v=0+G_{2} \frac{y^{2}}{2}+\cdots$ and $v=0-G_{2} \frac{y^{2}}{2}-\cdots$, for every nonlinear curve. These two forms are equivalent to each other by a semi-circle rotation $(s, c)=(0,1)$. So our power series invariant for the full group of order 2 is $\left|G_{2}\right|=\frac{\left|F_{2}\right|}{\left(1+F_{1}^{2}\right)^{3 / 2}}$, which yields a differential invariant at any point $x$ :

$$
\left|u_{x x}(x)\right| \frac{1}{\left(1+u_{x}(x)^{2}\right)^{3 / 2}} .
$$

Beyond this (too) simple example, we will see how the method works in many other contexts.

Furthermore, the interesting reverse transmission:

$$
\text { invariants in jet spaces } \sim \text { power series invariants, }
$$

holds in other situation, as is expressed by our second

Hypothesis 12.5. The group $G$ contains all translations of the ambient space, and all vertical transvections:

$$
\left(x^{i}, u^{\alpha}\right) \longmapsto\left(x^{i}, u^{\alpha}+\sum_{1 \leqslant i \leqslant p} \mathrm{c}_{i}^{\alpha} x^{i}\right),
$$

whence $\operatorname{dim} G \geqslant p+q+p q$.

Equivalently, the Lie algebra $\mathfrak{g}=\operatorname{Lie}(G)$ contains all the infinitesimal generators:

$$
\partial_{x^{i}}, \quad \partial_{u^{\alpha}}, \quad x^{j} \partial_{u^{\beta}} .
$$

Theorem 12.6. If $G$ contains all ambient translations and all vertical transvections, then for any jet order $n \geqslant 0$, all differential invariants of $G$ are independent of $x^{i}, u^{\alpha}, u_{x^{j}}^{\beta}$ :

$$
I=I\left(\left\{u_{x^{J}}^{\gamma}\right\}_{2 \leqslant|J| \leqslant n}^{1 \leqslant \gamma \leqslant q}\right)
$$

and there is a one-to-one correspondence:

$$
\text { Differential invariants of } G \longleftrightarrow \text { Power series invariants of } G \text {. }
$$

More precisely, the concerned power series invariants can be searched for power series starting only at order 2 :

$$
u^{\alpha}=\sum_{|J| \geqslant 2} F_{J}^{\alpha} \frac{x^{J}}{J !} \quad \text { and } \quad v^{\beta}=\sum_{|K| \geqslant 2} G_{K}^{\beta} \frac{y^{K}}{K !} .
$$


At least three aspects of the power series method are attractive.

- Prolongations of a $G_{0}$-action to jet spaces of any order $n \geqslant 0$ can be performed 'automatically', especially on a computer, for there is no need to write down the complicated formulas $\mathrm{E}_{k}\left(v^{\alpha}\right)$ of Theorem 3.5 , they are implicitly performed after replacement of $y=y\left(g_{0}, x, u\right)$ and $v=v\left(g_{0}, x, u\right)$ inside $0=-v+G(y)$.

- The search for power series invariant takes place above only 1 point, the origin $0 \in \mathbb{R}^{m}$, no differentiations are required anymore, just computations with Taylor coefficients, which are constants, that is to say, the - nonlinear! - action of $G_{0}$ is considered just on a vector space, not on a (jet) bundle.

- In presence of differential relations, as will be illustrated in Sections 17, 18, 19, 20, such differential relations can also be 'automatically' implemented on Taylor series, and this saves computation time.

Lastly, there is another advantage of the power series method.

- A progressive stratification of the normalisations of group parameters for increasing fixed orders $|J|=1,2,3, \ldots$ conducts to a high proximity with the Cartan method of equivalence, and its famous reductions and prolongations of $G$-structures.

To explain these claims, let us exhibit another elementary example, that of curves in the plane $\mathbb{R}_{x, u}^{1+1}$ under the special affine group.

\section{Special Affine Power Series Invariants of Curves in $\mathbb{R}^{2}$}

In $\mathbb{R}^{2} \ni(x, u)$, we consider a graphed curve passing through the origin normalized to order 1:

$$
0=-u+F(x)=-u+F_{2} \frac{x^{2}}{2 !}+F_{3} \frac{x^{3}}{3 !}+\cdots
$$

which satisfies $F_{2} \neq 0$. These formal coefficients $F_{i}$ will be re-initialized in later stages of the process. But at the beginning, we assign these Taylor coefficients to be initial functional jets:

$$
F_{i}:=u_{i}=u_{i}(x),
$$

corresponding to $i^{\text {th }}$ derivatives $u_{x^{i}}(x)$ at arbitrary points $(x, u(x))$ of the curve, as was explained in Section 12. All power series computations we will perform at the origin will therefore have an interesting meaning at every point of any curve in $\mathbb{R}^{2}$ : such is the 'power' of power series!

13.1. First loop. The special affine linear group $S_{2}(\mathbb{R})$ consists of matrices $\left(\begin{array}{l}a \\ c\end{array}\right)$ having determinant $1=\mathrm{ad}-\mathrm{bc}$. Without primes or bars, target coordinates will be denoted $(y, v)$.

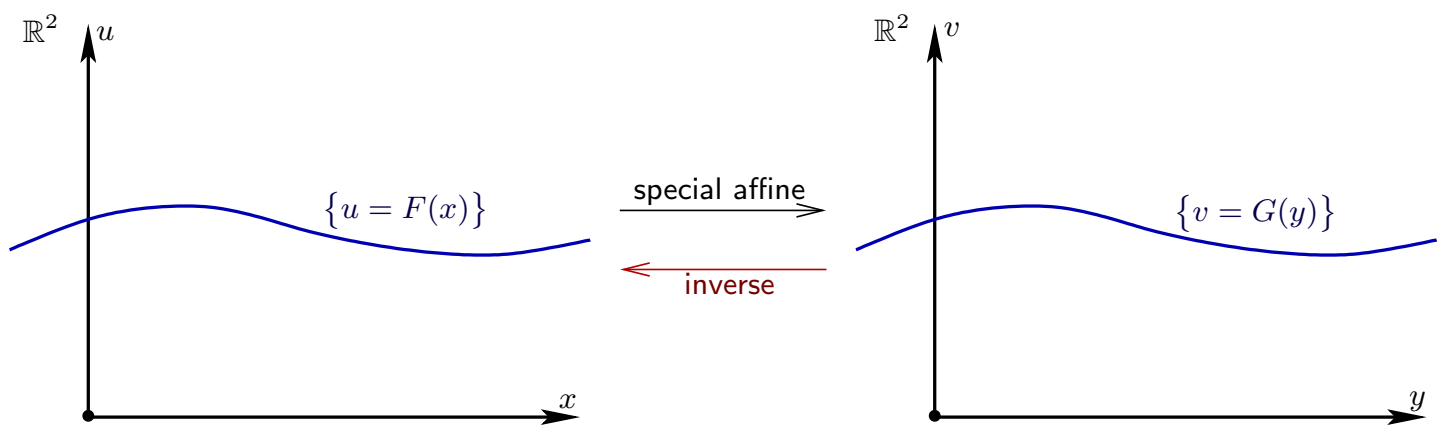


We consider special affine transformations:

$$
\mathbb{R}_{x, u}^{2} \longrightarrow \mathbb{R}_{y, v}^{2}
$$

which are not too far from the identity, so that any analytic graph $\{u=F(x)\}$ is sent to a similar graphed curve $\{v=G(y)\}$.

Since the source power series $F(x)=\sum_{i \geqslant 2} F_{2} \frac{x^{i}}{i !}$ is given and since we want to simplify the target power series $G(y)=\sum_{j \geqslant 2} G_{j} \frac{y^{j}}{j !}$, it is more natural to work with the inverse special affine transformation, which is also represented by means of an $\mathrm{SL}_{2}(\mathbb{R})$ matrix as:

$$
x=\mathrm{a} y+\mathrm{b} v, \quad u=\mathrm{c} y+\mathrm{d} v,
$$

Then the graphing function $G(y)$ is uniquely determined, by a fundamental equation:

$$
\left.0 \equiv-(\mathrm{c} y+\mathrm{d} v)+\left.F(\mathrm{a} y+\mathrm{b} v)\right|_{\text {replace } v=G(y)} \quad \text { (in } \mathbb{R}\{y\}\right),
$$

holding identically as a power series of the single horizontal variable $y$ - why and how $G$ is thusly determined will be clear in a while. The game is to use the group parameters freedom a, b, c, d in order to 'kill' as much as possible coefficients $G_{j}$.

After an affine transformation, we may of course assume that our target graph enjoys a similar first-order normalization $v=\mathrm{O}_{y}(2)$, namely:

$$
0=-v+G(y)=-v+G_{2} \frac{y^{2}}{2 !}+G_{2} \frac{y^{3}}{3 !}+\cdots .
$$

Then performing the plain replacement above:

$$
0 \equiv-\mathrm{c} y-\mathrm{d} G(y)+F(\mathrm{a} y+\mathrm{b} G(y)),
$$

we glean first-order terms which must vanish:

$$
0 \equiv-\mathrm{c} y+\mathrm{O}_{y}(2)
$$

(in $\mathbb{R}\{y\}$ ).

Lemma 13.3. The subgroup of $\mathrm{SL}_{2}(\mathbb{R})$ sending $v=\mathrm{O}_{y}(2)$ to $u=\mathrm{O}_{x}(2)$ is 2-dimensional and consists of matrices:

$$
G_{\text {stab }}^{(1)}: \quad\left(\begin{array}{cc}
a & b \\
0 & \frac{1}{a}
\end{array}\right) \quad(a \neq 0) .
$$

Thus, we have computed the subgroup which stabilizes the current normal form of our power series. In later stages of the process, higher order jets stabilizer subgroups:

$$
\mathrm{SL}_{2}(\mathbb{R}) \supset \mathrm{G}_{\text {stab }}^{(1)} \supset \mathrm{G}_{\text {stab }}^{(2)} \supset \mathrm{G}_{\text {stab }}^{(3)} \supset \cdots \supset \mathrm{G}_{\text {stab }}^{(\tau)}=\{e\}
$$

will naturally appear until final reduction to identity. A deep proximity exists with Cartan's method of equivalence.

But before jumping to the second loop of the 'algorithm', we must examine how this reduced subgroup $\mathrm{G}_{\text {stab }}^{(1)}$ acts on the second order term $y^{2}$ in 13.2 , getting:

$$
0 \equiv\left(-\frac{1}{a} G_{2}+a^{2} F_{2}\right) \frac{y^{2}}{2 !}+\mathrm{O}_{y}(3) .
$$

From our assumption $F_{2} \neq 0$, we deduce that $G_{2} \neq 0$ is inherited.

Furthermore, taking a $:=\frac{1}{F_{2}^{1 / 3}}$, we can make $G_{2}:=1$ by means of the specific matrix we choose $b:=0$ for simplicity - :

$$
\left(\begin{array}{cc}
\frac{1}{F_{2}^{1 / 3}} & 0 \\
0 & F_{2}^{1 / 3}
\end{array}\right) \in \mathrm{G}_{\mathrm{stab}}^{(1)} .
$$


Then with this precise special affine transformation, a computation of the higher order terms in (13.2) gives us:

$$
0 \equiv\left(-F_{2}^{1 / 3} G_{3}+\frac{F_{3}}{F_{2}}\right) \frac{y^{3}}{3 !}+\left(-F_{2}^{1 / 3} G_{4}+\frac{F_{4}}{F_{2}^{4 / 3}}\right) \frac{y^{4}}{4 !}+\left(-F_{2}^{1 / 3} G_{5}+\frac{F_{5}}{F_{2}^{5 / 3}}\right) \frac{y^{5}}{5 !}+\cdots,
$$

and we obtain as promised unique determinations:

$$
G_{i}:=\frac{F_{i}}{F_{2}^{\frac{1+i}{3}}}
$$

Finally, remembering that the $F_{i}$ were formal variables representing the functional jets $u_{x^{i}}(x)=u_{i}(x)=u_{i}=F_{i}$, we see that the coefficients of the transformed curve $\{v=$ $G(y)\}$ have become:

$$
G_{2}:=1, \quad G_{i}:=\frac{u_{i}}{u_{2}^{\frac{1+i}{3}}}
$$

13.4. Second loop. In order to avoid indices heaviness, we keep the same notation $\{u=$ $F(x)\}$ and $\{v=G(y)\}$, which means that what we now call $F$ is the $G$ of the end of the previous loop. So in terms of the initial functional jets $u_{i}$, we have in fact re-assigned:

$$
F_{2}:=1, \quad F_{i}:=\frac{u_{i}}{u_{2}^{\frac{1+i}{3}}}
$$

Keeping this in memory, we will now work formally with power series coefficients $F_{i}$ and $G_{j}$, and only at the end of the current loop will we express the result in terms of these $F_{i}=u_{i} / u_{2}^{(1+i) / 3}$.

So both our source and target graphed curves may be assumed to have terms normalized up to order 2 included:

$$
u=\frac{x^{2}}{2 !}+F_{3} \frac{x^{3}}{3 !}+\cdots \quad \text { and } \quad v=\frac{y^{2}}{2 !}+G_{3} \frac{y^{3}}{3 !}+\cdots .
$$

Remembering that Lemma 13.3 already showed that stabilization up to order 1 forces $c=0$, in order to determine the subgroup $\mathrm{G}_{\mathrm{stab}}^{(2)} \subset \mathrm{SL}_{2}(\mathbb{R})$, we can work within $\mathrm{G}_{\mathrm{stab}}^{(1)}$.

Lemma 13.5. The subgroup of $\mathrm{SL}_{2}(\mathbb{R})$ sending $u=\frac{1}{2} x^{2}+\mathrm{O}_{x}(3)$ to $v=\frac{1}{2} y^{2}+\mathrm{O}_{y}(3)$ is 1-dimensional and consists of matrices:

$$
G_{\text {stab }}^{(2)}: \quad\left(\begin{array}{ll}
1 & \mathrm{~b} \\
0 & 1
\end{array}\right)
$$

Proof. Hence with $\mathrm{c}=0$, back to 13.2 , we get $\mathrm{a}^{3}=1$ from:

$$
0 \equiv\left(-\frac{1}{a}+a^{2}\right) \frac{y^{2}}{2 !}+\mathrm{O}_{y}(3)
$$

Thus, as promised, we have determined the subgroup $G_{\text {stab }}^{(2)}$ which stabilizes the current normal form of our power series, and as we will see soon, the process will stop at stage 3:

$$
\mathrm{SL}_{2}(\mathbb{R}) \supset \mathrm{G}_{\text {stab }}^{(1)} \supset \mathrm{G}_{\text {stab }}^{(2)} \supset \mathrm{G}_{\text {stab }}^{(3)}=\{e\} .
$$

For the time being, let us examine how this reduced group $\mathrm{G}_{\mathrm{stab}}^{(2)}$ acts on the third order term $y^{3}$ in 13.2):

$$
0 \equiv\left(-G_{3}+F_{3}+3 \mathrm{~b}\right) \frac{y^{3}}{3 !}+\mathrm{O}_{y}(4)
$$


We can make $G_{3}=0$ with b $:=-\frac{1}{3} F_{3}$, by means of the specific matrix:

$$
\left(\begin{array}{cc}
1 & -F_{3} / 3 \\
0 & 1
\end{array}\right)
$$

Then with this precise special affine transformation, higher order vanishing terms:

$$
0 \equiv\left(-G_{4}-\frac{5}{3} F_{3}^{2}+F_{4}\right) \frac{y^{4}}{4 !}+\left(-G_{5}-\frac{5}{3} F_{3} G_{4}+F_{5}+\frac{5}{3} F_{3}^{2}-\frac{10}{3} F_{3} F_{4}\right) \frac{y^{5}}{5 !}+\mathrm{O}_{y}(6)
$$

conduct us to:

$$
G_{4}:=-\frac{5}{3} F_{3}^{2}+F_{4}, \quad G_{5}:=F_{5}+\frac{40}{9} F_{3}^{3}-5 F_{3} F_{4} .
$$

Coming back to the functional jets, we obtain:

$G_{2}:=1, \quad G_{3}:=0, \quad G_{4}:=\frac{1}{3} \frac{-5 u_{3}^{2}+3 u_{2} u_{4}}{u_{2}^{8 / 3}}, \quad G_{5}:=\frac{1}{9} \frac{9 u_{2}^{2} u_{5}-45 u_{2} u_{3} u_{4}+40 u_{3}^{3}}{u_{2}^{4}}$.

13.6. Third loop. We start by re-assigning:

$F_{2}:=1, \quad F_{3}:=0, \quad F_{4}:=\frac{1}{3} \frac{-5 u_{3}^{2}+3 u_{2} u_{4}}{u_{2}^{8 / 3}}, \quad F_{5}:=\frac{1}{9} \frac{9 u_{2}^{2} u_{5}-45 u_{2} u_{3} u_{4}+40 u_{3}^{3}}{u_{2}^{4}}$.

We again work with formal $u=\sum F_{i} \frac{x^{i}}{i}$ and $v=\sum G_{j} \frac{y^{j}}{j !}$ assuming the normalizations:

$$
F_{2}=1, \quad F_{3}=0 \quad \text { and } \quad G_{2}=1, \quad G_{3}=0 .
$$

Naturally, since only 1 degree of freedom was left at the previous stage, the condition that the coefficient 0 of $\frac{x^{3}}{3 !}$ is left unchanged drops the group dimension by 1 , and it is easy to verify the

Lemma 13.7. The subgroup of $\mathrm{SL}_{2}(\mathbb{R})$ sending $v=\frac{y^{2}}{2}+0+\mathrm{O}_{y}(4)$ to $u=\frac{x^{2}}{2}+0+\mathrm{O}_{x}(4)$ is 0-dimensional and reduces to the identity:

$$
G_{\text {stab }}^{(3)}: \quad\left(\begin{array}{ll}
1 & 0 \\
0 & 1
\end{array}\right)
$$

The algorithm therefore stops, and a first result, valid only at the level of power series at the origin, is a corollary of this reduction to an $\{e\}$-group.

Theorem 13.8. (1) Given a real analytic curve $\{u=F(x)\}$ in $\mathbb{R}^{2}$ passing through the origin which satisfies:

$$
F_{x x}(0) \neq 0,
$$

there always exists an $\mathrm{SL}_{2}(\mathbb{R})$ transformation which puts it into the form

$$
u=\frac{x^{2}}{2 !}+0+F_{4} \frac{x^{4}}{4 !}+F_{5} \frac{x^{5}}{5 !}+\sum_{i \geqslant 6} F_{i} \frac{x^{i}}{i !} .
$$

(2) Any other such real analytic curve $\{v=G(y)\}$ similarly put into the form:

$$
v=\frac{y^{2}}{2 !}+0+G_{4} \frac{y^{4}}{4 !}+G_{5} \frac{y^{5}}{5 !}+\sum_{j \geqslant 6} G_{j} \frac{y^{j}}{j !},
$$

is $\mathrm{SL}_{2}(\mathbb{R})$-equivalent to $\{u=F(x)\}$ above if and only if all Taylor coefficients match:

$$
G_{4}=F_{4}, \quad G_{5}=F_{5}, \quad G_{i}=F_{i} \quad(\forall i \geqslant 6) .
$$


As explained in Section 12, the power series coefficients so obtained $F_{4}, F_{5}$, are differential invariants at any point $(x, u(x))$ of the curve, and beyond, the explicit expressions of the next two are:

$$
\begin{aligned}
& F_{6}:=\frac{1}{9} \frac{9 u_{2}^{3} u_{6}-63 u_{2}^{2} u_{3} u_{5}+105 u_{2} u_{3}^{2} u_{4}-35 u_{3}^{4}}{u_{2}^{16 / 3}}, \\
& F_{7}:=\frac{1}{9} \frac{9 u_{2}^{4} u_{7}-84 u_{2}^{3} u_{3} u_{6}+210 u_{2}^{2} u_{3}^{2} u_{5}-105 u_{2}^{2} u_{3} u_{4}^{2}+210 u_{2} u_{3}^{3} u_{4}-280 u_{3}^{5}}{u_{2}^{20 / 3}} .
\end{aligned}
$$

Furthermore, in terms of the total differentiation operator:

$$
D_{x}:=\frac{\partial}{\partial x}+u_{1} \frac{\partial}{\partial u}+\sum_{i=1}^{\infty} u_{i+1} \frac{\partial}{\partial u_{i}}
$$

the affine-invariant differentiation operator:

$$
\mathscr{D}_{x}:=\frac{1}{u_{2}^{1 / 3}} D_{x}
$$

enables to produce higher order invariants, for instance:

$$
\mathscr{D}_{x}\left(\frac{1}{3} \frac{-5 u_{3}^{2}+3 u_{2} u_{4}}{u_{2}^{8 / 3}}\right)=\frac{1}{9} \frac{9 u_{2}^{2} u_{5}-45 u_{2} u_{3} u_{4}+40 u_{3}^{3}}{u_{2}^{4}} .
$$

More will be said in Section 15 .

Next, we present an ancient result ([15]).

Lemma 13.10. (1) A curve $u=u(x)$ with $u_{x x} \neq 0$ is affinely equivalent to a parabola $v=y^{2}$ if and only if:

$$
0 \equiv P(u):=\frac{1}{3} \frac{3 u_{x x} u_{x x x x}-5 u_{x x x}^{2}}{u_{x x}^{8 / 3}} .
$$

(2) A curve $u=u(x)$ with $u_{x x} \neq 0$ is affinely equivalent to a nondegenerate conic in the plane if and only if:

$$
0 \equiv C(u):=\frac{1}{9} \frac{9 u_{x x}^{2} u_{x x x x x}-45 u_{x x} u_{x x x} u_{x x x x}+40 u_{x x x}^{3}}{u_{x x}^{4}} .
$$

(3) $P(u) \equiv 0$ implies $C(u) \equiv 0$.

Proof. (1) The target being $v=y^{2}$, we have after an affine transformation whose linear part $\left(\begin{array}{ll}a & b \\ k & l\end{array}\right) \sim\left(\begin{array}{ll}1 & 0 \\ 0 & 1\end{array}\right)$ may be assumed close to the identity:

$$
\mathrm{k} x+\mathrm{l} u+\mathrm{m}=(\mathrm{a} x+\mathrm{b} u+\mathrm{c})^{2},
$$

hence we may solve for $u$ thanks to the positivity of the discriminant:

$$
u=\frac{\mathrm{I}-2 \mathrm{bc}-2 \mathrm{ab} x \pm \sqrt{\left(4 \mathrm{~b}^{2} \mathrm{k}-4 \mathrm{abl}\right) x+\mathrm{l}^{2}-4 \mathrm{bcl}+4 \mathrm{~b}^{2} \mathrm{~m}}}{2 \mathrm{~b}^{2}} .
$$

Thus with different constants the general equation of parabolas is:

$$
u=\mathrm{d} x+\mathrm{e}+\sqrt{2 \mathrm{~g} x+\mathrm{h}} .
$$


At first, in order to eliminate $d$ and e, we just differentiate two times:

$$
u_{x x}=-\frac{\mathrm{g}^{2}}{(2 \mathrm{~g} x+\mathrm{h})^{3 / 2}},
$$

and next, to eliminate the remaining constants, we upside-down:

$$
\frac{1}{u_{x x}^{2 / 3}}=\frac{2}{\mathrm{~g}^{1 / 3}} x+\frac{\mathrm{h}}{\mathrm{g}^{4 / 3}},
$$

and we again differentiate twice:

$$
\left(\frac{1}{u_{x x}^{2 / 3}}\right)_{x x}=-\frac{2}{9} \frac{3 u_{x x} u_{x x x x}-5 u_{x x x}^{2}}{u_{x x}^{8 / 3}} \equiv 0 .
$$

Conversely, from $P(u) \equiv 0$, using $u_{x x} \neq 0$, one reconstitutes by integration (exercise) the general equation $u=\mathrm{d} x+\mathrm{e}+\sqrt{2 \mathrm{~g} x+\mathrm{h}}$.

(2) Now, the target is a general conic in the $\mathbb{R}_{y, v}^{2}$-plane, hence an $x^{2}$ monomial must be present under the square root:

$$
u=\mathrm{d} x+\mathrm{e}+\sqrt{\mathrm{f} x^{2}+2 \mathrm{~g} x+\mathrm{h}} .
$$

Quite similarly:

$$
u_{x x}=\frac{\mathrm{fh}-\mathrm{g}^{2}}{\left(\mathrm{f} x^{2}+2 \mathrm{~g} x+\mathrm{h}\right)^{3 / 2}}
$$

whence:

$$
\frac{1}{u_{x x}^{2 / 3}}=\frac{\mathrm{f}}{\left(\mathrm{f} \mathrm{h}-\mathrm{g}^{2}\right)^{2 / 3}} x^{2}+\frac{2 \mathrm{~g}}{\left(\mathrm{f} \mathrm{h}-\mathrm{g}^{2}\right)^{2 / 3}} x+\frac{\mathrm{h}}{\left(\mathrm{f} \mathrm{h}-\mathrm{g}^{2}\right)^{2 / 3}},
$$

and lastly we have to differentiate three times to get rid of all remaining constants:

$$
\left(\frac{1}{u_{x x}^{2 / 3}}\right)_{x x x}=-\frac{2}{27} \frac{9 u_{x x}^{2} u_{x x x x x}-45 u_{x x} u_{x x x} u_{x x x x}+40 u_{x x x}^{3}}{u_{x x}^{11 / 3}} \equiv 0 .
$$

The converse is also left as an exercise.

(3) follows from a direct differentiation of $0 \equiv 3 u_{x x}(x) u_{x x x x}(x)-5 u_{x x x}(x)^{2}$, and will now be explained in a more theoretical framework.

\section{Recurrence formulas for Differential Invariants}

14.1. Prolongations of infinitesimal generators. As before, let $G$ be a local Lie group of finite dimension $1 \leqslant r<\infty$ acting on graphs in $\mathbb{R}_{x, u}^{p+q}$. Abbreviate $z=(x, u)$ and $z^{(n)}=\left(x, u^{(n)}\right)$. Choose any basis $e_{1}, \ldots, e_{r}$ for the Lie algebra $\mathfrak{g}:=\operatorname{Lie}(G)$ and introduce the $r$ infinitesimal generators of the $G$-action on the considered space:

$$
\mathbf{v}_{\sigma}:=\left.\frac{d}{d t}\right|_{t=0} \exp \left(t e_{\sigma}\right) \cdot z
$$

We thus get $r$ vector fields forming a Lie algebra, which we will write as:

$$
\mathbf{v}_{\sigma}=\sum_{i=1}^{p} \xi_{\sigma}^{i}(x, u) \frac{\partial}{\partial x^{i}}+\sum_{\alpha=1}^{q} \varphi_{\sigma}^{\alpha} \frac{\partial}{\partial u^{\alpha}}
$$


For any jet order $\mathscr{O} \geqslant 0$, and even up to infinity, the prolongations of these fields:

$$
\mathbf{v}_{\sigma}^{(\infty)}=\mathbf{v}_{\sigma}+\sum_{\# J \geqslant 1} \sum_{\alpha=1}^{q} \varphi_{\sigma J}^{\alpha}\left(x, u^{(J)}\right) \frac{\partial}{\partial u_{J}^{\alpha}},
$$

have coefficients computed by applying Theorem 3.7 .

$$
\varphi_{\sigma J}^{\alpha}=\mathrm{D}_{x^{J}}\left(\varphi_{\sigma}^{\alpha}-\sum_{1 \leqslant i \leqslant p} \xi_{\sigma}^{i} u_{i}^{\alpha}\right)+\sum_{1 \leqslant i \leqslant p} \xi_{\sigma}^{i} u_{J, i}^{\alpha},
$$

where, by a slight abuse of notation, we have denoted $u^{(J)}$ instead of $u^{(\# J)}$.

Next, we recall that the modified total differentiation operators are:

$$
\left(\begin{array}{c}
\mathrm{E}_{x^{1}} \\
\vdots \\
\mathrm{E}_{x^{p}}
\end{array}\right):=\left(\begin{array}{ccc}
\mathrm{D}_{x^{1}}\left(y^{1}\right) & \cdots & \mathrm{D}_{x^{1}}\left(y^{p}\right) \\
\vdots & \ddots & \vdots \\
\mathrm{D}_{x^{p}}\left(y^{1}\right) & \cdots & \mathrm{D}_{x^{p}}\left(y^{p}\right)
\end{array}\right)^{-1}\left(\begin{array}{c}
\mathrm{D}_{x^{1}} \\
\vdots \\
\mathrm{D}_{x^{p}}
\end{array}\right)
$$

and after perfoming abstractly the matrix inversion, this means that there exist coefficientfunctions such that:

$$
\mathrm{E}_{x^{i}}=\sum_{1 \leqslant j \leqslant p} Z_{i}^{j}\left(g, x, u^{(1)}\right) \mathrm{D}_{x^{j}} .
$$

Following [13], we may invariantize these $\mathrm{E}_{x^{i}}$ by replacing the group parameters $g=$ $\left(g_{1}, \ldots, g_{r}\right)$ by their values $g:=\rho\left(z^{\left(n_{\mathrm{G}}\right)}\right)$ solved from the cross-section equations, and in this way, we produce $p$ invariant horizontal differential operators:

$$
\left.\mathscr{D}_{i}:=\operatorname{inv}\left(\mathrm{E}_{x^{i}}\right):=\sum_{1 \leqslant j \leqslant p} Z_{i}^{j}\left(\rho\left(z^{\left(n_{\mathrm{G}}\right)}\right), x, u^{(1)}\right)\right) \mathrm{D}_{x^{j}} .
$$

Observation 14.3. Such invariant differential operators $\mathscr{D}_{1}, \ldots, \mathscr{D}_{p}$ have coefficients which depend on jets of order $n_{\mathrm{G}}$, where $n_{\mathrm{G}}$ is the minimal jet order for which the action of $G$ on $J_{x, u}^{n}$ becomes locally foliated (free) of rank equal to $r=\operatorname{dim} G$.

Knowing that a moving frame map $\rho: J_{p, q}^{n_{\mathrm{G}}} \longrightarrow G$ is often difficult to construct explicitly, these $\mathscr{D}_{i}$ are almost never explicit!

Definition 14.4. The invariantization of an arbitrary function $\mathscr{F}=\mathscr{F}\left(z^{(n)}\right)$ defined on any $n^{\text {th }}$ order jet space $J_{x, u}^{n}$ with $n \geqslant 0$ is defined by replacing firstly $z^{(n)}$ in its argument by the target value $w^{(n)}=w^{(n)}\left(g, z^{(n)}\right)$ and secondly the group parameters $g=\left(g_{1}, \ldots, g_{r}\right)$ by their expressions solved from the (not canonical) cross-section equations:

$$
\boldsymbol{F}:=\operatorname{inv}\left(\mathscr{F}\left(z^{(n)}\right)\right):=\mathscr{F}\left(w^{(n)}\left(\rho\left(z^{\left(n_{\mathrm{G}}\right)}\right), z^{(n)}\right)\right) .
$$

Of course, $\operatorname{inv}(\mathscr{F})$ is always a differential invariant. In particular, one can invariantize all jet monomials:

$$
\begin{aligned}
J^{i} & :=\operatorname{inv}\left(x^{i}\right)=y^{i}\left(\rho\left(z^{\left(n_{\mathrm{G}}\right)}\right), x, u\right), \\
I_{K}^{\alpha} & :=\operatorname{inv}\left(u_{K}^{\alpha}\right)=v_{K}^{\alpha}\left(\rho\left(z^{\left(n_{\mathrm{G}}\right)}\right), x, u^{(K)}\right),
\end{aligned}
$$

where, as before, $1 \leqslant i \leqslant p, 1 \leqslant \alpha \leqslant q, K=k_{1}, \ldots, k_{\lambda}, 1 \leqslant k_{1}, \ldots, k_{\lambda} \leqslant p$. We will call them monomial differential invariants.

Convention 14.5. When $K=\emptyset$, we agree that $I_{\emptyset}^{\alpha}=I^{\alpha}$ is the invariantization of the un-differentiated $u^{\alpha}$. 
Most of the times — at least for all group actions considered in this article - , the action of $G$ is transitive on $\mathbb{R}_{x, u}^{p+q}$, hence the cross-section equations can be assumed to contain the $p+q$ equations:

$$
y^{i}=0, \quad u^{\alpha}=0
$$

and then:

$$
\boldsymbol{J}^{i}=0, \quad \boldsymbol{I}^{\alpha}=0 .
$$

We will abbreviate:

$$
I^{(n)}:=\left(J^{i},\left\{I_{K}^{\alpha}\right\}_{0 \leqslant \# K \leqslant n}^{1 \leqslant \alpha \leqslant q}\right)
$$

and we can at last present the fundamental recurrence formulas.

Theorem 14.6. [13] [Recurrence relations] The invariant derivatives of all the monomial invariants $I_{K}^{\alpha}$ with respect to $\mathscr{D}_{1}, \ldots, \mathscr{D}_{p}$ express as differential invariants of order $\# K+1$ raised by one unit:

$$
\mathscr{D}_{j} \boldsymbol{I}_{K}^{\alpha}=\boldsymbol{I}_{K, j}^{\alpha}+\sum_{1 \leqslant \sigma \leqslant r} \varphi_{\sigma K}^{\alpha}\left(\boldsymbol{I}^{(K)}\right) \cdot \boldsymbol{K}_{j}^{\sigma}\left(\boldsymbol{I}^{\left(n_{\mathrm{G}}+1\right)}\right)
$$

with the peculiar case of:

$$
\mathscr{D}_{j} \boldsymbol{J}^{i}=\delta_{j}^{i}+\sum_{1 \leqslant \sigma \leqslant r} \xi_{\sigma}^{i}\left(\boldsymbol{I}^{(0)}\right) \cdot \boldsymbol{K}_{j}^{\sigma}\left(\boldsymbol{I}^{\left(n_{\mathrm{G}}+1\right)}\right)
$$

plus some correction remainders which incorporate the invariantizations $\varphi_{\sigma K}^{\alpha}\left(I^{(K)}\right)$ of the coefficients $\varphi_{\sigma K}^{\alpha}\left(z^{(K)}\right)$ of the prolonged vector fields $\mathbf{v}_{1}^{(\infty)}, \ldots, \mathbf{v}_{r}^{(\infty)}$, as well as certain special differential invariants $K_{j}^{\sigma}$ of order $\leqslant n_{\mathrm{G}}+1$ - called of 'Maurer-Cartan' type.

Moreover, for each fixed $1 \leqslant j \leqslant p$, the linear system of $r$ equations in the $r$ unknows $K_{j}^{1}, \ldots, K_{j}^{r}$ constituted of the $r$ recurrence relations written only for the $r$ phantom invariants:

$$
\operatorname{inv}\left(w_{\nu_{1}}^{\left(n_{1}\right)}\right)=c_{1}, \ldots \ldots, \operatorname{inv}\left(w_{\nu_{r}}^{\left(n_{r}\right)}\right)=c_{r}
$$

has left-hand sides all equal to $0=\mathscr{D}_{j}\left(c_{1}\right)=\cdots=\mathscr{D}_{j}\left(c_{r}\right)$, is of Cramér type, and can be solved uniquely for $K_{j}^{1}, \ldots, K_{j}^{r}$ in terms of the other present differential invariants, all of order at most:

$$
1+\max _{1 \leqslant h \leqslant r} n_{h}=1+n_{\mathrm{G}}
$$

The last sentence justifies why these Maurer-Cartan invariants can be written $K_{j}^{\sigma}=$ $K_{j}^{\sigma}\left(\boldsymbol{I}^{\left(n_{\mathrm{G}}+1\right)}\right)$. They appear in a wider theoretical context $([13])$, but in all applications, the most efficient way to determine these $K_{j}^{\sigma}$ is to set up and solve the mentioned $p$ Cramér systems.

This fundamental theorem has (at least) three outstanding deep qualities.

- To provide all possible differential relations between differential invariants ([13]).

- To be applicable without the need of computing explicitly any — even a single one which is not phantom! - differential invariant, a task which reveals itself desperately beyond the reach of any modern symbolic software. 
- To jump at a synthetic level at which calculations amount to plain linear algebra in low dimension, hence are very accessible.

14.7. Groups containing translations. Now, assume that our local Lie group $G$ contains all translations:

$$
\left(x^{i}, u^{\alpha}\right) \longmapsto\left(x^{i}+\mathrm{a}^{i}, u^{\alpha}+\mathrm{b}^{\alpha}\right) .
$$

This assumption is justified throughout the article, because we will mainly deal with the standard affine or special affine groups of transformations acting on $\mathbb{R}^{p+q}$.

We take a reference point $p_{0} \in \mathbb{R}^{p+q}$, at which we center the coordinates $(x, u)$, so that $p_{0}=(0,0)$ is the origin. With $z=(x, u)$ and $w=(y, v)$, we recall that we denote the $G$-action from $\mathbb{R}^{m}$ to $\mathbb{R}^{m}$ as $z \longmapsto w(g, z)$, where $m=p+q$.

So the initial graphs $\left\{u^{\alpha}=F^{\alpha}(x)\right\}$ we are considering satisfy $F^{\alpha}(0)=0$, while the target $G$-transformed graphs $\left\{v^{\beta}=G^{\beta}(g, y)\right\}$ do not necessarily pass through the origin - but our goal is to reduce ourselves to having $G^{\beta}(g, 0)=0$ too, at the price of reducing $G$. Like for the $G$-structures of the Cartan method of equivalence, this principle of progressive reduction will be practically very powerful.

To this aim, we may introduce the isotropy subgroup of the origin:

$$
G_{0}:=\{g \in G: w(g, 0)=0\} .
$$

It is a local Lie group too, just because fixing a point is preserved by composition. Since our group $G$ which contains all translations is trivially transitive, we have:

$$
\operatorname{dim} G_{0}=r-(p+q)=: r_{0} .
$$

After a reordering, we may assume that a basis for $\mathfrak{g}=\operatorname{Lie}(G)$ as introduced before is organized so that:

$$
\begin{aligned}
\mathfrak{g} & =\operatorname{Span}\left\{e_{1}, \ldots, e_{m}, e_{m+1}, \ldots, e_{r}\right\}, \\
\operatorname{Lie}\left(G_{0}\right)=: \mathfrak{g}_{0} & =\operatorname{Span}\left\{\begin{array}{r}
\left.e_{m+1}, \ldots, e_{r}\right\} .
\end{array}\right.
\end{aligned}
$$

If as before, we also introduce the infinitesimal generators of the action of $G$ on $\mathbb{R}^{m}$ :

$$
\mathbf{v}_{\sigma}:=\left.\frac{d}{d t}\right|_{t=0} \exp \left(t e_{\sigma}\right) \cdot z
$$

$(1 \leqslant \sigma \leqslant r)$

then as is well known since Lie $([10,22])$, all the ones associated to $G_{0}$ do vanish at the origin:

$$
0=\left.\mathbf{v}_{m+1}\right|_{0}=\cdots=\left.\mathbf{v}_{r}\right|_{0} .
$$

Since $G$ contains all translations, it is clear that we may assume that the first $p+q$ infinitesimal generators are plainly:

$$
\mathbf{v}_{i}=\partial_{x^{i}}, \quad \mathbf{v}_{p+\alpha}=\partial_{u^{\alpha}} \quad(1 \leqslant i \leqslant p, 1 \leqslant \alpha \leqslant q) .
$$

Furthermore, on the jet space $J_{x, u}^{n_{\mathrm{G}}}$ of the appropriate order $n_{\mathrm{G}}$, we may assume that the cross-section equations to the lifted action of $G$ on $J_{x, u}^{n_{\mathrm{G}}}$ contain the $(p+q)$ equations:

$$
y^{i}(g, x, u)=0, \quad v^{\alpha}(g, x, u)=0,
$$

and that these equations are the first $m$ among the $r$ cross-section equations we wrote abstractly as:

$$
0=w_{\nu_{1}=1}^{\left(n_{1}\right)}(z, g)=\cdots=w_{\nu_{m}=m}^{\left(n_{m}\right)}(z, g) .
$$


Therefore, among the phantom differential invariants, there are the $p+q$ :

$$
\boldsymbol{J}^{i}=\operatorname{inv}\left(y^{i}\right), \quad \boldsymbol{I}_{\emptyset}^{\alpha}=\operatorname{inv}\left(v^{\alpha}\right) .
$$

A quick inspection of the prolongation formulas of Theorem 3.7 convinces of an

Observation 14.8. The prolongations of the translational vector fields are all trivial:

$$
\mathbf{v}_{i}^{(\infty)}=\mathbf{v}_{i}+0, \quad \mathbf{v}_{p+\alpha}^{(\infty)}=\mathbf{v}_{p+\alpha}+0 .
$$

Then in the fundamental recurrence formulas of Theorem 14.6, we decide to set aside the $p+q$ ones concerning these first phantom invariants, namely:

$$
\begin{aligned}
& \mathscr{D}_{j} \boldsymbol{J}^{i}=\delta_{j}^{i}+1 \cdot \boldsymbol{K}_{j}^{i}+\sum_{m+1 \leqslant \sigma \leqslant r} \xi_{\sigma}^{i}\left(\boldsymbol{I}^{(0)}\right) \cdot \boldsymbol{K}_{j}^{\sigma}\left(\boldsymbol{I}^{\left(n_{\mathrm{G}}+1\right)}\right), \\
& \mathscr{D}_{j} \boldsymbol{I}_{\emptyset}^{\alpha}=\boldsymbol{I}_{j}^{\alpha}+1 \cdot \boldsymbol{K}_{j}^{p+\alpha}+\sum_{m+1 \leqslant \sigma \leqslant r} \varphi_{\sigma}^{\alpha}\left(\boldsymbol{I}^{(0)}\right) \cdot \boldsymbol{K}_{j}^{\sigma}\left(\boldsymbol{I}^{\left(n_{\mathrm{G}}+1\right)}\right),
\end{aligned}
$$

and to conserve only the recurrence relations that are of order $\# K \geqslant 1$ :

$$
\begin{aligned}
\mathscr{D}_{j} I_{K}^{\alpha} & =I_{K, j}^{\alpha}+\sum_{1 \leqslant \sigma \leqslant r} \varphi_{\sigma K}^{\alpha}\left(I^{(K)}\right) \cdot K_{j}^{\sigma}\left(I^{\left(n_{\mathrm{G}}+1\right)}\right) \\
& =I_{K, j}^{\alpha}+0+\sum_{m+1 \leqslant \sigma \leqslant r} \varphi_{\sigma K}^{\alpha}\left(I^{(K)}\right) \cdot K_{j}^{\sigma}\left(I^{\left(n_{\mathrm{G}}+1\right)}\right)
\end{aligned}
$$

but then our observation that $\varphi_{\sigma K}^{\alpha}=0$ or all $1 \leqslant \sigma \leqslant m$ shows that $m$ terms drop in all the correction sums.

If we now write only the $r-m$ recurrence formulas which concern the remaining $r-m$ phantom differential invariants:

$$
\operatorname{inv}\left(w_{\nu_{m+1}}^{\left(n_{m+1}\right)}(z, g)\right), \ldots \ldots, \operatorname{inv}\left(w_{\nu_{r}}^{\left(n_{r}\right)}(z, g)\right)
$$

we receive for every fixed $j$ a system of $r-m$ linear equations in the $r-m$ MaurerCartan invariants $K_{j}^{m+1}, \ldots, K_{j}^{r}$. This system is a subsystem of the full $r \times r$ system of Theorem 14.6 for $K_{j}^{1}, \ldots, K_{j}^{m}, K_{j}^{m+1}, \ldots, K_{j}^{r}$, and by triangularity (by blocks), the $(r-m) \times(r-m)$ subsystem is also of Cramér type, hence can be solved uniquely for $\boldsymbol{K}_{j}^{m+1}, \ldots, \boldsymbol{K}_{j}^{r}$ in terms of $\boldsymbol{I}^{\left(n_{\mathrm{G}}+1\right)}$.

14.9. Groups containing translations and transvections. Assume now that $G$ contains not only translations, but also all vertical transvections:

$$
v^{\alpha}=u^{\alpha}+\mathrm{q}_{1}^{\alpha} x^{1}+\cdots+\mathrm{q}_{p}^{\alpha} x^{p}
$$

Thus, $\operatorname{dim} G=r \geqslant p+q+p q$. The $p q$ infinitesimal generators are:

$$
x^{i} \partial_{u^{\alpha}}
$$

$(1 \leqslant i \leqslant p, 1 \leqslant \alpha \leqslant q)$.

An application of Theorem 3.7 shows that their infinite prolongations are truncated after first order:

$$
\left(x^{i} \partial_{u^{\alpha}}\right)^{(\infty)}=x^{i} \partial_{u^{\alpha}}+\partial_{u_{i}^{\alpha}}+0 .
$$

We list the first $p+q+p q$ infinitesimal generators as:

$$
\mathbf{v}_{i}:=\partial_{x^{i}}, \quad \quad \mathbf{v}_{p+\alpha}:=\partial_{u^{\alpha}}, \quad\left\{\mathbf{v}_{p+q+1}, \ldots, \mathbf{v}_{p+q+p q}\right\}=\left\{x^{i} \partial_{u^{\alpha}}\right\} .
$$


Hence we have:

$$
\begin{aligned}
(1 \leqslant \sigma \leqslant p+q, \quad \# K \geqslant 1) & \Longrightarrow \quad \varphi_{\sigma K}^{\alpha}=0 \\
(p+q+1 \leqslant \sigma \leqslant p+q+p q, \quad \# K \geqslant 2) & \Longrightarrow \quad \varphi_{\sigma K}^{\alpha}=0 .
\end{aligned}
$$

Among the cross-section equations, we may take:

$$
y^{i}=0, \quad v^{\alpha}=0, \quad v_{i}^{\alpha}=0 .
$$

This conducts to $p+q+p q$ phantom invariants:

$$
J^{i}=0, \quad I_{\emptyset}^{\alpha}=0, \quad I_{i}^{\alpha}=0 .
$$

Then in the fundamental recurrence formulas of Theorem 14.6, we decide to set aside the $p+q+p q$ ones concerning these first phantom invariants, and the interesting recurrence relations are, for any $\# K \geqslant 2$ :

$$
\begin{aligned}
\mathscr{D}_{j} I_{K}^{\alpha} & =I_{K, j}^{\alpha}+\sum_{1 \leqslant \sigma \leqslant r} \varphi_{\sigma K}^{\alpha}\left(I^{(K)}\right) \cdot K_{j}^{\sigma}\left(\boldsymbol{I}^{\left(n_{\mathrm{G}}+1\right)}\right) \\
& =I_{K, j}^{\alpha}+0+\sum_{m_{1}+1 \leqslant \sigma \leqslant r} \varphi_{\sigma K}^{\alpha}\left(\boldsymbol{I}^{(K)}\right) \cdot \boldsymbol{K}_{j}^{\sigma}\left(\boldsymbol{I}^{\left(n_{\mathrm{G}}+1\right)}\right),
\end{aligned}
$$

where we abbreviate $m_{1}:=p+q+p q$.

It remains $r-m_{1}$ infinitesimal generators $\mathbf{v}_{m_{1}+1}, \ldots, \mathbf{v}_{r}$. If we now write only the $r-m_{1}$ recurrence formulas which concern the remaining $r-m_{1}$ phantom differential invariants:

$$
\operatorname{inv}\left(w_{\nu_{m_{1}+1}}^{\left(n_{m_{1}+1}\right)}(z, g)\right), \ldots \ldots, \operatorname{inv}\left(w_{\nu_{r}}^{\left(n_{r}\right)}(z, g)\right)
$$

we receive for every fixed $j$ a system of $r-m_{1}$ linear equations in the $r-m_{1}$ MaurerCartan invariants $K_{j}^{m_{1}+1}, \ldots, K_{j}^{r}$. This system is a subsystem of the full $r \times r$ system of Theorem 14.6 for $K_{j}^{1}, \ldots, K_{j}^{m_{1}}, K_{j}^{m_{1}+1}, \ldots, K_{j}^{r}$, and by triangularity (by blocks), the $\left(r-m_{1}\right) \times\left(r-m_{1}\right)$ subsystem is also of Cramér type, hence can be solved uniquely for $\boldsymbol{K}_{j}^{m_{1}+1}, \ldots, \boldsymbol{K}_{j}^{r}$ in terms of $\boldsymbol{I}^{\left(n_{\mathrm{G}}+1\right)}$. This observation will be useful later on, in Section 20.

\section{Moving Frame Method and Recurrence Relations for Curves}

In general, the structure of the full algebra of differential invariants can be understood thanks to the recurrence formulas obtained by Fels and Olver in [13].

For any integer $n \geqslant 0$, let $J_{x, u}^{n}$ be the space of $n^{\text {th }}$ order jets of functions $\mathbb{R} \ni x \longmapsto$ $u(x) \in \mathbb{R}$, equipped with coordinates:

$$
\left(x, u, u_{1}, \ldots, u_{n}\right),
$$

where the $u_{i}$ stand for $\frac{\partial^{i} u}{\partial x^{i}}(x)$, as abstract independent variables.

According to [22, Ch. 23] and to [30, 24], the $n^{\text {th }}$ prolongation of a general vector field on the $(x, u)$ space:

is a vector field on $J_{x, u}^{n}$ :

$$
\mathbf{v}=\xi(x, u) \frac{\partial}{\partial x}+\eta(x, u) \frac{\partial}{\partial u}
$$

$$
\mathbf{v}^{(n)}:=\mathbf{v}+\sum_{1 \leqslant i \leqslant n} \Phi^{i}\left(x, u, u_{1}, \ldots, u_{i}\right) \frac{\partial}{\partial u_{i}},
$$


which has extended coefficients uniquely defined as:

$$
\Phi^{i}:=\underbrace{\mathrm{D}_{x}\left(\cdots \left(\mathrm{D}_{x}\right.\right.}_{i \text { times }}\left(\eta-\xi u_{1}\right)) \cdots)+\xi u_{i+1}
$$

In [24], one finds closed explicit formulas for all these coefficients $\Phi^{i}$ in any jet order $n \geqslant 1$, even for an arbitrary number of independent variables $\left(x^{1}, \ldots, x^{p}\right)$ and for an arbitrary number of dependent variables $\left(u^{1}, \ldots, u^{q}\right)$. However, in small dimensions and for small jet order, it is better and almost straightforward to capture these $\Phi^{i}$ using a computer.

In the present context, the group $\mathrm{SL}_{2}(\mathbb{R})$ has 3 natural infinitesimal generators:

$$
\mathbf{v}_{1}:=x \partial_{x}-u \partial_{u}, \quad \mathbf{v}_{2}:=u \partial_{x}, \quad \mathbf{v}_{3}:=x \partial_{u},
$$

whose prolongations to the $n^{\text {th }}$ order jet space are, as a computer tells us:

$$
\begin{aligned}
\mathbf{v}_{1}^{(n)}=x \partial_{x} & -u \partial_{u}-2 u_{1} \partial_{u_{1}}-3 u_{2} \partial_{u_{2}}-4 u_{3} \partial_{u_{3}}-\cdots-(n+1) u_{n} \partial_{u_{n}}, \\
\mathbf{v}_{2}^{(n)}=u \partial_{x} & -u_{1}^{2} \partial_{u_{1}}-3 u_{1} u_{2} \partial_{u_{2}}-\left(4 u_{1} u_{3}+3 u_{2}^{2}\right) \partial_{u_{3}}-\left(5 u_{1} u_{4}+10 u_{2} u_{3}\right) \partial_{u_{4}}- \\
& -\left(6 u_{1} u_{5}+15 u_{2} u_{4}+10 u_{3}^{2}\right) \partial_{u_{5}}-\left(7 u_{1} u_{6}+21 u_{2} u_{5}+35 u_{3} u_{4}\right) \partial_{u_{6}}- \\
& -\left(8 u_{1} u_{7}+28 u_{2} u_{6}+56 u_{3} u_{5}+35 u_{4}^{2}\right) \partial_{u_{7}}-\cdots, \\
\mathbf{v}_{3}^{(n)}= & x \partial_{u}+\partial_{u_{1}},
\end{aligned}
$$

For any jet order $n \geqslant 1$, we would write:

$$
\mathbf{v}_{\kappa}^{(n)}=\mathbf{v}_{\kappa}+\sum_{1 \leqslant i \leqslant n} \Phi_{\kappa}^{i} \partial_{u_{i}}
$$

and reading the first terms of $\mathbf{v}_{1}^{(n)}, \mathbf{v}_{3}^{(n)}$, it is visible that:

$$
\Phi_{3}^{(1)}=1, \quad \Phi_{3}^{(2)}=\cdots=\Phi_{3}^{(n)}=0, \quad \Phi_{1}^{(k)}=-(k+1) u_{k} \quad(1 \leqslant k \leqslant n),
$$

while it can be proved (exercise) that when $n=: 2 n$ is even:

$$
\Phi_{2}^{2 n}=-\sum_{1 \leqslant k \leqslant n}\left(\begin{array}{c}
2 n+1 \\
k
\end{array}\right) u_{k} u_{2 n+1-k}
$$

and when $n=: 2 n+1$ is odd:

$$
\Phi_{2}^{2 n+1}=-\sum_{1 \leqslant k \leqslant n}\left(\begin{array}{c}
2 n+2 \\
k
\end{array}\right) u_{k} u_{2 n+2-k}-\frac{1}{2}\left(\begin{array}{c}
2 n+2 \\
n+1
\end{array}\right)\left(u_{n+1}\right)^{2} .
$$

Fels and Olver in [13] introduced an invariantization operator 'inv' which transforms every function on the jet space into a differential invariant. After having centered our surface at the origin by means of a translation, and after having set to constant three other Taylor coefficients as in Theorem 13.8, we come to:

$$
\begin{array}{rlrl}
\operatorname{inv}(x) & =0, & & \operatorname{inv}(u)=0, \\
I_{1}:=\operatorname{inv}\left(u_{x}\right)=0, & I_{2}:=\operatorname{inv}\left(u_{x x}\right)=1, & I_{3}:=\operatorname{inv}\left(u_{x x x}\right)=0 .
\end{array}
$$

Generally, every pure jet monomial produces a differential invariant:

$$
I_{k}:=\operatorname{inv}\left(u_{x^{k}}\right)
$$

$(k \geqslant 0)$,

whose expression is most of the time lengthy and complex, while the 5 constantified invariants $\operatorname{inv}(x), \operatorname{inv}(u), I_{1}, I_{2}, I_{3}$ are called phantom invariants. 
The recurrence formulas obtained by Fels and Olver in [13] can be set up without knowing the (huge) explicit expressions of differential invariants and are of the form:

$$
I_{k+1}=\mathscr{D}_{x}\left(I_{k}\right)-\sum_{1 \leqslant \kappa \leqslant 3} \operatorname{inv}\left(\Phi_{\kappa}^{k}\right) R^{\kappa} \quad(k \geqslant 0),
$$

where the invariant differentiation operator $\mathscr{D}_{x}$ already introduced above in $(13.9)$ is a nonzero multiple of the total differentiation operator $\mathrm{D}_{x}$, where all invariantized coefficients of the prolonged vector fields are simply:

$$
\operatorname{inv}\left(\Phi_{\kappa}^{k}\left(x, u, u_{1}, u_{2}, u_{3}, u_{4}, \ldots, u_{k}\right)\right)=\Phi_{\kappa}^{k}\left(0,0,0,1,0, I_{4}, \ldots, I_{k}\right)
$$

due to the fundamental commutation:

$$
\text { invariantization } \circ \text { function }=\text { function } \circ \text { invariantization, }
$$

and where the Maurer-Cartan coefficients $R^{1}, R^{2}, R^{3}$ - which are also differential invariants — can be determined by solving the linear system composed of the three recurrence relations applied to the three phantom invariants, technically as follows.

One writes the $3 \times 3$ matrix of the coefficients of the three generators $\mathbf{v}_{1}, \mathbf{v}_{2}, \mathbf{v}_{3}$ of $\mathfrak{s l}_{2}(\mathbb{R})$ with respect to the three basic fields $\partial_{u_{1}}, \partial_{u_{2}}, \partial_{u_{3}}$ corresponding to the three invariants $\operatorname{inv}\left(u_{1}\right), \operatorname{inv}\left(u_{2}\right), \operatorname{inv}\left(u_{3}\right)$ which were phantom:

$$
\begin{array}{ccc}
\partial_{u_{1}} & \partial_{u_{2}} & \partial_{u_{3}} \\
\mathbf{v}_{1} \\
\mathbf{v}_{2}\left(\begin{array}{ccc}
-2 u_{1} & -3 u_{2} & -4 u_{3} \\
-u_{1}^{2} & -3 u_{1} u_{2} & -4 u_{1} u_{3}-3 u_{2}^{2} \\
\mathbf{v}_{3} & 0 & 0
\end{array} \quad \stackrel{\text { invariantize }}{\longrightarrow}\right.
\end{array} \quad \text { ( }
$$

one invariantizes this matrix, one transposes it, and one gets the phantom recurrence relations (15.1) for $k=1,2,3$ :

$$
\left(\begin{array}{l}
\boldsymbol{I}_{2} \\
\boldsymbol{I}_{3} \\
\boldsymbol{I}_{4}
\end{array}\right)=\left(\begin{array}{l}
\mathscr{D}_{x}\left(\boldsymbol{I}_{1}\right) \\
\mathscr{D}_{x}\left(\boldsymbol{I}_{2}\right) \\
\mathscr{D}_{x}\left(\boldsymbol{I}_{3}\right)
\end{array}\right)-\left(\begin{array}{ccc}
0 & 0 & 1 \\
-3 & 0 & 0 \\
0 & -3 & 0
\end{array}\right)\left(\begin{array}{l}
R^{1} \\
R^{2} \\
R^{3}
\end{array}\right),
$$

which, if we abbreviate the parabolas invariant as:

$$
P:=\operatorname{inv}\left(u_{x x x x}\right)
$$

become:

$$
\left(\begin{array}{l}
1 \\
0 \\
P
\end{array}\right)=\left(\begin{array}{l}
0 \\
0 \\
0
\end{array}\right)-\left(\begin{array}{ccc}
0 & 0 & 1 \\
-3 & 0 & 0 \\
0 & -3 & 0
\end{array}\right)\left(\begin{array}{l}
R^{1} \\
R^{2} \\
R^{3}
\end{array}\right)
$$

and this simple linear system has as the unique solution:

$$
R^{1}:=0 \quad R^{2}:=\frac{1}{3} P, \quad R^{3}:=-1 .
$$

Once these three Maurer-Cartan invariants have been determined, they can be plugged in all the other recurrence formulas (15.1). In particular for $k=4,5$, determining first the 
coefficients of $\partial_{u_{4}}, \partial_{u_{5}}$ :

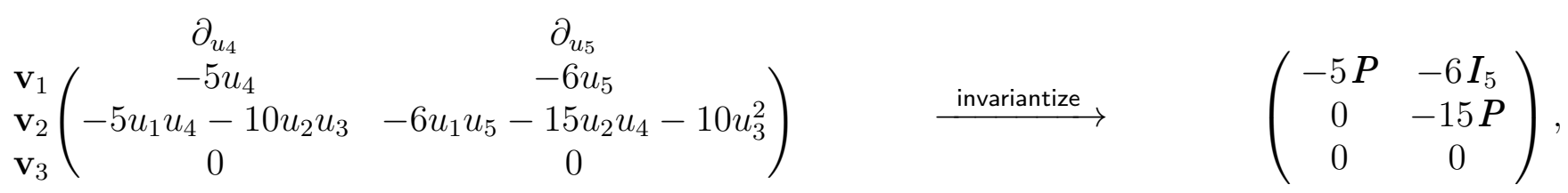

we reach an invariant which we abbreviate as:

$$
\begin{aligned}
M:=I_{5} & =\mathscr{D}_{x}(P)-(-5 P) \cdot 0-0 \cdot \frac{1}{3} P-0 \cdot(-1) \\
& =\mathscr{D}_{x}(P), \\
I_{6} & =\mathscr{D}_{x}\left(I_{5}\right)-(-6 M) \cdot 0-(-15 P) \cdot \frac{1}{3} P-0 \cdot(-1) \\
& =\mathscr{D}_{x}\left(\mathscr{D}_{x}(P)\right)+5 P^{2} .
\end{aligned}
$$

These first two formulas suggest that the parabolas invariant $P$ together with all its invariants derivatives $\mathscr{D}_{x}^{\nu} P$ generate the algebra of differential invariants, a result established by Olver in [36]. Let us explain this a bit more.

Indeed, if we abbreviate the relevant invariantizations of the coefficients of $\mathbf{v}_{2}^{(n)}$ as:

$$
\Lambda_{k}:=\operatorname{inv}\left(\Phi_{2}^{k}\right)
$$

$(k \geqslant 4)$,

then we have:

$$
\begin{array}{lll}
\Lambda_{4}=0, & I_{5}=\mathscr{D}_{x} I_{4}+\frac{P}{3} \Lambda_{4}=\mathscr{D}_{x} P, \\
\Lambda_{5}=15 I_{4}, & I_{6}=\mathscr{D}_{x} I_{5}+\frac{P}{3} \Lambda_{5}=\mathscr{D}_{x}^{2} P+5 P^{2}, \\
\Lambda_{6}=21 I_{5}, & I_{7}=\mathscr{D}_{x} I_{6}+\frac{P}{3} \Lambda_{6}=\mathscr{D}_{x}^{3} P+17 \mathscr{D}_{x} P P, \\
\Lambda_{7}=28 I_{6}+35 I_{4}^{2}, & I_{8}=\mathscr{D}_{x}^{4} P+17\left(\mathscr{D}_{x} P\right)^{2}+\frac{79}{3} \mathscr{D}_{x}^{2} P P+\frac{175}{3} P^{3}, \\
\Lambda_{8}=36 I_{7}+126 I_{4} I_{5}, & I_{9}=\mathscr{D}_{x}^{5} P+\frac{181}{3} \mathscr{D}_{x}^{2} P \mathscr{D}_{x} P+\frac{115}{3} \mathscr{D}_{x}^{3} P P+421 \mathscr{D}_{x} P P^{2},
\end{array}
$$

Although it is clear that $\left\{\mathscr{D}_{x}^{\nu} P\right\}_{\nu \in \mathbb{N}}$ generates the full algebra of differential invariants of curves under $\mathrm{SA}_{2}(\mathbb{R})$, it is an open question ([36]) to get closed explicit formulas for all the $I_{k}$.

In summary, what we have done in our basic Section $\mathbf{1 3}$ is to decompose into successive steps — stratified by increasing jet orders viewed in power series centered at the origin all the elimination computations which are required to normalize plane curves under special affine transformations, while the famous moving frame method proceeds with a choice of a suitable cross-section in a sufficiently high order jet space so as to perform all the elimination computations in one stroke.

Of course, the two processes are mathematically equivalent, but the interest to decompose computations into steps is to be able to grasp some explicit expressions of at least a few differential invariants, and in dimension 2 for parabolic surfaces $S^{2} \subset \mathbb{R}^{3}$, we will soon see that an explicit knowledge of certain 'bifurcating' invariants is necessary in order to study the full ramified tree of equivalent classes.

\section{Affine Differential Invariants of Curves in $\mathbb{R}^{2}$}

But before we pass to dimension 2, let us show how the story changes much when considering the full affine group $A_{2}(\mathbb{R})$, with $6>5$ parameters. In fact, such a study is not 
artificial, for it will be required to complete our understanding of a certain (thin) branch in our later study of parabolic surfaces $S^{2} \subset \mathbb{R}^{2}$ modulo the special affine group $\mathrm{SA}_{3}(\mathbb{R})$.

As all symbolic computation softwares do handle polynomials very efficiently, we will employ the power series method, and we will proceed as explained in the beginning of Section 13 .

16.1. First loop. Leaving aside the condition $a d-b c=1$, we therefore start with general $\mathrm{GL}_{2}(\mathbb{R})$ matrices:

$$
\left(\begin{array}{ll}
a & b \\
c & d
\end{array}\right)
$$$$
(\mathrm{ad}-\mathrm{bc} \neq 0) \text {. }
$$

With $x=\mathrm{a} y+\mathrm{b} v$ and $u=\mathrm{c} y+\mathrm{d} v$, the fundamental equation writes again:

$$
0 \equiv-\mathrm{c} y-\mathrm{d} G(y)+F(\mathrm{a} y+\mathrm{b} G(y)) \text {. }
$$

As for $\mathrm{SL}_{2}(\mathbb{R})$, we may assume that first-order terms are normalized to vanish:

$$
u=\mathrm{O}_{x}(2) \quad \text { and } \quad v=\mathrm{O}_{y}(2) .
$$

Lemma 16.3. The subgroup of $\mathrm{GL}_{2}(\mathbb{R})$ sending $v=\mathrm{O}_{y}(2)$ to $u=\mathrm{O}_{x}(2)$ is 3-dimensional and consists of matrices:

$$
G_{\text {stab }}^{(1)}: \quad\left(\begin{array}{ll}
a & b \\
0 & d
\end{array}\right) \quad(a d \neq 0) .
$$

Next, assuming of course still that $F_{2} \neq 0 \neq G_{2}$ in:

$$
u=F_{2} \frac{x^{2}}{2 !}+\mathrm{O}_{x}(3) \quad \text { and } \quad v=G_{2} \frac{y^{2}}{2 !}+\mathrm{O}_{y}(3),
$$

we examine how this reduced group $G_{\text {stab }}^{(1)}$ acts on the second order term $y^{2}$ of the fundamental equation 16.2 :

$$
0 \equiv\left(-\mathrm{d} G_{2}+\mathrm{a}^{2} F_{2}\right) \frac{y^{2}}{2 !}+\mathrm{O}_{y}(3) .
$$

Consequently, we can make $G_{2}:=1$ for the curve in the target space $\mathbb{R}_{y, v}^{2}$ by choosing $\mathrm{d}:=\mathrm{a}^{2} F_{2}$, and with the simplest choice $\mathrm{a}:=1$, we apply the invertible matrix:

$$
\left(\begin{array}{cc}
1 & 0 \\
0 & F_{2}
\end{array}\right) \in G_{\text {stab }}^{(1)}
$$

Calculations of further terms in the fundamental equation (16.2) provide:

$$
G_{3}=\frac{F_{3}}{F_{2}}, \quad G_{4}=\frac{F_{4}}{F_{2}}, \quad G_{5}=\frac{F_{5}}{F_{2}},
$$

and if we come back to initial functional jets, we get new values:

$$
G_{2}:=1, \quad G_{3}:=\frac{u_{3}}{u_{2}}, \quad G_{4}:=\frac{u_{4}}{u_{2}}, \quad G_{5}:=\frac{u_{5}}{u_{2}} .
$$

16.4. Second loop. We restart from equations normalized up to order 2 :

$$
u=\frac{x^{2}}{2 !}+F_{3} \frac{x^{3}}{3 !}+\cdots, \quad v=\frac{y^{2}}{2 !}+G_{3} \frac{x^{3}}{3 !}+\cdots .
$$

To determine the subgroup $\mathrm{G}_{\text {stab }}^{(2)} \subset \mathrm{G}_{\text {stab }}^{(1)}$ which stabilizes these normalizations, we compute the first term in the fundamental equation (16.2):

$$
0 \equiv\left(-\mathrm{d}+\mathrm{a}^{2}\right) \frac{y^{2}}{2 !}+\mathrm{O}_{y}(3) \text {. }
$$


Lemma 16.5. The subgroup of $\mathrm{GL}_{2}(\mathbb{R})$ sending $v=\frac{y^{2}}{2 !}+\mathrm{O}_{y}(3)$ to $u=\frac{x^{2}}{2 !}+\mathrm{O}_{x}(3)$ is 2-dimensional and consists of matrices:

$$
G_{\text {stab }}^{(2)}: \quad\left(\begin{array}{cc}
a & b \\
0 & a^{2}
\end{array}\right) \quad(a \neq 0) .
$$

Next, we examine how this reduced group $\mathrm{G}_{\mathrm{stab}}^{(2)}$ acts on the third order term $y^{3}$ :

$$
0 \equiv\left(-\mathrm{a}^{2} G_{3}+3 \mathrm{ab}+\mathrm{a}^{3} F_{3}\right) \frac{y^{3}}{3 !}+\mathrm{O}_{y}(4) .
$$

Consequently, we can make $G_{3}:=0$ by choosing $\mathrm{b}:=-\frac{1}{3} \mathrm{a}^{2} F_{3}$, and with the simple matrix:

$$
\left(\begin{array}{cc}
1 & -\frac{1}{3} F_{3} \\
0 & 1
\end{array}\right)
$$

we transform $\{u=F(x)\}$ into:

$$
v=\frac{y^{2}}{2 !}+0+\left(F_{4}-\frac{5}{3} F_{3}^{2}\right) \frac{y^{4}}{4 !}+\left(F_{5}-5 F_{3} F_{4}+\frac{40}{9} F_{3}^{3}\right) \frac{y^{5}}{5 !}+\mathrm{O}_{y}(6) .
$$

Lastly, coming back to the initial functional jets, we get new values:

$$
\begin{aligned}
G_{2}:=1, \quad G_{3}:=0, \quad G_{4} & :=F_{4}-\frac{5}{3} F_{3}^{2}=\frac{1}{3} \frac{-5 u_{3}^{2}+3 u_{2} u_{4}}{u_{2}^{2}}, \\
G_{5} & :=F_{5}-5 F_{3} F_{4}+\frac{40}{9} F_{3}^{3}=\frac{1}{9} \frac{9 u_{2}^{2} u_{5}-45 u_{2} u_{3} u_{4}+40 u_{3}^{3}}{u_{2}^{3}} .
\end{aligned}
$$

16.6. Third loop. We restart from equations normalized up to order 3 :

$$
u=\frac{x^{2}}{2 !}+0+F_{4} \frac{x^{4}}{4 !}+\cdots, \quad v=\frac{y^{2}}{2 !}+0+G_{4} \frac{y^{4}}{4 !}+\cdots .
$$

To determine the subgroup $\mathrm{G}_{\text {stab }}^{(3)} \subset \mathrm{G}_{\text {stab }}^{(2)}$ which stabilizes these normalizations, we compute the first term in the fundamental equation 16.2 :

$$
0 \equiv(3 \mathrm{ab}) \frac{y^{3}}{3 !}+\mathrm{O}_{y}(4)
$$

Lemma 16.7. The subgroup of $\mathrm{GL}_{2}(\mathbb{R})$ sending $v=\frac{y^{2}}{2 !}+0+\mathrm{O}_{y}(4)$ to $u=\frac{x^{2}}{2 !}+0+\mathrm{O}_{x}(4)$ is 1-dimensional and consists of matrices:

$$
G_{\text {stab }}^{(3)} \quad\left(\begin{array}{cc}
a & 0 \\
0 & a^{2}
\end{array}\right) \quad(a \neq 0) .
$$

Next, we examine how this reduced group $\mathrm{G}_{\text {stab }}^{(3)}$ acts on the third order term $y^{4}$ :

$$
0 \equiv\left(-\mathrm{a}^{2} G_{4}+\mathrm{a}^{4} F_{4}\right) \frac{y^{4}}{4 !}+\mathrm{O}_{y}(5) \text {. }
$$

At this stage, because a $\neq 0$, a bifurcation necessarily occurs, according to whether $F_{4}=0$ or $F_{4} \neq 0$. In fact, coming back to functional jets:

$$
F_{4}=\frac{1}{3} \frac{-5 u_{3}^{2}+3 u_{2} u_{4}}{u_{2}^{2}}
$$

the branch $F_{4} \equiv 0$ must be interpreted as an identical vanishing in the $4^{\text {th }}$ order jet space, and we have the elementary 
Lemma 16.8. A plane curve $\{u=F(x)\}$ with $F_{x x} \neq 0$ is affinely equivalent to the model parabola $\left\{v=y^{2}\right\}$ if and only if:

$$
0 \equiv \frac{1}{3} \frac{-5 F_{x x x}^{2}+3 F_{x x} F_{x x x x}}{F_{x x}^{2}} .
$$

Proof. A possible proof has already been left as an exercise in Lemma 13.10, but it is enlightening to provide another proof which requires no integration, only basic differential algebra, $c f$. [26, Thm. 4.1].

The ordinary differential equation $0 \equiv-5 F_{x x x}^{2}+3 F_{x x} F_{x x x x}$ is invariant not only under the $\mathrm{SA}_{2}(\mathbb{R})$ action, but also under general affine transformations, for one can prove directly ([26, p. 26]) that if a graph $\{u=F(x)\}$ is transformed into a graph $\{v=G(y)\}$ through:

$$
x=\mathrm{a} y+\mathrm{b} v+\mathrm{c}, \quad u=\mathrm{k} y+\mathrm{l} v+\mathrm{m} \quad(\mathrm{al}-\mathrm{b} \neq 0),
$$

then:

$$
\begin{aligned}
F_{x x} & =\frac{\mathrm{al}-\mathrm{bk}}{\left(\mathrm{a}+\mathrm{b} G_{y}\right)^{3}} G_{y y}, \\
-5 F_{x x x}^{2}+3 F_{x x} F_{x x x x} & =\frac{(\mathrm{al}-\mathrm{bk})^{2}}{\left(\mathrm{a}+\mathrm{b} G_{y}\right)^{8}}\left[-5 G_{y y y}^{2}+3 G_{y y} G_{y y y y}\right] .
\end{aligned}
$$

Therefore, after some preliminary affine transformation as done in Section $\mathbf{1 3}$, we can assume that the equation of our curve is already normalized as:

$$
v=G(y)=y^{2}+0+\mathrm{O}_{y}(4) .
$$

Again, we have $0 \equiv-5 G_{y y y}^{2}+3 G_{y y} G_{y y y y}$ and $G_{y y} \neq 0$ as well. To conclude, it would then suffice to show that the remainder $\mathrm{O}_{y}(4) \equiv 0$ vanishes identically - very easy!

Indeed, we can solve:

$$
G_{y y y y}=\frac{5}{3} \frac{G_{y y y}^{2}}{G_{y y}},
$$

and from $G_{y y y}(0)=0$, we deduce $G_{y y y y}(0)=0$. Let us abbreviate this relation as:

$$
G_{y y y y}=\mathscr{R} G_{y y y}
$$

where $\mathscr{R}=\mathscr{R}(y)$ denotes an unspecified function. Then we may differentiate it and replace:

$$
G_{\text {yyyyy }}=\mathscr{R}_{y} G_{\text {yyy }}+\mathscr{R} G_{\text {yyy }}=\mathscr{R} G_{y y y}
$$

to deduce similarly $G_{\text {yyyyy }}(0)=0$. Obviously, induction yields $G_{y^{k}}=\mathscr{R} G_{y y y}$ for any $k \geqslant 4$, whence $G_{y^{k}}(0)=0$, and we conclude $\mathrm{O}_{y}(4) \equiv 0$, since we assume analyticity.

The case $F_{4}=0$ being now fully understood, we can assume that $F_{4} \neq 0$, and then, two symmetric subcases occur:

$$
F_{4}>0 \quad \text { and } \quad F_{4}<0 .
$$

Assuming $F_{4}>0$, we can then make $G_{4}:=1$ by means of the simple matrix:

$$
\left(\begin{array}{cc}
F_{4}^{-1 / 2} & 0 \\
0 & F_{4}^{-1}
\end{array}\right) .
$$

Looking at next terms in the fundamental equation, we get:

$$
G_{5}:=\frac{F_{5}}{F_{4}^{3 / 2}}, \quad G_{6}:=\frac{F_{6}}{F_{4}^{2}}, \quad G_{7}:=\frac{F_{7}}{F_{4}^{5 / 2}} \text {. }
$$


Coming back to functional jets:

$$
\begin{aligned}
& F_{5}:=\frac{1}{\sqrt{3}} \frac{9 u_{2}^{2} u_{5}-45 u_{2} u_{3} u_{4}+40 u_{3}^{3}}{\left(3 u_{2} u_{4}-5 u_{3}^{2}\right)^{3 / 2}} \\
& F_{6}:=\frac{9 u_{2}^{3} u_{6}-63 u_{2}^{2} u_{3} u_{5}+105 u_{2} u_{3}^{2} u_{4}-35 u_{3}^{4}}{\left(3 u_{2} u_{4}-5 u_{3}^{2}\right)^{2}} .
\end{aligned}
$$

16.9. Fourth loop. The subgroup stabilizing equations normalized up to order 4 :

$$
u=\frac{x^{2}}{2 !}+0+\frac{x^{4}}{4 !}+\cdots, \quad v=\frac{y^{2}}{2 !}+0+\frac{y^{4}}{4 !}+\cdots .
$$

is easily seen to reduce to the identity. The algorithm therefore stops, and a first result, valid only at the level of power series at the origin, is a corollary of this reduction to an $\{e\}$-group.

Theorem 16.10. (1) Given a real analytic curve $\{u=F(x)\}$ in $\mathbb{R}^{2}$ passing through the origin which satisfies:

$$
F_{x x}(0) \neq 0 \quad \text { and } \quad \pm\left(F_{x x} F_{x x x x}-\frac{5}{3} F_{x x x}^{2}\right)>0,
$$

there always exist a $\mathrm{GL}_{2}(\mathbb{R})$ transformation which puts it into the form

$$
u=\frac{x^{2}}{2 !}+0 \pm \frac{x^{4}}{4 !}+F_{5} \frac{x^{5}}{5 !}+\sum_{i \geqslant 6} F_{i} \frac{x^{i}}{i !} .
$$

(2) Any other such real analytic curve $\{v=G(y)\}$ similarly put into the form:

$$
v=\frac{y^{2}}{2 !}+0 \pm \frac{y^{4}}{4 !}+G_{5} \frac{y^{5}}{5 !}+\sum_{j \geqslant 6} G_{j} \frac{y^{j}}{j !} .
$$

is $\mathrm{GL}_{2}(\mathbb{R})$-equivalent to $\{u=F(x)\}$ above if and only if all Taylor coefficients match:

$$
G_{5}=F_{5}, \quad G_{i}=F_{i} \quad(\forall i \geqslant 6) .
$$

16.11. Recurrence relations. Similarly as in Section 15, let us set up the corresponding recurrence relations. By Theorem 16.10, we come to:

$$
\begin{aligned}
& \operatorname{inv}(x)=0, \\
& \operatorname{inv}(u)=0, \\
& I_{1}:=\operatorname{inv}\left(u_{x}\right)=0, \\
& I_{2}:=\operatorname{inv}\left(u_{x x}\right)=1, \\
& I_{3}:=\operatorname{inv}\left(u_{x x x}\right)=0, \\
& I_{4}:=\operatorname{inv}\left(u_{x x x x}\right)= \pm 1 .
\end{aligned}
$$

We take the 4 infinitesimal generators of the the action of $\mathrm{GL}_{2}(\mathbb{R}) \subset \mathrm{A}_{2}(\mathbb{R})$ on $\mathbb{R}_{x, u}^{2}$ :

$$
\mathbf{v}_{1}:=x \partial_{x}, \quad \mathbf{v}_{2}:=u \partial_{u}, \quad \mathbf{v}_{3}:=u \partial_{x}, \quad \mathbf{v}_{4}:=x \partial_{u}
$$

the prolongations of which we write as:

$$
v_{\kappa}^{(\infty)}=v_{\kappa}+\sum_{k \geqslant 0} \Phi_{\kappa}^{k}\left(x, u, u_{1}, \ldots, u_{k}\right) \frac{\partial}{\partial u_{k}}
$$

Generally, every pure jet monomial produces a differential invariant:

$$
I_{k}:=\operatorname{inv}\left(u_{x^{k}}\right)
$$

$(k \geqslant 0)$,

and the recurrence formulas are of the form:

$$
I_{k+1}=\mathscr{D}_{x}\left(I_{k}\right)-\sum_{1 \leqslant \kappa \leqslant 4} \operatorname{inv}\left(\Phi_{\kappa}^{k}\right) R^{\kappa} \quad(k \geqslant 0),
$$


for some uniquely defined invariant differentiation operator $\mathscr{D}_{x}$ which is a nonzero multiple of the total differentiation operator $\mathrm{D}_{x}$, and where, as before, all invariantized coefficients of the prolonged vector fields are simply:

$$
\operatorname{inv}\left(\Phi_{\kappa}^{k}\left(x, u, u_{1}, u_{2}, u_{3}, u_{4}, u_{5}, \ldots, u_{k}\right)\right)=\Phi_{\kappa}^{k}\left(0,0,0,1,0, \pm 1, I_{5}, \ldots, I_{k}\right),
$$

and where the Maurer-Cartan invariants $R^{1}, R^{2}, R^{3}, R^{4}$ can be determined as follows.

One writes the $4 \times 4$ matrix of the coefficients of the four generators $\mathbf{v}_{1}, \mathbf{v}_{2}, \mathbf{v}_{3}, \mathbf{v}_{4}$ of $\mathfrak{g l}_{2}(\mathbb{R})$ with respect to the four basic fields $\partial_{u_{1}}, \partial_{u_{2}}, \partial_{u_{3}}, \partial_{u_{4}}$ corresponding to the four invariants $\operatorname{inv}\left(u_{1}\right), \operatorname{inv}\left(u_{2}\right), \operatorname{inv}\left(u_{3}\right), \operatorname{inv}\left(u_{4}\right)$ which were phantom:

\begin{tabular}{|c|c|c|c|c|c|c|c|}
\hline$\partial_{u_{1}}$ & $\partial_{u_{2}}$ & $\partial_{u_{3}}$ & $\partial_{u_{4}}$ & & & & \\
\hline$J_{1} /-u_{1}$ & $-2 u_{2}$ & $-3 u_{3}$ & $-4 u_{4}$ & & $\begin{array}{ll}0 & -2\end{array}$ & 0 & 干 \\
\hline $\begin{array}{ll}\mathbf{v}_{2} & u_{1}\end{array}$ & $u_{2}$ & $u_{3}$ & $u_{4}$ & invariantize & 1 & 0 & \pm 1 \\
\hline${ }_{3}-u_{1}^{2}$ & $-3 u_{1} u_{2}$ & $-4 u_{1} u_{3}-3 u_{2}^{2}$ & $-5 u_{1} u_{4}-10 u_{2} u_{3}$ & & 0 & -3 & 0 \\
\hline 1 & 0 & 0 & 0 & & 1 & 0 & 0 \\
\hline
\end{tabular}

one invariantizes this matrix, one transposes it, and one gets the phantom recurrence relations for $k=1,2,3,4$ :

$$
\left(\begin{array}{l}
\boldsymbol{I}_{2} \\
\boldsymbol{I}_{3} \\
\boldsymbol{I}_{4} \\
\boldsymbol{I}_{5}
\end{array}\right)=\left(\begin{array}{l}
\mathscr{D}_{x}\left(\boldsymbol{I}_{1}\right) \\
\mathscr{D}_{x}\left(\boldsymbol{I}_{2}\right) \\
\mathscr{D}_{x}\left(\boldsymbol{I}_{3}\right) \\
\mathscr{D}_{x}\left(\boldsymbol{I}_{4}\right)
\end{array}\right)-\left(\begin{array}{cccc}
0 & 0 & 0 & 1 \\
-2 & 1 & 0 & 0 \\
0 & 0 & -3 & 0 \\
\mp 4 & \pm 1 & 0 & 0
\end{array}\right)\left(\begin{array}{l}
R^{1} \\
R^{2} \\
R^{3} \\
R^{4}
\end{array}\right)
$$

which become:

$$
\left(\begin{array}{c}
1 \\
0 \\
\pm 1 \\
I_{5}
\end{array}\right)=\left(\begin{array}{l}
0 \\
0 \\
0 \\
0
\end{array}\right)-\left(\begin{array}{cccc}
0 & 0 & 0 & 1 \\
-2 & 1 & 0 & 0 \\
0 & 0 & -3 & 0 \\
\mp 4 & \pm 1 & 0 & 0
\end{array}\right)\left(\begin{array}{l}
R^{1} \\
R^{2} \\
R^{3} \\
R^{4}
\end{array}\right)
$$

and this simple linear system has as the unique solution:

$$
R^{1}:= \pm \frac{1}{2} I_{5}, \quad R^{2}:= \pm I_{5}, \quad R^{3}:= \pm \frac{1}{3}, \quad R^{4}:=-1 .
$$

Once these four Maurer-Cartan invariants have been determined, they can be plugged in:

$$
\begin{gathered}
\partial_{u_{5}} \\
\mathbf{v}_{1} \\
\mathbf{v}_{2} \\
\mathbf{v}_{3} \\
\mathbf{v}_{4}
\end{gathered}\left(\begin{array}{cc}
\partial_{u_{6}} \\
u_{5} & -6 u_{6} \\
-6 u_{1} u_{5}-15 u_{2} u_{4}-10 u_{3}^{2} & -7 u_{1} u_{6}-21 u_{2} u_{5}-35 u_{3} u_{4} \\
0 & 0
\end{array}\right) \stackrel{\text { invariantize }}{\longrightarrow}\left(\begin{array}{cc}
-5 I_{5} & -6 \boldsymbol{I}_{6} \\
\boldsymbol{I}_{5} & \boldsymbol{I}_{6} \\
\mp 15 & -21 \boldsymbol{I}_{5} \\
0 & 0
\end{array}\right) .
$$

We deduce:

$$
\begin{aligned}
& I_{6}=\mathscr{D}_{x}\left(\boldsymbol{I}_{5}\right) \pm \frac{3}{2} \boldsymbol{I}_{5}^{2}+5, \\
& \boldsymbol{I}_{7}=\mathscr{D}_{x}\left(\boldsymbol{I}_{6}\right) \pm 2 \boldsymbol{I}_{5} \boldsymbol{I}_{6} \pm 7 \boldsymbol{I}_{5},
\end{aligned}
$$

and it is easy to verify that the algebra of differential invariants is generated by $I_{5}$ and its invariant derivatives $\mathscr{D}_{x}^{\nu}\left(\boldsymbol{I}_{5}\right)$ of any order $\nu \geqslant 1$. 


\section{Parabolic Surfaces $S^{2} \subset \mathbb{R}^{3}$ : Invariant $W$ of Order 4}

Recall from Section $\mathbf{1 0}$ that we are interested in parabolic surfaces $S^{2} \subset \mathbb{R}^{3}$ graphed as $\{u=F(x, y)\}$, whose Hessian matrix is degenerate, of rank $1<2$, whence after a rotation in the $(x, y)$-space, we may assume:

$$
F_{x x} \neq 0 \equiv F_{x x} F_{y y}-F_{x y}^{2}
$$

In the same Section 10 , we also showed that the relation $F_{y y}=\frac{F_{x y}^{2}}{F_{x x}}$ has infinitely many differential consequences, namely, for every $(j, k)$ with $k \geqslant 2$, there exists a certain universal rational expressions $R_{j, k}$ such that:

$$
F_{x^{j} y^{k}}=R_{j, k}\left(\left\{F_{j^{\prime}, 0}\right\}_{0 \leqslant j^{\prime} \leqslant j+k},\left\{F_{j^{\prime \prime}, 1}\right\}_{0 \leqslant j^{\prime \prime} \leqslant j+k-1}\right)
$$

the denominators of these $R_{j, k}$ being certain powers $\left(F_{x x}\right)^{*}$. Inside the order $n$ jet space $J_{x, u}^{n}$, this means that:

$$
u_{j, k}=R_{j, k}\left(\left\{u_{j^{\prime}, 0}\right\}_{0 \leqslant j^{\prime} \leqslant j+k},\left\{u_{j^{\prime \prime}, 1}\right\}_{0 \leqslant j^{\prime \prime} \leqslant j+k-1}\right) .
$$

Theorem 8.5 already showed that the assumption that the Hessian determinant is identically zero is stable under (special) affine transformations. It is then interesting to have a confirmation of this basic fact by means of prolongations of vector fields.

We start by selecting an appropriate basis for the 11-dimensional Lie algebra of infinitesimal generators of the action of $\mathrm{SA}_{3}(\mathbb{R})$ on the space $\mathbb{R}_{x, y, u}^{3}$, and we choose the same generators as in [33]:

$$
\begin{gathered}
\mathbf{v}_{\mathbf{1}}:=x \partial_{x}-u \partial_{u}, \quad \mathbf{v}_{2}:=y \partial_{y}-u \partial_{u}, \\
\mathbf{v}_{3}:=y \partial_{x}, \quad \mathbf{v}_{4}:=u \partial_{x}, \quad \mathbf{v}_{5}:=x \partial_{y}, \quad \mathbf{v}_{6}:=u \partial_{y}, \quad \mathbf{v}_{7}:=x \partial_{u}, \quad \mathbf{v}_{8}:=y \partial_{u}, \\
\mathbf{w}_{1}:=\partial_{x}, \quad \mathbf{w}_{2}:=\partial_{y}, \quad \mathbf{w}_{3}:=\partial_{u} .
\end{gathered}
$$

Among these 11 vector fields, 5 are essentially useless, namely the three translational $\mathbf{w}_{1}, \mathbf{w}_{2}, \mathbf{w}_{3}$, and the two transvectional $\mathbf{v}_{7}, \mathbf{v}_{8}$, as was already explained and understood by Theorem 12.6. A confirmation of this fact is provided by a computation of the prolongations of these 5 vector fields to any order:

$$
\begin{aligned}
& \partial_{x} \quad \partial_{y} \quad \partial_{u} \quad \partial_{u_{1,0}} \quad \partial_{u_{0,1}} \quad\left\{\partial_{u_{k, l}}\right\}_{2 \leqslant k+l} \\
& \begin{array}{l}
\mathbf{v}_{7} \\
\mathbf{v}_{8} \\
\mathbf{w}_{1} \\
\mathbf{w}_{2} \\
\mathbf{w}_{3}
\end{array}\left(\begin{array}{llllll}
0 & 0 & x & 1 & 0 & 0 \\
0 & 0 & y & 0 & 1 & 0 \\
1 & 0 & 0 & 0 & 0 & 0 \\
0 & 1 & 0 & 0 & 0 & 0 \\
0 & 0 & 1 & 0 & 0 & 0
\end{array}\right),
\end{aligned}
$$

with absolutely zero coefficients in front of all $\partial_{u_{j, k}}$ with $j+k \geqslant 2$. Up to a permutation of rows and columns, we thus have a matrix which is triangular-by-blocks $5=2+3$, essentially the $5 \times 5$ identity matrix, followed by a zero matrix.

Consequently, if, for any fixed jet order $n \geqslant 2$, above any point $(x, y)$, we want to determine:

$$
\operatorname{rank} \operatorname{Span}\left(\mathbf{v}_{1}^{(n)}, \mathbf{v}_{2}^{(n)}, \mathbf{v}_{3}^{(n)}, \mathbf{v}_{4}^{(n)}, \mathbf{v}_{5}^{(n)}, \mathbf{v}_{6}^{(n)}, \mathbf{v}_{7}^{(n)}, \mathbf{v}_{8}^{(n)}, w_{1}^{(n)}, w_{2}^{(n)}, w_{3}^{(n)}\right),
$$


it suffices to determine the rank of only 6 prolonged vector fields $\mathbf{v}_{1}^{(n)}, \ldots, \mathbf{v}_{6}^{(n)}$, with firstorder parts truncated/dropped:

$$
\begin{aligned}
& \partial_{u_{2,0}} \quad \partial_{u_{1,1}} \quad \partial_{u_{3,0}} \quad \partial_{u_{2,1}} \quad \partial_{u_{1,2}} \quad \partial_{u_{0,3}} \quad\left\{\partial_{u_{k, l}}\right\}_{4 \leqslant k+l} \\
& \begin{array}{lllllll}
\mathbf{v}_{1} \\
\mathbf{v}_{2} \\
\mathbf{v}_{3} \\
\mathbf{v}_{4} \\
\mathbf{v}_{5} \\
\mathbf{v}_{6}
\end{array}
\end{aligned}
$$

Let us denote the submanifold $\left\{u_{j, k}=R_{j, k}\right\}$ of parabolic jets as:

$$
P J_{2,1}^{n} \subset J_{2,1}^{n}
$$

with:

$$
\operatorname{dim} P J_{2,1}^{n}=3+2 n<2+\left(\begin{array}{c}
2+n \\
n
\end{array}\right)=\operatorname{dim} J_{2,1}^{n},
$$

the inequality being strict as soon as $n \geqslant 2, e . g$. when $n=4$ :

$$
11<17
$$

with the strange coincidence that this dimension 11 of $P J_{2,1}^{4}$ is equal to $\operatorname{dim} \mathrm{SA}_{3}(\mathbb{R})$ ! A nice surprise is waiting for us about that!

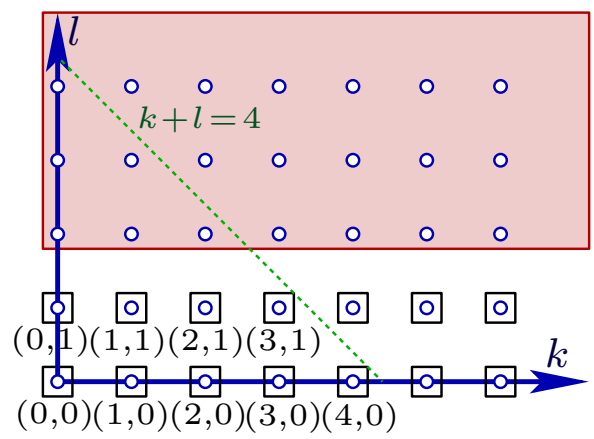

Also, let us introduce the projection from the full jet space onto the parabolic jet space:

$$
\begin{aligned}
\operatorname{pj}\left(x, y,\left\{u_{j, k}\right\}_{j+k \leqslant n}\right) & :=\left(x, y, \begin{array}{ccccc}
u_{0,1}, & \ldots & \ldots & u_{n-1,1}, \\
& u, & u_{1,0}, & \ldots, & u_{n-1,0}, u_{n, 0}
\end{array}\right) \\
& \in \mathbb{R}^{3+2 n} .
\end{aligned}
$$

A general vector field:

$$
\mathbf{v}=\xi(x, y, u) \frac{\partial}{\partial x}+\eta(x, y, u) \frac{\partial}{\partial y}+\varphi(x, y, u) \frac{\partial}{\partial u},
$$

prolongs infinitely as:

$$
\mathbf{v}^{\infty}=\mathbf{v}+\sum_{1 \leqslant j+k} \Phi^{j, k}\left(x, y, u^{(j+k)}\right) \frac{\partial}{\partial u_{j, k}},
$$


with coefficients $\Phi^{j, k}$ given by mean of the two (infinite, commuting) total differentiation operators:

$$
\begin{aligned}
& \mathrm{D}_{x}:=\frac{\partial}{\partial x}+u_{1,0} \frac{\partial}{\partial u}+\sum_{1 \leqslant j+k} u_{j+1, k} \frac{\partial}{\partial u_{j, k}}, \\
& \mathrm{D}_{y}:=\frac{\partial}{\partial y}+u_{0,1} \frac{\partial}{\partial u}+\sum_{1 \leqslant j+k} u_{j, k+1} \frac{\partial}{\partial u_{j, k}},
\end{aligned}
$$

by the formulas of Theorem 3.7 ,

$$
\Phi^{j, k}:=\mathrm{D}_{x}^{j} \mathrm{D}_{y}^{k}\left(\varphi-\xi u_{1,0}-\eta u_{0,1}\right)+\xi u_{j+1, k}+\eta u_{j, k+1},
$$

are functions on $J_{2,1}^{j+k}$.

Assertion 17.3. For any jet order $n \geqslant 2$, all the 11 prolonged vector field on $J_{2,1}^{n}$ :

$$
\mathbf{v}_{1}^{(n)}, \quad \mathbf{v}_{2}^{(n)}, \quad \mathbf{v}_{3}^{(n)}, \quad \mathbf{v}_{4}^{(n)}, \quad \mathbf{v}_{5}^{(n)}, \quad \mathbf{v}_{6}^{(n)}, \quad \mathbf{v}_{7}^{(n)}, \quad \mathbf{v}_{8}^{(n)}, \quad \mathbf{w}_{1}^{(n)}, \quad \mathbf{w}_{2}^{(n)}, \quad \mathbf{w}_{3}^{(n)},
$$

are tangent to the (smooth, graphed) submanifold $P J_{2,1}^{n} \subset J_{2,1}^{n}$ of parabolic jets.

Proof. By differentiating one-parameter subgroups, this is a consequence of Theorem 8.5 .

Next, the push-forward to the (horizontal) space $\mathbb{R}^{3+2 n}$ of parabolic jets of such a prolonged vector field is obtained by just 'killing' all the $\partial / \partial u_{j, k}$ having $k \geqslant 2$ :

$$
\operatorname{pj}_{*}\left(\mathbf{v}^{(\infty)}\right)=\mathbf{v}+\sum_{1 \leqslant j^{\prime}} \operatorname{pj}_{*}\left(\Phi^{j^{\prime}, 0}\right) \frac{\partial}{\partial u_{j^{\prime}, 0}}+\sum_{0 \leqslant j^{\prime \prime}} \operatorname{pj}_{*}\left(\Phi^{j^{\prime \prime}, 1}\right) \frac{\partial}{\partial u_{j^{\prime \prime}, 1}},
$$

and when we restrict ourselves to a finite order $n \geqslant 0$, this becomes:

$$
\operatorname{pj}_{*}\left(\mathbf{v}^{(n)}\right)=\mathbf{v}+\sum_{1 \leqslant j^{\prime} \leqslant n} \operatorname{pj}_{*}\left(\Phi^{j^{\prime}, 0}\right) \frac{\partial}{\partial u_{j^{\prime}, 0}}+\sum_{0 \leqslant j^{\prime \prime} \leqslant n-1} \operatorname{pj}_{*}\left(\Phi^{j^{\prime \prime}, 1}\right) \frac{\partial}{\partial u_{j^{\prime \prime}, 1}},
$$

where $\operatorname{pj}_{*}\left(\Phi^{j^{\prime}}\right)$ and $\operatorname{pj}_{*}\left(\Phi^{j^{\prime \prime}, 1}\right)$ are push-forwards of $\Phi^{j^{\prime}, 0}$ and $\Phi^{j^{\prime \prime}, 1}$ to $P J_{2,1}^{j^{\prime}}$ and $P J_{2,1}^{j^{\prime \prime}+1}$.

To summarize, the push-forward process consists of

- dropping all $\partial / \partial u_{j, k}$ with $k \geqslant 2$;

- in the kept coefficients:

$$
\Phi^{j^{\prime}, 0}=\Phi^{j^{\prime}, 0}\left(x, y, u^{\left(j^{\prime}\right)}\right), \quad \Phi^{j^{\prime \prime}, 1}=\Phi^{j^{\prime \prime}, 1}\left(x, y, u^{\left(j^{\prime \prime}+1\right)}\right),
$$

replacing all jet coordinates $u_{j^{\prime \prime \prime}, k^{\prime \prime \prime}}$ with $k^{\prime \prime \prime} \geqslant 2$ by $R_{j^{\prime \prime \prime}, k^{\prime \prime \prime}}$.

For confirmation, for confidence, for coherence, the following observation may (for fun) be checked on whatever computer for many low jet orders $n \geqslant 2$.

Observation 17.4. [Exercise] In order to push forward the 11 generators of the action of $\mathrm{SA}_{3}(\mathbb{R})$ via $\mathrm{pj}_{*}$, it suffices to know parabolic jets relations only for $k=2$, and for any $j \geqslant 0$ :

$$
u_{j, 2}=R_{j, 2}\left(\left\{u_{j^{\prime}, 0}\right\}_{2 \leqslant j^{\prime} \leqslant j+2}, \quad\left\{u_{j^{\prime \prime}, 1}\right\}_{1 \leqslant j^{\prime \prime} \leqslant j+1}\right) .
$$

Observation 17.5. [Exercise] The process of replacing $u_{j, 2}$ by $R_{j, 2}$ occurs only when one calculates $\mathrm{pj}_{*}\left(\mathbf{v}_{5}^{(n)}\right)$ and $\mathrm{pj}_{*}\left(\mathbf{v}_{6}^{(n)}\right)$ for $n \geqslant 2$. 
Now, although Theorem 8.5 already gave a full explanation, we want to verify in another way that the hypothesis that the Hessian is identically zero is stable under (special) affine transformations. So we fix the jet order $n=2$, whence parabolic jets are defined by a single equation:

$$
P J_{2,1}^{2}=\left\{u_{2,0} u_{0,2}-u_{1,1}^{2}\right\}
$$

and equivalently, because we are working on the space $\left\{u_{2,0} \neq 0\right\}$, we can solve:

$$
u_{0,2}=\frac{u_{1,1}^{2}}{u_{2,0}}
$$

Clearly, $\mathbf{v}_{7}^{(2)}, \mathbf{v}_{8}^{(2)}, \mathbf{w}_{1}^{(2)}, \mathbf{w}_{2}^{(2)}, \mathbf{w}_{3}^{(3)}$ are (trivially) tangent to this submanifold $P J_{2,1}^{2} \subset J_{2,1}^{2}$, since they incorporate only derivatives $\partial_{x}, \partial_{y}, \partial_{u}, \partial_{u_{1,0}}, \partial_{u_{0,1}}$, nothing of higher order $\partial_{u_{j, k}}$ with $j+k \geqslant 2$.

Next, we must verify that the 6 remaining vector fields $\mathbf{v}_{1}^{(2)}, \mathbf{v}_{2}^{(2)}, \mathbf{v}_{3}^{(2)}, \mathbf{v}_{4}^{(2)}, \mathbf{v}_{5}^{(2)}, \mathbf{v}_{6}^{(2)}$ are also tangent to $P J_{2,1}^{n}$. We write their coefficients matrix as:

$$
\left.\begin{array}{c} 
\\
\mathbf{v}_{1} \\
\mathbf{v}_{2} \\
\mathbf{v}_{3} \\
\mathbf{v}_{4} \\
\mathbf{v}_{5} \\
\mathbf{v}_{6}
\end{array} \quad \begin{array}{ccc}
\partial_{u_{2,0}} & \partial_{u_{1,1}} & \partial_{u_{0,2}} \\
-3 u_{2,0} & -2 u_{1,1} & -u_{0,2} \\
-u_{2,0} & -2 u_{1,1} & -3 u_{0,2} \\
0 & -u_{2,0} & -2 u_{1,1} \\
-3 u_{2,0} u_{1,0} & -2 u_{1,1} u_{1,0}-u_{2,0} u_{0,1} & -2 u_{1,1} u_{0,1}-u_{0,2} u_{1,0} \\
-2 u_{1,1} & -u_{0,2} & 0
\end{array}\right) .
$$

Also, let us abbreviate:

$$
H_{u}:=u_{2,0} u_{0,2}-u_{1,1}^{2} \text {. }
$$

The tangency in question is verified on a computer, or else with a pen, as follows.

Assertion 17.6. The quotients:

$$
\mathbf{v}_{\sigma}\left(H_{u}\right) / H_{u}
$$

have values, for $\sigma=1,2,3,4,5,6$ :

$$
-4, \quad-4, \quad 0, \quad-4 u_{1,0}, \quad 0, \quad-4 u_{0,1} \text {. }
$$

Thus, $H_{u}=0$ implies $\mathbf{v}_{\sigma}\left(H_{u}\right)=0$, which is tangency! One also observes that there is a $2 \times 2$ minor of the above matrix which is nowhere vanishing:

$$
\left|\begin{array}{cc}
-u_{2,0} & -2 u_{1,1} \\
0 & -u_{2,0}
\end{array}\right|=u_{2,0}^{2}
$$

Question 17.7. Are there differential invariants of order 2?

No! Because at every point of $\left\{u_{2,0} \neq 0=H_{u}\right\}$, we realize that:

$$
\begin{aligned}
& \operatorname{dim} P J_{2,1}^{2}=7=\operatorname{rank} \operatorname{Span}\left(\mathrm{pj}_{*}\left(\mathbf{v}_{2}^{(2)}\right), \operatorname{pj}_{*}\left(\mathbf{v}_{3}^{(2)}\right), \operatorname{pj}_{*}\left(\mathbf{v}_{7}^{(2)}\right), \operatorname{pj}_{*}\left(\mathbf{v}_{8}^{(2)}\right),\right. \\
& \left.\mathrm{pj}_{*}\left(\mathbf{w}_{1}^{(2)}\right), \mathrm{pj}_{*}\left(\mathbf{w}_{2}^{(2)}\right), \mathrm{pj}_{*}\left(\mathbf{w}_{3}^{(2)}\right)\right),
\end{aligned}
$$


by looking at the triangular-by-blocks $7=2+2+3$ matrix:

\begin{tabular}{|c|c|c|c|c|c|c|c|}
\hline & $\partial_{x}$ & $\partial_{y}$ & $\partial_{u}$ & $\partial_{u_{1,0}}$ & $\partial_{u_{0,1}}$ & $\partial_{u_{2,0}}$ & $\partial_{u_{1,1}}$ \\
\hline $\mathbf{v}_{2}$ & 0 & $y$ & $-u$ & $-u_{1,0}$ & $-2 u_{0,1}$ & $-u_{2,0}$ & $\left.-2 u_{1,1}\right)$ \\
\hline $\mathbf{v}_{3}$ & $y$ & 0 & 0 & 0 & $-u_{1,0}$ & 0 & $-u_{2,0}$ \\
\hline $\mathbf{v}_{7}$ & 0 & 0 & $x$ & 1 & 0 & 0 & 0 \\
\hline $\mathbf{v}_{8}$ & 0 & 0 & $y$ & 0 & 1 & 0 & 0 \\
\hline $\mathbf{w}_{1}$ & 1 & 0 & 0 & 0 & 0 & 0 & 0 \\
\hline $\mathbf{w}_{2}$ & 0 & 1 & 0 & 0 & 0 & 0 & 0 \\
\hline $\mathbf{w}_{3}$ & 0 & 0 & 1 & 0 & 0 & 0 & 0 \\
\hline
\end{tabular}

whose determinant equals $u_{2,0}^{2} \neq 0$. Consequently, the set we are working on:

$$
\left\{u_{2,0} \neq 0=u_{2,0} u_{0,2}-u_{1,1}^{2}\right\}
$$

contains only 7-dimension orbits, in fact only a unique orbit, for the prolonged action of $\mathrm{SA}_{3}(\mathbb{R})$. So any transversal to the orbits is zero-dimensional, and there are no differential invariants of order 2. In particular, the Hessian $H_{u}$ is not a differential invariant. Taking the notations of Section 4 , we introduce a

Terminology 17.8. For the action of a local Lie group $G$ on graphs $\{u=\mathrm{u}(x)\}$ in $\mathbb{R}_{z}^{p+q}$, a function $P=P\left(z^{(n)}\right)$ on the $n^{\text {th }}$ order jet space $J_{z}^{n}$ is called a relative invariant if:

$$
P\left(w^{(n)}\left(g, z^{(n)}\right)\right)=\text { nonzero } \cdot P\left(z^{(n)}\right) \quad(\forall g \in G) .
$$

Immediately, the zero-set of a relative invariant is invariant:

$$
\left\{\boldsymbol{P}\left(w^{(n)}\left(g, z^{(n)}\right)\right)=0\right\}=\left\{P\left(z^{(n)}\right)=0\right\} .
$$

Precisely what we already observed of the Hessian!

Next, we examine what occurs in the $3^{\text {rd }}$ order jet space. Recall that we are working only in the domain:

$$
\left\{u_{2,0} \neq 0=u_{2,0} u_{0,2}-u_{1,1}^{2}\right\} \text {. }
$$

The submanifold $P J_{2,1}^{3} \subset J_{2,1}^{3}$ of parabolic $3^{\text {rd }}$ order jets is now defined by three equations (the last one being in fact not used):

$$
\begin{aligned}
u_{0,2}:=\frac{u_{1,1}^{2}}{u_{2,0}} \quad u_{1,2} & =2 \frac{u_{1,1} u_{2,1}}{u_{2,0}}-\frac{u_{1,1}^{2} u_{3,0}}{u_{2,0}^{2}}, \\
u_{0,3} & =3 \frac{u_{1,1}^{2} u_{2,1}}{u_{2,0}^{2}}-2 \frac{u_{1,1}^{3} u_{3,0}}{u_{2,0}^{3}} .
\end{aligned}
$$

Beyond Theorem 8.5, again with affine transformations not far from the identity, we have the

Proposition 17.9. [26, 5.8] With this hypothesis, in terms of the nowhere vanishing quantity:

it holds:

$$
\Upsilon:=\left(\mathrm{I}+\mathrm{m} F_{y}\right) F_{x x}-\left(\mathrm{k}+\mathrm{m} F_{x}\right) F_{x y}
$$

$$
\frac{G_{s s} G_{s s t}-G_{s t} G_{s s s}}{G_{s s}^{2}}=\frac{F_{x x}}{\Upsilon}\left(\frac{F_{x x} F_{x x y}-F_{x y} F_{x x x}}{F_{x x}^{2}}\right) .
$$


This implies that, in the submanifold $P J_{2,1}^{3}$ of parabolic jets of order 3 , the zero-set:

$$
\left\{u_{2,0} u_{2,1}-u_{1,1} u_{3,0}=0\right\},
$$

is invariant under the prolongation of the $\mathrm{SA}_{3}$-action, namely, for every $g \in \mathrm{SA}_{3}(\mathbb{R})$ not far from the identity:

$$
g^{(3)}\left(\left\{u_{2,0} u_{2,1}-u_{1,1} u_{3,0}=0\right\}\right) \subset\left\{v_{2,0} v_{2,1}-v_{1,1} v_{3,0}=0\right\} .
$$

We now want to see the same property from the vector fields point of view. Let us abbreviate:

$$
\boldsymbol{S}_{u}:=\frac{u_{2,0} u_{2,1}-u_{1,1} u_{3,0}}{u_{2,0}^{2}}
$$

The tangencies to $\left\{S_{u}=0\right\}$ follow from an

Assertion 17.11. The quotients:

$$
\operatorname{pj}_{*}\left(\mathbf{v}_{\sigma}\right)\left(S_{u}\right) / S_{u}
$$

have values, for $\sigma=1,2,3,4,5,6$ :

$$
0, \quad-1, \quad 0, \quad 0, \quad \frac{u_{1,1}}{u_{2,0}}, \quad \frac{u_{1,1} u_{1,0}-u_{2,0} u_{0,1}}{u_{2,0}} .
$$

Question 17.12. Are there differential invariants of order 3 ?

Again: No! Because at every point of $\left\{u_{2,0} \neq 0=H_{u}\right\}$, we realize that:

$$
\begin{aligned}
& \operatorname{dim} P J_{2,1}^{3}=9=\operatorname{rank} \operatorname{Span}\left(\mathrm{pj}_{*}\left(\mathbf{v}_{1}^{(3)}\right), \mathrm{pj}_{*}\left(\mathbf{v}_{2}^{(3)}\right), \operatorname{pj}_{*}\left(\mathbf{v}_{3}^{(3)}\right), \mathrm{pj}_{*}\left(\mathbf{v}_{4}^{(3)}\right),\right. \\
& \left.\mathrm{pj}_{*}\left(\mathbf{v}_{7}^{(3)}\right), \mathrm{pj}_{*}\left(\mathbf{v}_{8}^{(3)}\right), \mathrm{pj}_{*}\left(\mathbf{w}_{1}^{(3)}\right), \mathrm{pj}_{*}\left(\mathbf{w}_{2}^{(3)}\right), \mathrm{pj}_{*}\left(\mathbf{w}_{3}^{(3)}\right)\right),
\end{aligned}
$$

by looking at the triangular-by-blocks $9=4+2+3$ matrix:

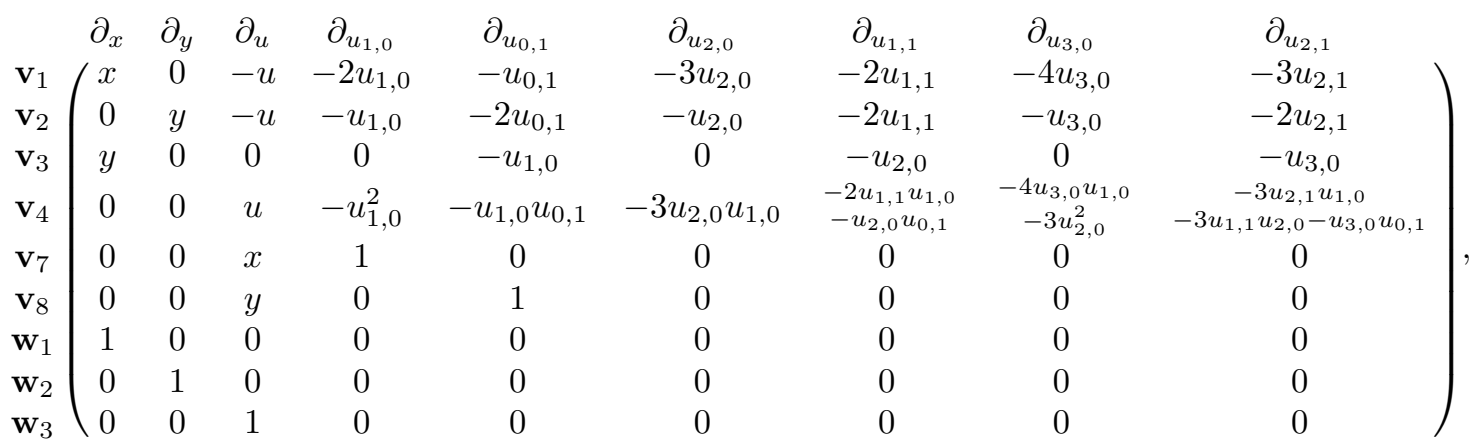

and by realizing that the upper-right $4 \times 4$ block has a not identically zero determinant:

$$
\left|\begin{array}{cccc}
-3 u_{2,0} & -2 u_{1,1} & -4 u_{3,0} & -3 u_{2,1} \\
-u_{2,0} & -2 u_{1,1} & -u_{3,0} & -2 u_{2,1} \\
0 & -u_{2,0} & 0 & -u_{3,0} \\
-3 u_{2,0} u_{1,0} & -2 u_{1,1} u_{1,0} & -4 u_{3,0} u_{1,0} & -3 u_{2,1} u_{1,0} \\
& -u_{2,0} u_{0,1} & -3 u_{2,0}^{2} & -3 u_{1,1} u_{2,0}-u_{3,0} u_{0,1}
\end{array}\right|=9 u_{2,0}^{3}(\underbrace{u_{2,0} u_{2,1}-u_{1,1} u_{3,0}}_{\text {recognize } S_{u}}) .
$$

Our domain $\left\{u_{2,0} \neq 0=u_{2,0} u_{0,2}-u_{1,1}^{2}\right\}$ stratifies as:

$$
\left\{\boldsymbol{S}_{u} \equiv 0\right\} \bigcup\left\{\boldsymbol{S}_{u} \neq 0\right\} .
$$


So on the dense open subset where $S_{u} \neq 0$, the above 9 vector fields span the tangent space to the 9-dimensional parabolic jet space $P J_{2,1}^{3}$, whence any transversal to the orbits of the (prolonged) $\mathrm{SA}_{3}$-action is zero-dimensional, and there are no differential invariants at a generic point.

Be careful! The closed subset $\left\{S_{u}=0\right\}$ is $S_{2}$-invariant. In it, the rank in question degenerates, since the above determinant has value 0 . But since $u_{2,0} \neq 0$, the equation $S_{u}=0$ can be solved for:

$$
u_{2,1}=\frac{u_{1,1}}{u_{2,0}} u_{3,0}
$$

According to Lie's general principle of thought, in the study of graphs $\{u=F(x, y)\}$, there is bifurcation branching:

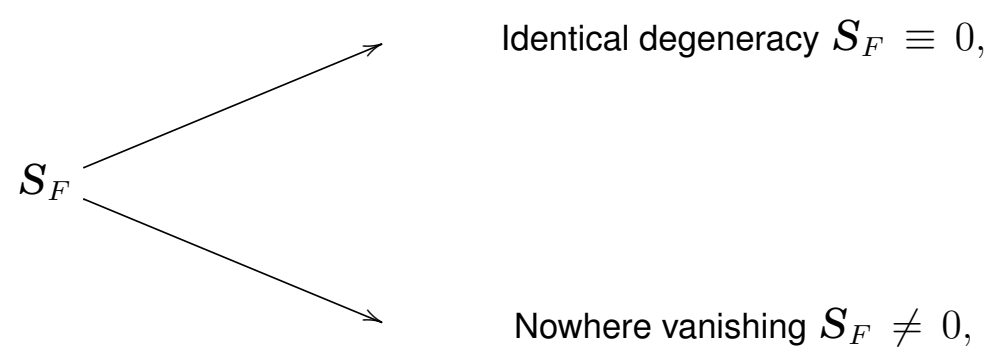

namely one studies either graphs for which $F_{x x y} \equiv \frac{F_{x y}}{F_{x x}} F_{x x x}$, or graphes for which $F_{x x} F_{x x y}-F_{x y} F_{x x x} \neq 0$ at every point $(x, y)$. In other words, we disregard mixed types for which $S_{F}(x, y) \not \equiv 0$, while $\left\{S_{F}(x, y)=0\right\} \neq \emptyset$.

Our main study, in the next Sections 18, 19, 20, will concern the branch $S_{F} \neq 0$. For now, let us summarize how the branch $S_{F} \equiv 0$ can be easily terminated.

So our assumptions are:

$$
F_{x x} \neq 0, \quad F_{y y} \equiv \frac{F_{x y}^{2}}{F_{x x}}, \quad F_{x x y} \equiv \frac{F_{x y}}{F_{x x}} F_{x x x} .
$$

This leads to a new (smooth) submanifold of the $n^{\text {th }}$ order jet space:

$$
C P J_{2,1}^{n} \subset P J_{2,1}^{n} \subset J_{2,1}^{n},
$$

defined by the equations:

$$
u_{2,0} \neq 0, \quad u_{0,2}=\frac{u_{1,1}^{2}}{u_{2,0}}, \quad u_{2,1}=\frac{u_{1,1}}{u_{2,0}} u_{3,0},
$$

together with their differential consequences (exercise).

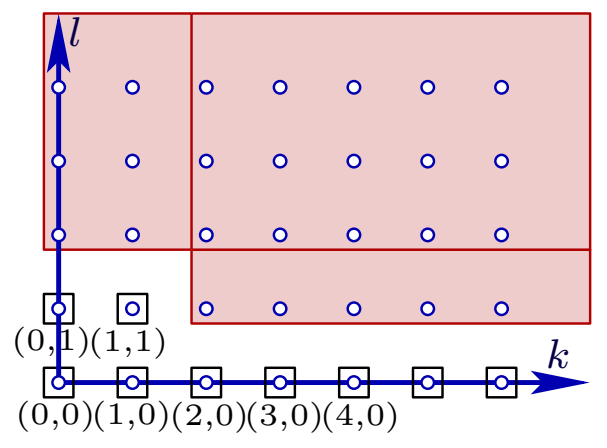


When $n \geqslant 2$, this submanifold is equipped with horizontal coordinates:

$$
C P J_{2,1}^{n}=\mathbb{R}^{5+n} \ni\left(x, y, \quad \begin{array}{c}
u_{0,1}, u_{1,1}, \\
u, \quad u_{1,0}, u_{2,0}, u_{3,0}, u_{4,0}, u_{5,0}, \ldots, u_{n, 0}
\end{array}\right) .
$$

After an elementary affine transformation, we can assume that a graphed surface satisfying these assumptions starts at order 2 terms:

$$
u=F_{2,0} \frac{x^{2}}{2 !}+F_{1,1} x y+F_{0,2} \frac{y^{2}}{2 !}+\mathrm{O}_{x, y}(3) .
$$

Since $F_{0,2}=F_{1,1}^{2} / F_{2,0}$, we can write:

$$
u=\frac{F_{2,0}}{2}\left(x+\frac{F_{1,1}}{F_{2,0}} y\right)^{2}+\mathrm{O}_{x, y}(3),
$$

and making the special affine change of coordinates:

$$
x^{\prime}:=x+\frac{F_{1,1}}{F_{2,0}} y, \quad \quad y^{\prime}:=F_{2,0} y, \quad u^{\prime}:=\frac{1}{F_{2,0}} u,
$$

we come, dropping the primes, to a normalized form:

$$
u=\frac{x^{2}}{2 !}+\mathrm{O}_{x, y}(3) \text {. }
$$

Importantly, our assumptions (17.13) are (special) affinely invariants, and then, in the new system of coordinates, they continue to hold. As in the proof of Lemma 16.8, let us write the two PDEs in question as:

$$
F_{y y}=\mathscr{R} F_{x y}, \quad F_{x x y}=\mathscr{R} F_{x y}
$$

where $\mathscr{R}=\mathscr{R}(x, y)$ denotes an unspecified function.

Assertion 17.14. In the normalized form, the remainder $\mathrm{O}_{x, y}(3)=\mathrm{O}_{x}(3)$ depends only on $x$, not on $y$.

Proof. At first, we see $F_{x x y}(0)=0$ since $F_{x y}(0)=0$. Differentiating with respect to $x$, we get:

$$
F_{x x y y}=\mathscr{R}_{x} F_{x y}+\mathscr{R} F_{x x y}=\mathscr{R} F_{x y}
$$

whence similarly $F_{x x x y}(0)=0$. An easy induction yields $F_{x^{k} y}=\mathscr{R} F_{x y}$ for every $k \geqslant 2$, whence $F_{x^{k} y}(0)=0$.

Next, we see $F_{y y}(0)=0$. Differentiating and inducting, we get $F_{x^{k} y y}=\mathscr{R} F_{x y}$ for every $k \geqslant 0$, whence $F_{x^{k} y y}(0)=0$. By another induction, $F_{x^{k} y^{l}}=\mathscr{R} F_{x y}$ for all $k \geqslant 0$, $l \geqslant 2$, whence $F_{x^{k} y^{l}}(0)=0$, and this concludes because we assume analyticity.

So surfaces $S^{2} \subset \mathbb{R}^{3}$ satisfying assumptions 17.13 are of the form:

$$
u=f(x)=\frac{x^{2}}{2 !}+\mathrm{O}_{x}(3),
$$

they are products of curves in $\mathbb{R}_{x, u}^{2}$ and the straight $\mathbb{R}_{y}^{1}$, which is a degenerate situation.

From any special affine equivalence in $\mathrm{SA}_{3}(\mathbb{R})$ we can extract a 2 -dimensional equivalence:

$$
\begin{aligned}
& s=\mathrm{a} x+\mathrm{b} y+\mathrm{c} u+\mathrm{d}, \quad s=\mathrm{a} x+\mathrm{c} u+\mathrm{d},
\end{aligned}
$$

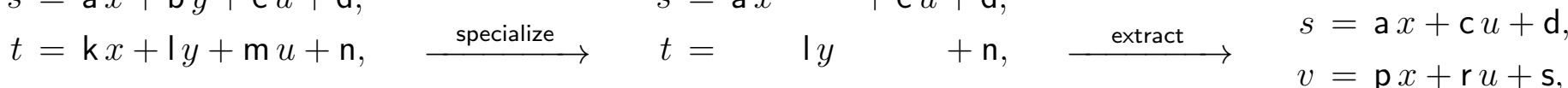

$$
\begin{aligned}
& v=\mathrm{p} x+\mathrm{q} y+\mathrm{r} u+\mathrm{s} \quad v=\mathrm{p} x \quad+\mathrm{r} u+\mathrm{s}
\end{aligned}
$$


but then, since the transformation in the $y$-space has zero influence, we can adjust the central parameter:

$$
1:=\frac{1}{\mathrm{ar}-\mathrm{cp}}
$$

in order to guarantee that the determinant has value 1 , while the determinant of the 2dimensional transformation:

$$
\left|\begin{array}{ll}
\mathrm{a} & \mathrm{c} \\
\mathrm{p} & \mathrm{r}
\end{array}\right| \neq 0
$$

must only be nonzero, not necessarily equal to 1 . In other words, our curves $\{u=f(x)\}$ with $f_{x x} \neq 0$ are considered modulo affine transformations of the plane $\mathbb{R}_{x, u}^{2}$, not special affine ones.

We have already studied plane curves modulo $A_{2}(\mathbb{R})$ in Section 16 . Hence we only need to adapt (slightly) these results in our present context.

- There is a relative invariant:

$$
P:=-5 u_{3,0}^{2}+3 u_{2,0} u_{4,0},
$$

which creates a branching.

- One has $P \equiv 0$ if and only if the surface $\{u=F(x, y)\}$ is special affinely equivalent to the product $\left\{v=s^{2}\right\}$ of a parabola with $\mathbb{R}_{t}^{1}$.

- When $P \neq 0$, as in Theorem 16.10, the surface is $\mathrm{SA}_{3}$-equivalent to:

$$
u=\frac{x^{2}}{2 !}+0 \pm \frac{x^{4}}{4 !}+F_{5} \frac{x^{5}}{5 !}+\sum_{i \geqslant 6} F_{i} \frac{x^{i}}{i !}
$$

where $F_{5}, F_{6}, F_{7}, \ldots$ are differential invariants, for instance in the case of $+\frac{x^{4}}{4 !}$ :

$$
F_{5}=I_{5}:=\frac{1}{\sqrt{3}} \frac{9 u_{2,0}^{2} u_{5,0}-45 u_{2,0} u_{3,0} u_{4,0}+40 u_{3,0}^{3}}{\left(3 u_{2,0} u_{4,0}-5 u_{3,0}^{3}\right)^{3 / 2}} .
$$

All the other differential invariants $I_{6}, I_{7}, \ldots$ express in terms of $I_{5}$ and its invariant derivatives $\mathscr{D}_{x}^{\nu}\left(I_{5}\right)$.

- The degenerate case where $I_{5} \equiv 0$ means, according to Lemma 13.10 , that there exist constants such that:

$$
u=\mathrm{d} x+\mathrm{e}+\sqrt{\mathrm{f} x^{2}+2 \mathrm{~g} x+\mathrm{h}} .
$$

There are unique such constants so that the right-hand side has fourth-order terms normalized as above:

$$
\begin{aligned}
u & =3-\sqrt{9-3 x^{2}}, & u & =-3+\sqrt{9+3 x^{2}} \\
& =\frac{x^{2}}{2 !}+0+\frac{x^{4}}{4 !}+0+\mathrm{O}_{x}(6), & & =\frac{x^{2}}{2 !}+0-\frac{x^{4}}{4 !}+0+\mathrm{O}_{x}(6) .
\end{aligned}
$$

All this study justifies that we can from now on and until the end of the paper assume that at every point $(x, y)$ :

$$
0 \neq S_{F}=F_{x x} F_{x x y}-F_{x y} F_{x x x} .
$$

Question 17.15. Are there differential invariants of order 4 ? 
Yes, at last! Remind that we already pointed out the coincidence:

$$
\operatorname{dim} P J_{2,1}^{4}=11=\operatorname{dim} \mathrm{SA}_{3}(\mathbb{R}) .
$$

One could expect that the 11 vector fields prolonged to $J_{2,1}^{4}$ and tangent to the submanifold $P J_{2,1}^{4}$ of parabolic jets:

$$
\begin{aligned}
& \mathrm{pj}_{*}\left(\mathbf{v}_{1}^{(4)}\right), \mathrm{pj}_{*}\left(\mathbf{v}_{2}^{(4)}\right), \mathrm{pj}_{*}\left(\mathbf{v}_{3}^{(4)}\right), \mathrm{pj}_{*}\left(\mathbf{v}_{4}^{(4)}\right), \mathrm{pj}_{*}\left(\mathbf{v}_{5}^{(4)}\right), \mathrm{pj}_{*}\left(\mathbf{v}_{6}^{(4)}\right), \\
& \operatorname{pj}_{*}\left(\mathbf{v}_{7}^{(4)}\right), \mathrm{pj}_{*}\left(\mathbf{v}_{8}^{(4)}\right), \mathrm{pj}_{*}\left(\mathbf{w}_{1}^{(4)}\right), \mathrm{pj}_{*}\left(\mathbf{w}_{2}^{(4)}\right), \mathrm{pj}_{*}\left(\mathbf{w}_{3}^{(4)}\right),
\end{aligned}
$$

are linearly independent at a generic point, hence span the tangent space to $P J_{2,1}^{4}$ almost everywhere, but this is not the case.

Indeed, thanks to our earlier observation (17.2) that the last 5 vector fields have identically zero coefficients in front of all the $\partial / \partial u_{j, k}$ with $j+k \geqslant 2$, it suffices to examine the rank of the first 6 vector fields in the space of jets of order $\geqslant 2$, namely, it suffices to determine the rank at a generic point of the following matrix of coefficients:

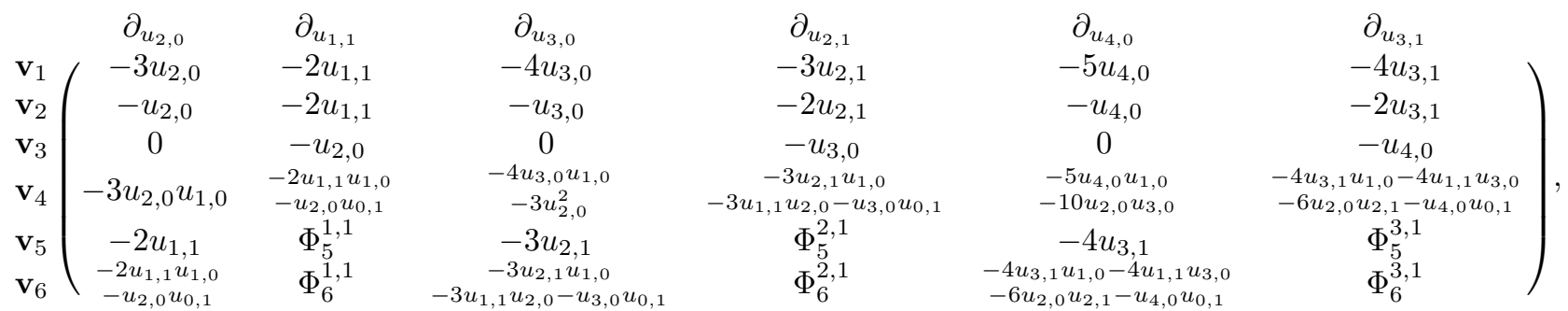

in which precisely 6 entries require take account of the parabolic jet relations shown at the end of Section 10 :

$$
\begin{aligned}
\Phi_{5}^{1,1} & =-\frac{u_{1,1}^{2}}{u_{2,0}} \\
\Phi_{5}^{2,1} & =-4 \frac{u_{1,1} u_{2,1}}{u_{2,0}}+2 \frac{u_{1,1}^{2} u_{3,0}}{u_{2,0}^{2}} \\
\Phi_{5}^{3,1} & =-6 \frac{u_{1,1} u_{3,1}}{u_{2,0}}+12 \frac{u_{3,0} u_{1,1} u_{2,1}}{u_{2,0}^{2}}-6 \frac{u_{3,0}^{2} u_{1,1}^{2}}{u_{2,0}^{3}}-6 \frac{u_{2,1}^{2}}{u_{2,0}}+3 \frac{u_{4,0} u_{1,1}^{2}}{u_{2,0}^{2}},
\end{aligned}
$$

and where:

$$
\begin{aligned}
\Phi_{6}^{1,1}= & -\frac{u_{1,0} u_{1,1}^{2}}{u_{2,0}}-2 u_{1,1} u_{0,1}, \\
\Phi_{6}^{2,1}= & -4 \frac{u_{1,0} u_{1,1} u_{2,1}}{u_{2,0}}+2 \frac{u_{1,0} u_{1,1}^{2} u_{3,0}}{u_{2,0}^{2}}-3 u_{1,1}^{2}-2 u_{2,1} u_{0,1}, \\
\Phi_{6}^{3,1}= & -6 \frac{u_{1,0} u_{1,1} u_{3,1}}{u_{2,0}}+12 \frac{u_{1,0} u_{3,0} u_{1,1} u_{2,1}}{u_{2,0}^{2}}-6 \frac{u_{1,0} u_{3,0}^{2} u_{1,1}^{2}}{u_{2,0}^{3}}-6 \frac{u_{1,0} u_{2,1}^{2}}{u_{2,0}}+ \\
& +3 \frac{u_{1,0} u_{4,0} u_{1,1}^{2}}{u_{2,0}^{2}}-12 u_{1,1} u_{2,1}+2 \frac{u_{3,0} u_{1,1}^{2}}{u_{2,0}}-2 u_{3,1} u_{0,1} .
\end{aligned}
$$

Assertion 17.16. [On a computer] The determinant of the above $6 \times 6$ matrix vanishes identically.

Question 17.17. Then what is the dimension of the generic orbits? 
Assertion 17.18. [On a computer] The above $6 \times 6$ matrix has rank 5 at every point in the domain of $P J_{2,1}^{4}$ defined by:

$$
u_{2,0} \neq 0, \quad u_{2,0} u_{2,1}-u_{1,1} u_{3,0} \neq 0 .
$$

Proof. Denote by $M^{i, j}$ the $5 \times 5$ submatrix of the above $6 \times 6$ matrix for which the $i^{\text {th }}$ row and the $j^{\text {th }}$ column are deleted. A computer gives:

$$
\begin{aligned}
\operatorname{det} M^{4,6}= & -18 u_{2,0}\left(u_{2,0} u_{2,1}-u_{1,1} u_{3,0}\right) \\
& \left(3 u_{2,0}^{2} u_{2,1}^{2}-u_{1,1} u_{2,0} u_{2,1} u_{3,0}-2 u_{1,1}^{2} u_{3,0}^{2}-2 u_{1,1} u_{2,0}^{2} u_{3,1}+2 u_{1,1}^{2} u_{2,0} u_{4,0}\right), \\
\operatorname{det} M^{5,6}= & -18 u_{2,0}\left(u_{2,0} u_{2,1}-u_{1,1} u_{3,0}\right) \\
& \left(3 u_{2,0}^{3} u_{2,1}-3 u_{1,1} u_{2,0}^{2} u_{3,0}-5 u_{1,0} u_{2,0} u_{2,1} u_{3,0}+5 u_{1,0} u_{1,1} u_{3,0}^{2}\right. \\
& \left.+2 u_{1,0} u_{2,0}^{2} u_{3,1}-2 u_{1,1}^{2} u_{2,0} u_{4,0}\right) \\
\operatorname{det} M^{6,6}= & -18 u_{2,0}\left(u_{2,0} u_{2,1}-u_{1,1} u_{3,0}\right) \\
& \left(-5 u_{2,0} u_{2,1} u_{3,0}+5 u_{1,1} u_{3,0}^{2}+2 u_{2,0}^{2} u_{3,1}-2 u_{1,1} u_{2,0} u_{4,0}\right) .
\end{aligned}
$$

Suppose these three determinants are simultaneously 0 . Then in our domain $u_{2,0} \neq 0 \neq$ $u_{2,0} u_{2,1}-u_{1,1} u_{3,0}$ we have:

$$
\begin{aligned}
& 0=3 u_{2,0}^{2} u_{2,1}^{2}-u_{1,1} u_{2,0} u_{2,1} u_{3,0}-2 u_{1,1}^{2} u_{3,0}^{2}-2 u_{1,1} u_{2,0}^{2} u_{3,1}+2 u_{1,1}^{2} u_{2,0} u_{4,0}, \\
& 0=3 u_{2,0}^{3} u_{2,1}-3 u_{1,1} u_{2,0}^{2} u_{3,0}-5 u_{1,0} u_{2,0} u_{2,1} u_{3,0}+5 u_{1,0} u_{1,1} u_{3,0}^{2}+2 u_{1,0} u_{2,0}^{2} u_{3,1}-2 u_{1,1}^{2} u_{2,0} u_{4,0}, \\
& 0=-5 u_{2,0} u_{2,1} u_{3,0}+5 u_{1,1} u_{3,0}^{2}+2 u_{2,0}^{2} u_{3,1}-2 u_{1,1} u_{2,0} u_{4,0} .
\end{aligned}
$$

Abbreviating $A:=u_{2,0} u_{2,1}-u_{1,1} u_{3,0}$ and $B:=u_{2,0} u_{3,1}-u_{1,1} u_{4,0}$, this is:

$$
\begin{aligned}
& 0=\left(3 u_{2,0} u_{2,1}+2 u_{1,1} u_{3,0}\right) A-2 u_{1,1} u_{2,0} B, \\
& 0=\left(3 u_{2,0}^{2}-5 u_{1,0} u_{3,0}\right) A+2 u_{1,0} u_{2,0} B, \\
& 0=-5 u_{3,0} A+2 u_{2,0} B .
\end{aligned}
$$

One can eliminate $u_{2,0} B$ and get:

$$
0=3 A^{2}, \quad 0=3 u_{2,0}^{2} A .
$$

These equations clearly have no solution in our domain. So the three minors cannot degenerate simultaneously, although each one can degenerate somewhere.

Consequently, there is one and only $1=6-5$ invariant. On a computer, one can solve the system of 11 PDEs:

$$
0 \equiv \mathrm{pj}_{*}\left(\mathbf{v}_{\sigma}^{(4)}\right)(W) \equiv \mathrm{pj}_{*}\left(\mathbf{w}_{\tau}^{(4)}\right)(W) \quad(1 \leqslant \sigma \leqslant 8,1 \leqslant \tau \leqslant 3),
$$

for an unknown function $W$ of the 11 jet variables:

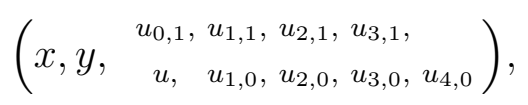

and obtain its explicit expression.

Proposition 17.19. [On a computer] This invariant is:

$$
W_{F}:=\frac{F_{x x}^{2} F_{x x x y}-F_{x x} F_{x y} F_{x x x x}+2 F_{x y} F_{x x x}^{2}-2 F_{x x} F_{x x x} F_{x x y}}{\left(F_{x x}\right)^{2}\left(F_{x x} F_{x x y}-F_{x y} F_{x x x}\right)^{2 / 3}} .
$$


Because computers rarely succeed as soon as either the number of variables or the degree increases, it would be better to have a method for computing $W$ which would also apply to determine the explicit expressions of some higher order invariants. To this aim are devoted the next two Sections $\mathbf{1 8}, 19$.

\section{Relative Invariant $S$ and First Invariant $W$}

As in Section $\mathbf{1 3}$ for the case of curves, we apply the power series method presented in Section 12 to study equivalences of analytic parabolic surfaces $S^{2} \subset \mathbb{R}^{3}$ centered at the origin:

$$
u=\sum_{j+k \geqslant 2} F_{j, k} \frac{x^{j}}{j !} \frac{y^{k}}{k !},
$$

under the action of the group $\mathrm{SL}_{3}(\mathbb{R})$ of special linear transformations. Because the special affine group $\mathrm{SA}_{3}(\mathbb{R})$ contains all translations, Theorem 12.3 already justified that the computation at the origin of the power series $\mathrm{SL}_{3}$-invariants, in the sense of Definition 12.1, is sufficient to know the differential $\mathrm{SA}_{3}$-invariants at every nearby point $(x, y)$.

Furthermore, because $\mathrm{SL}_{3}(\mathbb{R})$ also contains all vertical transvections, Theorem 12.6 already justified that we may assume the first order terms $F_{0,0}+F_{1,0} x+F_{0,1} y=0$ are already normalized to zero, as was written above.

We can therefore apply the progressive cross-section method of Section 13 . The formal coefficients $F_{j, k}$ will be re-initialized in later stages of the process. At the beginning, we assign them to be functional jets:

$$
F_{j, k}:=u_{j, k},
$$

having in mind $u_{x^{j} y^{k}}(x, y)$ in order to receive true differential invariants - but all computations will be performed at the origin. We consider special affine transformations:

$$
\mathbb{R}_{x, y, u}^{3} \longrightarrow \mathbb{R}_{s, t, v}^{3}
$$

which are not too far from the identity, so that any analytic graph $\{u=F(x, y)\}$ is sent to a similar graphed surface $\{v=G(s, t)\}$.

Since the source power series $F(x, y)=\sum F_{j, k} \frac{x^{j} y^{k}}{j ! k !}$ is given and since we want to simplify the target power series $G(s, t)=\sum G_{l, m} \frac{s^{l} t^{m}}{l ! m !}$, it is more natural to work with the inverse special affine transformation:

$$
\begin{aligned}
& x=\mathrm{a} s+\mathrm{b} t+\mathrm{c} v, \\
& y=\mathrm{k} s+\mathrm{I} t+\mathrm{m} v, \\
& u=\mathrm{p} s+\mathrm{q} t+\mathrm{r} v .
\end{aligned}
$$

Then the graphing function $G(s, t)$ is uniquely determined, by a fundamental equation:

$$
0 \equiv-\mathrm{p} s-\mathrm{q} t-\mathrm{r} v+\left.F(\mathrm{a} s+\mathrm{b} t+\mathrm{c} v, \mathrm{k} s+\mathrm{I} t+\mathrm{m} v)\right|_{\text {replace } v=G(s, t)},
$$

which holds identically as a power series in the two horizontal variables $(s, t)$. 
The goal is to use the group parameters freedom a, b, c, k, l, m, p, q, r, in order to normalize as much as possible coefficients $G_{l, m}$.

18.1. First loop. After an affine transformation, we may of course assume that our target graph enjoys a similar first-order normalization:

$$
v=\sum_{l+m \geqslant 2} G_{l, m} \frac{s^{l}}{l !} \frac{t^{m}}{m !}
$$

Then we perform the replacement:

$$
0 \equiv-\mathrm{p} s-\mathrm{q} t-\mathrm{r} G(s, t)+F(\mathrm{a} s+\mathrm{b} t+\mathrm{c} G(s, t), \mathrm{k} s+\mathrm{l} t+\mathrm{m} G(s, t)),
$$

and we glean first-order terms which must vanish:

$$
0 \equiv-\mathrm{p} s-\mathrm{q} t+\mathrm{O}_{s, t}(2) \text {. }
$$

Lemma 18.3. The subgroup of $\mathrm{SL}_{3}(\mathbb{R})$ sending $v=\mathrm{O}_{s, t}(2)$ to $u=\mathrm{O}_{x, y}(2)$ is 6 dimensional and consists of matrices:

$$
G_{\mathrm{stab}}^{(1)}: \quad\left(\begin{array}{ccc}
\mathrm{a} & \mathrm{b} & \mathrm{c} \\
\mathrm{k} & \mathrm{l} & \mathrm{m} \\
0 & 0 & \mathrm{r}
\end{array}\right)
$$

with $1=(\mathrm{al}-\mathrm{bk}) \mathrm{r}$.

Next, second order terms in (18.2) are:

$$
\begin{aligned}
0 \equiv & \left(\mathrm{k}^{2} \frac{F_{1,1}^{2}}{F_{2,0}}+\mathrm{a}^{2} F_{2,0}+2 \mathrm{ak} F_{1,1}-\mathrm{r} G_{2,0}\right) \frac{s^{2}}{2}+ \\
& +\left(\mathrm{ab} F_{2,0}+\mathrm{bk} F_{1,1}+\mathrm{al} F_{1,1}-\mathrm{r} G_{1,1}+\mathrm{kl} \frac{F_{1,1}^{2}}{F_{2,0}}\right) s t+(*) t^{2}+\mathrm{O}_{s, t}(3),
\end{aligned}
$$

where $(*)$ is unimportant. We can normalize $G_{2,0}:=1$ and $G_{1,1}:=0$ with the choice of the simple transformation:

$$
\left(\begin{array}{ccc}
\frac{1}{F_{2,0}^{1 / 3}} & -\frac{F_{1,1}}{F_{2,0}} & 0 \\
0 & 1 & 0 \\
0 & 0 & F_{2,0}^{1 / 3}
\end{array}\right) \in G_{\text {stab }}^{(1)}
$$

and the parabolic jet relation $u_{0,2}=\frac{u_{1,1}^{2}}{u_{2,0}}$ satisfied by $G(s, t)$ gives:

$$
G_{0,2}=0 \text {. }
$$

After these normalizations, third order terms become:

$0 \equiv\left(-F_{2,0}^{1 / 3} G_{3,0}+\frac{F_{3,0}}{F_{2,0}}\right) \frac{s^{3}}{6}+\left(-F_{2,0}^{1 / 3} G_{2,1}-\frac{F_{3,0} F_{1,1}}{F_{2,0}^{5 / 3}}+\frac{F_{2,1}}{F_{2,0}^{2 / 3}}\right) \frac{s^{2} t}{2}+(*) s t^{2}+(*) t^{3}+\mathrm{O}_{s, t}(4)$.

We solve for the $G_{l, m}$ and we come back to the initial functional jets:

$$
\begin{aligned}
G_{3,0} & =\frac{F_{3,0}}{F_{2,0}^{4 / 3}}=\frac{u_{3,0}}{u_{2,0}^{4 / 3}}, & G_{2,1} & =\frac{F_{2,0} F_{2,1}-F_{1,1} F_{3,0}}{F_{2,0}^{2}}=\frac{u_{2,0} u_{2,1}-u_{1,1} u_{3,0}}{u_{2,0}^{2}} \\
G_{4,0} & =\frac{F_{4,0}}{F_{2,0}^{5 / 3}}=\frac{u_{4,0}}{u_{2,0}^{5 / 3}}, & G_{3,1} & =\frac{F_{2,0} F_{3,1}-F_{1,1} F_{4,0}}{F_{2,0}^{7 / 3}}=\frac{u_{2,0} u_{3,1}-u_{1,1} u_{4,0}}{u_{2,0}^{7 / 3}} .
\end{aligned}
$$


18.4. Second loop. We restart with two formal power series normalized up to order 2 :

$$
u=\frac{x^{2}}{2 !}+\sum_{j+k \geqslant 3} F_{j, k} \frac{x^{j}}{j !} \frac{y^{k}}{k !} \quad \text { and } \quad v=\frac{s^{2}}{2 !}+\sum_{l+m \geqslant 3} G_{l, m} \frac{s^{l}}{l !} \frac{t^{m}}{m !},
$$

and with the previous stabilizer subgroup $G_{\text {stab }}^{(1)}$. The fundamental equation 18.2 ) is:

$$
\begin{aligned}
0 & \equiv-\mathrm{r} G(s, t)+F(\mathrm{a} s+\mathrm{b} t+\mathrm{c} G(s, t), \mathrm{k} s+\mathrm{I} t+\mathrm{m} G(s, t)) \\
& \equiv\left(-\mathrm{r}+\mathrm{a}^{2}\right) \frac{s^{2}}{2}+(\mathrm{ab}) s t+(*) \frac{t^{2}}{2}+\mathrm{O}_{s, t}(3) .
\end{aligned}
$$

Thus $r=a^{2}$, then $b=0$, and $1=$ alr, so $I=a^{-3}$.

Lemma 18.5. The subgroup $G_{\mathrm{stab}}^{(2)} \subset G_{\mathrm{stab}}^{(1)}$ which sends $v=\frac{1}{2} s^{2}+\cdots$ to $u=\frac{1}{2} x^{2}+\cdots$ consists of matrices:

$$
G_{\text {stab }}^{(2)}: \quad\left(\begin{array}{ccc}
\mathrm{a} & 0 & \mathrm{c} \\
\mathrm{k} & \mathrm{a}^{-3} & \mathrm{~m} \\
0 & 0 & \mathrm{a}^{2}
\end{array}\right)
$$

Next, third order terms are, with no stars present:

$$
\begin{aligned}
0 \equiv & \left(-\mathrm{a}^{2} G_{3,0}+3 \mathrm{a}^{2} \mathrm{k} F_{2,1}+3 \mathrm{ac}+\mathrm{a}^{3} F_{3,0}\right) \frac{s^{3}}{6}+ \\
& +\left(-\mathrm{a}^{2} G_{2,1}+\frac{1}{\mathrm{a}} F_{2,1}\right) \frac{s^{2} t}{2}+\mathrm{O}_{s, t}(4) .
\end{aligned}
$$

Solving:

$$
G_{2,1}=\frac{1}{\mathrm{a}^{3}} F_{2,1},
$$

we deduce from $a \neq 0$ an

Observation 18.6. After normalization of second order terms, the properties $F_{2,1}=0$ and $F_{2,1} \neq 0$ are $\mathrm{SL}_{3}(\mathbb{R})$-invariant.

Coming back to the initial functional jets, remind we have obtained just above in terms of functional jets:

$$
G_{2,1}=\frac{u_{2,0} u_{2,1}-u_{1,1} u_{3,0}}{u_{2,0}^{2}}
$$

hence we recognize the relative invariant $S_{u}$ already shown in 17.10 . After our normalization, $S_{u}$ becomes simply:

$$
\left.S_{u}\right|_{\substack{u_{2,0}=1 \\ u_{1,1}=0}}=\left.\frac{u_{2,0} u_{2,1}-u_{1,1} u_{3,0}}{u_{2,0}^{2}}\right|_{\substack{u_{2,0}=1 \\ u_{1,1}=0}}=u_{2,1}
$$

and this explains why we obtained the relation $G_{2,1}=F_{2,1} / \mathrm{a}^{3}$ between the plain monomials $G_{2,1}$ and $F_{2,1}$.

Recall that the branch $S_{u} \equiv 0$ has already been studied fully in Section 17. So we can assume $\boldsymbol{S}_{u}(x, y) \neq 0$ at every point $(x, y)$, and in the present context of power series invariants, this means that we can assume $F_{2,1} \neq 0 \neq G_{2,1}$. 
Then we can normalize $G_{2,1}:=1$ and $G_{3,0}:=0$ by means of the simple transformation:

$$
\left(\begin{array}{ccc}
F_{2,1}^{1 / 3} & 0 & 0 \\
-\frac{1}{3} \frac{F_{3,0}}{F_{2,1}^{2 / 3}} & \frac{1}{F_{2,1}} & 0 \\
0 & 0 & F_{2,1}^{2 / 3}
\end{array}\right) \in G_{\text {stab }}^{(2)} .
$$

Looking at terms of order 4 and solving, we obtain:

$$
\begin{aligned}
G_{4,0} & =-\frac{4}{3} \frac{F_{3,1} F_{3,0}}{F_{2,1}^{1 / 3}}+\frac{4}{3} \frac{F_{2,1} F_{3,0}^{2}}{F_{2,1}^{1 / 3}}+F_{2,1}^{2 / 3} F_{4,0}, \\
G_{3,1} & =\frac{F_{3,1}}{F_{2,1}^{2 / 3}}-2 F_{2,1}^{1 / 3} F_{3,0},
\end{aligned}
$$

hence coming back to functional jets:

$$
\begin{aligned}
G_{4,0} & =\frac{1}{3} \frac{4 u_{3,0} u_{2,0} u_{4,0} u_{1,1}-4 u_{3,0} u_{2,0}^{2} u_{3,1}+4 u_{3,0}^{2} u_{2,1} u_{2,0}-4 u_{3,0}^{3} u_{1,1}+3 u_{4,0} u_{2,0}^{2} u_{2,1}}{u_{2,0}^{4}\left(u_{2,0} u_{2,1}-u_{1,1} u_{3,0}\right)^{1 / 3}} \\
G_{3,1} & =\frac{-u_{2,0} u_{4,0} u_{1,1}+u_{2,0}^{2} u_{3,1}-2 u_{3,0} u_{2,1} u_{2,0}+2 u_{3,0}^{2} u_{1,1}}{u_{2,0}^{2}\left(u_{2,0} u_{2,1}-u_{1,1} u_{3,0}\right)^{2 / 3}} .
\end{aligned}
$$

Before continuing, we observe that from the parabolic jet relations shown in Section $\mathbf{1 0}$, it comes thanks to $G_{1,1}=0$ :

$$
G_{1,2}=0, \quad G_{0,3}=0 .
$$

18.7. Third loop. We restart with two formal power series normalized up to order 3 :

$u=\frac{x^{2}}{2}+\frac{x^{2} y}{2}+\sum_{j+k \geqslant 4} F_{j, k} \frac{x^{j}}{j !} \frac{y^{k}}{k !} \quad$ and $\quad v=\frac{s^{2}}{2}+\frac{s^{2} t}{2}+\sum_{l+m \geqslant 4} G_{l, m} \frac{s^{l}}{l !} \frac{t^{m}}{m !}$,

and with the previous stabilizer subgroup $G_{\mathrm{stab}}^{(2)}$. The fundamental equation 18.2 is:

$$
\begin{aligned}
0 & \equiv-\mathrm{a}^{2} G(s, t)+F\left(\mathrm{a} s+\mathrm{c} G(s, t), \mathrm{k} s+\mathrm{a}^{-3} t+\mathrm{m} G(s, t)\right) \\
& \equiv\left(3 \mathrm{a}^{2} \mathrm{k}+3 \mathrm{ac}\right) \frac{s^{3}}{6}+\left(-\mathrm{a}^{2}+\frac{1}{\mathrm{a}}\right) \frac{s^{2} t}{2}+\mathrm{O}_{s, t}(4) .
\end{aligned}
$$

Thus $\mathrm{a}=1$ and $\mathrm{k}=-\mathrm{c}$.

Lemma 18.8. The subgroup $G_{\mathrm{stab}}^{(3)} \subset G_{\mathrm{stab}}^{(2)}$ which sends $v=\frac{1}{2} s^{2}+\frac{s^{2} t}{2}+\cdots$ to $u=$ $\frac{1}{2} x^{2}+\frac{x^{2} y}{2}+\cdots$ consists of matrices:

$$
G_{\mathrm{stab}}^{(3)}: \quad\left(\begin{array}{ccc}
1 & 0 & \mathrm{c} \\
-\mathrm{c} & 1 & \mathrm{~m} \\
0 & 0 & 1
\end{array}\right)
$$

Next, fourth order terms are:

$$
\begin{aligned}
0 \equiv & \left(-G_{4,0}+3 c^{2}+6 \mathrm{~m}-4 c F_{3,1}+F_{4,0}\right) \frac{s^{4}}{24}+ \\
& +\left(-G_{3,1}+F_{3,1}\right) \frac{s^{3} t}{6}+\mathrm{O}_{s, t}(5) .
\end{aligned}
$$

Observation 18.9. There exists a (single) $4^{\text {th }}$ order invariant $G_{3,1}=F_{3,1}$. 
Its expression in terms of functional jets was already finalized at the end of the previous loop, and we attribute a name to this invariant:

$$
W:=\frac{u_{2,0}^{2} u_{3,1}-u_{2,0} u_{4,0} u_{1,1}+2 u_{3,0}^{2} u_{1,1}-2 u_{3,0} u_{2,1} u_{2,0}}{u_{2,0}^{2}\left(u_{2,0} u_{2,1}-u_{1,1} u_{3,0}\right)^{2 / 3}} .
$$

This confirms what has already been presented with prolonged vector fields in Section 17, cf. Proposition 17.19 .

Furthermore, we can make $G_{4,0}:=0$ with the simple transformation:

$$
\left(\begin{array}{ccc}
1 & 0 & 0 \\
0 & 1 & -\frac{1}{6} F_{4,0} \\
0 & 0 & 1
\end{array}\right) \in G_{\text {stab }}^{(3)}
$$

Looking at terms of order 5 and solving, we obtain:

$$
\begin{aligned}
G_{5,0} & =-\frac{5}{3} F_{4,0} F_{3,1}+F_{5,0}, \\
G_{4,1} & =F_{4,1}-3 F_{4,0},
\end{aligned}
$$

and coming back to functional jets:

$$
\begin{aligned}
& G_{5,0}=\frac{1}{9} \frac{1}{u_{2,0}^{6}\left(u_{2,0} u_{2,1}-u_{1,1} u_{3,0}\right)}\left\{5 u_{3,0} u_{2,0}^{2} u_{4,0}^{2} u_{1,1}^{2}-25 u_{3,0} u_{2,0}^{3} u_{4,0} u_{1,1} u_{3,1}\right. \\
& +30 u_{3,0}^{3} u_{2,0} u_{4,0} u_{1,1}^{2}+20 u_{3,0} u_{2,0}^{4} u_{3,1}^{2} \\
& \text { - } 40 u_{3,0}^{3} u_{2,1}^{2} u_{2,0}^{2}+80 u_{3,0}^{4} u_{2,1} u_{2,0} u_{1,1} \\
& -40 u_{3,0}^{5} u_{1,1}^{2}+15 u_{4,0}^{2} u_{2,0}^{3} u_{2,1} u_{1,1} \\
& \text { - } 15 u_{4,0} u_{2,0}^{4} u_{2,1} u_{3,1}+30 u_{3,0} u_{2,0}^{3} u_{4,0} u_{2,1}^{2} \\
& +9 u_{5,0} u_{2,0}^{4} u_{2,1}^{2}-3 u_{3,0} u_{2,0}^{3} u_{5,0} u_{1,1} u_{2,1} \\
& \text { - } 6 u_{3,0}^{2} u_{2,0}^{2} u_{5,0} u_{1,1}^{2}-15 u_{3,0} u_{2,0}^{4} u_{4,1} u_{2,1} \\
& \left.+15 u_{3,0}^{2} u_{2,0}^{3} u_{4,1} u_{1,1}-60 u_{3,0}^{2} u_{2,0}^{2} u_{4,0} u_{1,1} u_{2,1}\right\}, \\
& G_{4,1}=\frac{8 u_{3,0}^{2} u_{2,1} u_{2,0}-8 u_{3,0}^{3} u_{1,1}+7 u_{3,0} u_{2,0} u_{4,0} u_{1,1}-4 u_{3,0} u_{2,0}^{2} u_{3,1}-u_{2,0}^{2} u_{5,0} u_{1,1}+u_{2,0}^{3} u_{4,1}-3 u_{4,0} u_{2,0}^{2} u_{2,1}}{u_{2,0}^{4}\left(u_{2,0} u_{2,1}-u_{1,1} u_{3,0}\right)^{1 / 3}} .
\end{aligned}
$$

According to our general principles, we must take account of the branching that the invariant $W$ causes:

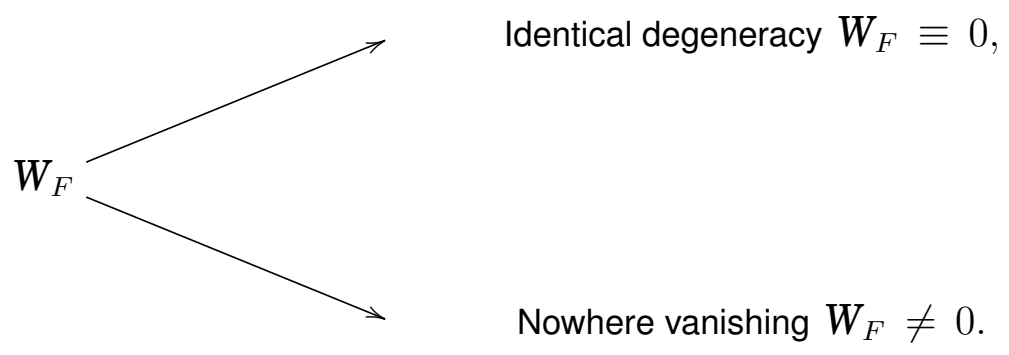

As usual, it is then natural to study first $W_{F} \equiv 0$, but before doing this in the next Section 19. we prefer to push further a bit our power series algorithm, since it will definitely show that such a branching lies inside the problem. 
Before continuing, we observe that from the parabolic jet relations shown in Section $\mathbf{1 0}$. it comes:

$$
G_{2,2}=2, \quad G_{1,3}=0, \quad G_{0,4}=0 .
$$

18.10. Fourth loop. Therefore, we restart with two formal power series normalized up to order 4:

$$
\begin{aligned}
& u=\frac{x^{2}}{2}+\frac{x^{2} y}{2}+W \frac{x^{3} y}{6}+2 \frac{x^{2} y^{2}}{4}+\sum_{j+k \geqslant 4} F_{j, k} \frac{x^{j}}{j !} \frac{y^{k}}{k !}, \\
& v=\frac{s^{2}}{2}+\frac{s^{2} t}{2}+W \frac{s^{3} t}{6}+2 \frac{s^{2} t^{2}}{4}+\sum_{l+m \geqslant 4} G_{l, m} \frac{s^{l}}{l !} \frac{t^{m}}{m !} .
\end{aligned}
$$

We repeat that for them to be $\mathrm{SA}_{3}$-equivalent, their $(3,1)$ coefficients must be equal:

$$
F_{3,1}=W=G_{3,1}
$$

We also restart with the previous stabilizer subgroup $G_{\text {stab. }}^{(3)}$ The fundamental equation 18.2 is:

$$
\begin{aligned}
0 & \equiv-G(s, t)+F(s+\mathrm{c} G(s, t),-\mathrm{c} s+t+\mathrm{m} G(s, t)) \\
& \equiv\left(6 \mathrm{~m}+3 \mathrm{c}^{2}-4 \mathrm{c} F_{3,1}\right) \frac{s^{4}}{24}+\mathrm{O}_{s, t}(5) .
\end{aligned}
$$

Lemma 18.11. The subgroup $G_{\mathrm{stab}}^{(4)} \subset G_{\mathrm{stab}}^{(3)}$ is only 1-dimensional and consists of matrices:

$$
G_{\text {stab }}^{(4)}: \quad\left(\begin{array}{ccc}
1 & 0 & c \\
-\mathrm{c} & 1 & \frac{2}{3} \mathrm{c} W-\frac{1}{2} \mathrm{c}^{2} \\
0 & 0 & 1
\end{array}\right) \text {. }
$$

Next, fifth order terms are:

$$
\begin{aligned}
0 \equiv & \left(5 c^{2} W+\frac{20}{3} c W^{2}-G_{5,0}-5 c F_{4,1}+F_{5,0}\right) \frac{s^{5}}{120}+ \\
& +\left(-G_{4,1}-2 c W+F_{4,1}\right) \frac{s^{4} t}{24}+\mathrm{O}_{s, t}(6) .
\end{aligned}
$$

Only when $W \neq 0$, we can normalize $G_{4,1}:=0$ with c $:=\frac{1}{2} \frac{F_{4,1}}{W}$, with the simple transformation:

$$
\left(\begin{array}{ccc}
1 & 0 & \frac{1}{2} \frac{F_{4,1}}{W} \\
-\frac{1}{2} \frac{F_{4,1}}{W} & 1 & \frac{1}{3} F_{4,1}-\frac{1}{8} \frac{F_{4,1}^{2}}{W^{2}} \\
0 & 0 & 1
\end{array}\right) \in G_{\text {stab }}^{(4)}
$$

The reason we normalize $G_{4,1}$ not $G_{5,0}$ when $W \neq 0$ is that the range of $G_{5,0}=5 c^{2} W+$ $\ldots$ is the range of a quadratic function of $c$. It has either a maximum or a minimum. We cannot always normalize it to 0 or any fixed real number. In other words, every orbit crosses the hyperplane $\left\{G_{4,1}=0\right\}$ exactly once, but some do not touch $\left\{G_{5,0}=0\right\}$.

This confirms our claim that the invariant $W$ causes a bifurcation, and we will now explore the two branches. 


\section{Branch $W \equiv 0$ and Branch $W \neq 0$}

Let us treat the branch $W \equiv 0$. We thus have two differential relations:

$$
\begin{aligned}
& 0 \equiv F_{x x} F_{y y}-F_{x y}^{2}, \\
& 0 \equiv F_{x x}^{2} F_{x x x y}-F_{x x} F_{x y} F_{x x x x}+2 F_{x y} F_{x x x}^{2}-2 F_{x x} F_{x x x} F_{x x y},
\end{aligned}
$$

which, thanks to the assumption $F_{x x} \neq 0$, can be solved as:

$$
\begin{aligned}
F_{y y} & =\frac{F_{x y}^{2}}{F_{x x}}, \\
F_{x x x y} & =\frac{F_{x y} F_{x x x x}}{F_{x x}}-2 \frac{F_{x y} F_{x x x}^{2}}{F_{x x}^{2}}+2 \frac{F_{x x x} F_{x x y}}{F_{x x}} .
\end{aligned}
$$

These two relations have differential consequences as well.

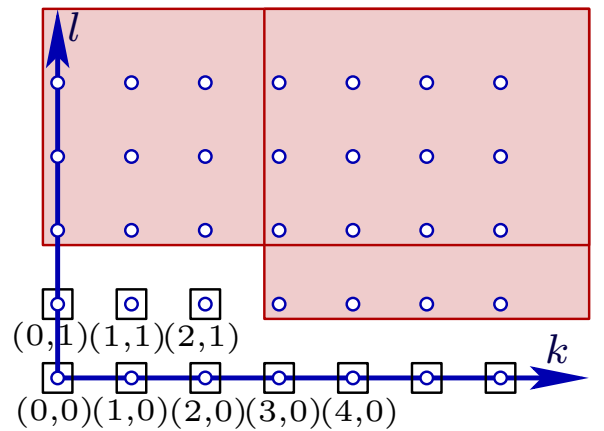

Observation 19.1. Every partial derivative $F_{x^{k} y^{l}}$ in the red region with $k+l \leqslant \mathscr{O}$ and $\mathscr{O} \geqslant 2$ expresses rationally in terms of the partial derivatives in the black region:

$$
\left\{F_{x^{k^{\prime}}}\right\}_{k^{\prime} \leqslant \mathscr{O}}, \quad\left\{F_{y}, F_{x y}, F_{x x y}\right\},
$$

with denominators containing only powers $\left(F_{x x}\right)^{*}$.

Using the jet notation, this means that we will exclusively work in the submanifold of the jet space $J_{x, u}^{\mathscr{O}}$ defined by up to order 4 by:

$$
\begin{aligned}
& F_{0,2}=\frac{F_{1,1}^{2}}{F_{2,0}}, \\
& F_{0,3}=3 \frac{F_{1,1}^{2} F_{2,1}}{F_{2,0}^{2}}-2 \frac{F_{1,1}^{3} F_{3,0}}{F_{2,0}^{3}}, \\
& F_{0,4}=12 \frac{F_{1,1}^{2} F_{2,1}^{2}}{F_{2,0}^{3}}+\frac{F_{1,1}^{4} F_{4,0}}{F_{2,0}^{4}}-16 \frac{F_{1,1}^{3} F_{3,0} F_{2,1}}{F_{2,0}^{4}}+4 \frac{F_{1,1}^{4} F_{3,0}^{2}}{F_{2,0}^{5}}, \\
& F_{1,2}=-\frac{F_{1,1}^{2} F_{3,0}}{F_{2,0}^{2}}+2 \frac{F_{1,1} F_{2,1}}{F_{2,0}}, \\
& F_{1,3}=6 \frac{F_{1,1} F_{2,1}^{2}}{F_{2,0}^{2}}+\frac{F_{1,1}^{3} F_{4,0}}{F_{2,0}^{3}}-6 \frac{F_{1,1}^{2} F_{3,0} F_{2,1}}{F_{2,0}^{3}}, \\
& F_{2,2}=\frac{F_{4,0} F_{1,1}^{2}}{F_{2,0}^{2}}-2 \frac{F_{1,1}^{2} F_{3,0}^{2}}{F_{2,0}^{3}}+2 \frac{F_{2,1}^{2}}{F_{2,0}}, \\
& F_{3,1}=\frac{F_{1,1} F_{4,0}}{F_{2,0}}-2 \frac{F_{1,1} F_{3,0}^{2}}{F_{2,0}^{2}}+2 \frac{F_{3,0} F_{2,1}}{F_{2,0}} .
\end{aligned}
$$


Observe that in contrast to the general branch $H \equiv 0 \neq W$, the jet coordinates $F_{3,1}, F_{4,1}$, ..., are dependent. Higher differential relations will also be needed, for instance:

$$
\begin{aligned}
F_{0,5}= & 60 \frac{F_{1,1}^{2} F_{2,1}^{3}}{F_{2,0}^{4}}+\frac{F_{1,1}^{5} F_{5,0}}{F_{2,0}^{5}}-120 \frac{F_{1,1}^{3} F_{2,1}^{2} F_{3,0}}{F_{2,0}^{5}}+ \\
& +15 \frac{F_{1,1}^{4} F_{4,0} F_{2,1}}{F_{2,0}^{5}}-15 \frac{F_{1,1}^{5} F_{3,0} F_{4,0}}{F_{2,0}^{6}}+60 \frac{F_{1,1}^{4} F_{2,1} F_{3,0}^{2}}{F_{2,0}^{6}}, \\
F_{1,4}= & -12 \frac{F_{1,1}^{4} F_{3,0} F_{4,0}}{F_{2,0}^{5}}+12 \frac{F_{1,1}^{4} F_{3,0}^{3}}{F_{2,0}^{6}}+12 \frac{F_{1,1}^{3} F_{2,1} F_{4,0}}{F_{2,0}^{4}}+ \\
& +\frac{F_{1,1}^{4} F_{5,0}}{F_{2,0}^{4}}+24 \frac{F_{1,1} F_{2,1}^{3}}{F_{2,0}^{3}}-36 \frac{F_{1,1}^{2} F_{3,0} F_{2,1}^{2}}{F_{2,0}^{4}}, \\
F_{2,3}= & -9 \frac{F_{3,0} F_{1,1}^{3} F_{4,0}}{F_{2,0}^{4}}-18 \frac{F_{2,1} F_{1,1}^{2} F_{3,0}^{2}}{F_{2,0}^{4}}+12 \frac{F_{3,0}^{3} F_{1,1}^{3}}{F_{2,0}^{5}}+ \\
& +9 \frac{F_{2,1} F_{1,1}^{2} F_{4,0}}{F_{2,0}^{3}}+\frac{F_{1,1}^{3} F_{5,0}}{F_{2,0}^{3}}+6 \frac{F_{2,1}^{3}}{F_{2,0}^{2}}, \\
F_{3,2}= & 6 \frac{F_{3,0}^{3} F_{1,1}^{2}}{F_{2,0}^{4}}-6 \frac{F_{3,0} F_{1,1}^{2} F_{4,0}}{F_{2,0}^{3}}+6 \frac{F_{2,1}^{2} F_{3,0}}{F_{2,0}^{2}}+ \\
& +6 \frac{F_{1,1} F_{4,0} F_{2,1}}{F_{2,0}^{2}}-12 \frac{F_{3,0}^{2} F_{1,1} F_{2,1}}{F_{2,0}^{3}}+\frac{F_{5,0} F_{1,1}^{2}}{F_{2,0}^{2}}, \\
F_{4,1}= & 3 \frac{F_{4,0} F_{2,1}}{F_{2,0}}-3 \frac{F_{3,0} F_{1,1} F_{4,0}}{F_{2,0}^{2}}+\frac{F_{1,1} F_{5,0}}{F_{2,0}} .
\end{aligned}
$$

In the branch $H \equiv 0 \equiv W$ the first three loops are the same as in the preceding Section 18. So we do not repeat the constructions.

19.2. Fourth loop. Assuming $F_{3,1}=G_{3,1}=W=0$, we restart from:

$$
\begin{aligned}
& u=\frac{x^{2}}{2}+\frac{x^{2} y}{2}+0+\frac{x^{2} y^{2}}{2}+\sum_{j+k \geqslant 5} F_{j, k} \frac{x^{j}}{j !} \frac{y^{k}}{k !}, \\
& v=\frac{s^{2}}{2}+\frac{s^{2} t}{2}+0+\frac{s^{2} t^{2}}{2}+\sum_{l+m \geqslant 5} G_{l, m} \frac{s^{l}}{l !} \frac{t^{m}}{m !} .
\end{aligned}
$$

When computing the coefficient $F_{5,0}$ in terms of the initial functional jets, we must take account of the differential relations written above, and we obtain:

$$
F_{5,0}=\frac{1}{9} \frac{\left(u_{2,0} u_{2,1}-u_{1,1} u_{3,0}\right)\left(9 u_{2,0}^{2} u_{5,0}-45 u_{2,0} u_{3,0} u_{4,0}+40 u_{3,0}^{3}\right)}{u_{2,0}^{6}} .
$$

We also see that:

$$
F_{4,1}=0, \quad F_{3,2}=0, \quad F_{2,3}=6, \quad F_{1,4}=0, \quad F_{0,5}=0 .
$$

We also restart with the group $G_{\text {stab }}^{(4)}$ of Lemma 18.11 but in which we set $W:=0$ :

$$
G_{\mathrm{stab}}^{(4)}: \quad\left(\begin{array}{ccc}
1 & 0 & \mathrm{c} \\
-\mathrm{c} & 1 & -\frac{1}{2} \mathrm{c}^{2} \\
0 & 0 & 1
\end{array}\right)
$$

This group (stabilizes) sends $v=\frac{s^{2}}{2}+\frac{s^{2} t}{2}+\frac{s^{2} t^{2}}{2}+\mathrm{O}_{s, t}(5)$ to $u=\frac{x^{2}}{2}+\frac{x^{2} y}{2}+\frac{x^{2} y^{2}}{2}+\mathrm{O}_{x, y}(5)$.

Next, we look at $5^{\text {th }}$ order terms, and we realize that:

$$
G_{5,0}=F_{5,0},
$$


hence the expression 19.3 of $F_{5,0}$ is a differential invariant. We will call it:

$$
X:=\frac{1}{9} \frac{\left(u_{x x} u_{x x y}-u_{x y} u_{x x x}\right)\left(9 u_{x x}^{2} u_{x x x x x}-45 u_{x x} u_{x x x} u_{x x x x}+40 u_{x x x}^{3}\right)}{u_{x x}^{6}} .
$$

We observe that in the current branch $u_{x x} \neq 0 \neq S$, we have:

$$
X \equiv 0 \quad \Longleftrightarrow \quad 9 u_{x x}^{2} u_{x x x x x}-45 u_{x x} u_{x x x} u_{x x x x}+40 u_{x x x}^{3} \equiv 0 \text {. }
$$

The following result has been proved for the larger, full affine group $A_{3}(\mathbb{R})$ in $[26$, Thm. 4.1]. We verify that it also holds for $\mathrm{SA}_{3}(\mathbb{R})$.

Theorem 19.4. For a local real analytic graph $\{u=F(x, y)\}$ at the origin of $\mathbb{R}_{x, y}^{2} \times \mathbb{R}_{u}^{1}$ which satisfies:

$$
F_{x x} \neq 0, \quad F_{x x} F_{y y}-F_{x y}^{2} \equiv 0, \quad F_{x x} F_{x x y}-F_{x y} F_{x x x} \neq 0,
$$

the following two conditions are equivalent:

(i) it is special affinely equivalent to:

$$
v=\frac{1}{2} \frac{s^{2}}{1-t}
$$

(ii) $W(F) \equiv 0 \equiv X(F)$, that is to say:

$$
\begin{aligned}
& 0 \equiv F_{x x}^{2} F_{x x x y}-F_{x x} F_{x y} F_{x x x x}+2 F_{x y} F_{x x x}^{2}-2 F_{x x} F_{x x x} F_{x x y}, \\
& 0 \equiv 9 F_{x x}^{2} F_{x x x x x}-45 F_{x x} F_{x x x} F_{x x x x}+40 F_{x x x}^{3} .
\end{aligned}
$$

Proof. Only (ii) $\Longrightarrow$ (i) matters. We have seen that after some $\mathrm{SA}_{3}(\mathbb{R})$ transformation, we have:

$$
u=F(x, y)=\frac{x^{2}}{2}+\frac{x^{2} y}{2}+\frac{x^{2} y^{2}}{2}+\mathrm{O}_{x, y}(5) .
$$

Because $W$ and $M$ are differential invariants, the conditions $W \equiv 0$ and $M \equiv 0$ still hold. This graphing function $F$ satisfies:

$$
\begin{aligned}
& 0=F_{x y}(0), \quad 0=F_{y y}(0), \\
& 0=F_{x x x}(0), \quad 1=F_{x x y}(0), \quad 0=F_{x y y}(0)=F_{y y y}(0), \\
& 0=F_{x x x x}(0), \quad 0=F_{x x x y}(0), \quad 2=F_{x x y y}(0), \quad 0=F_{x y y y}(0)=F_{y y y y}(0) \text {. }
\end{aligned}
$$

The condition $M(x, y) \equiv 0$, valid at every point, can be solved as:

$$
\begin{aligned}
F_{x x x x x} & =\left(5 \frac{F_{x x x x}}{F_{x x}}-\frac{40}{9} \frac{F_{x x x}^{2}}{F_{x x}^{2}}\right) F_{x x x} \\
& =\mathscr{R} \cdot F_{x x x} .
\end{aligned}
$$

We claim that $F_{x^{j}}=\mathscr{R} F_{x x x}+\mathscr{R} F_{x x x x}$ for all $j \geqslant 4$. This is true for $j=4,5$, and the induction is:

$$
\begin{aligned}
F_{x^{j+1}} & =\mathscr{R}_{x} F_{x x x}+\mathscr{R} F_{x x x x}+\mathscr{R}_{x} F_{x x x x}+\mathscr{R} F_{x x x x x} \\
& =\mathscr{R} F_{x x x}+\mathscr{R} F_{x x x x} .
\end{aligned}
$$

Since $F_{x x x}(0)=0=F_{x x x x}(0)$, we get $F_{x^{j}}(0)=0$ for all $j \geqslant 3$.

Next, we solve from $W(x, y) \equiv 0$ :

$$
\begin{aligned}
F_{x x x y} & =\left(-2 \frac{F_{x y} F_{x x x}}{F_{x x}^{2}}+2 \frac{F_{x x y}}{F_{x x}}\right) F_{x x x}+\left(\frac{F_{x y}}{F_{x x}}\right) F_{x x x x} \\
& =\mathscr{R} F_{x x x}+\mathscr{R} F_{x x x x} .
\end{aligned}
$$


The same argument gives $F_{x^{j}}(0)=0$ for all $j \geqslant 3$.

We claim that $F_{x^{j} y^{k}}(0)=0$ for all $j \geqslant 3$ and all $k \geqslant 2$. Indeed, by induction from $F_{x^{j} y^{k-1}}=\mathscr{R} F_{x x x}+\mathscr{R} F_{x x x x}$, we get:

$$
\begin{aligned}
F_{x^{j} y^{k}} & =\mathscr{R}_{y} F_{x x x}+\mathscr{R} F_{x x x y}+\mathscr{R}_{y} F_{x x x x}+\mathscr{R} F_{x x x x y} \\
& =\mathscr{R} F_{x x x}+\mathscr{R} F_{x x x x} .
\end{aligned}
$$
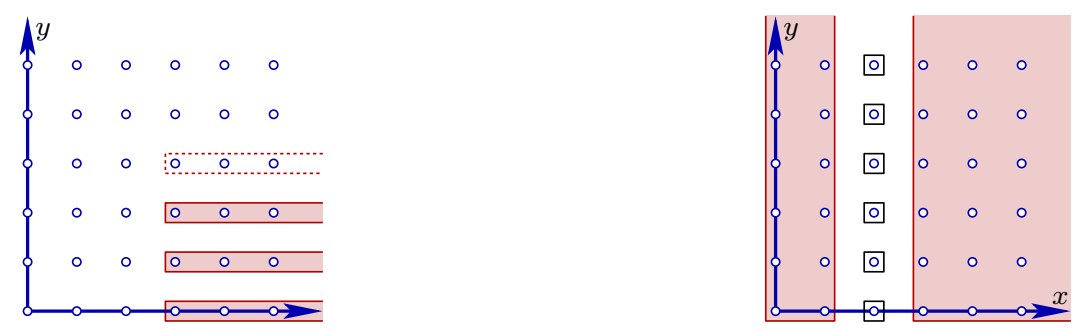

This offers $F_{x^{j} y^{k}}(0)=0$ for all $j \geqslant 3$, all all $k \geqslant 2$, and hence $F$ reduces to:

$$
F(x, y)=F_{0}(y)+x F_{1}(y)+x^{2} F_{2}(y),
$$

with $F_{2}(0)=\frac{1}{2}$, and due to the normalization above, we have $F_{0}(y)=\mathrm{O}_{y}(5)$ and $F_{1}(y)=$ $\mathrm{O}_{y}(4)$.

We can now take account of our constant hypothesis that the Hessian determinant vanishes:

$$
0 \equiv\left(2 F_{2}(y)\right)\left(F_{0, y y}+x F_{1, y y}+x^{2} F_{2, y y}\right)-\left(F_{1, y}+2 x F_{2, y}\right)^{2} .
$$

The coefficients of $x^{0}, x^{1}$ yield - remind $F_{2}(0) \neq 0$ -

$$
\begin{aligned}
& F_{0, y y}=\frac{F_{1, y}^{2}}{2 F_{2}}=\mathscr{R} F_{1, y}, \\
& F_{1, y y}=\frac{4 F_{1, y} F_{2, y}}{2 F_{2}}=\mathscr{R} F_{1, y} .
\end{aligned}
$$

From $F_{1, y}(0)=0$, we get $F_{1, y y}(0)=0$. By iteration:

$$
\begin{aligned}
F_{1, y y y} & =\mathscr{R}_{y} F_{1, y}+\mathscr{R} F_{1, y y}=\mathscr{R} F_{1, y}, \\
F_{1, y^{k}} & =\mathscr{R} F_{1, y}
\end{aligned}
$$

$(k \geqslant 3)$,

so $F_{1, y^{k}}(0)=0$, whence $F_{1}(y) \equiv 0$. It comes $F_{0, y y}(y) \equiv 0$, and lastly, $F_{0}(y) \equiv 0$. In sum:

$$
F(x, y)=x^{2} F_{2}(y)=: x^{2} T(y),
$$

with $T(0)=\frac{1}{2}=T_{y}(0)$. Back to the Hessian:

$$
0 \equiv 2 T \cdot x^{2} T_{y y}-\left(2 x T_{y}\right)^{2},
$$

we solve:

$$
\begin{aligned}
T_{y y}=2 ! \frac{\left(T_{y}\right)^{2}}{T} & \Longrightarrow \quad T_{y y y}=2 ! \frac{2 T_{y} T_{y y}}{T}-2 ! \frac{\left(T_{y}\right)^{2} T_{y}}{T^{2}}=3 ! \frac{\left(T_{y}\right)^{3}}{T^{2}} \\
& \Longrightarrow \quad T_{y^{k}}=k ! \frac{\left(T_{y}\right)^{k}}{T^{k-1}}
\end{aligned}
$$


whence $T_{y^{k}}(0)=k ! \frac{1}{2}$, and thus $T(y)=\frac{1}{2}+\frac{1}{2} y+\frac{1}{2} y^{2}+\cdots+\frac{1}{2} y^{k}+\cdots$, and finally, after having performed only special affine transformations:

$$
u=\frac{1}{2} \frac{x^{2}}{1-y} \text {. }
$$

Consequently, we can assume that $X \neq 0$.

19.5. Fifth loop. We restart from:

$$
\begin{aligned}
& u=\frac{x^{2}}{2}+\frac{x^{2} y}{2}+0+\frac{x^{2} y^{2}}{2}+X \frac{x^{5}}{5 !}+\frac{x^{2} y^{3}}{2}+\sum_{j+k \geqslant 6} F_{j, k} \frac{x^{j}}{j !} \frac{y^{k}}{k !}, \\
& v=\frac{s^{2}}{2}+\frac{s^{2} t}{2}+0+\frac{s^{2} t^{2}}{2}+X \frac{s^{5}}{5 !}+\frac{s^{2} t^{3}}{2}+\sum_{j+k \geqslant 6} G_{l, m} \frac{s^{l}}{l !} \frac{t^{m}}{m !},
\end{aligned}
$$

with a common coefficient $X \neq 0$. We examine how the 1 -dimensional subgroup $G_{\text {stab }}^{(4)}$ acts on the single independent $6^{\text {th }}$ order coefficient:

$$
\begin{aligned}
0 & \equiv-G(s, t)+F\left(\mathrm{~s}+\mathrm{c} G(s, t),-\mathrm{c} s+t-\frac{1}{2} \mathrm{c}^{2} G(s, t)\right) \\
& \equiv\left(-3 \mathrm{c} X+F_{6,0}-G_{6,0}\right) \frac{s^{6}}{6 !}+\mathrm{O}_{s, t}(7),
\end{aligned}
$$

Thus, we can normalize $G_{6,0}:=0$ by means of the simple transformation:

$$
\left(\begin{array}{ccc}
1 & 0 & \frac{1}{3} \frac{F_{6,0}}{X} \\
-\frac{1}{3} \frac{F_{6,0}}{X} & 1 & -\frac{1}{18} \frac{F_{6,0}^{2}}{X^{2}} \\
0 & 0 & 1
\end{array}\right) \in G_{\text {stab }}^{(4)} .
$$

Then in terms of functional jets, there is an invariant of order 7 , that we call $Y$ :

$$
\begin{aligned}
Y:= & \frac{1}{18} \frac{1}{u_{2,0}^{10}\left(9 u_{5,0} u_{2,0}^{2}-45 u_{3,0} u_{4,0} u_{2,0}+40 u_{3,0}^{3}\right)}\left(\left(11200 u_{3,0}^{8}-12600 u_{3,0}^{3} u_{5,0}\right.\right. \\
& u_{2,0}^{3} u_{4,0}+13230 u_{3,0} u_{5,0} u_{2,0}^{4} u_{4,0}^{2}+1134 u_{3,0} u_{5,0} u_{2,0}^{5} u_{6,0}-3150 u_{3,0}^{2} u_{4,0} u_{2,0}^{4} u_{6,0} \\
& -810 u_{7,0} u_{2,0}^{5} u_{3,0} u_{4,0}-33600 u_{3,0}^{6} u_{4,0} u_{2,0}-7875 u_{3,0}^{2} u_{4,0}^{3} u_{2,0}^{3}-756 u_{3,0}^{2} u_{5,0}^{2} u_{2,0}^{4} \\
& +6720 u_{3,0}^{5} u_{5,0} u_{2,0}^{2}+31500 u_{3,0}^{4} u_{4,0}^{2} u_{2,0}^{2}-4725 u_{4,0}^{4} u_{2,0}^{4}-189 u_{6,0}^{2} u_{2,0}^{6}+1890 u_{4,0}^{2} \\
& \left.u_{2,0}^{5} u_{6,0}-2835 u_{4,0} u_{2,0}^{5} u_{5,0}^{2}+162 u_{7,0} u_{2,0}^{6} u_{5,0}+720 u_{7,0} u_{2,0}^{4} u_{3,0}^{3}\right)\left(u_{2,1} u_{2,0}\right. \\
& \left.\left.-u_{3,0} u_{1,1}\right)^{5 / 3}\right)
\end{aligned}
$$

The synthesis for this branch is made in Section 1, especially in Theorem 2.11.

Next, we treat the branch $W \neq 0$. At the end of Section 18, we have seen that when $W \neq 0$, we can normalize $G_{4,1}=0$. Solving the other coefficient there, we get:

$$
G_{5,0}=-\frac{5}{4} \frac{F_{4,1}^{2}}{F_{3,1}}+\frac{10}{3} F_{3,1} F_{4,1}+F_{5,0} .
$$

Since the last group parameter $\mathrm{c}$ has been consumed, it is not necessary to do a fifth loop, and we conclude that:

Observation 19.6. In the branch $W \neq 0$, there is a (single) $5^{\text {th }}$ order $\mathrm{SA}_{3}$-invariant. 
In terms of functional jets, its explicit expression incorporates $\mathbf{5 7}$ monomials in the numerator, and we call it $M$.

$$
\begin{aligned}
& M:=\frac{1}{36}\left(270 u_{2,0}^{6} u_{4,1} u_{2,1}^{2} u_{4,0}-72 u_{3,0} u_{5,0} u_{2,0}^{5} u_{2,1}^{3}+820 u_{3,0} u_{2,0}^{3} u_{4,0}^{3} u_{1,1}^{3}-2195 u_{3,0}^{3}\right. \\
& u_{2,0}^{2} u_{4,0}^{2} u_{1,1}^{3}+2560 u_{3,0}^{5} u_{2,0} u_{4,0} u_{1,1}^{3}+2000 u_{3,0}^{2} u_{2,0}^{5} u_{3,1}^{2} u_{2,1}-2000 u_{3,0}^{3} u_{2,0}^{4} u_{3,1}^{2} u_{1,1} \\
& -3040 u_{3,0}^{3} u_{2,0}^{4} u_{3,1} u_{2,1}^{2}-3040 u_{3,0}^{5} u_{2,0}^{2} u_{3,1} u_{1,1}^{2}-3840 u_{3,0}^{5} u_{2,1}^{2} u_{2,0}^{2} u_{1,1}+3840 \\
& u_{3,0}^{6} u_{2,1} u_{2,0} u_{1,1}^{2}-420 u_{4,0}^{3} u_{2,0}^{4} u_{2,1} u_{1,1}^{2}+480 u_{4,0} u_{2,0}^{4} u_{2,1}^{3} u_{3,0}^{2}-420 u_{2,1} u_{2,0}^{6} u_{4,0} \\
& u_{3,1}^{2}+192 u_{3,0}^{4} u_{2,0}^{2} u_{5,0} u_{1,1}^{3}-120 u_{3,0}^{2} u_{2,0}^{5} u_{4,1} u_{2,1}^{2}-120 u_{3,0}^{4} u_{2,0}^{3} u_{4,1} u_{1,1}^{2}+36 u_{5,0} \\
& u_{2,0}^{6} u_{2,1}^{2} u_{3,1}+45 u_{2,0}^{6} u_{4,1}^{2} u_{3,0} u_{1,1}-45 u_{2,0}^{5} u_{5,0}^{2} u_{1,1}^{2} u_{2,1}+45 u_{2,0}^{4} u_{5,0}^{2} u_{1,1}^{3} u_{3,0}-120 \\
& u_{2,0}^{4} u_{5,0} u_{1,1}^{3} u_{4,0}^{2}-120 u_{2,0}^{6} u_{5,0} u_{1,1} u_{3,1}^{2}+120 u_{2,0}^{5} u_{4,1} u_{4,0}^{2} u_{1,1}^{2}+1280 u_{3,0}^{4} u_{2,1}^{3} u_{2,0}^{3} \\
& -400 u_{3,0} u_{2,0}^{6} u_{3,1}^{3}-45 u_{2,0}^{7} u_{4,1}^{2} u_{2,1}-405 u_{2,1}^{3} u_{2,0}^{5} u_{4,0}^{2}+120 u_{2,0}^{7} u_{4,1} u_{3,1}^{2}-1280 \\
& u_{3,0}^{7} u_{1,1}^{3}-5200 u_{3,0}^{2} u_{2,0}^{4} u_{4,0} u_{1,1} u_{3,1} u_{2,1}+432 u_{3,0} u_{2,0}^{4} u_{4,0} u_{1,1}^{2} u_{5,0} u_{2,1}-360 u_{3,0} \\
& u_{2,0}^{5} u_{4,0} u_{1,1} u_{4,1} u_{2,1}+108 u_{3,0} u_{2,0}^{5} u_{3,1} u_{5,0} u_{1,1} u_{2,1}-2040 u_{3,0} u_{2,0}^{4} u_{4,0}^{2} u_{1,1}^{2} u_{3,1} \\
& +1985 u_{3,0}^{2} u_{2,0}^{3} u_{4,0}^{2} u_{1,1}^{2} u_{2,1}+1620 u_{3,0} u_{2,0}^{5} u_{4,0} u_{1,1} u_{3,1}^{2}+4600 u_{3,0}^{3} u_{2,0}^{3} u_{4,0} \\
& u_{1,1}^{2} u_{3,1}+1600 u_{3,0}^{3} u_{2,0}^{3} u_{4,0} u_{1,1} u_{2,1}^{2}-4640 u_{3,0}^{4} u_{2,0}^{2} u_{4,0} u_{1,1}^{2} u_{2,1}+6080 u_{3,0}^{4} \\
& u_{2,0}^{3} u_{3,1} u_{2,1} u_{1,1}+840 u_{4,0}^{2} u_{2,0}^{5} u_{2,1} u_{1,1} u_{3,1}+615 u_{4,0}^{2} u_{2,0}^{4} u_{2,1}^{2} u_{1,1} u_{3,0}+600 u_{4,0} \\
& u_{2,0}^{5} u_{2,1}^{2} u_{3,1} u_{3,0}+336 u_{3,0}^{2} u_{2,0}^{4} u_{5,0} u_{1,1} u_{2,1}^{2}-456 u_{3,0}^{3} u_{2,0}^{3} u_{5,0} u_{1,1}^{2} u_{2,1}-126 u_{3,0}^{2} \\
& u_{2,0}^{3} u_{4,0} u_{1,1}^{3} u_{5,0}+90 u_{3,0}^{2} u_{2,0}^{4} u_{4,0} u_{1,1}^{2} u_{4,1}-144 u_{3,0}^{2} u_{2,0}^{4} u_{3,1} u_{5,0} u_{1,1}^{2}-306 \\
& u_{2,0}^{5} u_{5,0} u_{1,1} u_{4,0} u_{2,1}^{2}+240 u_{3,0}^{3} u_{2,0}^{4} u_{4,1} u_{2,1} u_{1,1}-180 u_{3,0} u_{2,0}^{6} u_{3,1} u_{4,1} u_{2,1}+180 \\
& u_{3,0}^{2} u_{2,0}^{5} u_{3,1} u_{4,1} u_{1,1}+90 u_{2,0}^{6} u_{5,0} u_{1,1} u_{4,1} u_{2,1}-90 u_{2,0}^{5} u_{5,0} u_{1,1}^{2} u_{4,1} u_{3,0}+240 \\
& \left.u_{2,0}^{5} u_{5,0} u_{1,1}^{2} u_{4,0} u_{3,1}-240 u_{2,0}^{6} u_{4,1} u_{4,0} u_{1,1} u_{3,1}\right) /\left(\left(u_{2,0} u_{4,0} u_{1,1}-u_{2,0}^{2} u_{3,1}\right.\right. \\
& \left.\left.+2 u_{3,0} u_{2,1} u_{2,0}-2 u_{3,0}^{2} u_{1,1}\right) u_{2,0}^{6}\left(-u_{2,1} u_{2,0}+u_{3,0} u_{1,1}\right)\right)
\end{aligned}
$$

The synthesis for this branch is made in Section 1 , especially in Theorem 2.12.

\section{Recurrence Relations for Parabolic Surfaces}

Since the branch $S \equiv 0$ has already been done in Section 16, we assume from now on that $S \neq 0$, still with $F_{x x} \neq 0 \equiv H$.

20.1. Branch $W \neq 0$. By what precedes, the normalization at the origin takes the form:

$$
\begin{aligned}
u & =F(x, y) \\
& =\frac{x^{2}}{2}+\frac{x^{2} y}{2}+W \frac{x^{3} y}{6}+\frac{x^{2} y^{2}}{2}+M \frac{x^{5}}{5 !}+6 W \frac{x^{3} y^{2}}{3 ! 2 !}+\frac{x^{2} y^{3}}{2}+\mathrm{O}_{x, y}(6) .
\end{aligned}
$$

Later, we will need from parabolic jet relations:

$$
F_{4,2}=6\left(F_{3,1}\right)^{2}=6 W^{2} .
$$

All invariants are:

$$
I_{j, k}:=\operatorname{inv}\left(u_{x^{j} y^{k}}\right)
$$

Of course, $\mathrm{SA}_{3}(\mathbb{R})$ contains all translations and all vertical transvections, hence Theorem 12.6 applies.

Moreover, by Section 14.9 , we can reduce ourselves to the 6 vector fields: 


$$
\begin{array}{r}
\mathbf{v}_{\mathbf{1}}:=x \partial_{x}-u \partial_{u}, \quad \mathbf{v}_{2}:=y \partial_{y}-u \partial_{u}, \\
\mathbf{v}_{3}:=y \partial_{x}, \quad \mathbf{v}_{4}:=u \partial_{x}, \quad \mathbf{v}_{5}:=x \partial_{y}, \quad \mathbf{v}_{6}:=u \partial_{y},
\end{array}
$$

and we have the recurrence formulae:

$$
\begin{aligned}
& \mathscr{D}_{1} \boldsymbol{I}_{j, k}=\boldsymbol{I}_{j+1, k}+\sum_{1 \leqslant \sigma \leqslant 6} \Phi_{\sigma}^{j, k}\left(\boldsymbol{I}^{(j+k)}\right) \cdot \boldsymbol{K}_{1}^{\sigma}, \\
& \mathscr{D}_{2} \boldsymbol{I}_{j, k}=\boldsymbol{I}_{j, k+1}+\sum_{1 \leqslant \sigma \leqslant 6} \Phi_{\sigma}^{j, k}\left(\boldsymbol{I}^{(j+k)}\right) \cdot \boldsymbol{K}_{2}^{\sigma} .
\end{aligned}
$$

According to what precedes, the 6 relevant phantom invariants are:

$$
I_{2,0}=1, \quad I_{1,1}=0, \quad I_{3,0}=0, \quad I_{2,1}=1, \quad I_{4,0}=0, \quad I_{4,1}=0 .
$$

We denote specially:

$$
W:=I_{3,1} \quad \text { and } \quad M:=I_{5,0},
$$

and we apply the first collection of recurrence formulas:

$$
\left(\begin{array}{l}
0 \\
0 \\
0 \\
0 \\
0 \\
0
\end{array}\right)=\left(\begin{array}{c}
\mathscr{D}_{1} \boldsymbol{I}_{2,0} \\
\mathscr{D}_{1} \boldsymbol{I}_{1,1} \\
\mathscr{D}_{1} \boldsymbol{I}_{3,0} \\
\mathscr{D}_{1} \boldsymbol{I}_{2,1} \\
\mathscr{D}_{1} \boldsymbol{I}_{4,0} \\
\mathscr{D}_{1} \boldsymbol{I}_{4,1}
\end{array}\right)=\left(\begin{array}{c}
1 \\
0 \\
W \\
\boldsymbol{M} \\
\boldsymbol{I}_{5,1}
\end{array}\right)+\left(\begin{array}{cccccc}
-3 & -1 & 0 & 0 & 0 & 0 \\
0 & 0 & -1 & 0 & 0 & 0 \\
0 & 0 & 0 & -3 & -3 & 0 \\
-3 & -2 & 0 & 0 & 0 & 0 \\
0 & 0 & 0 & 0 & -4 W & -6 \\
0 & 0 & -M & -10 W & -24 W & -18
\end{array}\right)\left(\begin{array}{c}
K_{1}^{1} \\
K_{1}^{2} \\
K_{1}^{3} \\
K_{1}^{4} \\
K_{1}^{5} \\
\boldsymbol{K}_{1}^{6}
\end{array}\right) .
$$

This Cramér system has the unique solution:

$$
\begin{aligned}
& K_{1}^{1}=-\frac{1}{3} W, \quad K_{1}^{2}=W, \quad K_{1}^{3}=1, \\
& K_{1}^{4}=2 \frac{M}{W}-\frac{1}{2} \frac{I_{5,1}}{W}, \quad K_{1}^{5}=-2 \frac{M}{W}+\frac{1}{2} \frac{I_{5,1}}{W}, \quad K_{1}^{6}=\frac{3}{2} M-\frac{1}{3} I_{5,1} .
\end{aligned}
$$

The second collection is:

$$
\left(\begin{array}{l}
0 \\
0 \\
0 \\
0 \\
0 \\
0
\end{array}\right)=\left(\begin{array}{c}
\mathscr{D}_{2} \boldsymbol{I}_{2,0} \\
\mathscr{D}_{2} \boldsymbol{I}_{1,1} \\
\mathscr{D}_{2} \boldsymbol{I}_{3,0} \\
\mathscr{D}_{2} \boldsymbol{I}_{2,1} \\
\mathscr{D}_{2} \boldsymbol{I}_{4,0} \\
\mathscr{D}_{2} \boldsymbol{I}_{4,1}
\end{array}\right)=\left(\begin{array}{c}
1 \\
W \\
2 \\
0 \\
6 W^{2}
\end{array}\right)+\left(\begin{array}{cccccc}
-3 & -1 & 0 & 0 & 0 & 0 \\
0 & 0 & -1 & 0 & 0 & 0 \\
0 & 0 & 0 & -3 & -3 & 0 \\
-3 & -2 & 0 & 0 & 0 & 0 \\
0 & 0 & 0 & 0 & -4 W & -6 \\
0 & 0 & -M & -10 W & -24 W & -18
\end{array}\right)\left(\begin{array}{c}
K_{2}^{1} \\
K_{2}^{2} \\
K_{2}^{3} \\
K_{2}^{4} \\
K_{2}^{5} \\
K_{2}^{6}
\end{array}\right),
$$

and has the unique solution:

$$
K_{2}^{1}=0, \quad K_{2}^{2}=1, \quad K_{2}^{3}=0, \quad K_{2}^{4}=-W, \quad K_{2}^{5}=\frac{4}{3} W, \quad K_{2}^{6}=-\frac{8}{9} W^{2} .
$$

For the three non-phantom invariants:

$$
I_{3,1}=: W, \quad I_{5,0}=: M, \quad I_{6,0},
$$


the recurrence formulae are:

$$
\left(\begin{array}{l}
\mathscr{D}_{1} \boldsymbol{W} \\
\mathscr{D}_{1} \boldsymbol{M} \\
\mathscr{D}_{1} \boldsymbol{I}_{6,0}
\end{array}\right)=\left(\begin{array}{c}
0 \\
\boldsymbol{I}_{6,0} \\
\boldsymbol{I}_{7,0}
\end{array}\right)+\left(\begin{array}{cccccc}
-4 W & -2 W & 0 & -6 & -6 & 0 \\
-6 \boldsymbol{M} & -\boldsymbol{M} & 0 & 0 & 0 & -10 \boldsymbol{W} \\
-7 \boldsymbol{I}_{6,0} & -\boldsymbol{I}_{6,0} & 0 & -21 \boldsymbol{M} & -6 \boldsymbol{I}_{5,1} & 0
\end{array}\right)\left(\begin{array}{c}
\boldsymbol{K}_{1}^{1} \\
\boldsymbol{K}_{1}^{2} \\
\boldsymbol{K}_{1}^{3} \\
\boldsymbol{K}_{1}^{4} \\
\boldsymbol{K}_{1}^{5} \\
\boldsymbol{K}_{1}^{6}
\end{array}\right)
$$

and:

$$
\left(\begin{array}{l}
\mathscr{D}_{2} W \\
\mathscr{D}_{2} \boldsymbol{M} \\
\mathscr{D}_{2} \boldsymbol{I}_{6,0}
\end{array}\right)=\left(\begin{array}{c}
6 W \\
I_{5,1} \\
\boldsymbol{I}_{6,1}
\end{array}\right)+\left(\begin{array}{cccccc}
-4 W & -2 W & 0 & -6 & -6 & 0 \\
-6 \boldsymbol{M} & -\boldsymbol{M} & 0 & 0 & 0 & -10 W \\
-7 I_{6,0} & -\boldsymbol{I}_{6,0} & 0 & -21 M & -6 \boldsymbol{I}_{5,1} & 0
\end{array}\right)\left(\begin{array}{c}
K_{2}^{1} \\
K_{2}^{2} \\
K_{2}^{3} \\
K_{2}^{4} \\
K_{2}^{5} \\
K_{2}^{6}
\end{array}\right) .
$$

So we obtain:

$$
\begin{aligned}
\mathscr{D}_{1} W & =-\frac{2 W^{2}}{3}, & \mathscr{D}_{2} W & =2 W, \\
\mathscr{D}_{1} M & =I_{6,0}-14 M W+\frac{10}{3} I_{5,1} W, & \mathscr{D}_{2} M & =I_{5,1}-M+\frac{80}{9} W^{3},
\end{aligned}
$$

and:

$$
\begin{aligned}
& \mathscr{D}_{1} \boldsymbol{I}_{6,0}=I_{7,0}-\frac{3}{2 W}\left(7 M-2 I_{5,1}\right)\left(4 M-I_{5,1}\right)+\frac{4}{3} W I_{6,0} \\
& \mathscr{D}_{2} I_{6,0}=I_{6,1}-I_{6,0}+21 W M-8 W I_{5,1} .
\end{aligned}
$$

Looking at these equations, we see that we can solve the $6^{\text {th }}$ order invariants $I_{6,0}$ and $I_{5,1}$ in terms of $W, M, \mathscr{D}_{1} M, \mathscr{D}_{2} M$. We can also solve $I_{7,0}, I_{6,1}$ in terms of $W, M$ and their invariant derivatives. An elementary induction yields:

Proposition 20.2. Within the branch $S \neq 0, W \neq 0$, all invariants are generated by $W, M$ and their invariant derivatives.

Moreover, $M$ cannot be obtained from $W$ and its invariant derivatives.

Proof. Indeed, both $\mathscr{D}_{1} W=-\frac{2}{3} W^{2}$ and $\mathscr{D}_{2} W=2 W$ do not raise the jet order.

20.3. Branch $W \equiv 0$. Thus, we assume $S \neq 0$ and $W \equiv 0$. Since the case $X \equiv 0$ has already been covered by Theorem 19.4, we may also assume $X \neq 0$.

According to Theorem 2.11, the normal form is:

$$
\begin{aligned}
u=\frac{x^{2}}{2}+\frac{x^{2} y}{2}+\frac{x^{2} y^{2}}{2}+F_{5,0} \frac{x^{5}}{120}+\frac{x^{2} y^{3}}{2}+4 F_{5,0} \frac{x^{5} y}{120}+\frac{x^{2} y^{4}}{2}+F_{7,0} \frac{x^{7}}{5040} & +20 F_{5,0} \frac{x^{5} y^{2}}{240}+\frac{x^{2} y^{5}}{2}+ \\
& +\sum_{j+k \geqslant 8} F_{j, k} x^{j} y^{k}
\end{aligned}
$$

and this means that we have 6 phantom invariants:

$$
I_{2,0}=1, \quad I_{1,1}=0, \quad I_{3,0}=0, \quad I_{2,1}=1, \quad I_{4,0}=0, \quad I_{6,0}=0 .
$$

We denote specially:

$$
X:=I_{5,0} \quad \text { and } \quad Y:=I_{7,0},
$$


and we apply the first collection of recurrence formulas:

$$
\left(\begin{array}{l}
0 \\
0 \\
0 \\
0 \\
0 \\
0
\end{array}\right)=\left(\begin{array}{l}
\mathscr{D}_{1} \boldsymbol{I}_{2,0} \\
\mathscr{D}_{1} \boldsymbol{I}_{1,1} \\
\mathscr{D}_{1} \boldsymbol{I}_{3,0} \\
\mathscr{D}_{1} \boldsymbol{I}_{2,1} \\
\mathscr{D}_{1} \boldsymbol{I}_{4,0} \\
\mathscr{D}_{1} \boldsymbol{I}_{6,0}
\end{array}\right)=\left(\begin{array}{c}
0 \\
1 \\
0 \\
0 \\
\boldsymbol{X} \\
\boldsymbol{Y}
\end{array}\right)+\left(\begin{array}{cccccc}
-3 & -1 & 0 & 0 & 0 & 0 \\
0 & 0 & -1 & 0 & 0 & 0 \\
0 & 0 & 0 & -3 & -3 & 0 \\
-3 & -2 & 0 & 0 & 0 & 0 \\
0 & 0 & 0 & 0 & 0 & -6 \\
0 & 0 & 0 & -21 X & -24 X & 0
\end{array}\right)\left(\begin{array}{c}
\boldsymbol{K}_{1}^{1} \\
\boldsymbol{K}_{1}^{2} \\
\boldsymbol{K}_{1}^{3} \\
\boldsymbol{K}_{1}^{4} \\
\boldsymbol{K}_{1}^{5} \\
\boldsymbol{K}_{1}^{6}
\end{array}\right) .
$$

This Cramér system has the unique solution:

$$
K_{1}^{1}=0, \quad K_{1}^{2}=0, \quad K_{1}^{3}=1, \quad K_{1}^{4}=-\frac{Y}{3 X}, \quad K_{1}^{5}=\frac{Y}{3 X}, \quad K_{1}^{6}=\frac{1}{6} X
$$

The second collection is:

$$
\left(\begin{array}{l}
0 \\
0 \\
0 \\
0 \\
0 \\
0
\end{array}\right)=\left(\begin{array}{l}
\mathscr{D}_{2} \boldsymbol{I}_{2,0} \\
\mathscr{D}_{2} \boldsymbol{I}_{1,1} \\
\mathscr{D}_{2} \boldsymbol{I}_{3,0} \\
\mathscr{D}_{2} \boldsymbol{I}_{2,1} \\
\mathscr{D}_{2} \boldsymbol{I}_{4,0} \\
\mathscr{D}_{2} \boldsymbol{I}_{6,0}
\end{array}\right)=\left(\begin{array}{l}
1 \\
0 \\
0 \\
2 \\
0 \\
0
\end{array}\right)+\left(\begin{array}{cccccc}
-3 & -1 & 0 & 0 & 0 & 0 \\
0 & 0 & -1 & 0 & 0 & 0 \\
0 & 0 & 0 & -3 & -3 & 0 \\
-3 & -2 & 0 & 0 & 0 & 0 \\
0 & 0 & 0 & 0 & 0 & -6 \\
0 & 0 & 0 & -21 X & -24 X & 0
\end{array}\right)\left(\begin{array}{c}
K_{2}^{1} \\
K_{2}^{2} \\
K_{2}^{3} \\
K_{2}^{4} \\
K_{2}^{5} \\
\boldsymbol{K}_{2}^{6}
\end{array}\right)
$$

and has the unique solution:

$$
K_{2}^{1}=0, \quad K_{2}^{2}=1, \quad K_{2}^{3}=0, \quad K_{2}^{4}=0, \quad K_{2}^{5}=0, \quad K_{2}^{6}=0 .
$$

For the three non-phantom invariants:

$$
I_{5,0}=: X, \quad I_{7,0}=: Y, \quad I_{8,0},
$$

the recurrence formulae are:

$$
\left(\begin{array}{c}
\mathscr{D}_{1} \boldsymbol{X} \\
\mathscr{D}_{1} \boldsymbol{Y} \\
\mathscr{D}_{1} \boldsymbol{I}_{8,0}
\end{array}\right)=\left(\begin{array}{c}
0 \\
\boldsymbol{I}_{8,0} \\
\boldsymbol{I}_{9,0}
\end{array}\right)+\left(\begin{array}{cccccc}
-6 X & -X & 0 & 0 & 0 & 0 \\
-8 Y & -Y & 0 & 0 & 0 & -105 X \\
-9 \boldsymbol{I}_{8,0} & -\boldsymbol{I}_{8,0} & 0 & -36 Y & -48 Y & 0
\end{array}\right)\left(\begin{array}{c}
K_{1}^{1} \\
K_{1}^{2} \\
K_{1}^{3} \\
K_{1}^{4} \\
K_{1}^{5} \\
K_{1}^{6}
\end{array}\right)
$$

and:

$$
\left(\begin{array}{c}
\mathscr{D}_{2} \boldsymbol{X} \\
\mathscr{D}_{2} \boldsymbol{Y} \\
\mathscr{D}_{2} \boldsymbol{I}_{8,0}
\end{array}\right)=\left(\begin{array}{c}
4 X \\
6 Y \\
7 \boldsymbol{I}_{8,0}
\end{array}\right)+\left(\begin{array}{cccccc}
-6 X & -X & 0 & 0 & 0 & 0 \\
-8 Y & -Y & 0 & 0 & 0 & -105 X \\
-9 I_{8,0} & -I_{8,0} & 0 & -36 Y & -48 Y & 0
\end{array}\right)\left(\begin{array}{c}
K_{2}^{1} \\
K_{2}^{2} \\
K_{2}^{3} \\
K_{2}^{4} \\
K_{2}^{5} \\
K_{2}^{6}
\end{array}\right) .
$$

So we obtain:

$$
\begin{array}{rlrl}
\mathscr{D}_{1} X & =0, \\
\mathscr{D}_{1} Y & =I_{8,0}-\frac{35}{2} X^{2}, & \mathscr{D}_{2} X & =3 X, \\
\mathscr{D}_{1} I_{8,0} & =I_{9,0}-4 \frac{Y^{2}}{X}, & \mathscr{D}_{2} I_{8,0} & =6 Y
\end{array}
$$


Looking at these equations, we see that we can solve the $8^{\text {th }}$ order invariant $I_{8,0}$ in terms of $X, Y$ and their invariant derivatives. An elementary induction yields:

Proposition 20.4. Within the branch $S \neq 0, W \equiv 0$, the algebra of differential invariants is generated by $X, Y$ and their invariant derivatives.

Moreover, $Y$ cannot be obtained from $X$ and its invariant derivatives.

Proof. Indeed, both derivatives $\mathscr{D}_{1} X=0$ and $\mathscr{D}_{2} X=5 X$ do not raise the jet order.

20.5. Commutators of invariant differentials. Besides taking invariant derivatives $\mathscr{D}_{1}$, $\mathscr{D}_{2}$, as in [33], there is another way to get syzygies among differential invariants: by means of the commutator $\left[\mathscr{D}_{1}, \mathscr{D}_{2}\right]$. For our group $\mathrm{SA}_{3}(\mathbb{R})$, Olver in [33] obtained the following formulas:

$$
\begin{aligned}
\mathscr{D}_{3}:=\left[\mathscr{D}_{1}, \mathscr{D}_{2}\right] & :=\mathscr{D}_{1} \circ \mathscr{D}_{2}-\mathscr{D}_{2} \circ \mathscr{D}_{1} \\
& =Z_{1} \mathscr{D}_{1}+Z_{2} \mathscr{D}_{2},
\end{aligned}
$$

with the two differential invariants:

$$
\begin{aligned}
& Z_{1}:=\sum_{1 \leqslant \sigma \leqslant 6}\left(\frac{\partial \xi_{\sigma}}{\partial x}(0,0,0) \boldsymbol{K}_{2}^{\sigma}-\frac{\partial \xi_{\sigma}}{\partial y}(0,0,0) \boldsymbol{K}_{1}^{\sigma}\right)=\boldsymbol{K}_{2}^{1}-\boldsymbol{K}_{1}^{3}, \\
& \boldsymbol{Z}_{2}:=\sum_{1 \leqslant \sigma \leqslant 6}\left(\frac{\partial \eta_{\sigma}}{\partial x}(0,0,0) \boldsymbol{K}_{2}^{\sigma}-\frac{\partial \eta_{\sigma}}{\partial y}(0,0,0) \boldsymbol{K}_{1}^{\sigma}\right)=\boldsymbol{K}_{2}^{5}-\boldsymbol{K}_{1}^{2} .
\end{aligned}
$$

Within the branch $S \neq 0, W \neq 0$, we have:

$$
\begin{aligned}
& Z_{1}=0-1=-1, \\
& Z_{2}=\frac{4}{3} W-W=\frac{1}{3} W .
\end{aligned}
$$

Hence $\left[\mathscr{D}_{1}, \mathscr{D}_{2}\right]=-\mathscr{D}_{1}+\frac{1}{3} W \mathscr{D}_{2}$, an operator which can raise the order by at most 1. The commutator does not generate anything other than the invariant derivations $\mathscr{D}_{1}$, $\mathscr{D}_{2}$ would do. One can double-check this formula by calculating $\left[\mathscr{D}_{1}, \mathscr{D}_{2}\right] W=\frac{4}{3} W^{2}=$ $-\mathscr{D}_{1} W+\frac{1}{3} W \mathscr{D}_{2} W$.

Note that when $\mathscr{D}_{2} M \neq 0$, from

$$
\mathscr{D}_{3} M=-\mathscr{D}_{1} M+\frac{1}{3} W \mathscr{D}_{2} M,
$$

the invariant $W$ is solved in terms of $M$ and its invariant differentials, so are all the other differential invariants; indeed, we check that $\mathscr{D}_{2} M \not \equiv 0$ in general, with numerator having 107 differential monomials.

Within the branch $S \neq 0, W \equiv 0$, we have:

$$
\begin{aligned}
& Z_{1}=0-1=-1, \\
& Z_{2}=0-0=0 .
\end{aligned}
$$

Hence $\left[\mathscr{D}_{1}, \mathscr{D}_{2}\right]=-\mathscr{D}_{1}$, an operator which can raise the order by at most 1 . The commutator does not generate anything other than the invariant derivations $\mathscr{D}_{1}, \mathscr{D}_{2}$ would do. One can double-check this formula by calculating $\left[\mathscr{D}_{1}, \mathscr{D}_{2}\right] X=0=-\mathscr{D}_{1} X$. 


\section{Explicit Invariant Differentiation Operators $\mathscr{D}_{1}$ and $\mathscr{D}_{2}$}

In order to double-check the overall theoretical coherency of our recurrence formulas satisfied by infinitely many differential invariants, let us raise

Question 21.1. How to make explicit the two invariant differentiation operators $\mathscr{D}_{1}$ and $\mathscr{D}_{2}$ ?

To fix ideas, we will content ourselves to examine the branch $W \neq 0$. One strategy would be to follow the general theory presented in the previous sections, but we already saw in Section 11 that it seems impossible to directly compute in terms of a cross-section, which we were unable to make explicit.

Therefore, we will trace another route. At first, applying the power series method of Sections $\mathbf{1 7}$ and $\mathbf{1 8}$, we compute explicitly the differential invariants $W \equiv I_{3,1}, M \equiv I_{5,0}$ (already shown) and also $I_{6,0}, I_{5,1}$. The numerator of $I_{6,0}$ has 225 monomials, that of $I_{5,1}$ (only) 69.

In advance, on a computer, we declare some dependent parabolic jets:

$$
u_{0,2}:=\frac{u_{1,1}^{2}}{u_{2,0}}, \quad u_{1,2}:=\frac{2 u_{2,0} u_{1,1} u_{2,1}-u_{1,1}^{2} u_{3,0}}{u_{2,0}^{2}},
$$

and so on, up to $u_{5,2}$ (those which will be useful for $\mathrm{D}_{y}$ below), and we declare the two total differentiation (not invariant) operators:

$$
\begin{aligned}
\mathrm{D}_{x}:= & \frac{\partial}{\partial x} \\
& +u_{1,0} \frac{\partial}{\partial u} \\
& +u_{2,0} \frac{\partial}{\partial u_{1,0}}+u_{1,1} \frac{\partial}{\partial u_{0,1}} \\
& +u_{3,0} \frac{\partial}{\partial u_{2,0}}+u_{2,1} \frac{\partial}{\partial u_{1,1}} \\
& +u_{4,0} \frac{\partial}{\partial u_{3,0}}+u_{3,1} \frac{\partial}{\partial u_{2,1}} \\
& +u_{5,0} \frac{\partial}{\partial u_{4,0}}+u_{4,1} \frac{\partial}{\partial u_{3,1}} \\
& +u_{6,0} \frac{\partial}{\partial u_{5,0}}+u_{5,1} \frac{\partial}{\partial u_{4,1}} \\
& +u_{7,0} \frac{\partial}{\partial u_{6,0}}+u_{6,1} \frac{\partial}{\partial u_{5,1}}
\end{aligned}
$$

and

$$
\begin{aligned}
\mathrm{D}_{y}:= & \frac{\partial}{\partial y} \\
& +u_{0,1} \frac{\partial}{\partial u} \\
& +u_{1,1} \frac{\partial}{\partial u_{1,0}}+u_{0,2} \frac{\partial}{\partial u_{0,1}} \\
& +u_{2,1} \frac{\partial}{\partial u_{2,0}}+u_{1,2} \frac{\partial}{\partial u_{1,1}} \\
& +u_{3,1} \frac{\partial}{\partial u_{3,0}}+u_{2,2} \frac{\partial}{\partial u_{2,1}}
\end{aligned}
$$$$
+u_{4,1} \frac{\partial}{\partial u_{4,0}}+u_{3,2} \frac{\partial}{\partial u_{3,1}}
$$$$
+u_{5,1} \frac{\partial}{\partial u_{5,0}}+u_{4,2} \frac{\partial}{\partial u_{4,1}}
$$$$
+u_{6,1} \frac{\partial}{\partial u_{6,0}}+u_{5,2} \frac{\partial}{\partial u_{5,1}} \text {. }
$$

According to the general theory, there are coefficients $\alpha, \beta, \gamma, \delta$ so that:

$$
\mathscr{D}_{1}=\alpha \mathrm{D}_{x}+\beta \mathrm{D}_{y} \quad \text { and } \quad \mathscr{D}_{2}=\gamma \mathrm{D}_{x}+\delta \mathrm{D}_{y} .
$$

Consequently, two appropriate pairs of recurrence relations seen in Section 20 read as two linear systems satisfied by the two pairs $\{\alpha, \beta\}$ and $\{\gamma, \delta\}$ :

$$
\left[\begin{array}{l}
\alpha \mathrm{D}_{x} W+\beta \mathrm{D}_{y} W=-\frac{2}{3} W^{2}, \\
\alpha \mathrm{D}_{x} M+\beta \mathrm{D}_{y} M=I_{6,0}-14 W M+\frac{10}{3} W I_{5,1},
\end{array}\right.
$$

and:

$$
\left[\begin{array}{l}
\gamma \mathrm{D}_{x} W+\delta \mathrm{D}_{y} W=2 W \\
\gamma \mathrm{D}_{x} M+\delta \mathrm{D}_{y} M=I_{5,1}-M+\frac{80}{9} W^{3}
\end{array}\right.
$$


Contrary to what could be expected/hoped, the common determinant:

$\Delta:=\left|\begin{array}{cc}\mathrm{D}_{x} W & \mathrm{D}_{y} W \\ \mathrm{D}_{x} \boldsymbol{M} & \mathrm{D}_{y} \boldsymbol{M}\end{array}\right|=-\frac{1}{54} \frac{\text { complicated numerator }}{u_{2,0}^{8}\left(u_{2,0} u_{2,1}-u_{1,1} u_{3,0}\right)^{8 / 3}\left(u_{1,1} u_{2,0} u_{4,0}-u_{2,0}^{2} u_{3,1}+2 u_{2,0} u_{2,1} u_{3,0}-2 u_{1,1} u_{3,0}^{2}\right)}$,

is not simple, as its numerator is a non-factorizable homogeneous polynomial of degree 15 in the jet variables up to order 6 having $\mathbf{4 3 1}$ monomials.

For some time, we were afraid that this unpleasant numerator indicated there existed a mistake in our recurrence formulas.

But if one really applies the Cramér formulas to the first linear system:

$\alpha:=\frac{\left|\begin{array}{cc}-\frac{2}{3} W^{2} & \mathrm{D}_{y} W \\ I_{6,0}-14 W M+\frac{10}{3} W I_{5,1} & \mathrm{D}_{y} M\end{array}\right|}{\left|\begin{array}{ll}\mathrm{D}_{x} W & \mathrm{D}_{y} W \\ \mathrm{D}_{x} \boldsymbol{M} & \mathrm{D}_{y} \boldsymbol{M}\end{array}\right|}, \quad \beta:=\frac{\left|\begin{array}{cc}\mathrm{D}_{x} W & -\frac{2}{3} W^{2} \\ \mathrm{D}_{x} \boldsymbol{M} & I_{6,0}-14 W M+\frac{10}{3} W I_{5,1}\end{array}\right|}{\left|\begin{array}{cc}\mathrm{D}_{x} W & \mathrm{D}_{y} W \\ \mathrm{D}_{x} \boldsymbol{M} & \mathrm{D}_{y} \boldsymbol{M}\end{array}\right|}$,

one realizes that this large complicated numerator is in fact an extraneous factor, namely it cancels as it appears both in numerator and in denominator places for both $\alpha$ and $\beta$.

After clearing this factor and cleaning, we receive:

$$
\begin{aligned}
\alpha:= & \frac{1}{6} \frac{1}{u_{2,0}\left(u_{2,0} u_{2,1}-u_{1,1} u_{3,0}\right)^{2 / 3}\left(u_{1,1} u_{2,0} u_{4,0}-u_{2,0}^{2} u_{3,1}+2 u_{2,0} u_{2,1} u_{3,0}-2 u_{1,1} u_{3,0}^{2}\right)}\{ \\
& \left\{12 u_{3,0} u_{2,1}^{2} u_{2,0}^{2}-6 u_{3,1} u_{2,1} u_{2,0}^{3}-44 u_{3,0}^{2} u_{1,1} u_{2,1} u_{2,0}+16 u_{3,0} u_{3,1} u_{1,1} u_{2,0}^{2}+15 u_{4,0} u_{1,1} u_{2,1} u_{2,0}^{2}\right. \\
& \left.-3 u_{1,1} u_{4,1} u_{2,0}^{3}+32 u_{3,0}^{3} u_{1,1}^{2}-25 u_{4,0} u_{1,1}^{2} u_{3,0} u_{2,0}+3 u_{5,0} u_{1,1}^{2} u_{2,0}^{2}\right\},
\end{aligned}
$$

and:

$$
\begin{aligned}
\beta:=\frac{1}{6} & \frac{1}{\left(u_{2,0} u_{2,1}-u_{1,1} u_{3,0}\right)^{2 / 3}\left(u_{1,1} u_{2,0} u_{4,0}-u_{2,0}^{2} u_{3,1}+2 u_{2,0} u_{2,1} u_{3,0}-2 u_{1,1} u_{3,0}^{2}\right)}\{ \\
& \left\{20 u_{2,0} u_{2,1} u_{3,0}^{2}-10 u_{3,0} u_{2,0}^{2} u_{3,1}-9 u_{2,0}^{2} u_{2,1} u_{4,0}+3 u_{4,1} u_{2,0}^{3}-20 u_{1,1} u_{3,0}^{3}\right. \\
& \left.+19 u_{3,0} u_{1,1} u_{2,0} u_{4,0}-3 u_{1,1} u_{2,0}^{2} u_{5,0}\right\} .
\end{aligned}
$$

The same extraneous factor from $\Delta$ also disappears from Cramér's formulas in the second linear system, and we receive rather neat and simpler expressions for:

$$
\gamma:=-\frac{u_{2,0} u_{1,1}}{u_{2,0} u_{2,1}-u_{1,1} u_{3,0}}
$$

and for:

$$
\delta:=\frac{u_{2,0}^{2}}{u_{2,0} u_{2,1}-u_{1,1} u_{3,0}} .
$$

A computation of the determinant:

$$
\left|\begin{array}{cc}
\alpha & \beta \\
\gamma & \delta
\end{array}\right|=\frac{u_{2,0}}{\left(u_{2,0} u_{2,1}-u_{1,1} u_{3,0}\right)^{2 / 3}} .
$$

confirms a property stated by the general theory:

$$
\operatorname{Span}\left\{\mathscr{D}_{1}, \mathscr{D}_{2}\right\}=\operatorname{Span}\left\{\mathrm{D}_{x}, \mathrm{D}_{y}\right\} \text {. }
$$


Lastly, with these explicit formulas for $\mathscr{D}_{1}$ and $\mathscr{D}_{2}$, we verify on a computer, after computing independently also, say, $I_{7,0}, I_{6,1}$ (and so on), that the next few recurrence formulas hold identically.

In conclusion, this confirms explicit coherency of all our formulas.

\section{Relation with the classification of developable surfaces: $\mathrm{SA}_{3}(\mathbb{R})$-invariant PDEs for cylinders and cones}

A surface in $\mathbb{R}^{3}$ is called a ruled surface if it can be parametrized by a family of lines (rulers):

$$
\vec{x}(t, v)=\vec{\alpha}(t)+v \vec{w}(t) \quad(t \in(0,1), v \in \mathbb{R}),
$$

where $\vec{\alpha}(t), \vec{w}(t)$ are $C^{1}$-smooth. It is called developable if

$$
\left(\vec{w}, \overrightarrow{w^{\prime}}, \overrightarrow{\alpha^{\prime}}\right) \equiv 0
$$

Properties of ruled surfaces and developable surfaces can be found in [14, 7]. For instance, a $C^{2}$-smooth surface is developable if and only if its Gaussian curvature is identically 0 . In this section, we will assume as before that geometric objects are analytic.

Parabolic surfaces are developable since their Gaussian curvature is constantly zero. A developable surface is called:

- cylindrical if all rules are parallel;

- conical if all rulers pass through the same point;

- tangential if all rulers are tangent to a certain curve in $\mathbb{R}^{3}$.

Near a $C^{2}$-smooth point, a developable surface is locally cylindrical or conical or tangential [7, p. 197].

Being locally cylindrical (or conical, or tangential) is clearly a $\mathrm{SA}_{3}(\mathbb{R})$-invariant local property. It is expected that such properties can be characterized by some differential $\mathrm{SA}_{3}(\mathbb{R})$-invariants. Indeed

Theorem 22.1. A parabolic surface is

- a cylinder if and only if $\boldsymbol{S} \equiv 0$,

- a cone if and only if $\boldsymbol{S} \neq 0$ and $W \equiv 0$,

- a tangential surface if and only if $S \neq 0$ and $W \neq 0$.

In [14, p. 295], the degenerate branches of cylinders and cones are characterized by the vanishing of some coefficients in the Cartan's structure equations.

Proof. It suffices to prove that at any smooth point of

(1) any cylinder, $S \equiv 0$,

(2) any cone, $S \neq 0$ and $W \equiv 0$,

(3) any tangential surface, $S \neq 0$ and $W \neq 0$.

(1) Any cylinder passing by a point $\vec{p} \in \mathbb{R}^{3}$, after some $\mathrm{SA}_{3}(\mathbb{R})$ action, can be viewed as $\mathbb{R}_{y}$ times a curve in $\mathbb{R}_{x, u}^{2}$, while $\vec{p}$ is mapped to the origin with the tangent plane $\mathbb{R}_{x, y}^{2}$. The cylinder is then a graph

$$
u=F(x)=\frac{F_{2,0}}{2} x^{2}+\frac{F_{3,0}}{6} x^{3}+\cdots .
$$

We calculate $S=\frac{F_{x x} F_{x x y}-F_{x y} F_{x x x}}{F_{x x}^{2}}=0$ at the origin. Since $\vec{p}$ is arbitrarily chosen, $S \equiv 0$. 


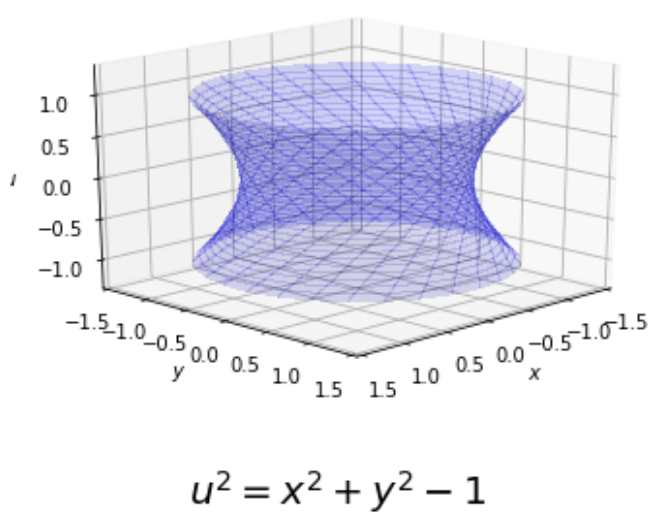

Figure 1. A nondevelopable ruled surface

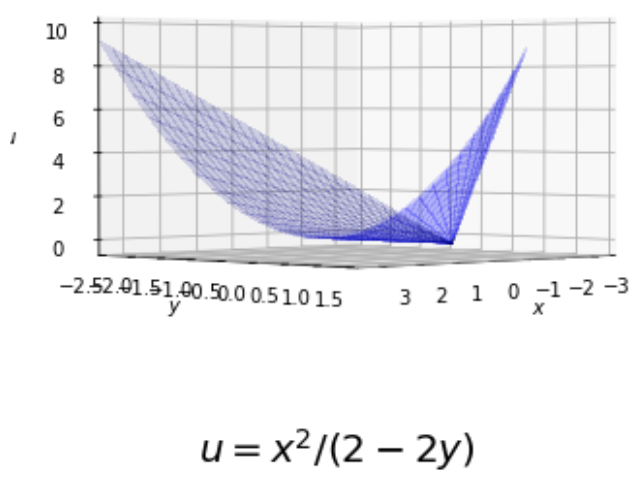

FIGURE 3. A conical surface

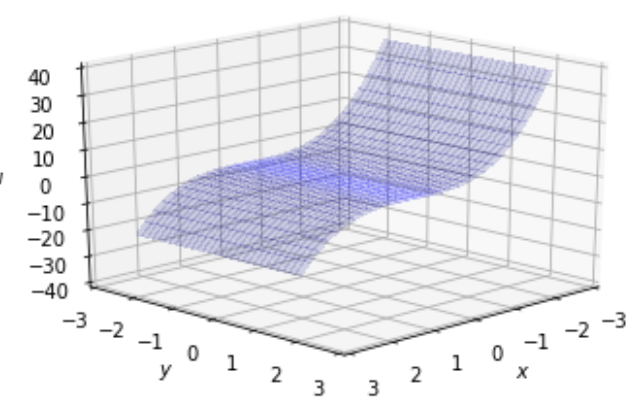

$$
u=x^{2}-x^{3}
$$

FIGURE 2. A cylindrical surface

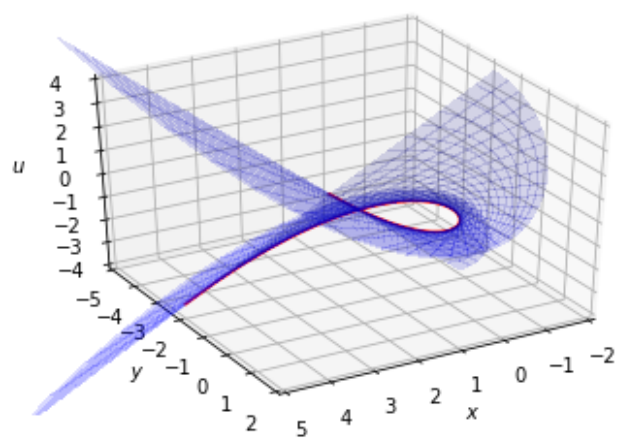

tangents of $\left(t^{2} / 2, t, t^{3} / 6\right)$

FIGURE 4. A tangential surface

(2) A cone can be parameterized by

$$
\vec{x}(t, v)=(1-v) \vec{\alpha}(t)+v \vec{p},
$$

where $v<1, t \in(-1,1), \vec{\alpha}(t)$ parametrizes a smooth directrix and $A:=\vec{p}$ is the apex.

For any smooth marked point $B$ on the cone, we apply the following three steps of $\mathrm{SA}_{3}(\mathbb{R})$-actions.

First, we translate $B$ to the origin.

Second, we fix the origin and rotate the cone so that its tangent plane at $B$ is spanned by $\vec{e}_{x}:=(1,0,0)$ and $\vec{e}_{y}:=(0,1,0)$, while the generatrix $\overrightarrow{B A}$ is parallel to $\overrightarrow{e_{y}}$.

Finally, we apply a dilation of the type $x^{\prime}=\lambda x, y^{\prime}=\lambda^{-1} y, u^{\prime}=u$ to make sure $|B A|=1$, i.e. $A=(0,1,0)$.

The new cone has the apex $(0,1,0)$ and the marked point $(0,0,0)$. By intersecting this new cone with $\mathbb{R}_{x, u}^{2}=\{y=0\}$, we get another directrix $(t, 0, c(t))$ passing the origin. 
Thus, our cone is $\mathrm{SA}_{3}(\mathbb{R})$-equivalent to

$$
\begin{aligned}
& x(t, v)=(1-v) t, \\
& y(t, v)=v, \\
& u(t, v)=(1-v) c(t),
\end{aligned}
$$

where $c(t)$ is smooth (analytic) and $c(0)=c^{\prime}(0)=0$. Since the marked point $B$ is arbitrarily chosen, it suffices to check that for the new cone above, the invariant $S \neq 0$ and $W=0$ at the origin.

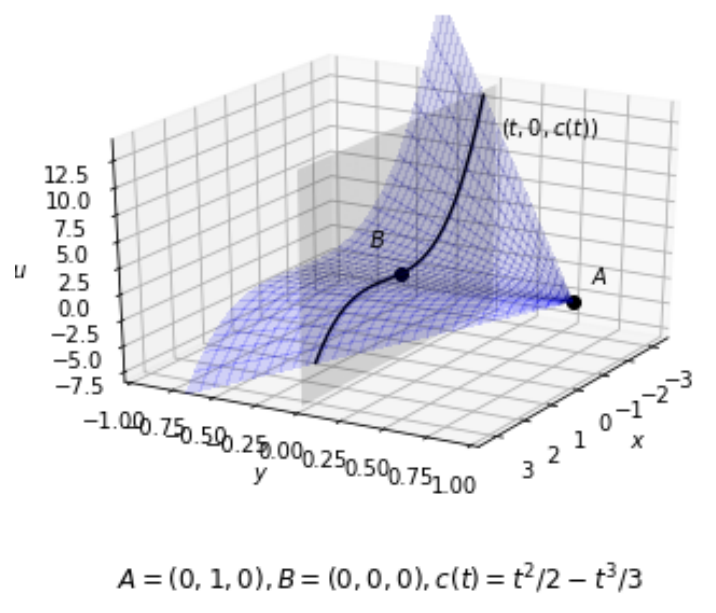

FIGURE 5. $\mathrm{A} \mathrm{SA}_{3}(\mathbb{R})$-normalized cone

Suppose such a conical surface is a graph of $u=F(x, y)$ around the origin. Assuming $c(t), F(x, y)$ analytic, expand

$$
\begin{aligned}
c(t) & =\frac{c_{2}}{2} t^{2}+\frac{c_{3}}{6} t^{3}+\cdots, \\
F(x, y) & =F_{1,0} x+F_{0,1} y+\frac{F_{2,0}}{2} x^{2}+F_{1,1} x y+\frac{F_{0,2}}{2} y^{2}+\cdots .
\end{aligned}
$$

In the fundamental identity holding for $t, v$ near 0 :

$$
u(t, v) \equiv F(x(t, v), y(t, v)),
$$

the Taylor coefficients of all monomials $t^{j} v^{k}$ should be the same. Identifying and solving, we get

$$
\begin{aligned}
& F_{1,0}=0, \quad F_{0,1}=0, \\
& F_{2,0}=c_{2}, \quad F_{1,1}=0, \quad F_{0,2}=0, \\
& F_{3,0}=c_{3}, \quad F_{2,1}=c_{2}, \quad F_{1,2}=0, \quad F_{0,3}=0, \\
& F_{4,0}=c_{4}, \quad F_{3,1}=2 c_{3}, \quad F_{2,2}=2 c_{2}, \quad F_{1,3}=0, \quad F_{0,4}=0 .
\end{aligned}
$$

We may then compute

$$
\begin{aligned}
S & :=\frac{F_{2,0} F_{2,1}-F_{1,1} F_{3,0}}{F_{2,0}^{2}} \\
W & :=\frac{2 F_{1,1} F_{3,0}^{2}+F_{2,0}^{2} F_{3,1}-2 F_{2,0} F_{2,1} F_{3,0}-F_{2,0} F_{1,1} F_{4,0}}{F_{2,0}^{2}\left(F_{2,0} F_{2,1}-F_{1,1} F_{3,0}\right)^{2 / 3}}
\end{aligned}
$$


to get $S=1$ and $W=0$.

Remark. In the branch $W \equiv 0$, there are 2 generators of $\mathrm{SA}_{3}(\mathbb{R})$-invariants: $X$ of order 5 and $Y$ of order 7 . Indeed at the origin of the normalized cone

$$
X=\frac{40 c_{3}^{3}-45 c_{2} c_{3} c_{4}+9 c_{2}^{2} c_{5}}{9 c_{2}^{4}}
$$

is the Monge invariant. The model $u=\frac{x^{2}}{2(1-y)}$ is a cone with apex $(0,1,0)$ and directrix $\left(t, 0, t^{2} / 2\right)$. One can verify that $W=0$ and $X=0$ at the origin.

(3) A tangential surface can be parametrized by

$$
\vec{x}(t, v)=\alpha(t)+v \alpha^{\prime}(t)
$$

where $v \in \mathbb{R}, t \in(-1,1)$ and $\vec{\alpha}(t)$ parametrizes a smooth (analytic) directrix.

For any smooth point $B=\vec{x}\left(t_{0}, v_{0}\right)$ on the surface, let $A=\vec{x}\left(t_{0}, 0\right)$ be the corresponding point on the directrix. There is a $\mathrm{SA}_{3}(\mathbb{R})$-action sending $A$ to $(0,-1,0)$ and $B$ to $(0,0,0)$. The original surface is sent to tangents of a curve passing by $(0,-1,0)$ with tangent direction $\overrightarrow{e_{y}}:=(0,1,0)$. The curve can be locally reparametrized as $(a(t),-1+t, c(t))$. Thus our tangential surface is $\mathrm{SA}_{3}(\mathbb{R})$-equivalent to

$$
\begin{aligned}
& x(t, v)=a(t)+v a^{\prime}(t), \\
& y(t, v)=-1+t+v, \\
& u(t, v)=c(t)+v c^{\prime}(t),
\end{aligned}
$$

where $v \in \mathbb{R}, t \in(-1,1), a(t)$ and $c(t)$ are analytic with $a(0)=c(0)=a^{\prime}(0)=c^{\prime}(0)=0$. Note that $\vec{x}(0,1)=(0,0,0)$ is the marked point $B$.

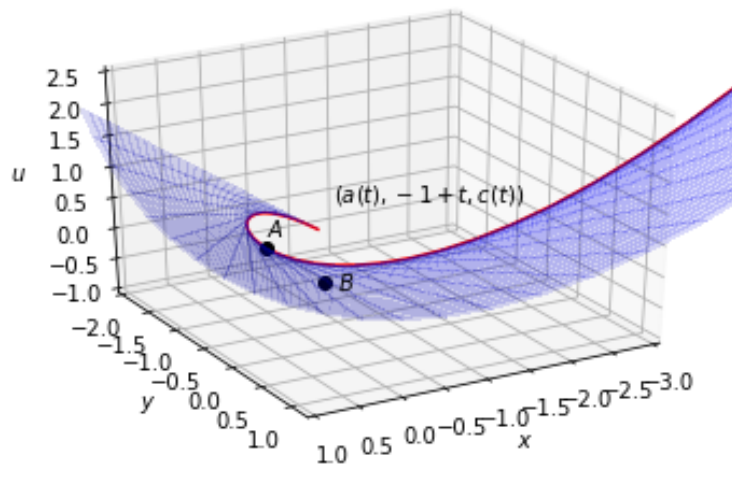

$$
A=(0,-1,0), B=(0,0,0), a(t)=-t^{2} / 2, c(t)=t^{3} / 6
$$

FIGURE 6. $\mathrm{A} \mathrm{SA}_{3}(\mathbb{R})$-normalized tangential surface

We may rotate the $(x, u)$-space while fixing the $y$-axis to make sure the surface is not vertical at the origin. Then the surface is graphed as $u=F(x, y)$. Assuming $a(t), c(t)$, 
$F(x, y)$ analytic in $t$, we have

$$
\begin{aligned}
a(t) & =\frac{a_{2}}{2} t^{2}+\frac{a_{3}}{6} t^{3}+\cdots, \\
c(t) & =\frac{c_{2}}{2} t^{2}+\frac{c_{3}}{6} t^{3}+\cdots, \\
F(x, y) & =F_{1,0} x+F_{0,1} y+\frac{F_{2,0}}{2} x^{2}+F_{1,1} x y+\frac{F_{0,2}}{2} y^{2}+\cdots .
\end{aligned}
$$

By expanding the fundamental identity holding for $(t, v)$ near $(0,1)$

$$
u(t, v) \equiv F(x(t, v), y(t, v)),
$$

by identifying Taylor coefficients of monomials $t^{j}(v-1)^{k}$, and by solving, we obtain

$$
\begin{gathered}
F_{1,0}=\frac{c_{2}}{a_{2}}, \quad F_{0,1}=0, \\
F_{2,0}=\frac{-a_{3} c_{2}+a_{2} c_{3}}{a_{2}^{3}}, \quad F_{1,1}=0, \quad F_{0,2}=0, \\
F_{3,0}=\frac{-a_{2} a_{3} c_{2}+3 a_{3}^{2} c_{2}-a_{2} a_{4} c_{2}+a_{2}^{2} c_{3}-3 a_{2} a_{3} c_{3}+a_{2}^{2} c_{4}}{a_{2}^{5}}, \\
F_{2,1}=\frac{a_{3} c_{2}-a_{2} c_{3}}{a_{2}^{3}}, \quad F_{1,2}=0, \quad F_{0,3}=0, \\
F_{4,0}=\frac{1}{a_{2}^{7}}\left(-3 a_{2}^{2} a_{3} c_{2}+10 a_{2} a_{3}^{2} c_{2}-15 a_{3}^{3} c_{2}-3 a_{2}^{2} a_{4} c_{2}+10 a_{2} a_{3} a_{4} c_{2}+a_{2}^{2} a_{5} c_{2}+3 a_{2}^{3} c_{3}\right. \\
\left.-10 a_{2}^{2} a_{3} c_{3}+15 a_{2} a_{3}^{2} c_{3}-4 a_{2}^{2} a_{4} c_{3}+3 a_{2}^{3} c_{4}-6 a_{2}^{2} a_{3} c_{4}+a_{2}^{3} c_{5}\right), \\
F_{3,1}=\frac{1}{a_{2}^{5}}\left(3 a_{2} a_{3} c_{2}-6 a_{3}^{2} c_{2}+2 a_{2} a_{4} c_{2}-3 a_{2}^{2} c_{3}+6 a_{2} a_{3} c_{4}+a_{2}^{3} c_{5}\right), \\
F_{2,2}=\frac{2\left(-a_{3} c_{2}+a_{2} c_{3}\right)}{a_{2}^{3}}, \quad F_{1,3}=0, \quad F_{0,4}=0 .
\end{gathered}
$$

A computation conducts to the compact expression

$$
W=\left(a_{3} c_{2}-a_{2} c_{3}\right)^{-1 / 3}
$$

On the other hand, observe that for the curve $\vec{\alpha}(t)=(a(t),-1+t, c(t))$, the torsion is

$$
\tau(t)=\frac{1+a^{\prime}(t)^{2}+c^{\prime}(t)^{2}}{\left(a^{\prime \prime}(t)^{2}+c^{\prime \prime}(t)^{2}\right)^{2}}\left(c^{\prime \prime}(t) a^{\prime \prime \prime}(t)-a^{\prime \prime}(t) c^{\prime \prime \prime}(t)\right) .
$$

We shall exclude the case $\tau(t) \equiv 0$, since in this degenerate case, the curve is locally planar, whence the tangential surface is locally flat, contradicting our assumption $F_{x x} \neq 0$. If $t=0$ is an isolated zero of $\tau(t)$, then the tangential surface has a cuspidal edge along $\vec{x}(0, v)[5]$, contradicting our overall assumption that the surface is smooth (and analytic) at $\vec{x}(0,1)$. Thus $\tau(0) \neq 0$ necessarily, and this guarantees that $c_{2} a_{3}-c_{3} a_{2} \neq 0$. In conclusion, $W \neq 0$. 


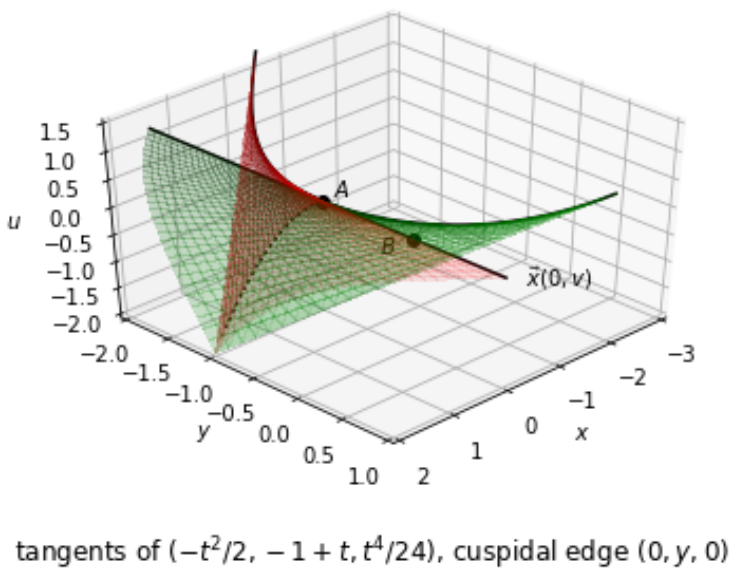

FIGURE 7. Tangential surface of a curve with an isolated zero torsion point at $A=(0,-1,0)$.

\section{Special Affinely Homogeneous Models}

The complete classification of $A_{3}(\mathbb{R})$-homogeneous surfaces $S^{2} \subset \mathbb{R}^{3}$ was terminated by Doubrov-Komrakov-Rabinovich [8], who solved a long-standing open problem. Also, Abdalla-Dillen-Vrancken [1] finished the delicate classification of affinely homogeneous surfaces in $\mathbb{R}^{3}$ having vanishing Pick invariant. This full classification was done again later by Eastwood-Ezhov [9], who employed the power series method, without considering algebras of differential invariants. In the literature, we did not find an answer to the simple

Question 23.1. What are the special affinely homogeneous surfaces $S^{2} \subset \mathbb{R}^{3}$ ?

Because any differential invariant $I$ satisfies, according to Sections 4 and 5 .

$$
I\left(g \cdot z^{(n)}\right)=I\left(z^{(n)}\right),
$$

when the horizontal group action, namely the projection of $g \cdot z^{(n)}$ :

$$
(x, \mathrm{u}(x)) \longmapsto(\varphi(g, x, \mathrm{u}(x)), \psi(g, x, \mathrm{u}(x))),
$$

is transitive, this forces $I$ to be constant.

Observation 23.2. All differential invariants are constant for geometric objects that are homogeneous.

Consequently, all invariant derivatives of any order $\geqslant 1$ are trivially zero:

$$
\mathscr{D}_{1}^{j_{1}} \cdots \mathscr{D}_{p}^{j_{p}} \boldsymbol{I} \equiv 0 \text {. }
$$

This yields interesting simplications in the recurrence relations of Theorem 14.6, that are better rewritten as:

$$
I_{K, j}^{\alpha}=\underline{\mathscr{D}_{j} I_{K}^{\alpha}}-\sum_{1 \leqslant \sigma \leqslant r} \varphi_{\sigma K}^{\alpha}\left(I^{(K)}\right) \cdot K_{j}^{\sigma}\left(I^{\left(n_{\mathrm{G}}+1\right)}\right) .
$$

Generally, the power series of the graphing functions $u^{\alpha}=F^{\alpha}\left(x^{1}, \ldots, x^{p}\right), \alpha=$ $1, \ldots, q$, can be fully determined from these recurrence relations, when the group action is transitive. We now illustrate this general idea in our elementary context. 


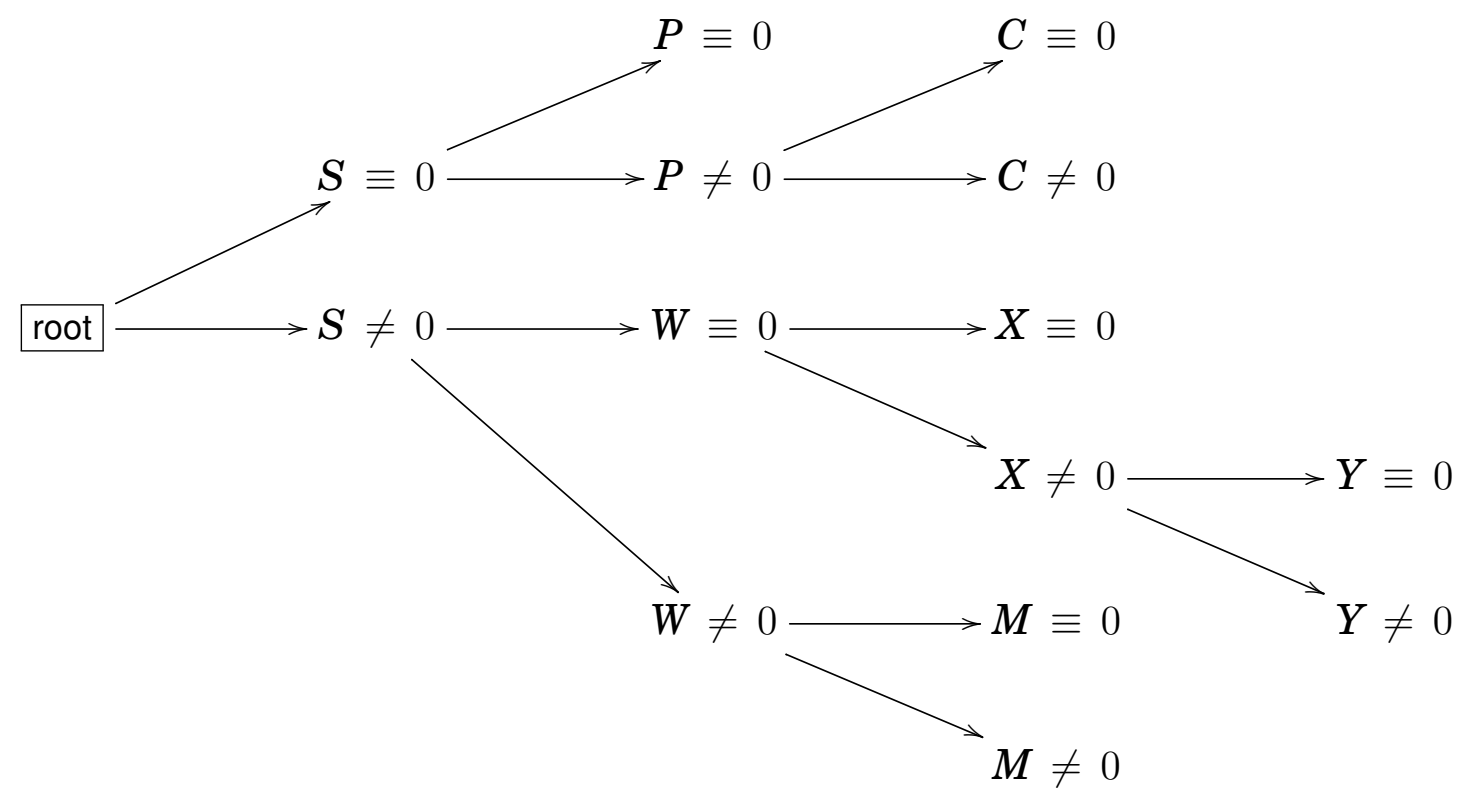

Coming back to the complete branching diagram of Section 2 copied above, we remember that the branch $S \equiv 0$ corresponds to cylindrical surfaces, namely surfaces that are the product of a curve $C^{1} \subset \mathbb{R}_{x, u}$ with $\mathbb{R}_{y}^{1}$. We also observe that $S A_{3}(\mathbb{R})$-equivalence classes of such cylindrical surfaces are in one-to-one correspondence with $\mathrm{A}_{2}(\mathbb{R})$-equivalence classes of the corresponding curves, because one can always use appropriate dilations along the dumb axes $\mathbb{R}_{y}^{1}$ to adjust preservation of the volume, so that the volume-preserving condition in $\mathbb{R}^{3}$ is not transmitted as an aera-preserving condition in $\mathbb{R}^{2}$.

Thus, let us discuss how $A_{2}(\mathbb{R})$-homogeneous curves can be determined by employing the recurrence relations for differential invariants shown in Section 16.

- Clearly, the relative invariant $I_{2}:=F_{x x}$ vanishes identically if and only if the curve is affinely equivalent to the straight line $\{u=0\}$.

- On the branch $I_{2} \neq 0$, there is the relative differential invariant $I_{4}:=\frac{1}{3} \frac{-5 F_{x x x}^{2}+3 F_{x x} F_{x x x x}}{F_{x x}^{2}}$. Then Lemma 13.10 (1) already showed that $I_{4} \equiv 0$ if and only if the curve is $A_{2}(\mathbb{R})$ equivalent to the parabola $\left\{u=x^{2}\right\}$.

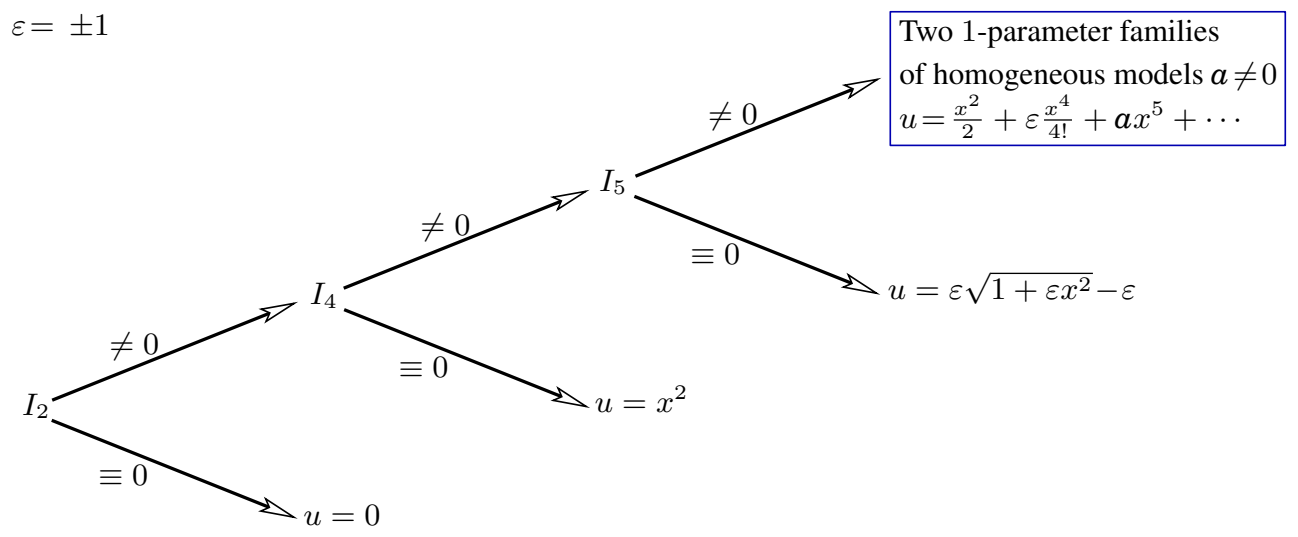


- On the branch $I_{2} \neq 0 \neq I_{4}$, with $\varepsilon:= \pm 1$ denoting the sign of $I_{4}$, there comes the first absolute (i.e. not relative) differential invariant:

$$
I_{5}:=\frac{1}{\sqrt{3}} \frac{9 F_{x x}^{2} F_{x x x x x}-45 F_{x x} F_{x x x} F_{x x x x}+40 F_{x x x}^{3}}{\left(\varepsilon\left[3 F_{x x} F_{x x x x}-5 F_{x x x}^{2}\right]\right)^{3 / 2}} .
$$

Then Lemma 13.10 (2) already showed that $\{u=F(x)\}$ is contained in a nondegenerate conic (hence not a parabola) if and only if $I_{5} \equiv 0$. One can verify that only two normalized equations occur:

$$
u=\varepsilon \sqrt{1+\varepsilon x^{2}}-\varepsilon,
$$

a circle for $\varepsilon=1$, and a hyperbola for $\varepsilon=-1$. Both are well known to be affinely homogeneous.

- Lastly, it remains to consider the branch $I_{5} \neq 0$. Then according to Observation 23.2, for homogeneity to hold, $I_{5} \equiv a \in \mathbb{R} \backslash\{0\}$ must necessarily be constant. Furthermore, the recurrence relations written at the end of Section 16 become:

$$
\begin{aligned}
& I_{6}=\mathscr{D}_{x}\left(I_{5}\right) \circ \pm \frac{3}{2} I_{5}^{2}+5, \quad= \pm \frac{3}{2} a^{2}+5, \\
& I_{7}=\mathscr{D}_{x}\left(I_{6}\right)_{\circ} \pm 2 I_{5} I_{6} \pm 7 I_{5}=3 a^{3} \pm 17 a \text {. }
\end{aligned}
$$

Beyond, we have for every $k \geqslant 5$ :

$$
I_{k+1}=\underline{\mathscr{D}_{x}\left(\boldsymbol{I}_{k}\right)} \circ-\sum_{1 \leqslant \kappa \leqslant 4} \operatorname{inv}\left(\Phi_{\kappa}^{k}\right) R^{\kappa}
$$

hence knowing the values of the $R^{\kappa}$, computing the values of $\operatorname{inv}\left(\Phi_{\kappa}^{k}\right)$, we realize that all $I_{k \geqslant 5}$ are uniquely determined polynomials in $a$. Thus, the power series reads:

23.3

$$
u=\frac{x^{2}}{2 !} \pm \frac{x^{4}}{4 !}+a \frac{x^{5}}{5 !}+\left(5 \pm \frac{3}{2} a^{2}\right) \frac{x^{6}}{6 !}+\left(3 a^{3} \pm 17 a\right) \frac{x^{7}}{7 !}+\cdots
$$

By employing the Cauchy majorant method, one could prove that this power series has a radius of convergence $>0$ for any parameter $a$, but this would be tedious.

Another more elementary and straightforward method is to test whether an affine infinitesimal transformation with 6 unknowns:

$$
L:=(\mathrm{A} x+\mathrm{B} u+\mathrm{C}) \frac{\partial}{\partial x}+(\mathrm{E} x+\mathrm{F} u+\mathrm{G}) \frac{\partial}{\partial u},
$$

is tangent to the above graph, and to realize by examining Taylor coefficients only up to order 5 , that one comes to the single solution - up to dilation - :

$$
L:=\left( \pm 1-\frac{1}{2} \boldsymbol{a} x-\frac{1}{3} u\right) \frac{\partial}{\partial x}+( \pm x-\boldsymbol{a} u) \frac{\partial}{\partial u} .
$$

Furthermore, one can verify that this vector field is indeed tangent to the graph 23.3 truncated to any order, and that the recurrence relations are equivalent to such a tangency condition.

Therefore, the homogeneous curve can simply be taken as the orbit of the origin $0 \in \mathbb{R}^{2}$ by the flow of the vector field $L$, and since $L$ is analytic with $L(0) \neq 0$, its flow and its local orbits are analytic too.

By construction, different values of the parameter $a \in \mathbb{R} \backslash\{0\}$ conduct to affinely inequivalent homogeneous curves. This is the main interest of the use of differential invariants.

Alternative classifications provide closed, explicit expressions for homogeneous graphs $\{u=F(x)\}$, but this then requires discussions (see e.g. [27]) about ranges of incoming parameters in order to determine in which precise (invariant) branches do sit the corresponding homogeneous models. We will not touch these aspects here. 

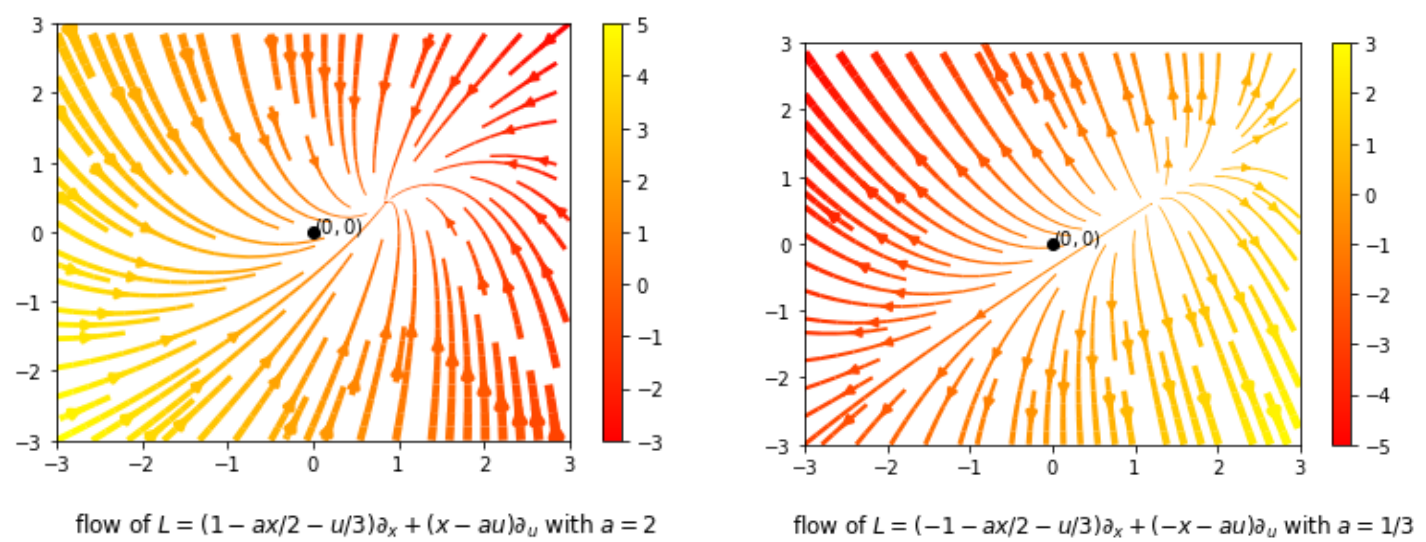

FIGURE 8. Flow of $L$

Now, we come to the non-cylindrical surfaces, those having $S \neq 0$. From Theorem 19.4 , we already know that when $W \equiv 0 \equiv X$, there is, up to $\mathrm{SA}_{3}(\mathbb{R})$, the single graph:

$$
u=\frac{1}{2} \frac{x^{2}}{1-y}
$$

which is equivalent to a smooth part of the standard straight cone $\left\{x_{1}^{2}+x_{2}^{2}-x_{3}^{2}=0\right\}$. Furthermore, one can verify that this model is special affinely homogeneous, with its algebra of infinitesimal transformations contained in $\mathfrak{s a}_{3}(\mathbb{R})$ being generated by:

$$
\begin{aligned}
& e_{1}:=-u \partial_{x}+x \partial_{y}, \\
& e_{2}:=(1-y) \partial_{x}+x \partial_{u}, \\
& e_{3}:=(1-y) \partial_{y}+u \partial_{u},
\end{aligned}
$$

with Lie structure

$$
\begin{aligned}
& {\left[e_{1}, e_{2}\right]=-e_{3},} \\
& {\left[e_{1}, e_{3}\right]=-e_{1},} \\
& {\left[e_{2}, e_{3}\right]=e_{2},}
\end{aligned}
$$

isomorphic to $\mathfrak{s l}_{2}(\mathbb{R})$.

Observation 23.4. Excepting the straight cone $\left\{u=\frac{1}{2} \frac{x^{2}}{1-y}\right\}$, there are no non-cylindrical special affinely homogeneous parabolic surfaces $S^{2} \subset \mathbb{R}^{3}$.

Proof. Coming back to the complete branching diagram copied above, it remains to examine the two branches $W \equiv 0 \neq X$ and $W \neq 0$, with no need of going to subbranches.

- When $W \equiv 0 \neq X$, by homogeneity we have $X=: a \in \mathbb{R} \backslash\{0\}$ constant, but one of the recurrence relations shown at the end of Subsection 20.3 immediately brings a contradiction:

$$
0=\mathscr{D}_{2} X=3 X
$$

- When $W \neq 0$, we also have $W=: b \in \mathbb{R} \backslash\{0\}$ constant, but one of the recurrence relations shown at the end of Subsection 20.1 again brings an immediate contradiction:

$$
0=\mathscr{D}_{2} W=2 W \text {. }
$$

\section{REFERENCES}

[1] Abdalla, B.; Dillen, F.; Vrancken, L.: Affine homogeneous surfaces in $\mathbb{R}^{3}$ with vanishing Pick invariant, Abh. Math. Sem. Univ. Hamburg 67 (1997), 105-115.

[2] Auffray, N.; Kolev, B.; Olive, M.: A minimal integrity basis for the elasticity tensor, Arch. Ration. Mech. Anal. 226 (2017), no. 1, 1-31. 
[3] Blaschke, W.: Affine Differentialgeometrie, Vorlesungen über Differentialgeometrie und geometrische Grundlagen von Einsteins Relativitätstheorie, II. Springer-Verlag, Berlin, 1923.

[4] Bluman, G.W.; Kumei, S.: Symmetries and differential equations, Applied Mathematical Sciences 81, Springer Verlag, New York, 1989, xiv+412 pp.

[5] Cleave, J.P.: The form of the tangent-developable at points of zero torsion on space curves, Math. Proc. Cambridge Philos. Soc. 88 (1980), no. 3, 403-407.

[6] Desmorat, R.; Kolev, B.; Olive, M.: Harmonic factorization and reconstruction of the elasticity tensor, J. Elasticity 132 (2018), no. 1, 67-101.

[7] Do Carmo, M. P.: Differential geometry of curves \& surfaces., Revised \& updated second edition. Dover Publications, Inc., Mineola, NY, 2016. xvi+510 pp.

[8] Doubrov, B.; Komrakov, B.; Rabinovich, M.: Homogeneous surfaces in the three-dimensional affine geometry, Geometry and topology of submanifolds, VIII (Brussels, 1995/Nordfjordeid, 1995), 168-178, World Sci. Publ., River Edge, NJ, 1996.

[9] Eastwood, M.; Ezhov, V.: On affine normal forms and a classification of homogeneous surfaces in affine threespace, Geom. Dedicata 77 (1999), no. 1, 11-69.

[10] Engel, F.; Lie, S.: Theorie der Transformationsgruppen. Erster Abschnitt. Unter Mitwirkung von Prof. Dr. Friedrich Engel, bearbeitet von Sophus Lie, Verlag und Druck von B.G. Teubner, Leipzig und Berlin, xii+638 pp. (1888). Reprinted by Chelsea Publishing Co., New York, N.Y. (1970).

[11] Fels, M.; Olver, P. J.: On relative invariants, Math. Ann. 308 (1997), no. 4, 701-732.

[12] Fels, M.; Olver, P.J.: Moving coframes. I. A practical algorithm, Acta Appl. Math. 51 (1998), no. 2, 161-213.

[13] Fels, M.; Olver, P.J.: Moving coframes. II. Regularization and theoretical foundations, Acta Appl. Math. 55 (1999), no. 2, 127-208.

[14] Guggenheimer, H.W.: Differential Geometry, Corrected reprint of the 1963 edition. Dover Books on Advanced Mathematics. Dover Publications, Inc., New York, 1977, x+378 pp.

[15] Halphen, G.H.: Sur l'équation différentielle des coniques, Bulletin de la Société mathématique, t. VII, 1878-1879, pp. 83-84.

[16] Hubert, E.; Olver, P.J.: Differential invariants of conformal and projective surfaces, SIGMA, Symmetry Integrability Geom. Methods Appl. 3 (2007), Paper 097, 15 pp.

[17] Isaev, A.: Spherical tube hypersurfaces, Lecture Notes in Mathematics, 2020, Springer, Heidelberg, 2011, $\mathrm{xii}+220 \mathrm{pp}$.

[18] Isaev, A.: Affine rigidity of Levi degenerate tube hypersurfaces, J. Differential Geom. 104 (2016), no. 1, 111-141.

[19] Isaev, A.: Zero CR-curvature equations for Levi degenerate hypersurfaces via Pocchiola's invariants, arxiv.org/abs/1809.03029/, Ann. Fac. Sci. Toulouse, to appear.

[20] Jensen, G.: Higher order contact of submanifolds of homogeneous spaces, Lecture Notes in Mathematics, Vol. 610. Springer-Verlag, Berlin-New York, 1977, xii+154 pp.

[21] Lie, S.: Klassifikation und Integration von gewöhnlichen Differentialgleichungen zwischen x, y, die eine Gruppe von Transformationen gestaten I-IV. In: Gesammelte Abhandlungen, Vol. 5, B.G. Teubner, Leipzig, 1924, pp. 240310; 362-427, 432-448.

[22] Lie, S. (Author); Merker, J. (Editor): Theory of Transformation Groups I. General Properties of Continuous Transformation Groups. A Contemporary Approach and Translation, Springer-Verlag, Berlin, Heidelberg, 2015, $\mathrm{xv}+643$ pp. arxiv.org/abs/1003.3202/

[23] Merker, J.: Characterization of the Newtonian free particle system in $m \geqslant 2$ dependent variables, Acta Applicandæ Mathematicæ 92 (2006), no. 2, 125-207.

[24] Merker, J.: Lie symmetries of partial differential equations and CR geometry, Journal of Mathematical Sciences (N.Y.), 154 (2008), 817-922.

[25] Merker, J.: Rationality in Differential Algebraic Geometry, Complex Geometry and Dynamics, The Abel Symposium 2013, Abel Symposia, Vol. 10, Fornæss, John Erik, Irgens, Marius, Wold, Erlend Fornæss (Eds.), pp. 157209, Springer-Verlag, Berlin, 2015.

[26] Merker, J.: Affine rigidity without integration, arxiv.org/abs/1903.00889/, 28 pages.

[27] Merker, J.; Nurowski, P.: Homogeneous CR and para-CR structures in dimensions 5 and 3, upcoming on arxiv.org/, 34 pages.

[28] Olive, M.: About Gordan's algorithm for binary forms, Found. Comput. Math. 17 (2017), no. 6, 1407-1466.

[29] Olver, P.J.: Applications of Lie groups to differential equations. Second edition. Graduate Texts in Mathematics, 107. Springer-Verlag, New York, 1993, xxviii+513 pp. 
[30] Olver, P.J.: Equivalence, Invariance and Symmetries. Cambridge, Cambridge University Press, 1995, xvi+525 pp.

[31] Olver, P.J.: Moving frames and singularities of prolonged group actions, Selecta Math. (N.S.) 6 (2000), no. 1, 41-77.

[32] Olver, P.J.: Joint invariant signatures, Found. Comput. Math. 1 (2001), no. 1, 3-67.

[33] Olver, P.J.: Differential invariants of surfaces, Differential Geom. Appl. 27 (2007), no. 2, 230-239.

[34] Olver, P.J.: Generating differential invariants, J. Math. Anal. Appl. 333 (2007), no. 1, 450-471.

[35] Olver, P.J.: Lectures on moving frames, in: Symmetries and Integrability of Difference Equations, D. Levi, P. Olver, Z. Thomova, and P. Winternitz, eds., London Math. Soc. Lecture Notes Series, vol. 381, Cambridge University Press, Cambridge, 2011, pp. 207-246.

[36] Olver, P.J.: Normal forms for submanifolds under group actions, Symmetries, differential equations and applications, 1-25. Springer Proc. Math. Stat. 266, Springer, Cham, 2018.

[37] Olver, P.J.; Pohjanpelto, J.: Moving frames for Lie pseudo-groups, Canad. J. Math. 60 (2008), no. 6, 1336-1386.

[38] Olver, P.J.; Pohjanpelto, J.: Differential invariant algebras of Lie pseudo-groups, Adv. Math. 222 (2009), no. 5, 1746-1792.

[39] Pocchiola, S.: Explicit absolute parallelism for 2-nondegenerate real hypersurfaces $M^{5} \subset \mathbb{C}^{3}$ of constant Levi rank 1, arxiv.org/abs/1312.6400/, 55 pages.

[40] Spivak, M.: A comprehensive introduction to differential geometry, Vol. III, Second Ed. Publish or Perish, Inc., Wilmington, Del., 1979. 